\title{
SERIAL INTRAVASCULAR ULTRASOUND ASSESSMENT OF CORONARY ATHEROSCLEROSIS PROGRESSION AND REMODELING
}

Marc Hartmann 
ISBN 978-90-713-8269-7

Copyright (C) 2008 Marc Hartmann, Enschede, The Netherlands All rights are reserved. No part of this publication may be reproduced, stored in a retrieval system or transmitted in any form or by any means, electronic, mechanical, photocopying, recording or otherwise, without the prior written permission of the author, or, when applicable, of the publishers of the scientific papers.

Printed by Gildeprint Drukkerijen, Enschede, The Netherlands

Cover: Marc Hartmann (“IVUS Wall”) 


\title{
SERIAL INTRAVASCULAR ULTRASOUND ASSESSMENT OF CORONARY ATHEROSCLEROSIS PROGRESSION AND REMODELING
}

\section{PROEFSCHRIFT}

\author{
ter verkrijging van \\ de graad van doctor aan de Universiteit Twente, \\ op gezag van de rector magnificus, \\ Prof. dr. W.H.M. Zijm, \\ volgens besluit van het College voor Promoties \\ in het openbaar te verdedigen \\ op donderdag 27 november 2008 om 13.15 uur
}

door

Marc Hartmann

geboren op 03 februari 1978

te Duisburg, Duitsland 
This dissertation has been approved by the promotor:

Prof. dr. Clemens von Birgelen 
dedicated to Nadine and Leni 


\section{Members of the committee}

Chairman

Prof. dr. G. van der Steenhoven University Twente, Enschede

Promotor

Prof. dr. C. von Birgelen University Twente, Enschede

Members

Prof. dr. A.G.J.M. van Leeuwen University Twente, Enschede

Prof. dr. M.J. IJzerman

University Twente, Enschede

Prof. dr. P.J. de Feyter

Erasmus Medical Center, Rotterdam

Prof. dr. V. Subramaniam

University Twente, Enschede

Prof. dr. R.J.G. Peters

Academic Medical Center, Amsterdam

Prof. dr. G.P. Vooijs

University Twente, Enschede

\section{Support}

Financial support by Medisch Spectrum $\Delta$ Twente, Stichting Hartcentrum Twente, Stichting Kwaliteitsverbetering Cardiologie Enschede, Abbott Vascular, Astra Zeneca, Biotronik, Boehringer Ingelheim, Bristol-Myers Squibb, Cordis Johnson \& Johnson Medical, Daiichi-Sankyo, Edwards Lifesciences, Glaxo Smith Kline, Medtronic, Norvatis, Schering-Plough, St. Jude Medical, Pfizer, and Volcano for the publication of this thesis are gratefully acknowledged. 
Contents 



\section{CONTENTS}

Chapter 1

General introduction

Chapter 2

29

Relation between progression and regression of atherosclerotic left main coronary artery disease and serum cholesterol levels as assessed with serial long-term ( $\geq 12$ months) follow-up intravascular ultrasound

von Birgelen C, Hartmann M, Mintz GS, et al.

Circulation 2003;108:2757-2762.

Chapter 3

Relationship between plaque progression and low-density lipoprotein cholesterol during aging as assessed with serial long-term ( $\geq 12$ months) follow-up intravascular ultrasound of the left main coronary artery

Hartmann M, von Birgelen C, Mintz GS, et al.

The American Journal of Cardiology 2006;98:1419-1423.

Chapter 4

Relation between lipoprotein(a) and fibrinogen and serial intravascular ultrasound plaque progression in left main coronary arteries

Hartmann M, von Birgelen C, Mintz GS, et al.

Journal of the American College of Cardiology 2006;48:446-452. 
Relationship between cardiovascular risk as predicted by established risk scores versus plaque progression as measured by serial intravascular ultrasound von Birgelen C, Hartmann M, Mintz GS, et al.

Circulation 2004;110:1579-1585.

Chapter 6

Spectrum of remodeling behavior observed with serial long-term ( $\geq 12$ months) follow-up intravascular ultrasound studies in left main coronary arteries

von Birgelen C, Hartmann M, Mintz GS, et al.

The American Journal of Cardiology 2004;93:1107-1113.

Chapter 7

Remodeling index compared to actual vascular remodeling in atherosclerotic left main coronary arteries as assessed with long-term ( $\geq 12$ months) serial intravascular ultrasound von Birgelen C, Hartmann M, Mintz GS, et al. Journal of the American College of Cardiology 2006;47:1363-1368.

Chapter 8 129

Relation between baseline plaque burden and subsequent remodeling of atherosclerotic left main coronary arteries: a serial intravascular ultrasound study with long-term ( $\geq 12$ months) follow-up Hartmann M, von Birgelen C, Mintz GS, et al. European Heart Journal 2006;27:1778-1784. 
Dedicated calibration formulas permit correction of differences between measurements by different IVUS devices as demonstrated in atherosclerotic human coronary arteries in vitro

Hartmann M, von Birgelen C, Mintz GS, et al.

The International Journal of Cardiovascular Imaging 2006;22:605-613.

Chapter 10

Reproducibility of volumetric intravascular ultrasound radiofrequency-based analysis of coronary plaque composition in vivo

Hartmann M, Mattern ESK, Huisman J, et al.

The International Journal of Cardiovascular Imaging 2008; in press

Chapter 11 181

Serial intravascular ultrasound of coronary atherosclerosis: an update Hartmann M, Huisman J, Basalus MWZ, et al.

submitted

Summary and conclusions

Samenvatting en conclusies

Acknowledgements

Curriculum vitae

Publications 



\section{Chapter 1}

\section{General Introduction}





\section{Coronary Atherosclerosis}

Atherosclerosis is a major cause of morbidity and mortality in western-lifestyle countries. ${ }^{1-5}$ The term atherosclerosis is of Greek origin and means focal accumulation of lipid [athere] and thickening of the arterial intima [sclerosis]. Coronary artery atherosclerosis refers to the presence of atherosclerotic changes within the walls of the coronary arteries, which may cause impairment or obstruction of coronary blood flow with subsequent myocardial ischemia or infarction. ${ }^{1-7}$ Coronary atherosclerosis is a progressive disease that begins in childhood and leads to clinical manifestation in mid or late adulthood. ${ }^{3}$ Hypercholesterolemia, diabetes mellitus, tabak smoking, genetic disposition, and arterial hypertension are some classical risk factors that promote initiation, development, and progression of coronary atherosclerotic disease. Such risk states can lead to coronary endothelial dysfunction which initiates a complex process that starts with an increased number of low-density-lipoprotein cholesterol particles entering the subendothelial layer. ${ }^{1-5}$ Foam cells form the earliest lesions of atherosclerosis and, over time, the accumulation of cells and matrix results in plaque growth. The distribution of and relation between lipid and connective tissue in the atherosclerotic lesions determines plaques as being stable or at an increased risk of rupture or erosion, which may or may not cause thrombus formation that can lead to clinical sequelae. ${ }^{4,7}$

Therefore, imaging of coronary atherosclerotic plaques may allow the assessment of the mechanisms involved in the course of coronary artery disease (i.e., plaque progression, remodeling, and vulnerability) which could lead to novel surrogate endpoints for the evaluation of anti-atherosclerotic therapies. ${ }^{8-10}$

\section{Coronary Angiography}

Coronary angiography is an x-ray examination of the coronary arteries. A very small tube (catheter) is inserted into a major arterial blood vessel. The tip of the tube is positioned in the origin of the right and left coronary artery and dye is injected. This results in a shadow image (luminogram) of the coronary arteries during the x-ray examination (Figure 1).

Coronary angiography is the standard imaging method for the invasive assessment of coronary artery disease. However, atherosclerosis primarily affects the arterial vessel wall, and atherosclerotic plaque growth (progression) may initially lead to an outward expansion of the vessel wall (positive remodeling). ${ }^{11}$ Therefore, coronary angiography frequently fails to detect the early stages of atherosclerosis, while later stages with significant lumen obstruction can be well 
identified. ${ }^{12-16}$ Importantly, early positively remodeled lesions may not limit coronary blood flow, but may result in acute coronary syndromes as a result of thrombus formation on ruptured or eroded plaques. ${ }^{17-22}$ Coronary angiography provides a two-dimensional view of the arterial luminal silhouette but no visualization of the vessel wall. As a consequence, angiography only provides (indirect) evidence of atherosclerotic disease, if it encroaches the lumen (Figure 1). Vessel foreshortening, irregular plaque distribution and irregular lumen geometry, and overlapping side branches are further factors that impair the accuracy of coronary angiography. ${ }^{13-16}$

\section{Intravascular Ultrasound - Technique}

Intravascular ultrasound (IVUS) is a catheter-based diagnostic method that provides real-time, high-resolution, tomographic images of both, coronary lumen and vessel wall. ${ }^{23,24}$ The coronary artery is selectively cannulated by a catheter that incorporates a miniature transducer (diameter approximately $1 \mathrm{~mm}$ ) which emits and receives high-frequency ultrasound (usually in the range of 20 to $45 \mathrm{MHz}$ ). As the transducer is moved through the artery, ultrasonic reflections are electronically converted to obtain cross-sectional images (Figure 1).
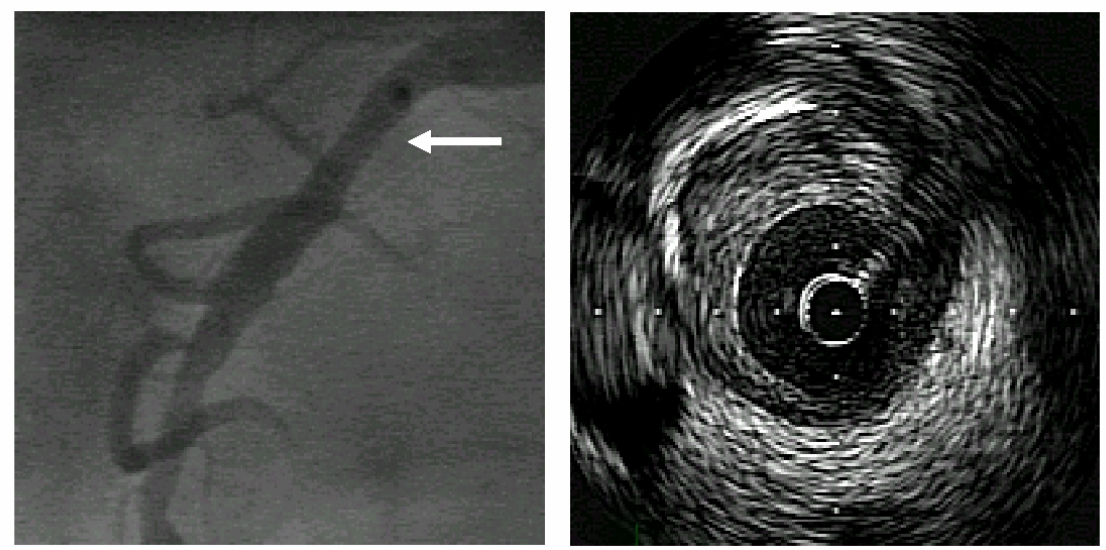

Figure 1. Coronary angiography provides a luminogram of the coronary artery and allows an indirect assessment of at least mild-to-moderate coronary atherosclerosis (left). Intravascular ultrasound (IVUS) provides detailed information about the coronary artery lumen and vessel wall. An eccentric atherosclerotic plaque in the proximal part of the right coronary artery (arrowhead in angiogram) is visualized with IVUS (right). 
Morphology, severity, and dimensions of coronary atherosclerotic plaques can be assessed with IVUS. ${ }^{23-25}$ There are basically two types of commercially available IVUS imaging catheters: (1) a mechanical system that contains a flexible imaging cable that rotates a single transducer at its distal tip inside a echolucent distal sheath, and (2) an electronic (solid-state) catheter system with multiple imaging elements at its distal tip, providing cross-sectional images by sequentially activating the imaging elements in a circular way. ${ }^{23,24}$ IVUS is widely applied for the assessment of coronary atherosclerosis and for the guiding of percutaneous interventions with a good short and long-term saftey. ${ }^{28,29}$

\section{Intravascular Ultrasound - Qualitative and Quantitative Measurements}

Qualitative and quantitative IVUS analyses should be performed according to the American College of Cardiology Clinical Expert Consensus Document on Standards for Acquisition, Measurement and Reporting of Intravascular Ultrasound Studies. ${ }^{24}$ Contour detection at the leading edge of the lumen and the mediaadventitia interface allows the assessment of two direct measurements: the lumen and the total vessel cross-sectional area. The difference between total vessel and lumen area is the plaque plus media cross-sectional area, which is a measure of the atherosclerotic plaque. ${ }^{23,24}$ The quantitative IVUS measurements are shown in Figure 2.
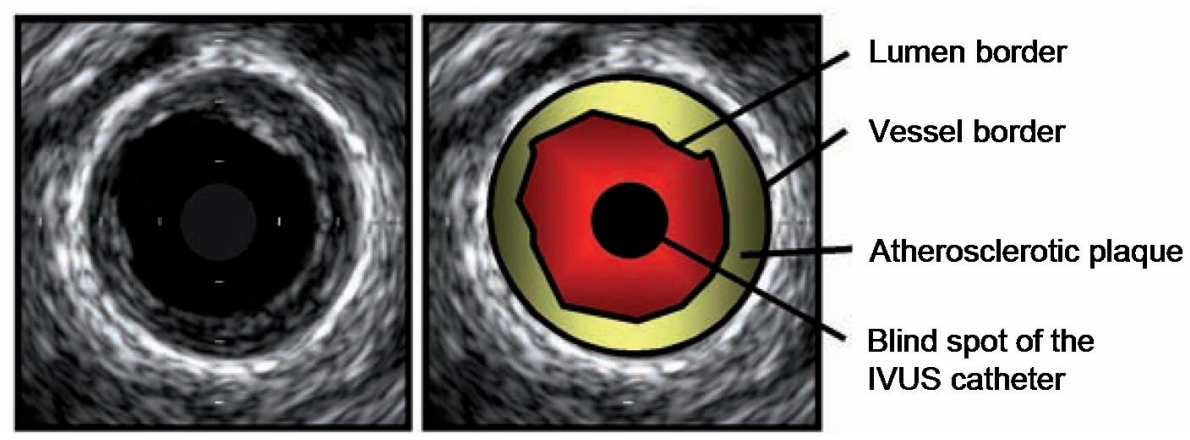

Figure 2. Quantitative intravascular ultrasound (IVUS) measurements.

Visual assessment of the predominant plaque composition characterizes atherosclerotic plaques as soft (low echogenicity), fibrous (high echogenicity), calcified (high echogenicity with acoustic shadowing and/or reverberations), or mixed. ${ }^{23,24}$ Quantitative IVUS measurements are highly reproducible and therefore 
suitable for a serial study design as demonstrated in previous validation studies. $^{26,27,30-33}$

\section{Intravascular Ultrasound - Coronary Atherosclerosis Progression-Regression}

Cardiovascular events occur as a result of coronary atherosclerotic plaque formation and progression. ${ }^{5-8}$ Modification of cardiovascular risk factors (e.g., cholesterol-lowering) significantly improves clinical outcome as demonstrated in large-scale, multicenter-trials with long-term follow-up. ${ }^{34,35}$ IVUS allows direct quantification of coronary vessel dimensions and may help to understand the relation between cardiovascular risk factors and atherosclerotic plaque phenotypes. ${ }^{9,32}$ IVUS derived plaque measurements could serve as a surrogate endpoint and may therefore offer the possibility to test novel anti-atherosclerotic therapies in smaller groups of patients with shorter follow-up. ${ }^{9,10}$ Nevertheless, the value of IVUS as a surrogate endpoint has still to be determined in ongoing large trials.

IVUS assessment of an atherosclerotic plaque at a single point in time may not reflect the rate of plaque progression during the following period of time as coronary atherosclerosis is a dynamic process. Single point observations are not able to characterize the "dynamic status" of coronary atherosclerosis. ${ }^{24}$ Serial assessment of coronary plaques should be the gold standard when analyzing the relation between cardiovascular risk factors and coronary atherosclerosis. ${ }^{24}$ Moreover, serial IVUS has the advantage of permitting the assessment of potential interaction between changes in athersclerotic plaque, luminal, and vessel dimension. ${ }^{23,24}$ Several interesting questions could be answered:

- Is there a relation between cardiovascular risk factors and IVUS assessed changes of plaque dimensions ?

- Which risk factors are predictors of IVUS plaque progression ?

- Are there thresholds (e.g., serum cholesterol levels) which are associated with a stop of atherosclerosis progression or even regression by IVUS ?

- Does the IVUS derived progression-rate reflect estimated cardiovascular risk or actual coronary events?

\section{Intravascular Ultrasound - Coronary Arterial Remodeling}

In 1978 Seymour Glagov described the phenomenon of vascular remodeling by examining sectioned left main coronary arteries from necropsy specimens. ${ }^{11} \mathrm{He}$ observed local increase in total vessel size that was proportional to the amount of 
atherosclerotic plaque burden. Glagov's observations suggest that coronary arteries may change size to adapt to plaque accumulation. The relationship between vessel size and plaque burden was given up to a plaque burden of $40 \%$; thereafter the lumen became compromised due to the inability of the artery to further expand. This process of arterial enlargement to accommodate the plaque and maintain the luminal dimension is called Glagov phenomenon, compensatory enlargement, or (more commonly) positive vascular remodeling. ${ }^{11}$

IVUS allows the real-time assessment of the lumen, plaque, and total vessel dimensions and the examination of focal areas of arterial expansion associated with focal accumulation of plaque. Early non-serial IVUS studies replicated Glagov's observations and the concept of positive remodeling. ${ }^{36-38}$ A remodeling index was developed, which compares the vessel area at reference sites to the vessel area at lesion site. ${ }^{24}$ Importantly, IVUS extended the concept of vascular remodeling beyond the Glagovian compensatory enlargement by showing focal luminal narrowing (negative remodeling) at sides of reduced total vessel dimensions. ${ }^{39-42}$ This demonstrated that luminal stenosis can result from arterial shrinkage in addition to atherosclerotic plaque accumulation. A remodeling index (lesion vessel divided by reference vessel area) greater than 1 indicates positive remodeling and a remodeling index smaller than 1 indicates negative remodeling. ${ }^{24}$ Different types of remodeling pattern are shown in Figure 3.
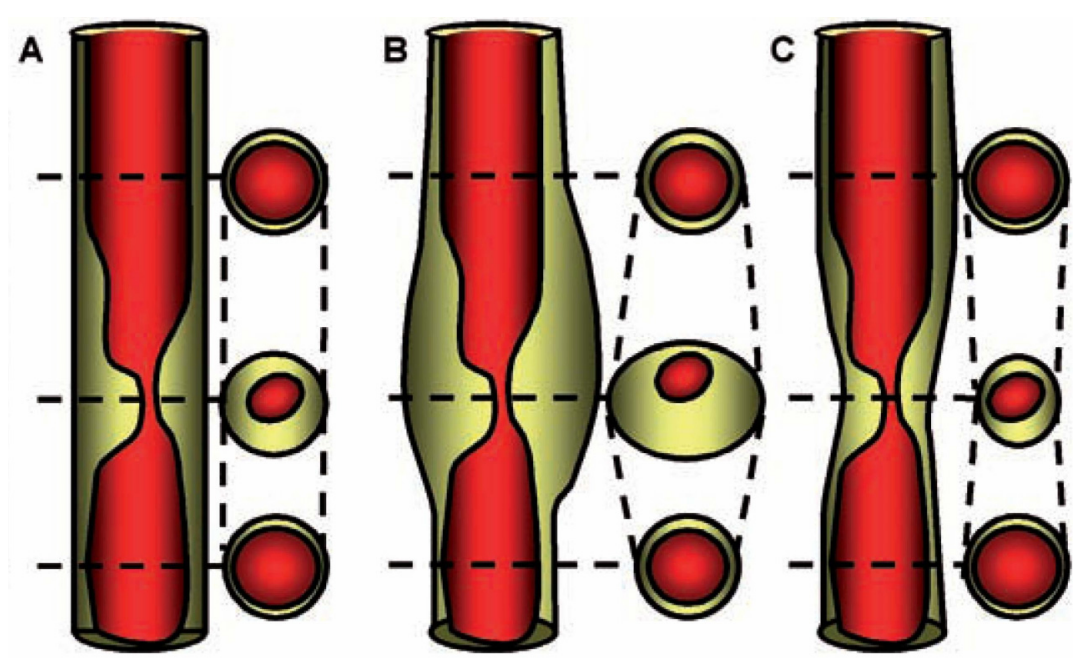

Figure 3. Differences in remodeling behaviour. An atherosclerotic coronary lesion without vascular remodeling $(A)$; with positive vascular remodeling $(B)$, and with negative vascular remodeling $(C)$. 
The IVUS assessment of the remodeling state of coronary lesions has important clinical implications, as the remodeling state is related to plaque vulnerability and acute coronary syndromes; in addition, coronary remodeling is a predictor of complications related to percutaneous coronary interventions. ${ }^{43-50}$

Nevertheless, Glagov's observation and the IVUS concept of positive and negative remodeling are only indirect evidence of a dynamic process. Direct evidence of arterial remodeling can only be obtained if coronary lesions are followed with IVUS over time (serial IVUS examinations). ${ }^{24,51}$ Serial changes in vessel area that go along with changes in plaque area would definitively prove the presence of remodeling. ${ }^{51}$ Such serial IVUS assessment could answer the following questions:

- Do early lesions more often show positive or negative remodeling ?

- Is positive versus negative remodeling related to patient-specific or lesionspecific characteristics?

- Do lesions change from one type of remodeling to another ?

- Does the remodeling-index display the true serial remodeling behaviour?

\section{Intravascular Ultrasound - Atherosclerotic Plaque Vulnerability}

Rupture of a vulnerable atherosclerotic plaque is the cause of most acute coronary syndromes. Atherosclerotic plaque vulnerability is related to the histological plaque composition. 2,4,5,52 Conventional grey-scale IVUS is a useful method for characterizing extent and distribution of atherosclerotic plaques. ${ }^{23-27}$ However, the region of low echogenicity in grey-scale IVUS images, which is thought to represent the composition of lipid-containing and mixed plaque (a potential marker of plaque vulnerability), remains relatively uncharacterized by grey-scale IVUS. ${ }^{53}$

Spectral analysis of the radiofrequency ultrasound backscatter signals offers in vivo the opportunity to better assess plaque morphology. ${ }^{54,55}$ The radiofrequencybased IVUS technology has been shown to have an $80 \%$ to $92 \%$ accuracy when used to identify the four different types of atherosclerotic plaque components (i.e., fibrous, fibro-lipidic, and necrotic core tissue, and calcium) ${ }^{54,55}$ In addition, the detection of vulnerable plaques can be achieved in vivo. ${ }^{56}$ Radiofrequency-based IVUS may offer the potential to serially assess changes in plaque composition beside changes in plaque geometry. However, an important prerequisite of the use of changes in radiofrequency-based IVUS data as a surrogate endpoint of serial studies is a sufficient measurement reproducibility. This may be particularly important as the effect of pharmacological anti-atherosclerotic therapies on plaque dimensions and composition may be relatively small. ${ }^{53,57}$ 


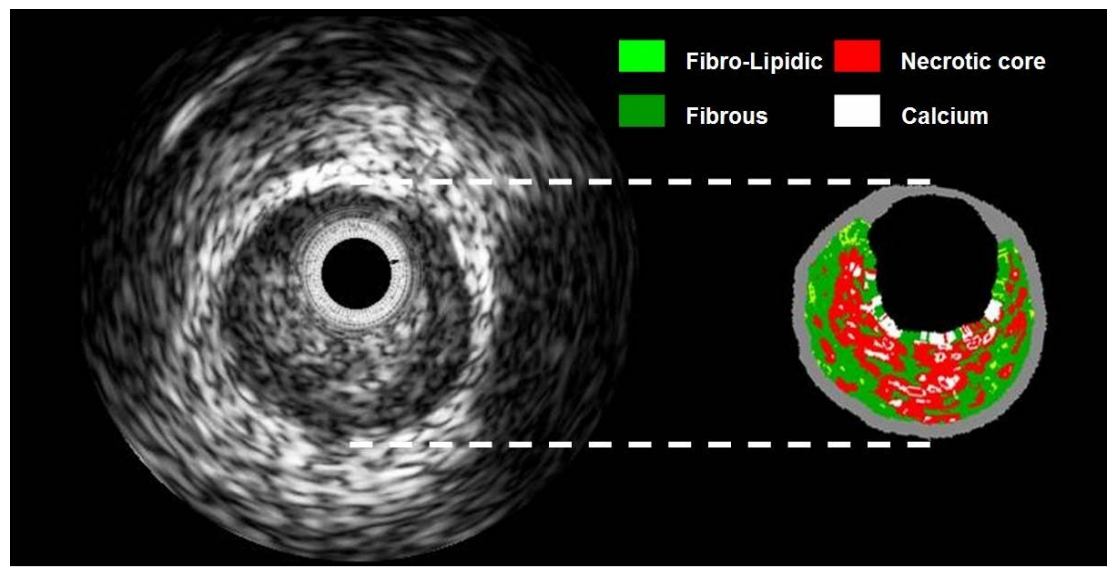

Figure 4. Radiofrequency-based intravascular ultrasound (IVUS) analysis of atherosclerotic plaque tissue components. On the left, a conventional gray-scale IVUS image is shown while the right image displays the corresponding radiofrequency-based IVUS analysis of plaque composition.

\section{Aim of this Thesis}

With non-serial IVUS studies much knowledge has been gained about coronary atherosclerosis. The aim of this thesis was to further investigate the role of serial IVUS in the assessment of atherosclerotic plaque progression and remodeling, and finally to test the potential of novel IVUS technologies for serial plaque assessment.

- In Chapter 2 we assessed the relation between serum cholesterol levels and progression-regression of left main coronary atherosclerosis.

- In Chapter 3 we further investigated the relation between serum cholesterol levels and plaque progression-regression at different stages of age.

- In Chapter 4 we investigated the relation between classic and novel cardiovascular risk factors (e.g., lipoprotein(a)) and plaque progression.

- In Chapter 5 we explored the relation between IVUS assessed plaque progression and the estimated risk of cardiovascular events as well as actual coronary events.

- In Chapter 6 we analyzed the serial remodeling behaviour of left main atherosclerotic plaques and interrelations between changes in lumen, plaque, and vessel dimensions.

- In Chapter 7 we validated the non-serial measure of coronary remodeling (remodeling index) with the actual (serial) remodeling behaviour. 
- In Chapter 8 we compared Glagov's remodeling concept with the serial remodeling behaviour of atherosclerotic plaques with different amounts of plaque burden.

- In Chapter 9 we compared the measurement differences of mechanical and electronical IVUS systems in vitro and validated the application of dedicated correction formulas.

- In Chapter 10 we tested the reproducibility of radiofrequency-based volumetric IVUS measurements in atherosclerotic coronary segments.

- In Chapter 11 we give an up-date on the current knowledge that has been gained from various serial IVUS studies of coronary atherosclerosis.

\section{References}

1. Virchow R. Cellular pathology as based upon physiological and pathological histology. Lecture XVI - atheromatous affection of arteries. Published by Hirschwald, Berlin 1858.

2. Fuster V, Badimon L, Badimon JJ, et al. The pathogenesis of coronary artery disease and the acute coronary syndromes. N Engl J Med 1992;326:242-250.

3. Stary HC. Changes in components and structure of atherosclerotic lesions developing from childhood to middle age in coronary arteries. Basic Res Cardiol 1993;89:17-32.

4. Libby P. Current concepts of the pathogenesis of the acute coronary syndromes. Circulation 2001;104:365-372.

5. Shah PK. Mechanisms of plaque vulnerability and rupture. J Am Coll Cardiol 2003;41:15-22.

6. Waters D, Craven TE, Lesperance J. Prognostic significance of progression of coronary atherosclerosis. Circulation 1993;87:1399-1401.

7. Burke AP, Farb A, Malcom GT, et al. Coronary risk factors and plaque morphology in men with coronary disease who died suddenly. N Engl J Med 1997:336:1276-1282.

8. Ambrose JA, Tannenbaum MA, Alexopoulos D, et al. Angiographic progression of coronary artery disease and the development of myocardial infarction. J Am Coll Cardiol 1988;12:56-62.

9. Böse D, von Birgelen C, Erbel R. Intravascular ultrasound for the evaluation of therapies targeting coronary atherosclerosis. J Am Coll Cardiol 2007;49:925-932.

10. Kastelein $\mathrm{JJ}$, de Groot E. Ultrasound imaging techniques for the evaluation of cardiovascular therapies. Eur Heart J 2008;29:849-858.

11. Glagov S, Weisenberg E, Zarins CK, et al. Compensatory enlargement of human atherosclerotic coronary arteries. N Engl J Med 1987;316:1371-1375.

12. Grondin CM, Dyrda I, Pasternac A, et al. Discrepancies between cineangiographic and postmortem findings in patients with coronary artery disease and recent myocardial revascularization. Circulation 1974;49:703-708.

13. de Feyter PJ, Serruys PW, Davies MJ, et al. Quantitative coronary angiography to measure progression and regression of coronary atherosclerosis. Value, limitations, and implications for clinical trials. Circulation 1991;84:412-423.

14. Alfonso F, Macaya C, Goicolea J, et al. Intravascular ultrasound imaging of angiographically normal coronary segments in patients with coronary artery disease. Am Heart J 1994;127:536-44.

15. Ge J, Liu F, Görge G, et al. Angiographically 'silent' plaque in the left main coronary artery detected by intravascular ultrasound. Coron Artery Dis 1995;6:805-810.

16. Ricciardi MJ, Meyers S, Choi K, et al. Angiographically silent left main disease detected by 
intravascular ultrasound: a marker for future adverse cardiac events. Am Heart J 2003;146:507512.

17. Pasterkamp G, Schoneveld $\mathrm{AH}$, van der Wal AC, et al. Relation of arterial geometry to luminal narrowing and histologic markers for plaque vulnerability: the remodeling paradox. J Am Coll Cardiol 1998;32:655-662.

18. Schoenhagen $\mathrm{P}$, Ziada KM, Kapadia SR, et al. Extent and direction of arterial remodeling in stable versus unstable coronary syndromes: an intravascular ultrasound study. Circulation 2000;101:598603.

19. von Birgelen C, Klinkhart W, Mintz GS, et al. Plaque distribution and vascular remodeling of ruptured and nonruptured coronary plaques in the same vessel: an intravascular ultrasound study in vivo. J Am Coll Cardiol 2001;37:1864-1870.

20. Nakamura M, Nishikawa H, Mukai S, et al. Impact of coronary artery remodeling on clinical presentation of coronary artery disease: an intravascular ultrasound study. J Am Coll Cardiol 2001;37:63-69.

21. Schoenhagen $P$, Ziada KM, Vince DG, et al. Arterial remodeling and coronary artery disease: the concept of "dilated" versus "obstructive" coronary atherosclerosis. J Am Coll Cardiol 2001;38:297306.

22. Varnava AM, Mills PG, Davies MJ. Relationship between coronary artery remodeling and plaque vulnerability. Circulation 2002;105:939-943.

23. Di Mario C, Görge G, Peters R, et al. Clinical application and image interpretation in intracoronary ultrasound. Study group on intracoronary imaging of the working group of coronary circulation and of the subgroup on intravascular ultrasound of the working group of echocardiography of the European Society of Cardiology. Eur Heart J 1998;19:207-229.

24. Mintz GS, Nissen SE, Anderson WD, et al. American College of Cardiology clinical expert consensus document on standards for acquisition, measurement and reporting of intravascular ultrasound studies (IVUS). J Am Coll Cardiol 2001;37:1478-1492.

25. Potkin BN, Bartorelli AL, Gessert JM, et al. Coronary artery imaging with intravascular highfrequency ultrasound. Circulation 1992;68:276-281.

26. Nishimura RA, Edwards WD, Warnes CA, et al. Intravascular ultrasound imaging: in vitro validation and pathologic correlation. J Am Coll Cardiol 1990;16:145-154.

27. Bartorelli AL, Neville RF, Keren G, et al. In vitro and in vivo intravascular ultrasound. Eur Heart $J$ 1992;13:102-108.

28. Gaster AL, Slothuus Skjoldborg U, Larsen J, et al. Continued improvement of clinical outcome and cost effectiveness following intravascular ultrasound guided $\mathrm{PCl}$ : insights from a prospective, randomised study. Heart. 2003;89:1043-1049

29. Guédès $A$, Keller PF, L'Allier PL, et al. Long-term safety of intravascular ultrasound in nontransplant, nonintervened, atherosclerotic coronary arteries. J Am Coll Cardiol 2005 15;45:559564.

30. von Birgelen C, van der Lugt A, Nicosia A, et al. Computerized assessment of coronary lumen and atherosclerotic plaque dimensions in three-dimensional intravascular ultrasound correlated with histomorphometry. Am J Cardiol 1996;78:1202-1209.

31. von Birgelen C, de Vrey EA, Mintz GS, et al. ECG-gated three-dimensional intravascular ultrasound: feasibility and reproducibility of the automated analysis of coronary lumen and atherosclerotic plaque dimensions in humans. Circulation 1997;96:2944-2952.

32. von Birgelen C, Slager CJ, Di Mario C, et al. Volumetric intracoronary ultrasound: a new maximum confidence approach for the quantitative assessment of progression-regression of atherosclerosis? Atherosclerosis 1995;118:103-113.

33. Foster GP, Mittleman MA, Koch M, et al. Variability in the measurement of intracoronary ultrasound images: implications for the identification of atherosclerotic plaque regression. Clin Cardiol 1997;20:11-15. 
34. Larosa JC, Grundy SM, Waters DD, et al. Intensive lipid lowering with atorvastatin in patients with stable coronary disease. N Engl J Med 2005;352:1425-1435.

35. Cannon $\mathrm{CP}$, Braunwald $\mathrm{E}, \mathrm{McC}$ abe $\mathrm{CH}$, et al. Intensive versus moderate lipid lowering with statins after acute coronary syndromes. N Engl J Med 2004;350:1495-1504.

36. Hermiller JB, Tenaglia AN, Kisslo KB, et al. In vivo validation of compensatory enlargement of atherosclerotic coronary arteries. Am J Cardiol 1993;71:665-668.

37. Losordo DW, Rosenfield K, Kaufman J, et al. Focal compensatory enlargement of human arteries in response to progressive atherosclerosis. In vivo documentation using intravascular ultrasound. Circulation 1994;89:2570-2577.

38. Ge J, Erbel R, Zamorano J, Koch L, et al. Coronary artery remodeling in atherosclerotic disease: an intravascular ultrasonic study in vivo. Coron Artery Dis 1993;4:981-986.

39. Nishioka T, Luo H, Eigler NL, et al. Contribution of inadequate compensatory enlargement to development of human coronary artery stenosis: an in vivo intravascular ultrasound study. J Am Coll Cardiol 1996;27:1571-1576.

40. von Birgelen C, Airiian SG, Mintz GS, et al. Variations of remodeling in response to left main atherosclerosis assessed with intravascular ultrasound in vivo. Am J Cardiol 1997;80:1408-1413.

41. Pasterkamp G, Wensing PJ, Post MJ, et al. Paradoxical arterial wall shrinkage may contribute to luminal narrowing of human atherosclerotic femoral arteries. Circulation 1995;91:1444-1449.

42. Mintz GS, Kent KM, Pichard AD, et al. Contribution of inadequate arterial remodeling to the development of focal coronary artery stenoses: an intravascular ultrasound study. Circulation 1997;95:1791-1798.

43. Fujii K, Kobayashi Y, Mintz GS, et al. Intravascular ultrasound assessment of ulcerated ruptured plaques: a comparison of culprit and nonculprit lesions of patients with acute coronary syndromes and lesions in patients without acute coronary syndromes. Circulation 2003;108:2473-2478.

44. Kotani J, Mintz GS, Castagna MT, et al. Intravascular ultrasound analysis of infarct-related and non-infarct-related arteries in patients who presented with an acute myocardial infarction. Circulation 2003;107:2889-2893.

45. Gyöngyösi $M$, Wexberg $P$, Kiss $K$, et al. Adaptive remodeling of the infarct-related artery is associated with recurrent ischemic events after thrombolysis in acute myocardial infarction. Coron Artery Dis 2001;12:167-172.

46. Dangas G, Mintz GS, Mehran R, et al. Preintervention arterial remodeling as an independent predictor of target-lesion revascularization after nonstent coronary intervention: an analysis of 777 lesions with intravascular ultrasound imaging. Circulation 1999;99:3149-3154.

47. Okura $\mathrm{H}$, Morino $\mathrm{Y}$, Oshima $\mathrm{A}$, et al. Preintervention arterial remodeling affects clinical outcome following stenting: an intravascular ultrasound study. J Am Coll Cardiol 2001;37:1031-1035.

48. Sahara $M$, Kirigaya $H$, Oikawa $Y$, et al. Arterial remodeling patterns before intervention predict diffuse in-stent restenosis: an intravascular ultrasound study. J Am Coll Cardiol 2003;42:1731-1738.

49. Mintz GS, Tinana A, Hong MK, et al. Impact of preinterventional arterial remodeling on neointimal hyperplasia after implantation of (non-polymer-encapsulated) paclitaxel-coated stents: a serial volumetric intravascular ultrasound analysis from the ASian Paclitaxel-Eluting Stent Clinical Trial (ASPECT). Circulation 2003;108:1295-1298.

50. Wexberg P, Gyöngyösi M, Sperker W, et al. Pre-existing arterial remodeling is associated with inhospital and late adverse cardiac events after coronary interventions in patients with stable angina pectoris. J Am Coll Cardiol 2000;36:1860-1869.

51. Shiran A, Mintz GS, Leiboff B, et al. Serial volumetric intravascular ultrasound assessment of arterial remodeling in left main coronary artery disease. Am J Cardiol 1999;83:1427-1432.

52. Virmani R, Burke AP, Farb A, et al. Pathology of the vulnerable plaque. J Am Coll Cardiol 2006;47:C13-C18.

53. Mehta SK, McCrary JR, Frutkin AD, et al. Intravascular ultrasound radiofrequency analysis of coronary atherosclerosis: an emerging technology for assessment of vulnerable plaque. Eur Heart 
J 2007;28:1283-1288.

54. Nair A, Kuban BD, Tuzcu EM, et al. Coronary plaque classification with intravascular ultrasound radiofrequency data analysis. Circulation 2002;106:2200-2206.

55. Nasu K, Tsuchikane E, Katoh $\mathrm{O}$, et al. Accuracy of in vivo coronary plaque morphology assessment: a validation study of in vivo virtual histology compared with in vitro histopathology. $\mathrm{J}$ Am Coll Cardiol 2006;47:2405-2412.

56. Rodriguez-Granillo GA, García-García HM, McFadden EP, et al. In vivo intravascular ultrasoundderived thin-cap fibroatheroma detection using ultrasound radiofrequency data analysis. J Am Coll Cardiol 2005;46:2038-2042.

57. Ambrose JA, D'Agate DJ. Classification of systemic therapies for potential stabilization of the vulnerable plaque to prevent acute myocardial infarction. Am J Cardiol 2005;95:379-382. 



\section{Chapter 2}

Relation Between Progression and Regression of Atherosclerotic Left Main Coronary Artery Disease and Serum Cholesterol Levels as Assessed With

Serial Long-Term ( $\geq 12$ Months) Follow-Up Intravascular Ultrasound

Clemens von Birgelen, Marc Hartmann, Gary S. Mintz, Dietrich Baumgart, Axel Schmermund, Raimund Erbel

Reprinted with permission from:

Circulation 2003;108(22):2757-2762.

๑) 2003 American Heart Association / Lippincott Williams \& Wilkins Inc. 



\section{Abstract}

Background: The relation between serum lipids and risk of coronary events has been established, but there are no data demonstrating directly the relation between serum low-density lipoprotein (LDL) cholesterol and high-density lipoprotein (HDL) cholesterol versus serial changes in coronary plaque dimensions.

Methods and Results: We performed standard analyses of serial intravascular ultrasound (IVUS) studies of 60 left main coronary arteries obtained 18.3 \pm 9.4 months apart to evaluate progression and regression of mild atherosclerotic plaques in relation to serum cholesterol levels. Overall, there was (1) a positive linear relation between LDL cholesterol and the annual changes in plaque plus media (P\&M) cross-sectional area (CSA) $(r=0.41, P<0.0001)$ with $(2)$ an LDL value of 75 $\mathrm{mg} / \mathrm{dL}$ as the cutoff when regression analysis predicted on average no annual P\&M CSA increase; (3) an inverse relation between HDL cholesterol and annual changes in P\&M CSA ( $r=-$ $0.30, P<0.02)$; (4) an inverse relation between LDL cholesterol and annual changes in lumen CSA $(r=-0.32, P<0.01)$; and (5) no relation between LDL and HDL cholesterol and the annual changes in total arterial CSA (remodeling). Despite similar baseline IVUS characteristics, patients with an LDL cholesterol level $\geq 120 \mathrm{mg} / \mathrm{dL}$ showed more annual P\&M CSA progression and lumen reduction than patients with lower LDL cholesterol.

Conclusions: There is a positive linear relation between LDL cholesterol and annual changes in plaque size, with an LDL value of $75 \mathrm{mg} / \mathrm{dL}$ predicting, on average, no plaque progression. HDL cholesterol shows an inverse relation with annual changes in plaque size.

\section{Introduction}

The relation between serum lipids and coronary events has been established in patients with ${ }^{1,2}$ and without ${ }^{3,4}$ overt coronary artery disease. Clinical events occur as a result of atherosclerotic plaque formation and progression. ${ }^{5,6}$ Serial examinations of plaques are particularly important, because they may allow insights into the mechanisms involved. Thus far, in coronary arteries, the relationship of risk factors, in particular serum lipids, to actual plaque progression (increase) or regression (decrease) has been inferred from indirect assessment of the coronary calcium score by cardiac computer tomography or angiography. 7,8 However, angiographic studies suggested only minimal lumen changes, often too small and occurring too slowly to account for the observed early clinical benefit of lipid-lowering strategies. ${ }^{8}$

Intravascular ultrasound (IVUS) allows transmural visualization of coronary arteries and direct measurements of lumen, plaque, and vessel dimensions. ${ }^{9-12}$ As a consequence, IVUS is an ideal tool for the assessment of the mechanisms that may be involved in the progression or regression of coronary artery disease. Nevertheless, the literature contains few serial IVUS studies of native coronary artery disease (mostly with 6 to 12 months of follow-up). ${ }^{13-15}$ Only 1 serial IVUS pharmacological intervention study in 25 patients investigated the evolution of coronary artery disease in native (non-transplanted) coronary arteries with $>1$-year 
follow-up. ${ }^{13}$ The left main (LM) coronary artery may be the most important target of atherosclerotic plaque accumulation, ${ }^{16}$ and IVUS often reveals occult plaque. ${ }^{11,12}$

In the present study, we analyzed serial IVUS data of nonstenotic LM coronary arteries in patients with IVUS follow-up of $\geq 12$ months. We compared changes in lumen, plaque, and arterial dimensions in relation to serum lipids, in particular, versus both LDL cholesterol and HDL cholesterol levels.

\section{Methods}

\section{Study Population}

We analyzed serial IVUS studies of 60 LM coronary artery atherosclerotic plaques during $\geq 12$ months of follow-up (18.3 \pm 9.4 months). All patients were examined in the Essen University Cardiac Catheterization Laboratory. All plaques were de novo, were hemodynamically nonsignificant, and met the following criteria: (1) serial highquality IVUS of the entire LM $\geq 12$ months apart, (2) calcifications that did not limit quantitative assessment of vessel cross-sectional area (CSA), (3) nonostial plaque location, (4) angiographic lumen diameter stenosis <30\% ("worst view" visual assessment), and (5) no intervention in the very proximal left anterior descending or circumflex coronary arteries, because these interventions could have affected the LM artery. This IVUS study was approved by the Local Council on Human Research. All patients signed a written informed consent form as approved by the Local Medical Ethics Committee.

\section{Cardiovascular Risk Factors, Clinical Data, and Medication}

In our laboratory, we prospectively record demographics, cardiovascular risk factors, medications, and results of key laboratory tests of patients examined with IVUS. All laboratory tests were performed at baseline and follow-up as part of the clinical routine and were analyzed in the central laboratory of Essen University according to international standards. Cardiovascular risk factors that were recorded included diabetes mellitus and hypertension (both medication-dependent only), hypercholesterolemia (medication-dependent, total serum cholesterol $>200 \mathrm{mg} / \mathrm{dL}$, or LDL cholesterol $>160 \mathrm{mg} / \mathrm{dL}$ ), history of smoking, and family history of coronary artery disease. Data of laboratory tests were means of the baseline and follow-up values. Medications were recorded only if drugs were taken for $>50 \%$ of the followup interval (eg, clopidogrel for 4 weeks was not tabulated). Plasma concentrations of total cholesterol, LDL cholesterol, HDL cholesterol, and triglycerides were measured by standard enzymatic methods, and the LDL/HDL ratio was calculated. 


\section{IVUS Imaging Protocol}

IVUS imaging was initially performed during percutaneous coronary interventions of mid or distal left anterior or left circumflex arteries. IVUS studies were performed after intracoronary injections of $200 \mu \mathrm{g}$ nitroglycerin with commercially available systems; a mechanical sector scanner (Boston Scientific Corp) incorporating a 30$\mathrm{MHz}$ single-element beveled transducer or a solid-state device (Endosonics). Importantly, at Essen University, if a patient undergoes imaging with one IVUS system during an index procedure, the same IVUS system is used at follow-up. Slow, continuous pullbacks of the IVUS transducer were started as distal as possible in one of the left coronary arteries and were generally performed using a motorized pullback device (at $0.5 \mathrm{~mm} / \mathrm{s}$ ). IVUS images of the entire pullback were recorded on 0.5 -in high-resolution s-VHS tape. In addition, a dedicated image-inimage system (Echo-Map, Siemens) ${ }^{17}$ was used to record the "angiographic" position of the IVUS probe together with the corresponding IVUS image, especially at sites of characteristic landmarks (ie, calcifications or unusual plaque shapes) and/or the target site.

Follow-up IVUS studies were performed (using the same IVUS system as initially) during repeat coronary interventions $(n=34,57 \%)$ and during IVUS examinations of ambiguous coronary lesions or (clinically driven) follow-up catheterizations $(n=26,43 \%)$. IVUS was not performed as part of another study with long-term IVUS follow-up in any of these patients.

\section{Quantitative IVUS Analysis}

The LM target site image slice was determined from the initial IVUS study; this was the site with the smallest lumen CSA within the LM plaque. If there were several slices with equal lumen size, the one with the largest external elastic membrane (EEM) and plaque-plus-media (P\&M=EEM minus lumen) CSA was analyzed..$^{9,12}$ Exact matching of the target site on initial and follow-up IVUS studies was ensured by use of side-by-side comparison of the serial IVUS video sequences along with information of the pullback speed; the operators' recorded comments (on videotape); and characteristic calcifications, vascular and perivascular landmarks, and plaque shapes. If required, $x$-ray sequences of the dedicated image-in-image system (Echo-Map) were revisited to optimize matching. ${ }^{17}$

The lumen CSA was measured by tracing the leading edge of the intima. The EEM CSA was measured by tracing the leading edge of the adventitia. In our laboratory, the intraclass correlation coefficient is 0.99 for repeated measurements of EEM, 0.96 for lumen, and 0.99 for P\&M CSA. Plaque burden (\%) was calculated as (P\&M divided by EEM) $\times 100 \%$. To compensate for variations in follow-up 
intervals and to obtain comparable data, we calculated absolute and relative changes $(\Delta)$ between initial and follow-up IVUS data; measurements were normalized for the length of the follow-up period (changes per year) and for baseline measurements. In analogy with 1 previous coronary progressionregression study, ${ }^{18}$ we used an $\mathrm{LDL}$ cholesterol threshold of $120 \mathrm{mg} / \mathrm{dL}$ to compare patients with $\mathrm{LDL}$ cholesterol $\geq 120 \mathrm{mg} / \mathrm{dL}$ (group A) versus those with $\mathrm{LDL}$ cholesterol $<120 \mathrm{mg} / \mathrm{dL}$ (group B).

\section{IVUS Assessment of Plaque Composition}

IVUS images were read offline by 3 experienced IVUS analysts. Plaque composition was assessed visually as previously described. ${ }^{9}$ The arc of targetlesion calcium $\left({ }^{\circ}\right)$ was measured with a protractor centered on the lumen; if necessary, the total arc of calcium was obtained by adding arcs of individual deposits. Plaques were classified as calcified if the total arc of lesion calcium was $>180^{\circ}$. Extrapolation of the EEM boundary behind calcium was possible if each individual calcific deposit did not shadow $>75^{\circ}$ of the adventitial circumference.

\section{Statistical Analysis}

Analyses were performed with SPSS 10.0.7 (Microsoft) for Windows. Dichotomous data are presented as frequencies and compared by use of Chi-square statistics or Fisher's exact test. Quantitative data are presented as mean $\pm S D$ and compared by Student's $\mathrm{t}$ test and regression analysis. A probability value of $P<0.05$ was considered significant.

\section{Results}

Demographics, Medication, and Laboratory Testing of Patients

Twenty-six patients $(43 \%)$ had a serum LDL cholesterol level $\geq 120 \mathrm{mg} / \mathrm{dL}$ (group A); 34 patients $(57 \%$ ) had LDL cholesterol values $<120 \mathrm{mg} / \mathrm{dL}$ (group B). Demographics of both groups (all white) are presented in Table 1. Group A patients tended to have more systemic arterial hypertension. The medications of groups $A$ and $B$ were not different except for a higher incidence of statin use in group $B$ (Table 2; $P<0.0005$ ).

In keeping with the definitions, group $A$ patients had higher serum LDL cholesterol values (Table $1 ; P<0.0001$ ). In addition, group $A$ patients had greater total cholesterol $(P<0.0001)$, lipoprotein $(a)(p<0.05)$, apolipoprotein $B(P<0.0005)$, 
and fibrinogen values $(P<0.05)$. Group $B$ patients showed a higher HDL cholesterol $(P<0.01)$. Triglycerides values were similar in groups $\mathrm{A}$ and $\mathrm{B}$.

Table 1 (part 1). Patient Demographics, Medications, and Laboratory Tests.

\begin{tabular}{|c|c|c|c|}
\hline & $\begin{array}{c}\text { Group A } \\
(n=26)\end{array}$ & $\begin{array}{c}\text { Group B } \\
(n=34)\end{array}$ & $\boldsymbol{P}$ \\
\hline Time of follow-up, mo & $16.4 \pm 6.2$ & $19.6 \pm 11.1$ & 0.2 \\
\hline Age, y & $59 \pm 10$ & $58 \pm 9$ & 0.9 \\
\hline Men & $21(81)$ & $29(85)$ & 0.7 \\
\hline Body mass index, $\mathrm{kg} / \mathrm{m}^{2}$ & $27.4 \pm 3.5$ & $26.2 \pm 3.0$ & 0.2 \\
\hline Previous myocardial infarction & $8(31)$ & $13(38)$ & 0.7 \\
\hline Hypercholesterolemia & $20(77)$ & $8(24)$ & $<0.0001$ \\
\hline Systemic arterial hypertension & $23(86)$ & $20(59)$ & 0.05 \\
\hline Diabetes & $4(15)$ & $4(12)$ & 0.7 \\
\hline Smoker & $7(27)$ & $9(27)$ & 0.8 \\
\hline Family history of coronary artery disease & $6(23)$ & $8(24)$ & 0.8 \\
\hline No. of vessels diseased & & & 0.9 \\
\hline 1 & $13(50)$ & $15(44)$ & \\
\hline 2 & $6(23)$ & $10(29)$ & \\
\hline 3 & $7(27)$ & $9(27)$ & \\
\hline Clinical syndrome & & & 0.8 \\
\hline \multicolumn{4}{|l|}{ Stable angina CCS class } \\
\hline I & $8(31)$ & $9(27)$ & \\
\hline II & $11(42)$ & $18(53)$ & \\
\hline III & $5(19)$ & $4(12)$ & \\
\hline Unstable angina & $2(8)$ & $3(9)$ & \\
\hline \multicolumn{4}{|l|}{ Medication } \\
\hline Acetylsalicylic acid & $26(100)$ & $34(100)$ & 1.0 \\
\hline ACE inhibitors & $9(35)$ & $13(38)$ & 1.0 \\
\hline $\mathrm{AT}_{1}$ antagonists & $1(4)$ & $1(3)$ & 1.0 \\
\hline ß-Blockers & $16(62)$ & $20(59)$ & 1.0 \\
\hline Calcium channel blockers & $9(35)$ & $8(24)$ & 0.5 \\
\hline Diuretics & $9(35)$ & $9(26)$ & 0.7 \\
\hline Fibrates & $2(8)$ & $1(3)$ & 0.6 \\
\hline Insulin & $1(4)$ & $1(3)$ & 1.0 \\
\hline Nitrates & $13(50)$ & $23(68)$ & 0.3 \\
\hline Oral antidiabetics & $3(12)$ & $0(0)$ & 0.1 \\
\hline Statins & $16(62)$ & $33(97)$ & $<0.0005$ \\
\hline
\end{tabular}

Values are mean $\pm S D$ or $n(\%)$. CCS indicates Canadian Cardiovascular Society. 
Table 1 (part 2). Patient Demographics, Medications, and Laboratory Tests.

\begin{tabular}{lccc}
\hline & $\begin{array}{c}\text { Group A } \\
(\mathbf{n = 2 6 )}\end{array}$ & $\begin{array}{c}\text { Group B } \\
(\mathbf{n = 3 4 )}\end{array}$ & $\boldsymbol{P}$ \\
Laboratory tests* & & & \\
Total cholesterol, $\mathrm{mg} / \mathrm{dL}$ & $219 \pm 22$ & $173 \pm 29$ & $<0.0001$ \\
LDL cholesterol, mg/dL & $158 \pm 25$ & $89 \pm 20$ & $<0.0001$ \\
HDL cholesterol, mg/dL & $42 \pm 10$ & $51 \pm 14$ & $<0.01$ \\
Lipoprotein(a), mg/L & $32 \pm 30$ & $19 \pm 15$ & $<0.05$ \\
Triglycerides, $\mathrm{mg} / \mathrm{dL}$ & $114 \pm 76$ & $144 \pm 60$ & 0.1 \\
Apolipoprotein $\mathrm{A}_{1}, \mathrm{mg} / \mathrm{dL}$ & $149 \pm 20$ & $150 \pm 21$ & 0.8 \\
Apolipoprotein B, mg/dL & $116 \pm 16$ & $97 \pm 20$ & $<0.0005$ \\
Apolipoprotein B/A, ratio & $0.79 \pm 0.11$ & $0.65 \pm 0.13$ & $<0.0001$ \\
Fibrinogen, $\mathrm{mg} / \mathrm{dL}$ & $313 \pm 85$ & $272 \pm 71$ & $<0.05$ \\
\hline
\end{tabular}

* Mean values of measurements at time of initial and follow-up IVUS examinations. Values are mean $\pm S D$

\section{Baseline IVUS Data}

The baseline IVUS characteristics were similar between the 2 groups (Table 2). The majority of plaques showed a soft or fibrous composition.

Table 2. Baseline IVUS Data.

\begin{tabular}{lccc}
\hline & $\begin{array}{c}\text { Group A } \\
(\mathbf{n = 2 6 )}\end{array}$ & $\begin{array}{c}\text { Group B } \\
(\mathbf{n = 3 4 )}\end{array}$ & $\boldsymbol{P}$ \\
EEM CSA, $\mathrm{mm}^{2}$ & $25.1 \pm 6.1$ & $25.4 \pm 5.5$ & 0.9 \\
Lumen CSA, $\mathrm{mm}^{2}$ & $16.0 \pm 4.4$ & $15.4 \pm 4.2$ & 0.6 \\
P\&M CSA, mm & & $10.0 \pm 4.2$ & 0.4 \\
Plaque burden, \% & $9.1 \pm 3.1$ & $38.9 \pm 12.0$ & 0.3 \\
Total arc of calcium, degrees & $36.1 \pm 9.0$ & $66 \pm 105$ & 0.7 \\
Plaque composition, $\mathrm{n}(\%)$ & $79 \pm 111$ & & 0.9 \\
$\quad$ Soft & & $10(29)$ & \\
$\quad$ Fibrous & $6(23)$ & $11(32)$ & \\
$\quad$ Mixed & $9(35)$ & $3(9)$ & \\
$\quad$ Calcified & $2(8)$ & $10(29)$ & \\
\hline
\end{tabular}




\section{Serial IVUS Data}

Group A plaques (in patients with LDL cholesterol $\geq 120 \mathrm{mg} / \mathrm{dL}$ ) showed more P\&M progression than group B plaques $(24 / 26$ [92\%] versus $17 / 34$ [50\%], $P<0.001)$ and tended to have a greater frequency of lumen reduction (17/26 [65\%] versus 15/34 [44\%], $P=0.17)$. There was no significant difference in the incidence of EEM increase: $20 / 26(77 \%)$ versus $20 / 34(59 \%), P=N S$.

Absolute and relative annual changes of lumen, P\&M, and EEM CSA are presented in Table 3. There was no difference in annual changes of EEM size, but group A plaques had a greater annual increase in P\&M CSA $(P<0.0001)$ and a greater annual decrease in lumen CSA $(P<0.02$, Figure 1$)$. There was no change in IVUS plaque composition during follow-up or in total arc of calcium within the entire population or within groups $A$ and $B$ separately: $76 \pm 110^{\circ}, 80 \pm 114^{\circ}$, and $67 \pm 107^{\circ}$ ( $P>0.8$ versus baseline).

Table 3. Serial IVUS Data.

\begin{tabular}{lccc}
\hline & $\begin{array}{c}\text { Group A } \\
(\mathbf{n = 2 6 )}\end{array}$ & $\begin{array}{c}\text { Group B } \\
(\mathbf{n = 3 4 )}\end{array}$ & $\boldsymbol{P}$ \\
$\Delta$ EEM CSA/y, mm & & & \\
$\Delta$ EEM CSA/y, \% & $0.2 \pm 3.1$ & $0.6 \pm 4.0$ & 0.6 \\
$\Delta \mathrm{P} \& M$ CSA $/ y, \mathrm{~mm}^{2}$ & $2.3 \pm 11.0$ & $3.3 \pm 14.0$ & 0.8 \\
$\Delta \mathrm{P} \& M \mathrm{MSA} / \mathrm{y}, \%$ & $1.5 \pm 1.1$ & $0.2 \pm 1.5$ & $<0.0001$ \\
$\Delta$ Lumen CSA/y, $\mathrm{mm}^{2}$ & $21.2 \pm 18.1$ & $4.0 \pm 15.2$ & $<0.0005$ \\
$\Delta$ Lumen CSA/y, \% & $-1.4 \pm 2.8$ & $0.5 \pm 2.9$ & $<0.02$ \\
\hline
\end{tabular}




\section{$\Delta \mathrm{CSA} /$ year $\left(\mathrm{mm}^{2}\right)$}

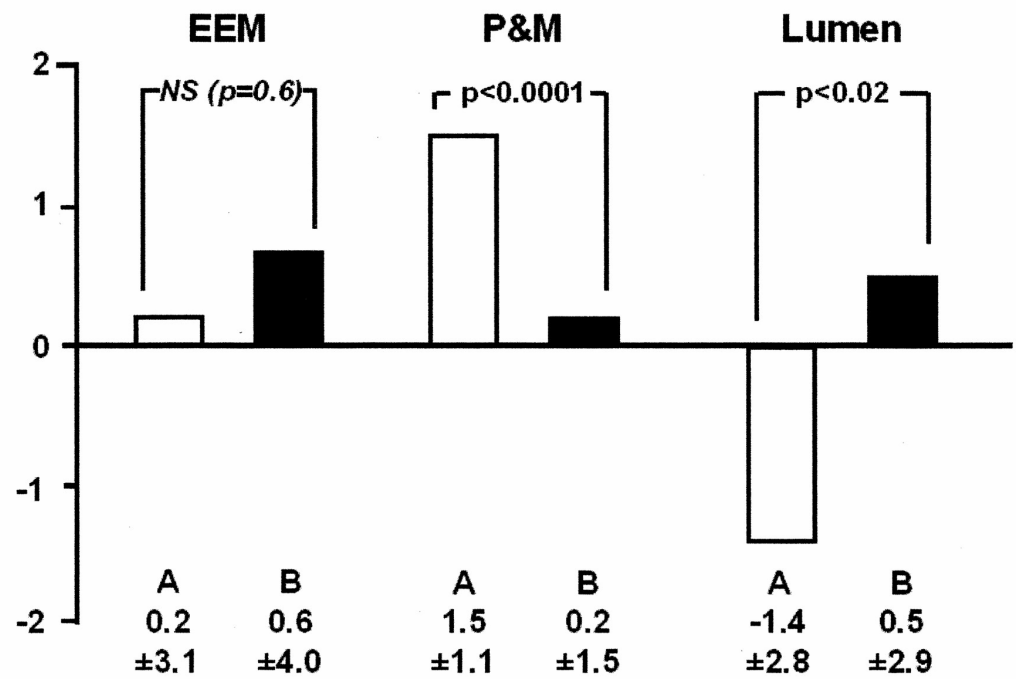

Figure 1. Annual changes of IVUS parameters in group A (LDL cholesterol $\geq 120 \mathrm{mg} / \mathrm{dL}$ ) vs group B (LDL cholesterol $<120 \mathrm{mg} / \mathrm{dL}$ ) plaques.

\section{Relation Between Cholesterol and Serial IVUS Data}

There was a positive linear relation between percent annual changes in P\&M CSA versus $\mathrm{LDL}$ cholesterol $(r=0.41, P<0.0001)$. There was a negative linear relation between percent annual changes in lumen size versus LDL cholesterol $(r=-0.32$, $P<0.01)$ (Figure 2). There was a negative linear relation between annual changes in P\&M CSA versus HDL cholesterol $(r=-0.30, P<0.02)$; this relation remained significant even after removal of the 2 outliers with $\mathrm{HDL}$ cholesterol $>80 \mathrm{mg} / \mathrm{dL}(r=-$ $0.36 ; y=-0.67 x+42.0 ; P<0.01 ; n=58)$. However, there was no relation between either LDL or HDL cholesterol and annual changes in EEM CSA. The LDL/HDL ratio showed relations similar to $L D L$ cholesterol alone (Figure 2).

The relation between annual changes in P\&M CSA and LDL cholesterol demonstrated that an LDL value of $75 \mathrm{mg} / \mathrm{dL}$ was the cutoff at which regression analysis predicted no average annual plaque increase (Figure 3). However, individual patients exhibited plaque increase even at lower LDL cholesterol values. Similarly, a value of the LDL/HDL ratio of 1.3 was the cutoff at which regression analysis predicted no average annual plaque increase. 

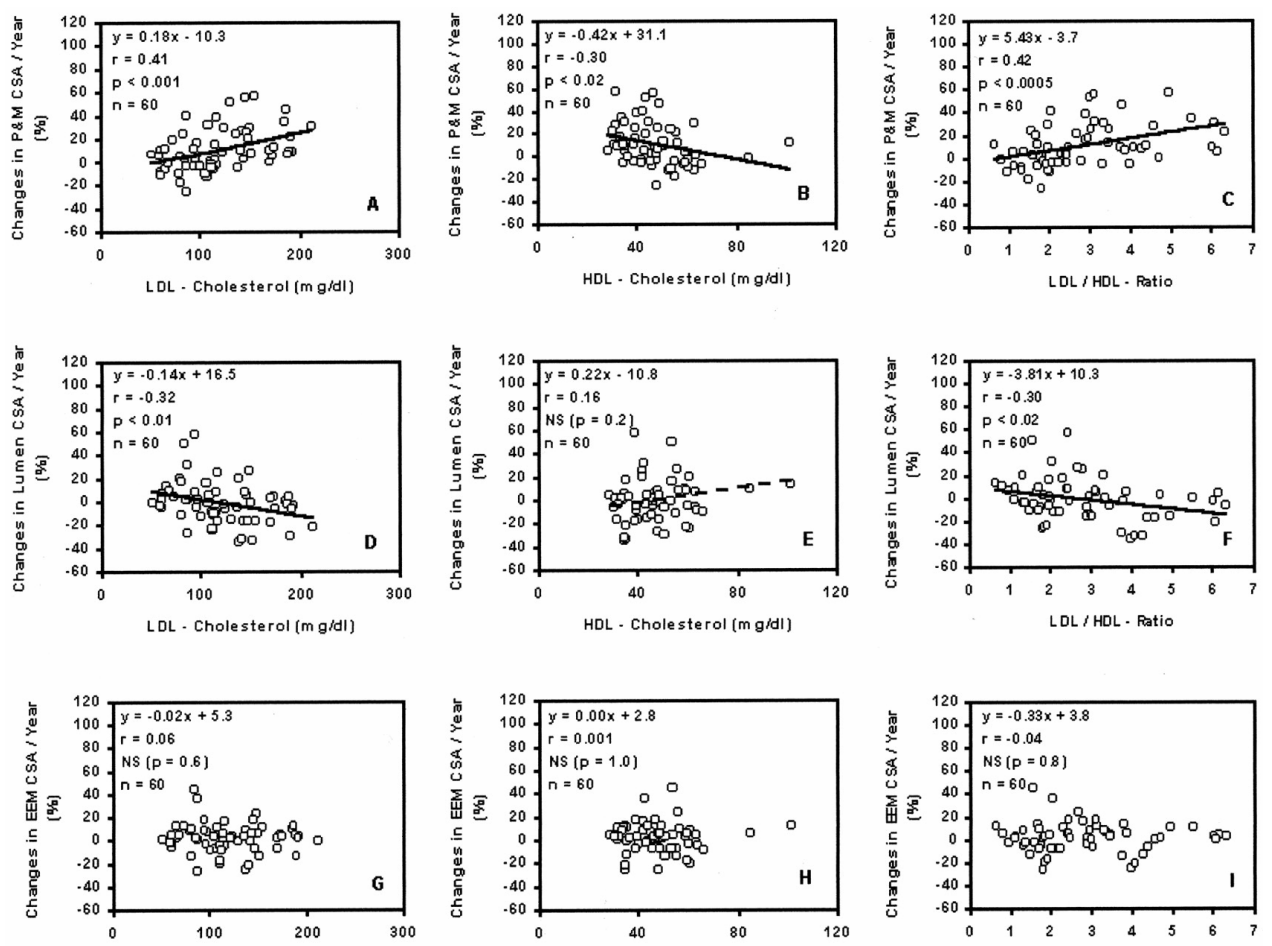

LDL - Chole sterol (m g/d)

HDL - Cholesterol (m gidl)

LDL / HDL. Ratio

Figure 2. Relation between LDL cholesterol (left), HDL cholesterol (middle), and LDL/HDL ratio (right) vs. serial IVUS data.

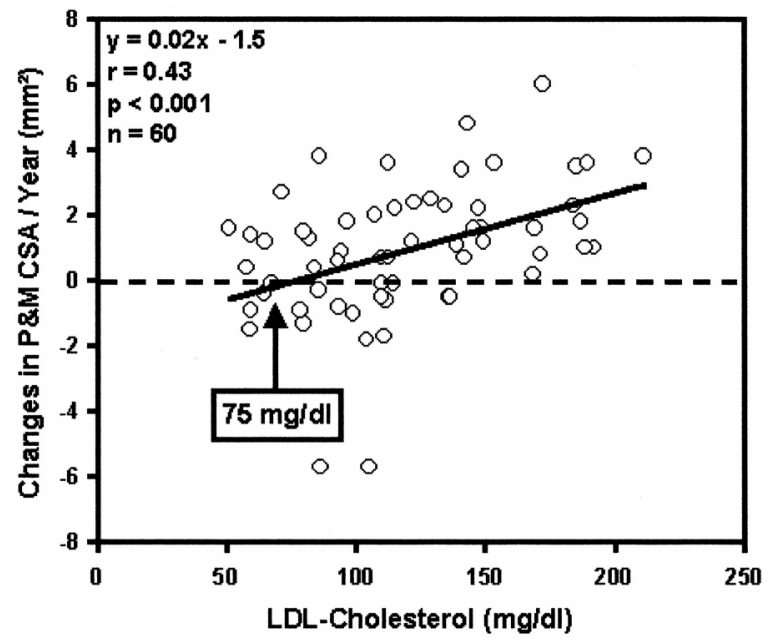

Figure 3. Relation between LDL cholesterol and annual changes in P\&M CSA. An LDL value of $75 \mathrm{mg} / \mathrm{dL}$ was the cutoff at which regression analysis predicts no average annual P\&M CSA increase. 


\section{Cholesterol and Serial IVUS Data in Patients Treated With Statins}

When only those patients who were on statins were analyzed $(n=49)$, there was still a significant positive linear relation between percent annual changes in P\&M CSA versus LDL cholesterol $(r=0.45, P<0.001)$ and a negative linear relation between annual changes in P\&M CSA versus HDL cholesterol $(r=-0.30, P<0.05)$. An LDL value of $72.5 \mathrm{mg} / \mathrm{dL}$ was the cutoff at which regression analysis predicted no average annual P\&M CSA increase. Percent annual changes in lumen CSA tended to show a negative linear relation with $L D L$ cholesterol $(r=-0.25, P=0.12)$, but there was no relation with $\mathrm{HDL}$ cholesterol $(r=0.14, \mathrm{y}=0.18 \mathrm{x}-6.6 ; P=0.35)$. Moreover, there was no relation between either LDL or HDL cholesterol and annual changes in EEM CSA ( $r=-0.02$ for both). The number of patients who were not on a statin $(n=11)$ was too small to permit similar meaningful analysis.

\section{Discussion}

We found (1) a positive linear relation between LDL cholesterol and annual changes in P\&M area with (2) an LDL value of $75 \mathrm{mg} / \mathrm{dL}$ as the cutoff at which regression analysis predicted, on average, no annual P\&M area increase; (3) an inverse relation between HDL cholesterol and annual changes in P\&M area; and (4) no relation between either LDL or HDL cholesterol and annual changes in total arterial area (ie, arterial remodeling). Because LDL and HDL cholesterol levels did not appear to affect arterial remodeling, there was an inverse relation between LDL cholesterol and annual changes in lumen area. Finally, although they had similar IVUS characteristics at baseline, patients with LDL cholesterol $\geq 120 \mathrm{mg} / \mathrm{dL}$ showed more annual plaque progression and lumen reduction than did patients with lower LDL cholesterol values.

\section{Serial Plaque and Lumen Changes and Cholesterol}

There is ample accumulated evidence of the significance of cholesterol on the progression of atherosclerosis. ${ }^{19-21}$ The present linear relations between LDL cholesterol and both P\&M progression and lumen reduction agree with previous studies, and they emphasize the importance of lowering total cholesterol and LDL cholesterol for preventing disease progression. ${ }^{1-4,22-24}$ The inverse relation between HDL cholesterol and IVUS P\&M progression in the present study is also in good agreement with previous studies ${ }^{25,26}$ that underlined the importance of increasing HDL cholesterol levels.

Previous large (nonserial) histopathological studies have demonstrated the relation between serum cholesterol and atherosclerotic disease severity ${ }^{27,28}$ and 
plaque vulnerability. ${ }^{29}$ Our study extends these previous observations by providing serial morphological evidence for the clinically established relation between disease progression and serum lipids.

Previous serial IVUS studies in native coronary arteries did not address the relation between cholesterol levels and plaque progression. These IVUS studies compared treatment with a particular statin versus dietary stabilization or usual care. $^{13-15}$

Cholesterol lowering is an accepted principle in reducing the risk of coronary artery disease, and the extent to which cholesterol is lowered appears to be important. In addition, a greater reduction of LDL cholesterol is associated with a greater reduction in the risk of cardiovascular events, but clinical studies have not determined definitively whether there is a benefit in lowering cholesterol to very low levels. ${ }^{30}$ Our present study suggests that an LDL cholesterol value of $75 \mathrm{mg} / \mathrm{dL}$ is on average associated with no plaque progression. Importantly, because ethnic factors may influence the response to serum cholesterol levels, ${ }^{31}$ the results of our study may apply only to white patients.

Our study did not address pharmacological intervention (lipid lowering) but rather was a clinical observational study in patients with coronary artery disease treated by conventional medical therapy, including statins in the vast majority of patients. The nature of our investigation implies that potential pleiotropic effects of statins could have contributed to our findings; ${ }^{32}$ however, the data are not currently available to permit further subanalyses to exclude the effect of statins. Nevertheless, when we analyzed only those patients who were on a statin, the relations between LDL cholesterol and HDL cholesterol versus the percent annual changes in plaque size remained unchanged.

Baseline total arterial CSA was identical in patients with higher $(\geq 120 \mathrm{mg} / \mathrm{dL})$ versus lower LDL cholesterol but was (for both groups) significantly larger than that of a historic population of nondiseased LM coronary arteries. ${ }^{11}$ This suggests that, at baseline, plaques in both groups were already accompanied by positive (compensatory) arterial remodeling. ${ }^{11,12}$ Moreover, both groups had a slight but similar further increase in total arterial area not related to LDL or HDL cholesterol levels. The variability of remodeling responses ${ }^{12}$ may partially explain progressive lumen narrowing in some (but not all) individuals despite an effective modification of the lipid profile. ${ }^{24}$ This is in contrast to one histopathological study showing a modest linear relationship between HDL cholesterol and positive remodeling assessed at a single time point. ${ }^{33}$

\section{Limitations}

Although by most standards, this was a large serial IVUS study, all studies with 
long-term serial assessment of atherosclerosis are limited to a relatively small number of patients. The data of this study are unique and may well reflect clinical reality. However, because retrospective analyses of prospectively acquired data (demographics, medication, and laboratory tests) were performed, we cannot rule out a certain selection bias; we were able to include only patients with significant coronary artery disease who were admitted for repeat cardiac catheterization $\geq 12$ months after baseline (this limitation applies to both groups). Therefore, the findings of the present study may not be applicable to the general population. We used 2 IVUS systems in the present study; however, when we compared the data from the 2 different IVUS systems, we found no differences, and separate linear regression analyses in data sets that were obtained by one or the other IVUS system provided almost identical results. Furthermore, individual patients were imaged with the same system at index and follow-up. 3D (ECG-gated) IVUS analysis $^{34}$ may be superior for the assessment of coronary dimensions and provides volumetric data. In addition, sophisticated computer-aided gray-scale IVUS analyses ${ }^{15}$ or radiofrequency IVUS analyses ${ }^{35}$ may be superior to visual IVUS analysis of plaque composition.

\section{Conclusions}

Our data demonstrate a positive linear relation between LDL cholesterol and annual changes in plaque size, with an LDL value of $75 \mathrm{mg} / \mathrm{dL}$ as cutoff level that, on average, predicts no plaque progression. In addition, HDL cholesterol reveals an inverse relation with annual changes in plaque size.

\section{References}

1. Scandinavian Simvastatin Survival Study Group. Randomized trial of cholesterol lowering in 4444 patients with coronary heart disease: the Scandinavian Simvastatin Survival Study (4S). Lancet 1994;344:1383-1389.

2. Sacks FM, Pfeffer MA, Moye LA, et al. for the Cholesterol And Recurrent Events (CARE) Trial Investigators. The effect of pravastatin on coronary events after myocardial infarction in patients with average cholesterol levels. N Engl J Med 1996;335:1001-1009.

3. Shepherd J, Cobbe SM, Ford I, et al. for the West of Scotland Coronary Prevention Study Group. Prevention of coronary artery disease with pravastatin in men with hypercholesterolemia. $\mathrm{N}$ Engl J Med 1995;333:1301-1307.

4. Downs JR, Clearfield M, Weis S, et al. Primary prevention of acute coronary events with lovastatin in men and women with average cholesterol levels: results of AFCAPS/TexCAPS. Air Force/Texas Coronary Atherosclerosis Prevention Study. JAMA 1998;279:1615-1622.

5. Devereux R, Alderman M. Role of preclinical cardiovascular disease in the evolution from risk factor exposure to development of morbid events. Circulation 1993;88:1444-1445.

6. Waters D, Craven TE, Lespérance J. Prognostic significance of progression of coronary atherosclerosis. Circulation 1993;87:1067-1075. 
7. Achenbach S, Ropers D, Pohle K, et al. Influence of lipid-lowering therapy on the progression of coronary artery calcification: a prospective evaluation. Circulation 2002;106:1077-1082.

8. Archbold RA, Timmis AD. Modification of coronary artery disease progression by cholesterollowering therapy: the angiographic studies. Curr Opin Lipidol 1999;10:527-534.

9. Mintz GS, Nissen SE, Anderson WD, et al. American College of Cardiology clinical expert consensus document on standards for acquisition, measurement and reporting of intravascular ultrasound studies (IVUS). J Am Coll Cardiol 2001;37:1478-1492.

10. von Birgelen C, Klinkhart W, Mintz GS, et al. Plaque distribution and vascular remodeling of ruptured and nonruptured coronary plaques in the same vessel: an intravascular ultrasound study in vivo. J Am Coll Cardiol 2001;37:1864-1870.

11. Ge J, Liu F, Görge G, et al. Angiographically "silent" plaque in the left main coronary artery detected by intravascular ultrasound. Coron Artery Dis 1995;6:805-810.

12. von Birgelen C, Airiian SG, Mintz GS, et al. Variations of remodeling in response to left main atherosclerosis assessed with intravascular ultrasound in vivo. Am J Cardiol 1997;80:1408-1413.

13. Takagi T, Yoshida K, Akasaka T, et al. Intravascular ultrasound analysis of reduction in progression of coronary narrowing by treatment with pravastatin. Am J Cardiol 1997;79:1673-1676.

14. Shiran A, Mintz GS, Leiboff B, et al. Serial volumetric intravascular ultrasound assessment of arterial remodeling in left main coronary artery disease. Am J Cardiol 1999;83:1427-1432.

15. Schartl M, Bocksch W, Koschyk DH, et al. for the GAIN-Study Investigators. Use of intravascular ultrasound to compare effects of different strategies of lipid-lowering therapy on plaque volume and composition in patients with coronary artery disease. Circulation 2001;104:387-392.

16. Bergelson BA, Tommaso CL. Left main coronary artery disease: assessment, diagnosis, and therapy. Am Heart J 1995;129:350-359.

17. Baumgart $\mathrm{D}$, Haude $\mathrm{M}, \mathrm{Ge} \mathrm{J}$, et al. Online integration of intravascular ultrasound images into angiographic images. Cathet Cardiovasc Diagn 1996;39:328-329.

18. Callister TQ, Raggi $P$, Cooil $B$, et al. Effect of HMG-CoA reductase inhibitors on coronary artery disease as assessed by electron-beam computed tomography. N Engl J Med 1998;339:1972-1978.

19. Kannel WB, Castelli WP, Gordon T. Cholesterol in the prediction of atherosclerotic disease: new perspectives based on the Framingham study. Ann Intern Med 1979;90:85-91.

20. Miettinen M, Turpeinen O, Karvonen MJ, et al. Effect of cholesterol-lowering diet on mortality from coronary heart-disease and other causes: a twelve-year clinical trial in men and women. Lancet 1972;2:835-838.

21. Stamler J, Wentworth D, Neaton JD. Is relationship between serum cholesterol and risk of premature death from coronary heart disease continuous and graded? Findings in 356,222 primary screenees of the Multiple Risk Factor Intervention Trial (MRFIT). JAMA 1986;256:2823-2828.

22. The Lipid Research Clinics Coronary Primary Prevention Trial results, II: the relationship of reduction of coronary heart disease to cholesterol lowering. JAMA 1984;251:365-374.

23. Verschuren WM, Jacobs DR, Bloemberg BP, et al. Serum total cholesterol and long-term coronary heart disease mortality in different cultures: twenty-five-year follow-up in the Seven Countries Study. JAMA 1995;274:131-136.

24. McKenney J. Combination therapy for elevated low-density lipoprotein cholesterol: the key to coronary artery disease risk reduction. Am J Cardiol 2002;90(suppl):8K-20K.

25. Miller NE, Thelle DS, Forde $\mathrm{OH}$, et al. The Tromso heart-study. High-density lipoprotein and coronary heart-disease: a prospective case-control study. Lancet 1977;1:965-968.

26. Rubins HB, Robins SJ, Collins D, et al. Gemfibrozil for the secondary prevention of coronary heart disease in men with low levels of high-density lipoprotein cholesterol. Veterans Affairs HighDensity Lipoprotein Cholesterol Intervention Trial Study Group. N Engl J Med 1999;341:410-418.

27. Newman WP III, Freedman DS, Voors AW, et al. Relation of serum lipoprotein levels and systolic blood pressure to early atherosclerosis: the Bogalusa Heart Study. N Engl J Med 1986;314:138144. 
28. McGill HC Jr, McMahan CA, Malcom GT, et al, the Pathobiological Determinants of Atherosclerosis in Youth (PDAY) Research Group. Effects of serum lipoproteins and smoking on atherosclerosis in young men and women. Arterioscler Thromb Vasc Biol 1997;17:95-106.

29. Burke AP, Farb A, Malcom GT, et al. Coronary risk factors and plaque morphology in men with coronary disease who died suddenly. N Engl J Med 1997;336:1276-1282.

30. Kastelein JJP. The future of best practice. Atherosclerosis 1999;143(suppl1):S17-S21.

31. Varghese PJ, Arumugam SB, Cherian KM, et al. Atheromatous plaque reflects serum total cholesterol levels: a comparative morphologic study of endarterectomy coronary atherosclerotic plaques removed from patients from the southern part of India and Caucasians from Ottawa, Canada. Clin Cardiol 1998;21:335-340.

32. Evans $M$, Roberts A, Rees A. The future direction of cholesterol-lowering therapy. Curr Opin Lipidol 2002;13:663-669.

33. Taylor AJ, Burke AP, Farb A, et al. Arterial remodeling in the left coronary system: the role of highdensity lipoprotein cholesterol. J Am Coll Cardiol 1999;34:760-767.

34. von Birgelen C, de Vrey EA, Mintz GS, et al. ECG-gated three-dimensional intravascular ultrasound: feasibility and reproducibility of the automated analysis of coronary lumen and atherosclerotic plaque dimensions in humans. Circulation 1997;96:2944-2952.

35. Nair A, Kuban BD, Tuzcu EM, et al. Coronary plaque classification with intravascular ultrasound radiofrequency data analysis. Circulation 2002;106:2200-2206. 


\section{Chapter 3}

Relation Between Plaque Progression and Low-Density Lipoprotein Cholesterol During Aging as Assessed With Serial Long-Term ( $\geq 12$ Months)

Intravascular Ultrasound of the Left Main Coronary Artery

Marc Hartmann, Clemens von Birgelen, Gary S. Mintz, Gert K. van Houwelingen, Holger Eggebrecht, Dirk Böse, Heinrich Wieneke, Patrick M.J. Verhorst, Raimund Erbel

Reprinted with permission from:

The American Journal of Cardiology 2006;98(11):1419-1423.

(C) 2006 Elsevier Inc. 



\section{Abstract}

Because of the clinical benefit of lipid lowering in older patients, we hypothesized that the relation between low-density lipoprotein (LDL) cholesterol serum levels and coronary plaque progression may persist throughout aging.

We analyzed serial intravascular ultrasound (IVUS) data of 60 left main stems $(18 \pm 9$ months apart) and evaluated the relation between LDL cholesterol levels and coronary plaque progression at different ages. The population $(n=60)$ was divided into 3 groups according to age: tertile $1(n=20)$ was a mean age of $48 \pm 6$ years (median 51 , range 33 to 55$)$, tertile $2(n=20)$ was a mean age of $58 \pm 2$ years (median 59 , range 55 to 61 ), and tertile $3(n=20)$ was a mean age of $66 \pm 6$ years (median 65 , range 61 to 83 ). Between groups, there was no significant difference in non-age-related demographics, clinical data, lipid profiles, or medications (e.g., statins). There was a positive linear relation between LDL cholesterol and annual changes in plaque plus media area in all age tertiles, which was statistically significant in tertiles 2 and $3(r=0.56, p<0.01 ; r=0.50$, $p<0.02)$ and showed a strong trend in tertile $1(r=0.41, p=0.07)$. The estimated LDL cholesterol thresholds, which, as determined by regression analysis, would correspond to no plaque progression, were 74,60 , and $78 \mathrm{mg} / \mathrm{dl}$, respectively, in tertiles 1,2 , and 3 .

In conclusion, serial IVUS data in left main coronary arteries suggest that the relation between LDL cholesterol serum levels and plaque progression persists during aging.

\section{Introduction}

In many countries with a Western lifestyle, the population is aging, and age is a well-known risk factor of cardiovascular events. ${ }^{1-8}$ Up to now, there have been no data on the relation between low-density lipoprotein (LDL) cholesterol serum levels and coronary plaque progression at different stages of aging. Because of the beneficial clinical effect of lipid lowering, ${ }^{4-12}$ we hypothesized that the relation between LDL cholesterol serum levels and plaque progression should persist during aging. To investigate this hypothesis, we reanalyzed previously reported clinical and serial intravascular ultrasound (IVUS) data of atherosclerotic left main (LM) coronary arteries in 60 patients with established coronary artery disease. ${ }^{13-15}$

\section{Methods}

\section{Study population}

We reanalyzed serial IVUS data in age tertiles from a previously reported population of 60 patients who had hemodynamically nonsignificant de novo LM atherosclerotic lesions. ${ }^{13-15}$ All patients met the following criteria: (1) serial highquality IVUS imaging of the entire LM stem $\geq 12$ months apart; (2) calcium deposits that did not limit the quantitative assessment of vessel area (shadowing $\leq 75^{\circ}$ of the adventitial circumference by individual calcium deposit); (3) nonostial target site 
location; (4) angiographic lumen diameter stenosis <30\% ("worst-view" visual assessment); and (5) no intervention in the very proximal left anterior descending or circumflex coronary artery segments because these interventions could have affected the LM artery. Patients were examined in the Essen University Cardiac Catheterization Laboratory (Essen, Germany) with a follow-up of $18 \pm 9$ months. The IVUS study was approved by the local council on human research, and all patients signed a written informed consent form as approved by the local medical ethics committee.

\section{Demographics, medication, and lipid profile}

Demographics, cardiovascular risk factors, medication, and lipid profiles were prospectively recorded in our laboratory, including diabetes mellitus and hypertension (medication dependent), hypercholesterolemia (medication dependent, total serum cholesterol $>200 \mathrm{mg} / \mathrm{dl}$ or LDL cholesterol $>160 \mathrm{mg} / \mathrm{dl}$ ), history of smoking, and family history of coronary artery disease. Data of laboratory tests were the mean of baseline and follow-up values. Plasma concentrations of total cholesterol, LDL cholesterol, high-density lipoprotein cholesterol, and triglycerides were measured by standard enzymatic methods. Medication was recorded only if drugs were taken for $>50 \%$ of the follow-up interval (e.g., clopidogrel for 4 weeks was not tabulated).

\section{IVUS imaging}

IVUS was performed as previously described. ${ }^{13,16}$ In brief, IVUS studies were performed during percutaneous coronary interventions of mid or distal left anterior or left circumflex arteries after intracoronary injections of $200 \mu \mathrm{g}$ of nitroglycerin. Two commercial systems were used: a mechanical sector scanner (Boston Scientific Corporation, San Jose, California) incorporating a 30-MHz single-element beveled transducer or a solid-state device (Endosonics, Rancho Cordova, California). Importantly, as is standard procedure at Essen University, if a patient underwent imaging with 1 IVUS system during an index procedure, the same IVUS system was used at follow-up. Slow continuous pullbacks of the IVUS transducer were started as distal as possible in 1 of the left coronary arteries and were generally performed using a motorized pull-back device (at $0.5 \mathrm{~mm} / \mathrm{s}$ ). IVUS images of the entire pullback were recorded on 0.5-in high-resolution s-VHS tape for off-line analysis. In addition, a dedicated image-in-image system (Echo-Map, Siemens, Erlangen, Germany) $)^{17}$ was used to record the "angiographic" position of the IVUS probe together with the corresponding IVUS image, especially at sites of characteristic landmarks (i.e., calcifications or unusual plaque shapes) and/or the 
target site. Follow-up IVUS studies were performed using the same IVUS system as used initially.

\section{IVUS analysis}

IVUS analysis was performed as previously described. ${ }^{13}$ In brief, the target lesion site image slice was the slice with the smallest lumen area. Exact matching of the initial and follow-up IVUS studies was ensured using side-by-side comparison of the serial IVUS video sequences and information of the pull-back speed, ${ }^{13,16}$ the operators' recorded comments (on videotape), characteristic calcifications, vascular and perivascular landmarks, and plaque shapes. If required, the angiographic sequences of the dedicated image-in-image system (Echo-Map) were revisited to optimize matching. ${ }^{13,17}$ The lumen area was measured by tracing the leading edge of the intima. The total vessel area was measured by tracing the leading edge of the adventitia. As in many previous IVUS studies, plaque plus media was used as a measurement of atherosclerotic plaque because IVUS cannot measure media thickness accurately. ${ }^{16}$ Plaque burden (percentage) was calculated as (plaque $\&$ media divided by vessel cross-sectional area) $\times 100 \%$. Predominant plaque composition (soft, fibrous, calcified, or mixed) was assessed visually as previously described. ${ }^{13,16}$ The arc of calcium was measured with a protractor centered on the lumen; if necessary, the total arc of calcium was obtained by adding arcs of individual deposits. Plaques were classified as calcified if the total arc of lesion calcium was $>180^{\circ}$. Extrapolation of the total vessel area boundary behind calcium was possible if each patient's calcific deposit did not shadow $>75^{\circ}$ of the adventitial border. We calculated the changes between initial and follow-up IVUS measurements. To compensate for the variation in follow-up intervals and to obtain comparable data, measurements were normalized for the length of the follow-up period, and annual changes (i.e., changes per year) were reported and compared. We created age tertiles to compare demographics, medication, lipid profile, IVUS data, and the relation between LDL cholesterol and changes in plaque plus media area.

\section{Statistical analysis}

Analyses were performed with SPSS 13.0 (SPSS Inc., Chicago, Illinois). Dichotomous data are presented as frequencies and compared using chi-square statistics or Fisher's exact test. Quantitative data are presented as mean \pm 1 SD and were compared using linear regression analysis or analysis of variance for repeated measurements with post hoc testing with the Tukey honestly significant difference test. A $p$ value $<0.05$ was considered statistically significant. 


\section{Results}

Demographics, medication, and lipid profile

The mean age of the entire population $(n=60)$ was $58 \pm 8$ years (median 59 , range 33 to 83$)$. Patients were categorized into tertiles $(n=20)$ according to age: tertile 1 (median age 51 years, range 33 to 55), tertile 2 (median age 59 years, range 55 to 61 ), and tertile 3 (median age 65, range 61 to 83). Across these 3 groups, there was no significant difference in demographics (except age), medication (e.g., statin use), and lipid profiles (Table 1).

Table 1 (part 1). Demographics, medication, and lipid profile of age tertiles

\begin{tabular}{lrrrr}
\hline Variable & $\begin{array}{r}\text { Tertile 1 } \\
(\mathbf{n = 2 0 )}\end{array}$ & $\begin{array}{r}\text { Tertile 2 } \\
\mathbf{( n = 2 0 )}\end{array}$ & $\begin{array}{r}\text { Tertile 3 } \\
(\mathbf{n = 2 0})\end{array}$ & p Value \\
Age (yrs) & $48 \pm 6$ & $58 \pm 2$ & $66 \pm 6$ & $<0.0001^{*}$ \\
Time of follow-up (mo) & $16 \pm 6$ & $20 \pm 11$ & $20 \pm 11$ & 0.3 \\
Men & $19(95 \%)$ & $17(85 \%)$ & $14(70 \%)$ & 0.1 \\
Body mass index (kg/m $\left.{ }^{2}\right)$ & $26 \pm 4$ & $26 \pm 2$ & $27 \pm 4$ & 0.5 \\
Hypercholesterolemia $\mp$ & $10(50 \%)$ & $8(40 \%)$ & $10(50 \%)$ & 0.8 \\
Systemic arterial hypertension & $13(66 \%)$ & $15(75 \%)$ & $15(75 \%)$ & 0.7 \\
Diabetes mellitus & $3(15 \%)$ & $2(10 \%)$ & $3(15 \%)$ & 0.8 \\
Smoker & $8(40 \%)$ & $3(15 \%)$ & $5(25 \%)$ & 0.2 \\
Previous myocardial infarction & $5(25 \%)$ & $6(30 \%)$ & $10(50 \%)$ & 0.2 \\
No. of coronary arteries narrowed $>50 \%$ & & & & \\
1 & $11(55 \%)$ & $9(45 \%)$ & $8(40 \%)$ & 0.6 \\
2 & $4(20 \%)$ & $7(35 \%)$ & $5(25 \%)$ & 0.6 \\
3 & $5(25 \%)$ & $4(20 \%)$ & $7(35 \%)$ & 0.5 \\
\hline
\end{tabular}

Post hoc testing: $p<0.01$ for tertile 1 versus 2, tertile 1 versus 3, and tertile 2 versus 3 . $\mp$ Medication dependent, total serum cholesterol >200 mg/dl, or LDL cholesterol >160 mg/dl. 
Table 1 (part 2). Demographics, medication, and lipid profile of age tertiles

\begin{tabular}{|c|c|c|c|c|}
\hline Variable & $\begin{array}{l}\text { Tertile } 1 \\
(n=20)\end{array}$ & $\begin{array}{l}\text { Tertile } 2 \\
(n=20)\end{array}$ & $\begin{array}{l}\text { Tertile } 3 \\
(n=20)\end{array}$ & p Value \\
\hline \multicolumn{5}{|l|}{ CCS class of stable angina pectoris } \\
\hline I & $6(30 \%)$ & $5(25 \%)$ & $6(30 \%)$ & 0.9 \\
\hline II & $11(55 \%)$ & $10(50 \%)$ & $8(40 \%)$ & 0.6 \\
\hline III & $1(5 \%)$ & $4(20 \%)$ & $4(20 \%)$ & 0.4 \\
\hline Unstable angina pectoris & $2(10 \%)$ & $1(5 \%)$ & $2(10 \%)$ & 0.7 \\
\hline \multicolumn{5}{|l|}{ Medication } \\
\hline Acetylsalicylic acid & $20(100 \%)$ & $20(100 \%)$ & $20(100 \%)$ & 1.0 \\
\hline Angiotensin-converting enzyme inhibitors & $7(35 \%)$ & $8(40 \%)$ & $7(35 \%)$ & 0.9 \\
\hline Angiotensin receptor antagonists & $1(5 \%)$ & $0(0 \%)$ & $1(5 \%)$ & 0.6 \\
\hline$\beta$ blockers & $12(60 \%)$ & $11(55 \%)$ & $13(65 \%)$ & 0.7 \\
\hline Calcium channel blockers & $5(25 \%)$ & $5(25 \%)$ & $7(35 \%)$ & 0.7 \\
\hline Diuretics & $3(15 \%)$ & $6(30 \%)$ & $9(45 \%)$ & 0.1 \\
\hline Fibrates & $2(10 \%)$ & $0(0 \%)$ & $1(5 \%)$ & 0.2 \\
\hline Insulin & $0(0 \%)$ & $1(5 \%)$ & $1(5 \%)$ & 0.6 \\
\hline Nitrates & $10(50 \%)$ & $12(60 \%)$ & $14(70 \%)$ & 0.4 \\
\hline Oral antidiabetics & $2(10 \%)$ & $1(5 \%)$ & $0(0 \%)$ & 0.2 \\
\hline Statins & $18(90 \%)$ & $17(85 \%)$ & $14(70 \%)$ & 0.2 \\
\hline \multicolumn{5}{|l|}{ Lipid profile } \\
\hline Total cholesterol (mg/dl) & $198 \pm 39$ & $193 \pm 48$ & $208 \pm 34$ & 0.3 \\
\hline LDL cholesterol (mg/dl) & $128 \pm 42$ & $118 \pm 52$ & $136 \pm 43$ & 0.6 \\
\hline High-density lipoprotein cholesterol (mg/dl) & $41 \pm 12$ & $49 \pm 13$ & $53 \pm 16$ & 0.2 \\
\hline Triglycerides (mg/dl) & $127 \pm 65$ & $147 \pm 84$ & $100 \pm 66$ & 0.3 \\
\hline
\end{tabular}

CCS = Canadian Cardiovascular Society . 
Baseline IVUS data

Baseline target lesion IVUS area measurements, percent plaque burden, visually assessed plaque composition (predominantly soft and fibrous), and total arc of calcium did not differ significantly across age tertiles (Table 2).

\section{Serial IVUS data}

Across age tertiles, there was no significant difference in annual changes in plaque plus media, lumen, and total vessel dimensions. The frequency of plaque plus media, lumen, and total vessel area increase or decrease also did not differ across tertiles (Table 2). For all patients, there was no relation between age and annual changes in plaque plus media $(r=0.02, p=0.9)$, lumen $(r=0.06, p=0.7)$, and total vessel area $(r=0.07, p=0.6)$. In all 3 age tertiles, IVUS plaque composition did not change significantly during follow-up, and there was no significant change in baseline total arc of calcium $\left(72^{\circ} \pm 109^{\circ}, 79^{\circ} \pm 87^{\circ}\right.$, and $76^{\circ} \pm 101^{\circ}$, respectively; $p>0.6$ ).

Table 2 (part 1). Baseline and serial intravascular ultrasound data of age tertiles.

\begin{tabular}{lcccc}
\hline Variable & $\begin{array}{c}\text { Tertile 1 } \\
(\mathbf{n = 2 0})\end{array}$ & $\begin{array}{c}\text { Tertile 2 } \\
(\mathbf{n}=20)\end{array}$ & $\begin{array}{c}\text { Tertile 3 } \\
(\mathbf{n}=20)\end{array}$ & p Value \\
Baseline lesion & $26.4 \pm 4.7$ & $23.4 \pm 4.4$ & $24.9 \pm 6.7$ & 0.2 \\
Total vessel area $\left(\mathrm{mm}^{2}\right)$ & $16.6 \pm 3.4$ & $14.3 \pm 3.1$ & $15.4 \pm 4.9$ & 0.2 \\
Lumen area $\left(\mathrm{mm}^{2}\right)$ & $9.8 \pm 4.7$ & $9.1 \pm 2.8$ & $9.6 \pm 3.4$ & 0.7 \\
Plaque \& media area $\left(\mathrm{mm}^{2}\right)$ & $36.0 \pm 13.2$ & $38.7 \pm 8.9$ & $38.5 \pm 9.5$ & 0.7 \\
Plaque burden $(\%)$ & $70 \pm 100$ & $78 \pm 83$ & $75 \pm 98$ & 0.6 \\
Total arc of calcium $\left(^{\circ}\right)$ & & & $4(20 \%)$ & 0.3 \\
Lesion plaque composition & $8(40 \%)$ & $4(20 \%)$ & $4(25 \%)$ & 0.2 \\
Soft & $5(25 \%)$ & $10(50 \%)$ & $5(25 \%)$ & 0.6 \\
Fibrous & $6(30 \%)$ & $5(25 \%)$ & $8(40 \%)$ & 0.5 \\
\hline Calcified & $1(5 \%)$ & $1(5 \%)$ & $3(15 \%)$ & \\
\hline Mixed & & &
\end{tabular}


Table 2 (part 2). Baseline and serial intravascular ultrasound data of age tertiles.

\begin{tabular}{lcccc}
\hline Variable & $\begin{array}{c}\text { Tertile 1 } \\
(\mathbf{n}=\mathbf{2 0})\end{array}$ & $\begin{array}{c}\text { Tertile 2 } \\
(\mathbf{n}=\mathbf{2 0})\end{array}$ & $\begin{array}{c}\text { Tertile 3 } \\
(\mathbf{n}=\mathbf{2 0})\end{array}$ & p Value \\
Lesion changes & $-0.4 \pm 4.4$ & $1.3 \pm 2.9$ & $1.1 \pm 3.5$ & 0.3 \\
Total vessel area/yr $\left(\mathrm{mm}^{2}\right)$ & $-0.6 \pm 14.6$ & $5.9 \pm 13.5$ & $5.0 \pm 14.1$ & 0.3 \\
Total vessel area/yr $(\%)$ & $-1.0 \pm 3.5$ & $-0.1 \pm 2.4$ & $0.0 \pm 3.0$ & 0.5 \\
Lumen area/yr $\left(\mathrm{mm}^{2}\right)$ & $-4.2 \pm 23.2$ & $0.5 \pm 17.7$ & $1.2 \pm 20.3$ & 0.7 \\
Lumen area/yr $(\%)$ & $0.6 \pm 2.5$ & $1.3 \pm 1.9$ & $1.2 \pm 1.8$ & 0.4 \\
Plaque \& media area/yr $\left(\mathrm{mm}^{2}\right)$ & $12.1 \pm 22.7$ & $19.3 \pm 30.5$ & $13.5 \pm 20.3$ & 0.2 \\
Plaque \& media area/yr $(\%)$ & & & & \\
Frequency of area changes & $13(65 \%)$ & $13(65 \%)$ & $14(70 \%)$ & 0.9 \\
Total vessel area/yr $>0$ & $7(35 \%)$ & $7(35 \%)$ & $6(30 \%)$ & \\
Total vessel area/yr $\leq 0$ & $6(30 \%)$ & $10(50 \%)$ & $12(60 \%)$ & 0.1 \\
Lumen area/yr $>0$ & $14(70 \%)$ & $10(50 \%)$ & $8(40 \%)$ & \\
\hline Lumen area/yr $\leq 0$ & $14(70 \%)$ & $14(70 \%)$ & $13(65 \%)$ & 0.9 \\
Plaque \& media area/yr $>0$ & $6(30 \%)$ & $6(30 \%)$ & $7(35 \%)$ & \\
Plaque \& media area/yr $\leq 0$ & & & & \\
\hline
\end{tabular}

Relation between plaque progression and LDL cholesterol in age tertiles

There was a positive linear relation between LDL cholesterol and annual changes in plaque plus media area in all age tertiles, which was statistically significance in tertiles 2 and $3(r=0.56, p<0.01 ; r=0.50, p<0.02)$ and showed a strong trend in tertile $1(r=0.41, p=0.07$; Figure 1). The estimated LDL cholesterol thresholds, which, by regression analysis, predicted no plaque progression, were 74,60 , and $78 \mathrm{mg} / \mathrm{dl}$, respectively, in tertiles 1,2 , and 3 . 

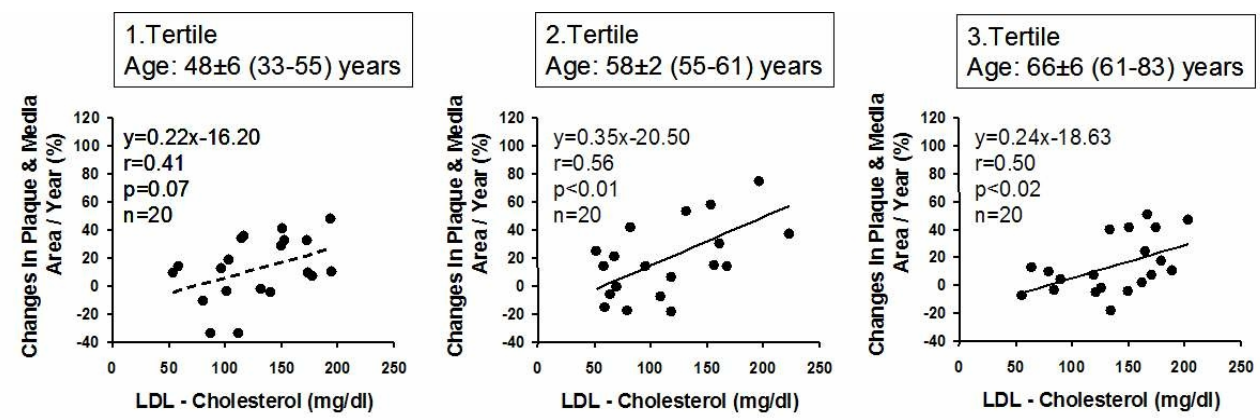

Figure 1. Relation between LDL cholesterol and plaque progression in age tertiles.

\section{Discussion}

The decrease in cardiovascular mortality associated with lowering LDL cholesterol has been attributed to a decrease in coronary plaque formation and progression and to stabilization of coronary plaques. ${ }^{18-20}$ Because of its high precision and reproducibility, IVUS is an ideal tool for serial assessment of plaque progression. ${ }^{16,21}$ As a result, IVUS allows conduction of short-duration studies with a relatively small sample compared with trials with clinical end points. ${ }^{22-24}$ Notably, IVUS-assessed coronary plaque progression has been linked with cardiovascular risk and adverse cardiovascular events. ${ }^{14}$

We recently reported a significant linear relation between serum LDL cholesterol levels and LM coronary plaque progression as assessed with serial IVUS imaging. ${ }^{13}$ Other serial IVUS studies have confirmed the presence of a linear relation between LDL cholesterol levels and extent of coronary plaque progression. ${ }^{22-24}$

Because age is a well-known cardiovascular risk factor, the relation between LDL cholesterol levels and cardiovascular events may become increasingly important during aging. ${ }^{1-8} \mathrm{~A}$ study with pravastatin administered to elderly patients at risk demonstrated a significant decrease in cardiovascular event rates that was attributed to lowering of LDL in older high-risk patients. ${ }^{4}$ Previous clinical trials that included older patients have shown that a certain decrease in LDL cholesterol results in similar risk decreases in younger and older patients. ${ }^{5,6}$ The findings of our present IVUS analysis add to the current knowledge by demonstrating that the relation between LDL cholesterol serum levels and LM coronary artery plaque progression persists during aging. The estimated LDL cholesterol threshold that predicted no plaque progression was $<80 \mathrm{mg} / \mathrm{dl}$ for all 3 
patient age groups.

Our findings underline previous clinical evidence of a comparable risk decrease in younger and older patients as a result of pharmacologic interventions to lower LDL cholesterol. ${ }^{5,6}$ These findings suggest a potential benefit of intensive LDL cholesterol lowering even in older patients. ${ }^{25,26}$ This concept is supported by findings of a subgroup analysis of the Reversal of Atherosclerosis with Aggressive Lipid Lowering (REVERSAL) trial, which showed significant plaque progression in patients with moderate LDL cholesterol lowering (to $110 \mathrm{mg} / \mathrm{dl}$ at follow-up), particularly in older patients. ${ }^{22}$

By most standards, this was a large serial IVUS study; however, all long-term serial IVUS studies are limited to a relatively small number of patients. We included patients who underwent non-LM intervention and who were admitted for repeat cardiac catheterization; thus, the findings of the present study may not be applicable to the general population. However, our observational study included patients with established coronary artery disease treated by conventional medical therapy, which may reflect clinical reality. Our retrospective analysis contains only a small number of patients $>75$ years of age; therefore, the findings may not be applicable to very old patients. We studied moderate LM disease as representative of nonintervened coronary segments; therefore, our findings may not be applicable to all (stenotic and nonstenotic) coronary segments.

IVUS radiofrequency analysis may be superior to quantify changes of plaque composition (e.g., plaque stabilization). ${ }^{27}$ We used 2 IVUS systems; although this approach may have shortcomings, every effort was taken to obtain the most reliable data possible, as previously discussed. ${ }^{13-16}$ Three-dimensional IVUS analysis may be superior to the 2-dimensional analysis approach that was used in the present study. ${ }^{23,28}$

\section{References}

1. Curb JD, Abbott RD, Rodriguez $\mathrm{BL}$, et al. Prospective association between low and high total lipoprotein cholesterol and coronary heart disease in elderly men. J Am Geriatr Soc 2004;52:19751980.

2. Lloyd-Jones DM, Wilson PWF, Larson MG, et al. Lifetime risk of coronary heart disease by cholesterol levels at selected ages. Arch Intern Med 2003;163:1966-1972.

3. Heart Protection Study Collaborative Group. MRC/BHF Heart Protection Study of cholesterol lowering with simvastatin in 20,536 high-risk individuals: a randomised placebo-controlled trial. Lancet 2002;360:7-22.

4. Shepherd J, Blauw GJ, Murphy MB, et al. Pravastatin in elderly individuals at risk of vascular disease (PROSPER): a randomised controlled trial PROspective Study of Pravastatin in the Elderly at Risk. Lancet 2002;360:1623-1630. 
5. Hunt D, Young P, Simes J, et al. Benefits of pravastatin on cardiovascular events and mortality in older patients with coronary heart disease are equal to or exceed those seen in younger patients: results from the LIPID trial. Ann Intern Med 2001;134:931-940.

6. Collins R, Armitage J. High-risk elderly patients PROSPER from cholesterol-lowering therapy. Lancet 2002;360:1618-1619.

7. Grundy SM, Cleeman JI, Merz CNB, et al. Implications of recent clinical trials for the National Cholesterol Education Program Adult Treatment Panel III Guidelines. J Am Coll Cardiol 2004:44:720-732.

8. Williams MA, Fleg JL, Ades PA, et al. Secondary prevention of coronary heart disease in the elderly (with emphasis on patients $>75$ years of age): an american heart association scientific statement from the council on clinical cardiology subcommittee on exercise, cardiac rehabilitation, and prevention. Circulation 2002;105:1735-1743.

9. Downs JR, Clearfield M, Weis S, et al. Primary prevention of acute coronary events with lovastatin in men and women with average cholesterol levels: results of AFCAPS/TexCAPS Airforce/Texas Coronary Atherosclerosis Prevention Study. JAMA 1998;279:1615-1622.

10. Shepherd J, Cobbe SM, Ford I, et al. Packard and West of Scotland Coronary Prevention Study Group: Prevention of coronary heart disease with pravastatin in men with hypercholesterolemia. N Engl J Med 1995;333:1301-1307

11. Sacks FM, Pfeffer MA, Moye LA, et al. and Cholesterol and Recurrent Events Trial investigators, The effect of pravastatin on coronary events after myocardial infarction in patients with average cholesterol levels. N Engl J Med 1996;335:1001-1009.

12. Scandinavian Simvastatin Survival Study Group. Randomised trial of cholesterol lowering in 4444 patients with coronary heart disease: the Scandinavian Simvastatin Survival Study (4S). Lancet 1994;344:1383-1389.

13. von Birgelen C, Hartmann M, Mintz GS, et al. Relation between progression and regression of atherosclerotic left main coronary artery disease and serum cholesterol levels as assessed with serial long-term ( $\geq 12$ months) follow-up intravascular ultrasound. Circulation 2003;108:2757-2762.

14. von Birgelen C, Hartmann M, Mintz GS, et al. Relationship between cardiovascular risk as predicted by established risk scores versus plaque progression as measured by serial intravascular ultrasound in left main coronary arteries. Circulation 2004;110:1579-1585.

15. von Birgelen C, Hartmann M, Mintz GS, et al. Remodeling index compared to actual vascular remodeling in atherosclerotic left main coronary arteries as assessed with long-term ( $\geq 12$ months) serial intravascular ultrasound. J Am Coll Cardiol 2006;47:1363-1368.

16. Mintz GS, Nissen SE, Anderson WD, et al. American College of Cardiology clinical expert consensus document on standards for acquisition, measurement and reporting of intravascular ultrasound studies (IVUS). J Am Coll Cardiol 2001;37:1478-1492.

17. Baumgart $\mathrm{D}$, Haude $\mathrm{M}, \mathrm{Ge} \mathrm{J}$, et al. Online integration of intravascular ultrasound images into angiographic images. Cathet Cardiovasc Diagn 1996;39:328-329.

18. Devereux RB, Alderman MH. Role of preclinical cardiovascular disease in evolution from risk factor exposure to development of morbid events. Circulation 1993;88:1444-1455.

19. Waters D, Craven TE, Lesperance J. Prognostic significance of progression of coronary atherosclerosis. Circulation 1993;87:1399-1401.

20. Maron DJ, Fazio S, Linton MF. Current perspectives on statins. Circulation 2000;101:207-213.

21. Taylor A, Shaw LJ, Fayad Z, et al. Tracking atherosclerosis regression: a clinical tool in preventive cardiology. Atherosclerosis 2005;180:1-10.

22. Nissen SE, Tuzcu EM, Schoenhagen $P$, et al. Effect of intensive compared with moderate lipidlowering therapy on progression of coronary atherosclerosis A randomized controlled trial. JAMA 2004;291:1071-1080.

23. Jensen LO, Thayssen $P$, Pederson KE, et al. Regression of coronary atherosclerosis by simvastatin: a serial intravascular ultrasound study. Circulation 2004;110:265-270. 
24. Schartl M, Bocksch W, Koschyk DH, et al. Use of intravascular ultrasound to compare effects of different strategies of lipid-lowering therapy on plaque volume and composition in patients with coronary artery disease. Circulation 2001;104:387-392.

25. Larosa JC, Grundy SM, Waters DD, et al. Intensive lipid lowering with atorvastatin in patients with stable coronary disease. N Engl J Med 2005;352:1425-1435.

26. Cannon $\mathrm{CP}$, Braunwald $\mathrm{E}, \mathrm{McCabe} \mathrm{CH}$, et al. Intensive versus moderate lipid lowering with statins after acute coronary syndromes. N Engl J Med 2004;350:1495-1504.

27. Kawasaki M, Sano K, Okubo M, et al. Volumetric quantitative analysis of tissue characteristics of coronary plaques after statin therapy using three-dimensional integrated backscatter intravascular ultrasound. J Am Coll Cardiol 2005;45:1946-1953.

28. von Birgelen C, de Very EA, Mintz GS, et al. ECG-gated three-dimensional intravascular ultrasound: feasibility and reproducibility of the automated analysis of coronary lumen and atherosclerotic plaque dimensions in humans. Circulation 1997;96:2944-2952. 



\section{Chapter 4}

\section{Relation Between Lipoprotein(a) and Fibrinogen and Serial Intravascular Ultrasound Plaque Progression in Left Main Coronary Arteries}

Marc Hartmann, Clemens von Birgelen, Gary S. Mintz, Martin G. Stoel, Holger Eggebrecht, Heinrich Wieneke, Martin Fahy, Till Neumann, Job van der Palen, Hans W. Louwerenburg, Patrick M.J. Verhorst, Raimund Erbel

Reprinted with permission from:

Journal of the American College of Cardiology 2006;48(3):446-452.

(c) 2006 American College of Cardiology Foundation / Elsevier Inc. 



\section{Abstract}

Objectives: Patients with elevated lipoprotein(a) $[L p(a)]$ and fibrinogen levels have an increased risk of coronary heart disease and adverse cardiovascular events. There is evidence that coronary plaque progression is linked to a higher risk for future cardiovascular events.

Background: There are no data demonstrating a relation between $L p(a)$, fibrinogen, and directly measured coronary plaque progression over time.

Methods: We performed a retrospective analysis of serial intravascular ultrasound (IVUS) studies of 60 left main stems (18 \pm 9 months apart) to evaluate plaque progression in relation to $L p(a)$ and fibrinogen levels and association with adverse cardiovascular events.

Results: There was a positive correlation between $\operatorname{Lp}(a)(r=0.58 ; p<0.0001)$, fibrinogen $(r=0.48$; $p<0.0001)$, and changes in plaque-plus-media area. Patients with plaque progression $(n=41)$ had higher $L p(a)(30 \pm 26 \mathrm{mg} / \mathrm{dl}$ vs. $14 \pm 9 \mathrm{mg} / \mathrm{dl} ; \mathrm{p}<0.0012)$ and fibrinogen $(295 \pm 88 \mathrm{mg} / \mathrm{dl}$ vs. $240 \pm 72$ $\mathrm{mg} / \mathrm{dl} ; \mathrm{p}=0.019)$ levels than patients with plaque regression $(n=19)$. Multivariate linear regression analysis showed $\log \operatorname{Lp}(a)$ (regression coefficient=9.45; $p=0.0008$ ) but not fibrinogen to be independently associated with plaque progression. A total of 19 patients suffered from adverse cardiovascular events; they had higher $L p(a)(44 \pm 30 \mathrm{mg} / \mathrm{dl}$ vs. $16 \pm 12 \mathrm{mg} / \mathrm{dl} ; p<0.0001)$ and fibrinogen $(342 \pm 73 \mathrm{mg} / \mathrm{dl}$ vs. $248 \pm 76 \mathrm{mg} / \mathrm{dl} ; \mathrm{p}<0.0001)$ levels. Multivariate logistic regression analysis showed $\log \operatorname{Lp}(a)$ (odds ratio 10.20 , 95\% confidence interval 2.36 to $44.13 ; p=0.0019$ ) and fibrinogen (odds ratio $1.01,95 \%$ confidence interval 1.00 to $1.03 ; p=0.018$ ) were independently associated with adverse cardiovascular events.

Conclusions: Serial IVUS showed a positive correlation between $L p(a)$ and fibrinogen levels and plaque progression. $L p(a)$, but not fibrinogen, remains independently associated with plaque progression. In addition, the present data suggest a considerable incremental value of $L p(a)$ in predicting cardiovascular risk.

\section{Introduction}

Lipoprotein(a) $[L p(a)]$ and fibrinogen have been shown to serve as predictors of coronary heart disease and fatal cardiovascular events. ${ }^{1-10}$ Patients with elevated $L p(a)$ and fibrinogen levels have an increased risk of coronary heart disease ${ }^{11,12}$ and cardiovascular events. ${ }^{12-14}$ There is evidence that plaque progression in coronary arteries is linked to a higher risk of future fatal or nonfatal cardiovascular events. $^{15,16}$ Lipoprotein(a) is associated with plaque progression, as indirectly assessed by lumen changes in short-term ${ }^{17,18}$ and long-term ${ }^{19,20}$ angiographic follow-up, and with calcium score changes as measured by sequential electron beam computerized tomography. ${ }^{21}$ There are no data demonstrating directly the relation between $L p(a)$, fibrinogen, and coronary plaque progression.

Intravascular ultrasound (IVUS) imaging permits measurements of total vessel, lumen, and plaque dimensions in vivo. ${ }^{22}$ Serial IVUS is an ideal tool to directly assess progression or regression of atherosclerotic plaques in native coronary arteries in relation to serum parameters. ${ }^{23-25}$ In the present observational study we retrospectively analyzed serial IVUS ( $\geq 12$ months) data of nonstenotic left main 
coronary arteries to test whether $L p(a)$ and fibrinogen levels predict the progression of coronary atherosclerosis and the onset of adverse cardiovascular events (beyond the information provided by classic risk factors) and whether potential relations are independent of each other.

\section{Methods}

\section{Study population}

We performed a retrospective analysis of $L p(a)$ and fibrinogen levels in relation to serial IVUS studies of atherosclerotic left main coronary plaques in a population that has been reported previously. ${ }^{25}$ All plaques were de novo, hemodynamically nonsignificant, and met the following criteria: 1) serial high-quality IVUS of the entire left main artery $\geq 12$ months apart; 2) calcifications that did not limit quantitative assessment of vessel cross-sectional area (CSA); 3) nonostial plaque location; 4) angiographic lumen diameter stenosis <30\% ("worst view" visual assessment); and 5) no intervention in the very proximal left anterior descending or circumflex coronary arteries, because these interventions could have affected the left main artery. Patients were examined in the Essen University Cardiac Catheterization Laboratory (a tertial referral center) with a follow-up of $18 \pm 9$ months (median 14 months, range 12 to 50 months). As previously reported, this represents consecutive patients who underwent initial IVUS examination during coronary intervention and then returned after a 1- to 2-year period for repeat IVUS examination. ${ }^{25}$ No patient had a myocardial infarction within 3 months before the index examination. At Essen University, patients with previous IVUS who returned for recatheterization were routinely examined with IVUS as part of the clinical protocol. In none of these patients was IVUS performed as part of another study. This IVUS study was approved by the Local Council on Human Research, and all patients signed a written informed consent form as approved by the local medical ethics committee.

\section{Cardiovascular risk factors, parameters, and medication}

In our laboratory we record demographics, cardiovascular risk factors, medications, and results of key laboratory tests of patients examined with IVUS. All baseline blood samples were collected from the same site (periphereal vein) $27 \pm 8 \mathrm{~h}$ before percutaneous catheterization ${ }^{26}$ and analyzed in the central laboratory of Essen University according to international standards. Plasma concentrations of total cholesterol, low-density lipoprotein cholesterol (LDL-C), high-density lipoprotein cholesterol (HDL-C), and triglycerides were measured by standard enzymatic 
methods. Total cholesterol/HDL-C and LDL-C/HDL-C ratios were calculated. Lipoprotein(a) levels were quantitated by nephelometry after addition of an antiserum (Behringwerke, Marburg, Germany). ${ }^{27}$ Fibrinogen was measured by determining the coagulation time of prediluted citrated plasma in the presence of a large amount of thrombin (Behringwerke). Baseline data of all laboratory variables were used for statistical analyses. Cardiovascular risk factors that were recorded included diabetes mellitus and hypertension (both medication-dependent only), history of smoking, and family history of coronary artery disease. Medications were recorded only if drugs were taken for $>50 \%$ of the follow-up interval (e.g., clopidogrel for 4 weeks was not tabulated).

\section{IVUS imaging}

The IVUS imaging was performed as previously described. ${ }^{25}$ In brief, IVUS studies were performed during percutaneous coronary interventions $(\mathrm{PCl})$ of middle or distal left anterior or circumflex arteries after intracoronary injections of $200 \mu \mathrm{g}$ nitroglycerin with commercially available IVUS systems: a mechanical sector scanner (Boston Scientific, San Jose, California) incorporating a 30-MHz singleelement beveled transducer or a solid-state device (Endosonics, Rancho Cordova, California). Importantly, at Essen University, if a patient undergoes imaging with one IVUS system during an index procedure, the same IVUS system is used at follow-up. Slow continuous pullbacks were generally performed using a motorized pullback device (at $0.5 \mathrm{~mm} / \mathrm{s}$ ). In addition, a dedicated image-in-image system (Echo-Map; Siemens, Erlangen, Germany) was used to record the "angiographic" position of the IVUS probe together with the corresponding IVUS image. ${ }^{28}$

\section{Quantitative IVUS analysis}

The left main target site image slice was determined from the initial IVUS study as previously described ${ }^{25}$; this was the site with the smallest lumen CSA within the plaque. Exact matching of the target site on initial and follow-up IVUS studies was ensured using side-by-side comparison of end-diastolic IVUS images of the two IVUS sequences along with the pullback speed, the operators' recorded comments (on videotape), and characteristic calcifications, vascular and perivascular landmarks, and plaque shapes. If required, X-ray sequences of the dedicated image-in-image system (Echo-Map) were revisited to optimize matching. ${ }^{28}$

The lumen CSA was measured by tracing the leading edge of the intima. The external elastic membrane (EEM) CSA was measured by tracing the leading edge of the adventitia. Plaque plus media (P\&M) CSA was calculated (as EEM - lumen CSA). Extrapolation of the EEM boundary behind calcium was possible if each 
individual calcific deposit did not shadow $>75^{\circ}$ of the adventitial circumference. To compensate for variations in follow-up intervals and to obtain comparable data, we calculated absolute and relative changes $(\Delta)$ between initial and follow-up IVUS data; measurements were normalized for the length of the follow-up period (changes per year) and baseline variables.

\section{Statistical analysis}

Analyses were performed with SPSS 10.0.7 (SPSS Inc., Chicago, Illinois). Dichotomous data are presented as frequencies. Quantitative data are presented as mean $\pm 1 \mathrm{SD}$ and were compared using Student $t$ test, Mann-Whitney $U$ test, and regression analysis. Correlation analyses were performed with nonparametric Spearman test ( $r=S$ pearman correlation coefficient). Because the distribution of $L p(a)$ was skewed it was log transformed (Log) to obtain a normal distribution and more reliable estimates for multivariate regression analyses. A $p$ value of $<0.05$ was considered significant.

\section{Results}

Demographics, medication, and laboratory testing of patients

Demographics, medication, and baseline laboratory testing of the study population $(n=60)$ are presented in Table 1 . We found a positive correlation $(r=0.49 ; 95 \%$ confidence interval [Cl] 0.27 to $0.66 ; p=0.0001$ ) between $\mathrm{Lp}(\mathrm{a})$ (median $14.5 \mathrm{mg} / \mathrm{dl}$, range 6.2 to $125.0 \mathrm{mg} / \mathrm{dl}$ ) and fibrinogen levels (median $271.0 \mathrm{mg} / \mathrm{dl}$, range 110.0 to $510.5 \mathrm{mg} / \mathrm{dl})$.

Relation between Lp(a) and serial IVUS data

There was a positive correlation between annual changes in P\&M CSA versus $L p(a)$ levels $(r=0.58 ; 95 \% \mathrm{Cl} 0.39$ to $0.73 ; p<0.0001)$ (Figure 1$)$. There was a negative correlation between annual changes in lumen CSA versus $L p(a)$ levels $(r=-0.24 ; 95 \% \mathrm{Cl}-0.55$ to $-0.10 ; p=0.007)$ (data not shown) and no correlation between annual changes in EEM CSA versus $L p(a)$ levels $(r=0.01 ; 95 \% \mathrm{Cl}-0.25$ to 0.26; $p=0.95$ ) (data not shown). Patients with P\&M CSA increase $(n=41)$ had significantly higher $L p(a)$ levels than patients with P\&M CSA decrease $(n=19)$ $(30 \pm 26 \mathrm{mg} / \mathrm{dl}$ vs. $14 \pm 9 \mathrm{mg} / \mathrm{dl}$; $p<0.0012)$. 
Table 1 (part 1). Patient Demographics, Medications, and Laboratory Testing

\begin{tabular}{|c|c|}
\hline & All Patients $(n=60)$ \\
\hline IVUS follow-up, months & $18 \pm 9$ \\
\hline Age, yrs & $58 \pm 8$ \\
\hline Men, n (\%) & $50(83)$ \\
\hline Body mass index, $\mathrm{kg} / \mathrm{m}^{2}$ & $27 \pm 3$ \\
\hline Previous myocardial infarction, n (\%) & $21(35)$ \\
\hline Smoker, n (\%) & $16(27)$ \\
\hline Systemic arterial hypertension, n (\%) & $43(72)$ \\
\hline Diabetes, n (\%) & $8(13)$ \\
\hline Family history of coronary disease, n (\%) & $14(23)$ \\
\hline \multicolumn{2}{|l|}{ Vessels diseased, n (\%) } \\
\hline 1 & $28(47)$ \\
\hline 2 & $16(27)$ \\
\hline 3 & $16(27)$ \\
\hline \multicolumn{2}{|l|}{ Clinical syndrome, n (\%) } \\
\hline \multicolumn{2}{|l|}{ Stable angina CCS class } \\
\hline I & $17(28)$ \\
\hline II & $29(48)$ \\
\hline III & $9(15)$ \\
\hline IV & $5(8)$ \\
\hline \multicolumn{2}{|l|}{ Medication, n (\%) } \\
\hline Acetylsalicylic acid & $60(100)$ \\
\hline ACE inhibitors & $22(37)$ \\
\hline Angiotensin receptor antagonists & $2(3)$ \\
\hline Beta-blockers & $36(60)$ \\
\hline Calcium-channel blockers & $17(28)$ \\
\hline Diuretics & $18(30)$ \\
\hline Fibrates & $3(5)$ \\
\hline
\end{tabular}


Table 1 (part 2). Patient Demographics, Medications, and Laboratory Testing

\begin{tabular}{lc}
\hline Insulin & All Patients (n = 60) \\
Nitrates & $2(3)$ \\
Oral antidiabetics & $36(60)$ \\
Statins & $3(5)$ \\
Laboratory tests* & $49(82)$ \\
Lipoprotein(a), mg/dl & $25 \pm 23$ \\
Fibrinogen, mg/dl & $278 \pm 87$ \\
Total-C, mg/dl & $199 \pm 39$ \\
LDL-C, mg/dl & $127 \pm 45$ \\
HDL-C, mg/dl & $48 \pm 15$ \\
Total-C/HDL-C (ratio) & $127 \pm 74$ \\
LDL-C/HDL-C (ratio) & $4.5 \pm 1.6$ \\
\hline Triglycerides, mg/dl & $2.9 \pm 1.5$ \\
\hline . & \\
\hline
\end{tabular}

${ }^{*}$ Blood sampling $27 \pm 8 \mathrm{~h}$ before index examination.

ACE=angiotensin-converting enzyme; $C=$ cholesterol; $C C S=$ Canadian Cardiovascular Society; $H D L=$ high-density lipoprotein; IVUS=intravascular ultrasound; $L D L=l o w$-density lipoprotein

\section{Relation between fibrinogen and serial IVUS data}

There was a positive correlation between annual changes in P\&M CSA versus fibrinogen levels $(r=0.48 ; 95 \% \mathrm{Cl} 0.25$ to $0.65 ; p<0.0001)$ (Figure 1$)$. There was no significant correlation between annual changes in lumen CSA versus fibrinogen levels ( $r=-0.23 ; 95 \% \mathrm{Cl}-0.46$ to $0.02 ; p=0.07$ ) (data not shown) and no correlation between annual changes in EEM CSA versus fibrinogen levels $(r=0.11 ; 95 \% \mathrm{Cl}$ -0.15 to $0.35 ; p=0.42$ ) (data not shown). Patients with P\&M CSA increase $(n=41)$ had significantly higher fibrinogen levels than patients with P\&M CSA decrease $(\mathrm{n}=19)(295 \pm 88 \mathrm{mg} / \mathrm{dl}$ vs. $240 \pm 72 \mathrm{mg} / \mathrm{dl} ; \mathrm{p}=0.019)$. 

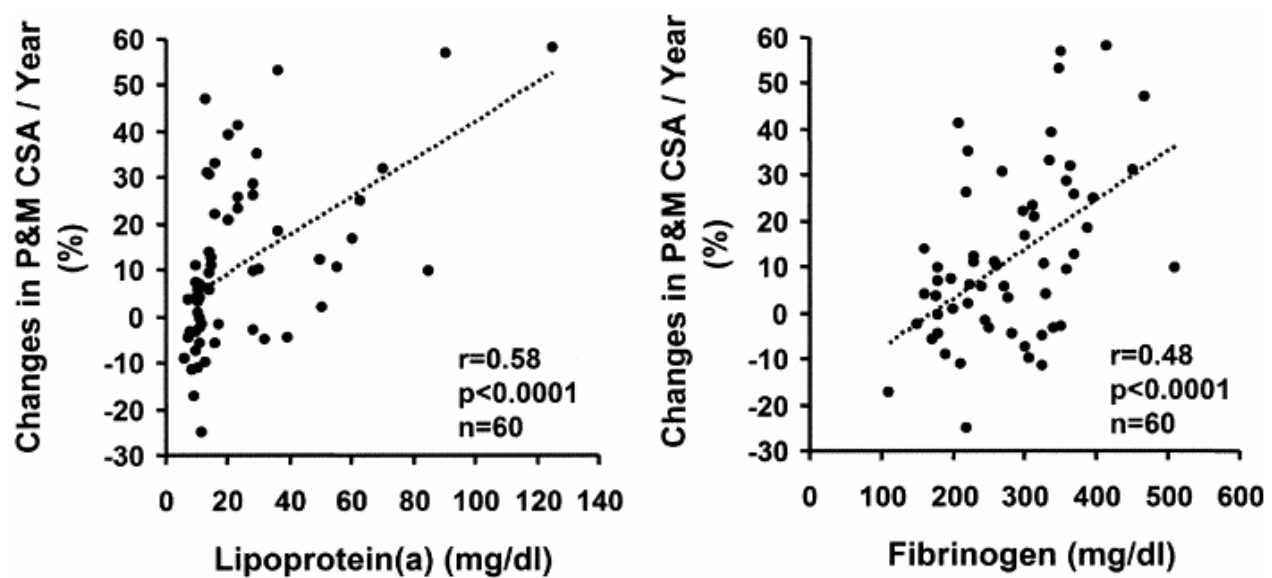

Figure 1. Lipoprotein(a) and fibrinogen serum levels versus annual changes in plaque plus media (P\&M) cross-sectional area (CSA).

$r=$ Spearman correlation coefficient. Dotted regression lines were derived from linear regression analysis.

\section{Multivariate analysis of predictors of P\&M CSA increase}

Multivariate linear regression analysis was used to determine predictors of the increase in P\&M CSA (Table 2). The following were tested in the multivariate model: Log Lp(a), fibrinogen, LDL-C, HDL-C, total cholesterol, total cholesterol/HDL-C ratio, LDL-C/HDL-C ratio, smoking, hypertension, diabetes, family history, age, and use of statins (LDL-C, HDL-C, and smoking were shown in a previous publication to be univariate predictors of the increase in P\&M CSA in these patients $\left.{ }^{16}\right)$. Independent predictors were $\log \operatorname{Lp}(a)(p=0.0008)$ and smoking $(p=0.0001)$; LDL-C $(p=0.078)$ showed a trend, whereas fibrinogen was not an independent predictor (Table 2).

Table 2. Multivariate Predictors of Plaque Plus Media Cross-Sectional Area Increase.

\begin{tabular}{lccc}
\hline \multicolumn{1}{c}{$\begin{array}{c}\text { Multivariate } \\
\text { Predictors* }\end{array}$} & $\begin{array}{c}\text { Regression } \\
\text { Coefficient }\end{array}$ & $\begin{array}{c}\text { Standard } \\
\text { Error }\end{array}$ & $\mathbf{p}$ \\
Intercept & -30.4322 & 7.8713 & 0.0003 \\
Log lipoprotein(a) & 9.4452 & 2.6743 & 0.0008 \\
Smoker & 17.1133 & 4.1103 & 0.0001 \\
LDL-cholesterol & 0.0758 & 0.0422 & 0.0778 \\
\hline
\end{tabular}

$\overline{L D L}=$ low-density lipoprotein. * Multivariate predictors are chosen by stepwise linear regression with entry/stay criteria of 0.2/0.1. 


\section{$L p(a)$ and fibrinogen and adverse cardiovascular events}

During follow-up, 6 patients (10\%) suffered from acute myocardial infarction, 7 $(12 \%)$ from unstable angina, and $6(10 \%)$ from $\mathrm{PCl}$ of a new de novo coronary lesion. The mean interval between baseline laboratory test and adverse event during follow-up was $450 \pm 98$ days. Patients with adverse cardiovascular events $(n=19)$ had higher $L p(a)(44 \pm 30 \mathrm{mg} / \mathrm{dl}$ vs. $16 \pm 12 \mathrm{mg} / \mathrm{dl} ; \mathrm{p}<0.0001)$ and fibrinogen levels $(342 \pm 73 \mathrm{mg} / \mathrm{dl}$ vs. $248 \pm 76 \mathrm{mg} / \mathrm{dl} ; \mathrm{p}<0.0001)$ than patients without adverse events $(n=41)$ (Figure 2, Table 3 ). In addition, patients with adverse events had significantly higher total cholesterol/HDL-C and LDL-C/HDL-C ratios and significantly more annual increase in P\&M CSA than patients without events (Table $3)$.
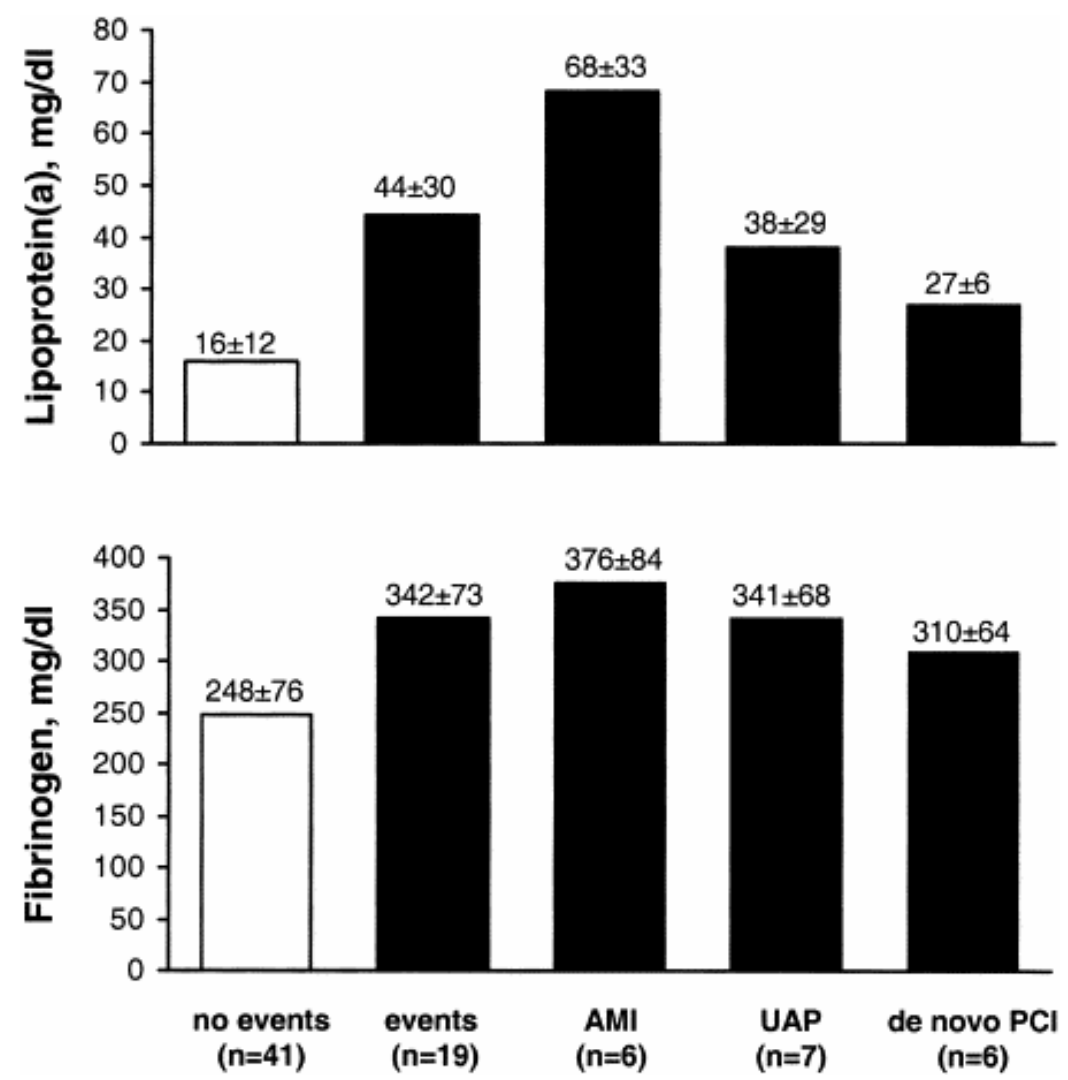

Figure 2. Lipoprotein(a) and fibrinogen serum levels in patients with and without adverse cardiovascular events.

$A M I=$ acute myocardial infarction; $P C I=$ percutaneous coronary intervention; $U A P=$ unstable angina pectoris. 
Table 3. Laboratory Tests and Serial IVUS Data of Patients With and Without Clinical End Points and/or Revascularization Procedures During Follow-Up.

\begin{tabular}{|c|c|c|c|}
\hline & $\begin{array}{l}\text { Patients With Clinical } \\
\text { End Points and/or } \\
\text { Revascularization } \\
\text { Procedures }(n=19)\end{array}$ & $\begin{array}{l}\text { Patients Without Clinical } \\
\text { End Points and/or } \\
\text { Revascularization } \\
\text { Procedures }(n=41)\end{array}$ & $\mathbf{p}$ \\
\hline \multicolumn{4}{|l|}{ Laboratory tests* } \\
\hline Lipoprotein(a), mg/dl & $44 \pm 30$ & $16 \pm 12$ & $<0.0001 \dagger$ \\
\hline Fibrinogen, mg/dl & $342 \pm 73$ & $248 \pm 76$ & $<0.0001 \ddagger$ \\
\hline Total-C, mg/dl & $208 \pm 39$ & $196 \pm 38$ & $0.27 \ddagger$ \\
\hline LDL-C, mg/dl & $140 \pm 42$ & $120 \pm 45$ & $0.10 \ddagger$ \\
\hline $\mathrm{HDL}-\mathrm{C}, \mathrm{mg} / \mathrm{dl}$ & $44 \pm 18$ & $50 \pm 13$ & $0.20 \ddagger$ \\
\hline Total-C/HDL-C (ratio) & $5.2 \pm 1.7$ & $4.2 \pm 1.4$ & $0.02 \ddagger$ \\
\hline LDL-C/HDL-C (ratio) & $3.8 \pm 1.6$ & $2.6 \pm 1.4$ & $0.02 \ddagger$ \\
\hline Triglycerides, mg/dl & $104 \pm 55$ & $137 \pm 80$ & $0.11 \ddagger$ \\
\hline \multicolumn{4}{|l|}{ Serial IVUS } \\
\hline$\triangle \mathrm{P} \& \mathrm{M} \mathrm{CSA} / \mathrm{yr}, \mathrm{mm}^{2}$ & $1.7 \pm 1.0$ & $0.3 \pm 1.5$ & $0.001 \ddagger$ \\
\hline$\triangle \mathrm{P} \& \mathrm{M}$ CSA/yr, $\%$ & $24.0 \pm 16.6$ & $5.6 \pm 16.5$ & $0.0002 \ddagger$ \\
\hline$\Delta$ Lumen CSA/yr, mm² & $-0.8 \pm 4.0$ & $-0.1 \pm 2.4$ & $0.41 \ddagger$ \\
\hline$\Delta$ Lumen CSA/yr, \% & $-3.1 \pm 20.5$ & $0.9 \pm 16.9$ & $0.43 \ddagger$ \\
\hline$\Delta \mathrm{EEM} \mathrm{CSA} / \mathrm{yr}, \mathrm{mm}^{2}$ & $0.9 \pm 4.3$ & $0.2 \pm 2.8$ & $0.48 \ddagger$ \\
\hline$\triangle \mathrm{EEM} \mathrm{CSA} / \mathrm{yr}, \%$ & $5.5 \pm 15.7$ & $1.6 \pm 11.0$ & $0.27 \ddagger$ \\
\hline
\end{tabular}

$\Delta=$ change; $C S A=$ cross-sectional area; $E E M=$ external elastic membrane; $P \& M=$ plaque plus media; other abbrevations as in Table 1. * Blood sampling $27 \pm 8 \mathrm{~h}$ before index examination; $\dagger$ compared using Mann-Whitney $U$ test; $\ddagger$ compared using Student $t$ test. 
Multivariate analysis of predictors of adverse cardiovascular events

Multivariate logistic regression analysis was used to determine predictors of adverse cardiovascular events during follow-up (Table 4). The following were tested in the multivariate model: Log $L p(a)$, fibrinogen, LDL-C, HDL-C, total cholesterol, total cholesterol/HDL-C ratio, LDL-C/HDL-C ratio, smoking, hypertension, diabetes, familiy history, age, use of statins, and increase in P\&M CSA. Independent predictors were $\log \operatorname{Lp}(a)(p=0.0019)$ and fibrinogen $(p=0.018)$ (Table 4).

Table 4. Multivariate Predictors of Adverse Cardiovascular Events (Clinical End Points and/or Revascularization Procedures) During Follow-Up.

\begin{tabular}{lcccc}
\hline \multicolumn{1}{c}{$\begin{array}{c}\text { Multivariate } \\
\text { Predictors* }\end{array}$} & $\begin{array}{c}\text { Odds Ratio } \\
(\mathbf{9 5 \%} \text { Cl) }\end{array}$ & $\begin{array}{c}\text { Regression } \\
\text { Coefficient }\end{array}$ & $\begin{array}{c}\text { Standard } \\
\text { Error }\end{array}$ & $\mathbf{p}$ \\
Intercept & $10.20(2.36-44.13)$ & -7.2948 & 3.9502 & 0.0648 \\
Log lipoprotein(a) & 2.3228 & 0.7471 & 0.0019 \\
Fibrinogen & $1.01(1.00-1.03)$ & 0.0141 & 0.0060 & 0.0183 \\
\hline
\end{tabular}

$\mathrm{Cl}=$ confidence interval. ${ }^{*}$ Multivariate predictors are chosen by stepwise logistic regression with entry/stay criteria of 0.2/0.1.

\section{Discussion}

\section{$L p(a)$ and coronary plaque progression}

Previous studies revealed $L p(a)$ as a predictor of coronary heart disease $e^{3,7}$ angiographic severity, and extent of the disease process ${ }^{3}$. However, other studies failed to demonstrate an association between $L p(a)$ and cardiovascular disease. $^{29,30}$ In good agreement with our findings, Lp(a) levels showed a strong correlation with serial plaque progression assessed in previous angiographic ${ }^{17-20}$ and electron beam tomographic studies. ${ }^{21}$ Uchida et al. ${ }^{18}$ showed Lp(a) to be an independent predictor of angiographic lesion progression at 6 months follow-up, and Matsumoto et al. ${ }^{19}$ showed that the $L p(a)$ level was significantly associated with angiographic disease progression after more than 24 months. In support of this, we found a positive correlation between $L p(a)$ levels versus annual changes in P\&M CSA. In addition to smoking, $L p(a)$ was an independent predictor of plaque progression in a multivariate analysis (LDL-C showed only a trend). The cluster of $L p(a)$ levels around $10 \mathrm{mg} / \mathrm{dl}$ does not permit defining an exact threshold below which no plaque progression occurs. However, the mean $L p(a)$ level of patients with plaque progression was $30 \mathrm{mg} / \mathrm{dl}$, whereas it was $14 \mathrm{mg} / \mathrm{dl}$ in patients without 
plaque progression $(p=0.0012)$. Such $L p(a)$ values (around $30 \mathrm{mg} / \mathrm{dl}$ ) were also found in previous serial angiographic studies to be associated with long-term plaque progression. ${ }^{19}$

The pathophysiologic role of $L p(a)$ in atherosclerotic disease progression is explained by the accumulation of $L p(a)$ in the vessel wall and its ability to promote cholesterol accumulation in macrophages forming foam cells and subsequent fatty streaks ${ }^{31,32}$ and to promote smooth muscle cell proliferation and migration in atherosclerotic lesions by inactivating transforming growth factor-beta. ${ }^{33}$ Lipoprotein(a) is considered to have an inhibitory effect on fibrinolytic mechanisms owing to its structural similarity to plasminogen causing competition with plasminogen activators; intraluminal thrombus formation may also cause reparative proliferative response of the vascular wall. ${ }^{17,31,32}$

\section{Fibrinogen and coronary plaque progression}

It has been demonstrated that fibrinogen may also be associated with severity and extent of coronary atherosclerosis ${ }^{8,9}$ and with electron beam tomography-assessed calcium score. ${ }^{10}$ In support of this, we found a positive correlation between fibrinogen levels versus annual changes in P\&M CSA. However, fibrinogen was not an independent predictor of plaque progression in a multivariate analysis. Fibrinogen affects blood rheology and platelet aggregation, which play a role in atherogenesis. ${ }^{34}$ Nevertheless, the mechanisms by which fibrinogen may promote atherosclerosis progression remains to be determined. ${ }^{6}$

\section{Vascular remodeling}

In the present study we found no relation between $L p(a)$ or fibrinogen levels versus actual arterial remodeling ( $\triangle E E M C S A)$. One nonserial IVUS study found no relation between $L p(a)$ levels and the remodeling index, defined as the ratio of the lesion EEM CSA to the reference EEM CSA. ${ }^{35}$ In addition, other blood chemistries that are associated with atherogenesis (total cholesterol, LDL-C, HDL-C, triglycerides, and C-reactive protein) also had no impact on arterial remodeling. ${ }^{35}$

Relation between Lp(a), fibrinogen, and adverse cardiovascular events

A correlation between $L p(a)$ and fibrinogen concentrations has been reported ${ }^{36}$ but was not consistently found in the majority of previous studies. ${ }^{37}$ In the present study population, $L p(a)$ levels correlated directly with fibrinogen levels. In vitro studies showed that over time $\operatorname{Lp}(a)$ binds with fibrinogen in a concentrationdependent manner. ${ }^{38}$ The deposition of $L p(a)$ within the fibrin clot is believed to be 
a contributing factor in atherogenesis, promoting plaque progression. ${ }^{31,32,38}$ Study patients with plaque progression had significantly higher $L p(a)$ and fibrinogen levels. However, only $L p(a)$ was an independent predictor of plaque progression.

On the other hand, the association between $\operatorname{Lp}(a)$ and fibrinogen levels may indicate a multiple unstable plaque phenotype (advanced disease); fibrinogen may be elevated as part of the acute phase response, and $L p(a)$ has also been shown to rise after plaque destabilization. ${ }^{26}$ Two clinical studies showed that patients with elevated serum $L p(a)$ levels, when associated with high fibrinogen levels, had a significantly increased cardiovascular disease risk. ${ }^{11,12}$ Peak serum levels of Lp(a) and fibrinogen seem to coincide with the morning peak frequencies of myocardial infarction and stroke. ${ }^{14}$ Accordingly, we found that patients with adverse cardiovascular events had significantly higher serum levels of both $L p(a)$ and fibrinogen than patients who were event free during follow-up. In a multivariate model, $L p(a)$ and fibrinogen were independent predictors of adverse cardiovascular events. Our data suggest in particular an incremental value of $L p(a)$ in predicting cardiovascular risk (beyond the information provided by classic risk factors).

\section{Study limitations}

By most standards, this was a large serial IVUS study; however, all current studies with long-term serial IVUS assessment of atherosclerosis are limited to a relatively small number of patients. This was a retrospective analysis, and we included only patients with established coronary artery disease and repeat cardiac catheterization $\geq 12$ months after baseline. About a third of the subjects experienced a clinical event during a follow-up of only 18 months in spite of adequate therapy and prevention. Accordingly, this is a very high-risk group. Because of this selection bias, the findings of the current study may not be applicable to a standard population with coronary artery disease or even the general population without established disease. Other superior markers of inflammation (e.g., high-sensitivity C-reactive protein) were not available for the present study population. We used 2 IVUS systems in the present study; although this approach may have minor shortcomings, every effort was taken to obtain the most reliable data possible, as previously discussed in detail. ${ }^{25}$ Three-dimensional analyses may be superior for the assessment of coronary dimensions and provide volumetric data. ${ }^{39}$ We studied only left main artery disease as representative of nonintervened coronary segments. 


\section{Conclusions}

We found a positive correlation between $L p(a)$ and fibrinogen levels versus annual changes in P\&M CSA. Lipoprotein(a) was an independent predictor of plaque progression (regression coefficient $=9.45$ ), with a mean $L p(a)$ level of $30 \mathrm{mg} / \mathrm{dl}$ in patients with plaque progression. Lipoprotein(a) and fibrinogen were independent predictors of adverse cardiovascular events during follow-up in this group of patients. The data suggest in particular a considerable incremental value of $L p(a)$ in predicting cardiovascular risk.

\section{References}

1. Ariyo AA, Thach C, Tracy $R$ and Cardiovascular Health Study Investigators, Lp(a) lipoprotein, vascular disease, and mortality in the elderly. N Engl J Med 2003;349:2108-2115.

2. Rhoads GG, Dahlen G, Berg K, et al. Lp(a) lipoprotein as a risk factor for myocardial infarction. JAMA 1986;256:2540-2544.

3. Rader DJ, Hoeg JM, Brewer Jr. HB. Quantitation of plasma apolipoproteins in the primary and secondary prevention of coronary artery disease. Ann Intern Med 1994;120:1012-1025.

4. Wilhelmsen L, Svardsudd K, Korsan-Bengsten K, et al. Fibrinogen as risk factor for stroke and myocardial infarction. N Engl J Med 1984;311:501-505.

5. Kannel WB, Wolf PA, Castelli WP, et al. Fibrinogen and risk of cardiovascular disease. The Framingham Study. JAMA;1987;258:1183-1186.

6. Ernst E, Resch KL. Fibrinogen as a cardiovascular risk factor a meta-analysis and review of the literature. Ann Intern Med 1993;188:956-963.

7. Schwartzman RA, Cox ID, Poloniecki J, et al. Elevated plasma lipoprotein(a) is associated with coronary artery disease in patients with chronic stable angina pectoris. $\mathrm{J}$ Am Coll Cardiol 1998;31:1260-1266.

8. Handa K, Kono S, et al. Plasma fibrinogen levels as independent indicator of severity of coronary atherosclerosis. Atherosclerosis 1989;77:209-213.

9. Bolibar I, Kienast J, Thompson SG, et al. and ECAT Angina Pectoris Study Group. Relation of fibrinogen to presence and severity of coronary artery disease is independent of other coexisting heart disease. Am Heart J 1993;125:1601-1605.

10. Schmermund A, Baumgart D, Görge G, et al. Measuring the effect of risk factors on coronary atherosclerosis coronary calcium score versus angiographic disease severity. J Am Coll Cardiol 1998;31:1267-1273.

11. Cantin B, Despres JB, Lamarche B, et al. Association of fibrinogen and lipoprotein(a) as a coronary heart disease risk factor in men (the Quebec Cardiovascular Study). Am J Cardiol 2002;89:662666.

12. Shai I, Rimm EB, Hankinson SE, et al. Lipoprotein(a) and coronary heart disease among women beyond a cholesterol carrier? Eur Heart J 2005;26:1633-1639.

13. Cremer $P$, Nagel D, Labrot $B$, et al. Lipoprotein $L p(a)$ as predictor of myocardial infarction in comparison to fibrinogen, LDL cholesterol and other risk factors results from the prospective Göttingen Risk Incidence and Prevalence Study (GRIPS). Eur J Clin Invest 1994;24:444-453.

14. Bremner WF, Sothern RB, Kanabrocki EL, et al. Relation between circadian patterns in levels of circulating lipoprotein(a), fibrinogen, platelets, and related lipid variables in men. Am Heart $\mathrm{J}$ 2000;139:164-173. 
15. Ballantyne $\mathrm{CM}$. Clinical trials endpoints angiograms, events and plaque instability. Am J Cardiol 1998;82:5-11M.

16. von Birgelen C, Hartmann M, Mintz GS, et al. Relationship between cardiovascular risk as predicted by established risk scores versus plaque progression as measured by serial intravascular ultrasound in left main coronary arteries. Circulation 2004;110:1579-1585.

17. Terres $\mathrm{W}$, Tatsis $\mathrm{E}$, Pfalzer $\mathrm{B}$, et al. Rapid angiographic progression of coronary artery disease in patients with elevated lipoprotein(a). Circulation 1995;91:948-950.

18. Uchida $\mathrm{T}$, Inoue $\mathrm{T}$, Kamishirado $\mathrm{H}$, et al. Prediction of short-term progression or regression of atherosclerotic coronary artery disease by lipoprotein(a) a quantitative coronary angiographic study. Angiology 2003;54:641-646.

19. Matsumoto $\mathrm{Y}$, Daida $\mathrm{H}$, Watanabe $\mathrm{Y}$, et al. High level of lipoprotein(a) is a strong predictor for progression of coronary artery disease. J Atheroscler Thromb 1998;5:47-53.

20. Tamura A, Watanabe T, Mikuriya $Y$, et al. Serum lipoprotein(a) concentrations are related to coronary disease progression without new myocardial infarction. Br Heart J 1995;74:365-369.

21. Chironi G, Simon A, Denarie N, et al. Determinants of progression of coronary artery calcifications in asymptomatic men at high cardiovascular risk. Angiology 2002;53:667-683.

22. Mintz GS, Nissen SE, Anderson WD, et al. American College of Cardiology clinical expert consensus document on standards for acquisition, measurement and reporting of intravascular ultrasound studies (IVUS). J Am Coll Cardiol 2001;37:1478-1492.

23. Schartl M, Bocksch W, Koschyk DH, et al. Use of intravascular ultrasound to compare effects of different strategies of lipid-lowering therapy on plaque volume and composition in patients with coronary artery disease. Circulation 2001;104:387-392.

24. Okkels Jensen L, Thayssen P, Pederson KE, et al. Regression of coronary atherosclerosis by simvastatin a serial intravascular ultrasound study. Circulation 2004;110:265-270.

25. von Birgelen C, Hartmann M, Mintz GS, et al. Relation between progression and regression of atherosclerotic left main coronary artery disease and serum cholesterol levels as assessed with serial long-term ( $\geq 12$ months) follow-up intravascular ultrasound, Circulation 2003;108:2757-2762.

26. Tsimikas S, Lau HK, Han KR, et al. Percutaneous coronary intervention results in acute increases in oxidized phospholipids and lipoprotein(a) short-term and long-term immunologic responses to oxidized low-density lipoprotein. Circulation 2004;109:3164-3170.

27. Fink PC, Römer $M$, Haeckel $R$, et al. Measurement of proteins with Behring nephelometer $A$ multicentre evaluation. J Clin Chem Clin Biochem 1989;27:261-276.

28. Baumgart $\mathrm{D}$, Haude $\mathrm{M}, \mathrm{Ge} \mathrm{J}$, et al. Online integration of intravascular ultrasound images into angiographic images, Cath Cardiovasc Diagn 1996;39:328-329.

29. Jauhiainen M, Koskinen $P$, Ehnholm $C$, et al. Lipoprotein(a) and coronary heart disease risk a nested case-control study of the Helsinki Heart Study participants. Atherosclerosis 1991;89:59-67.

30. Ridker PM, Hennekens $\mathrm{CH}$, Stampfer MJ. A prospective study of lipoprotein(a) and the risk of myocardial infarction. JAMA 1993;270:2195-2199.

31. Liu AC, Lawn RM. Lipoprotein(a) and atherogenesis. Trends Cardiovasc Med 1994;4:40-44.

32. Maher VMG, Brown BG. Lipoprotein(a) and coronary heart disease. Curr Opin Lipidol 1995;6:229235.

33. Grainger DJ, Kirschenlohr HL, Metcalfe JC, et al. Proliferation of human smooth muscle cells promoted by lipoprotein(a). Science 1993;260:1655-1658.

34. Koenig W, Ernst E. The possible role of hemorheology in atherothrombogenesis. Atherosclerosis 1992;94:93-107.

35. Hong MK, Park SW, Lee CW, et al. Elevated homocysteine levels might be associated with coronary artery remodeling in patients with stable angina an intravascular ultrasound study. Clin Cardiol 2002;25:225-229.

36. Braeckman L, De Bacquer D, Rosseneu M, et al. Determinants of lipoprotein(a) levels in a middleaged working population. Eur Heart J 1996;17:1808-1813. 
37. Donders SH, Lustermans FA, van Wersch JW. Low order correlations of lipoprotein(a) with other blood lipids and with coagulation and fibrinolysis parameters in hypertensive and diabetic patients. Blood Coagul Fibrinolysis 1992;3:249-256.

38. Romanic AM, Arleth AJ, Willette RN, et al. Factor XIIla cross-links lipoprotein(a) with fibrinogen and is present in human atherosclerotic lesions. Circ Res 1998;83:264-269.

39. von Birgelen C, de Very EA, Mintz GS, et al. ECG-gated three-dimensional intravascular ultrasound feasibility and reproducibility of the automated analysis of coronary lumen and atherosclerotic plaque dimensions in humans. Circulation 1997;96:2944-2952. 



\title{
Chapter 5
}

Relationship Between Cardiovascular Risk as Predicted by Established Risk Scores Versus Plaque Progression as Measured by Serial Intravascular Ultrasound in Left Main Coronary Arteries

\author{
Clemens von Birgelen, Marc Hartmann, Gary S. Mintz, \\ Gert K. van Houwelingen, Nadine Deppermann, Axel Schmermund, \\ Dirk Böse, Holger Eggebrecht, Till Neumann, Mario Gössl, \\ Heinrich Wieneke, Raimund Erbel
}

Reprinted with permission from:

Circulation 2004;110(12):1579-1585.

(c) 2004 American Heart Association / Lippincott Williams \& Wilkins Inc. 



\section{Abstract}

Background: Intravascular ultrasound (IVUS) is increasingly used as an end point in studies aimed at reducing progression or inducing regression of coronary artery disease. However, data linking serial changes by IVUS with clinical outcomes are scarce.

Methods and Results: In the absence of a validated risk score for secondary prevention, we compared 3 established risk scores for primary prevention (PROCAM, SCORE, and Framingham) with plaque progression and lumen reduction as assessed with serial IVUS (follow-up, 18 \pm 9 months) in atherosclerotic left main coronary arteries of 56 patients with established atherosclerosis. For all 3 algorithms, patients at highest estimated risk of events showed greater plaque progression than patients at lowest risk $(P<0.05$ to $<0.01)$. There were positive linear relationships between the risk of clinical events and plaque progression $(r=0.41$ to $0.60 ; P<0.002$ to $<0.0001)$. This translated into a greater decrease in lumen dimensions with increasing risk $(P<0.05$, PROCAM and SCORE). Risk prediction using the PROCAM algorithm showed the strongest relation with serial IVUS. During follow-up, 18 patients suffered from adverse cardiovascular events; these patients had an annual plaque progression that was significantly greater than other patients $(25.2 \pm 19.4 \%$ versus $5.9 \pm 15.6 \%, P<0.001)$.

Conclusions: There was a positive linear relationship between the estimated risk of clinical events derived from all 3 established risk-score algorithms and the extent of plaque progression measured by serial IVUS. This translated into stenosis progression (reduction in lumen dimensions) with increasing clinical risk.

\section{Introduction}

Coronary artery disease is a major cause of death and disability in Western-lifestyle countries. Intravascular ultrasound (IVUS) permits measurements of both lumen and plaque dimensions in vivo. ${ }^{1}$ Serial IVUS studies can be used to assess the progression of atherosclerotic plaque dimensions in native coronary arteries. ${ }^{2-6}$ Several pharmacological intervention studies with clinical end points have demonstrated improved patient outcomes with risk factor modification. ${ }^{7-10}$ In addition, progression-regression studies with angiographic end points have demonstrated the relationship between angiographic disease progression and clinical outcome. ${ }^{11}$ However, thus far, there is a lack of data linking changes in plaque dimensions as measured with IVUS with clinical outcome. ${ }^{5}$ Guidelines on prevention of coronary heart disease use predicted 10-year risk of coronary events to identify candidates for risk factor modification. ${ }^{12-15}$ Ideally, a predictor of the overall risk of clinical events should be based on a multifactorial model. ${ }^{16,17}$ Published "primary prevention" risk-scoring methods use equations derived from large cohort studies, such as the recent European Systematic COronary Risk Evaluation (SCORE) Project, ${ }^{16}$ the German PROspective CArdiovascular Münster (PROCAM) Study, ${ }^{18}$ and the US Framingham Heart and Offspring Studies. ${ }^{19}$ In the absence of a validated risk score for secondary prevention, we used these established primary-event risk scores ${ }^{17-19}$ to assess the relationship between the 
estimated risk of clinical events and plaque progression as assessed with serial IVUS in atherosclerotic left main stems of patients with symptomatic coronary artery disease.

\section{Methods}

\section{Study population}

We compared established risk scores with serial IVUS studies of left coronary atherosclerotic plaques in a population that has previously been reported. ${ }^{6}$ All plaques were de novo, were hemodynamically nonsignificant, and met the following criteria: (1) serial high-quality IVUS of the entire left main artery $\geq 12$ months apart; (2) calcifications that did not limit quantitative assessment of vessel cross-sectional area (CSA); (3) nonostial plaque location; (4) angiographic lumen diameter stenosis $<30 \%$ ("worst-view" visual assessment); and (5) no intervention in the very proximal left anterior descending or circumflex coronary arteries because these interventions could have affected the left main artery. Of these cases $(n=60)$, the present analysis includes the data of 56 patients $<65$ years old, because most risk scores are validated only for patients up to that age. Patients were examined in the Essen University Cardiac Catheterization Laboratory, with a follow-up of $18 \pm 9$ months (median, 14 months; range, 12 to 50 months). The Local Council on Human Research approved this IVUS study, and all patients signed a written informed consent form as approved by the Local Medical Ethics Committee. As previously reported, this represents a consecutive series of patients who underwent initial IVUS examination during coronary intervention and then returned after $\geq 1$ year for repeat intervention, during which another IVUS study was performed. ${ }^{6}$

\section{Cardiovascular Risk Factors, Parameters, and Medication}

In our laboratory, we prospectively record demographics, cardiovascular risk factors, medications, and results of key laboratory tests of patients examined with IVUS. Baseline laboratory tests were performed in all patients treated by percutaneous interventions and were analyzed in the central laboratory of Essen University according to international standards. Medications were recorded only if drugs were taken for $>50 \%$ of the follow-up interval (e.g., clopidogrel for 4 weeks was not tabulated). Tabulated cardiovascular risk factors and parameters included sex, age, systolic blood pressure, serum total cholesterol level, serum LDL cholesterol level, serum HDL cholesterol level, serum triglyceride level, history of smoking during the previous 12 months (and also the previous month), family history of coronary artery disease (myocardial infarction of first-degree relative $<60$ 
years of age), and diabetes mellitus (known diabetes or repeated fasting blood glucose levels $>120 \mathrm{mg} / \mathrm{dL}$ ). Plasma concentrations of total cholesterol, LDL cholesterol, HDL cholesterol, and triglycerides were measured by standard enzymatic methods

\section{Cardiovascular Risk Assessment by PROCAM Score}

The PROCAM score was calculated as previously described. ${ }^{18}$ It was derived from the European PROCAM study, performed in a population of $\approx 5000$ participants in Münster, Germany. The score considers sex, age, LDL cholesterol, HDL cholesterol, triglycerides, systolic blood pressure, smoking, diabetes mellitus, and family history. The categories for continuous variables of age, systolic blood pressure, LDL cholesterol, and HDL cholesterol were based on the National Cholesterol Education Program III guidelines; ${ }^{15}$ the categories for triglycerides were based on the guidelines of the International Task Force for Prevention of Coronary Heart Disease. ${ }^{20}$ For each patient, 10 -year risk of coronary events (fatal/nonfatal myocardial infarction or sudden death) was predicted. ${ }^{18}$

\section{Cardiovascular Risk by SCORE Risk Assessment System}

The SCORE risk was determined from a risk assessment algorithm previously described. ${ }^{16}$ The SCORE risk assessment system considers sex, age, total cholesterol, systolic blood pressure, and smoking. We used the table for populations at high cardiovascular disease risk on the basis of total/HDL cholesterol levels. It is derived from a large (>205 000 participants) data set of prospective European studies. It predicted the 10-year risk of fatal cardiovascular events. ${ }^{16}$

\section{Cardiovascular Risk Assessment by Framingham Score}

The Framingham score was calculated by use of an algorithm previously described. ${ }^{15}$ The score considers sex, age, total cholesterol, HDL cholesterol, systolic blood pressure, and smoking. The Framingham score is based on data from a sample of the Framingham Heart and Offspring studies. ${ }^{19}$ It was used to predict the 10-year risk of coronary events (fatal/nonfatal myocardial infarction or sudden death). 


\section{IVUS Imaging}

IVUS imaging was performed as previously described. ${ }^{6}$ In brief, IVUS studies were performed during percutaneous coronary interventions of mid or distal left anterior descending or circumflex arteries after intracoronary injections of $200 \mu \mathrm{g}$ nitroglycerin with commercially available IVUS systems: a mechanical sector scanner (Boston Scientific Corp) incorporating a $30-\mathrm{MHz}$ single-element beveled transducer or a solid-state device (Endosonics). Importantly, at Essen University, if a patient undergoes imaging with one IVUS system during an index procedure, the same IVUS system is used at follow-up. Slow, continuous pullbacks were generally performed with a motorized pullback device (at $0.5 \mathrm{~mm} / \mathrm{s}$ ). In addition, a dedicated image-in-image system (Echo-Map, Siemens) ${ }^{21}$ was used to record the "angiographic" position of the IVUS probe together with the corresponding IVUS image, especially at sites of characteristic landmarks (ie, calcifications or unusual plaque shapes) and/or the target site. Follow-up IVUS studies were performed (using the same IVUS system as at the initial measurement) during repeat coronary interventions and during IVUS examinations of ambiguous coronary lesions or (clinically driven) follow-up catheterizations.

\section{Quantitative IVUS Analysis}

The left main artery target site image slice was determined from the initial IVUS study; this was the site with the smallest lumen CSA within the plaque. ${ }^{6}$ If there were several slices with equal lumen size, the one with the largest external elastic membrane (EEM) and plaque plus media (P\&M=EEM minus lumen) CSA was analyzed. $^{1,22,23}$ Exact matching of the target site on initial and follow-up IVUS studies was ensured by use of side-by-side comparison of the 2 IVUS sequences along with the pullback speed; the operators' recorded comments (on video tape); and characteristic calcifications, vascular and perivascular landmarks, and plaque shapes. If required, $x$-ray sequences of the dedicated image-in-image system (Echo-Map) were revisited to optimize matching. ${ }^{21}$

The lumen CSA was measured by tracing the leading edge of the intima. The EEM CSA was measured by tracing the leading edge of the adventitia. Extrapolation of the EEM boundary behind calcium was possible if each individual calcific deposit did not shadow $>75^{\circ}$ of the adventitial circumference. In our laboratory, the intraclass correlation coefficient is 0.99 for repeated measurements of EEM, 0.96 for lumen, and 0.99 for P\&M CSA. To compensate for variations in follow-up intervals and to obtain comparable data, we calculated absolute and relative changes $(\Delta)$ between initial and follow-up IVUS data; measurements were normalized for the length of the follow-up period (changes per year) and baseline 
variables.

To evaluate the potential effect of individual risk factors on annual plaque progression, we compared changes in P\&M CSA of patients with high versus low values of individual risk factors. For noncontinuous variables, we compared patients with presence versus absence of individual risk factors; for continuous variables, we applied the (rounded) median of that parameter to create groups (value $<$ median versus value $\geq$ median). The low number of female patients $(n=6)$ did not justify further testing for sex differences.

\section{Statistical Analysis}

Analyses were performed with SPSS 10.0.7 (Microsoft) for Windows. Dichotomous data are presented as frequencies. Quantitative data are presented as mean $\pm S D$ and compared using the Student $t$ test, regression analysis, or ANOVA for repeated measures with post hoc testing with the Tukey honestly significant difference test. A probability value of $P<0.05$ was considered significant.

\section{Results}

Demographics, Lipid Profile, and Medication

Demographics, lipid profile, and medication of the study population are presented in Table 1.

\section{Estimated Risk of Clinical Events and Serial IVUS Data}

For the 3 risk algorithms (PROCAM, SCORE, and Framingham), the changes in P\&M, lumen, and EEM CSA for patients with $<10 \%, 10 \%$ to $20 \%$, and $>20 \%$ risk of clinical events are given in Tables 2 through 4 . For all 3 risk algorithms, patients at highest estimated risk showed the greatest IVUS plaque progression. 
Table 1. Patient Demographics, Lipid Status, and Medications.

\begin{tabular}{|c|c|}
\hline & All Patients $(n=56)$ \\
\hline Age, y & $57 \pm 8$ \\
\hline IVUS follow-up, mo & $17.7 \pm 9.4$ \\
\hline Male gender & $50(89)$ \\
\hline Total cholesterol, mg/dL & $199 \pm 39$ \\
\hline LDL cholesterol, mg/dL & $125 \pm 43$ \\
\hline HDL cholesterol, mg/dL & $48 \pm 14$ \\
\hline Triglycerides, mg/dL & $135 \pm 76$ \\
\hline Systolic blood pressure, $\mathrm{mm} \mathrm{Hg}$ & $137 \pm 33$ \\
\hline Diabetes mellitus & $10(18)$ \\
\hline Smoker & $16(29)$ \\
\hline Family history of coronary disease & $10(18)$ \\
\hline Body mass index, $\mathrm{kg} / \mathrm{m}^{2}$ & $26.7 \pm 3.3$ \\
\hline Previous myocardial infarction & $17(30)$ \\
\hline \multicolumn{2}{|l|}{ No. of diseased vessels } \\
\hline 1-Vessel disease & $27(48)$ \\
\hline 2-Vessel disease & $14(25)$ \\
\hline 3-Vessel disease & $15(27)$ \\
\hline \multicolumn{2}{|l|}{ Clinical syndrome } \\
\hline Stable angina CCS class I & $16(29)$ \\
\hline Stable angina CCS class II & $27(48)$ \\
\hline Stable angina CCS class III & $8(14)$ \\
\hline Unstable angina & $5(9)$ \\
\hline \multicolumn{2}{|l|}{ Medication } \\
\hline Acetylsalicylic acid & $56(100)$ \\
\hline ACE inhibitors & $21(38)$ \\
\hline AT1-receptor antagonists & $1(2)$ \\
\hline B-Blockers & $33(59)$ \\
\hline Calcium channel blockers & $17(30)$ \\
\hline Diuretics & $17(30)$ \\
\hline Fibrates & $2(4)$ \\
\hline Insulin & $2(4)$ \\
\hline Nitrates & $36(64)$ \\
\hline Oral antidiabetics & $3(5)$ \\
\hline Statins & $47(84)$ \\
\hline
\end{tabular}

Values are $n(\%)$ or mean $\pm S D$. CCS indicates Canadian Cardiovascular Society. 
Table 2. Serial IVUS Measurements Compared to Estimated Risk of Events Using the PROCAM Algorithm.

\begin{tabular}{|c|c|c|c|c|c|c|c|}
\hline & $\begin{array}{c}<10 \% \text { Risk } \\
\text { of Events in } \\
\text { PROCAM } \\
(n=32)\end{array}$ & $\begin{array}{c}10 \%-20 \% \text { Risk } \\
\text { of Events in } \\
\text { PROCAM } \\
(n=13)\end{array}$ & $\begin{array}{c}>20 \% \text { Risk } \\
\text { of Events in } \\
\text { PROCAM } \\
(n=11)\end{array}$ & ANOVA & $P 1$ & $P 2$ & $P 3$ \\
\hline $\begin{array}{l}\triangle \mathrm{P} \& \mathrm{M} \mathrm{CSA} / \mathrm{y} \\
\mathrm{mm}^{2}\end{array}$ & $-0.1 \pm 0.7$ & $1.2 \pm 0.9$ & $2.3 \pm 1.1$ & $<0.0001$ & $<0.05$ & $<0.01$ & NS \\
\hline $\begin{array}{l}\Delta \mathrm{P} \& \mathrm{M} C S A / y, \\
\%\end{array}$ & $1.0 \pm 6.5$ & $12.7 \pm 10.4$ & $31.5 \pm 17.9$ & $<0.0001$ & NS & $<0.01$ & $<0.01$ \\
\hline $\begin{array}{l}\Delta \text { Lumen CSA/y, } \\
\mathrm{mm}^{2}\end{array}$ & $-0.5 \pm 3.4$ & $-0.2 \pm 2.1$ & $-2.8 \pm 2.5$ & $<0.05$ & NS & $<0.05$ & NS \\
\hline $\begin{array}{l}\Delta \text { Lumen CSA } / y \text {, } \\
\%\end{array}$ & $-0.8 \pm 20.9$ & $-0.6 \pm 16.6$ & $-16.9 \pm 11.3$ & $<0.01$ & NS & $<0.05$ & $<0.05$ \\
\hline $\begin{array}{l}\Delta \mathrm{EEM} C S A / \mathrm{y} \\
\mathrm{mm}^{2}\end{array}$ & $-0.6 \pm 3.6$ & $1.0 \pm 2.0$ & $-0.4 \pm 2.8$ & 0.6 & $\ldots$ & $\ldots$ & $\ldots$ \\
\hline $\begin{array}{l}\Delta \text { EEM CSA } / y, \\
\%\end{array}$ & $-2.1 \pm 11.6$ & $4.8 \pm 8.9$ & $-0.1 \pm 8.9$ & 0.6 & $\ldots$ & $\ldots$ & $\ldots$ \\
\hline
\end{tabular}

Post hoc P 1: <10\% Risk vs 10\%-20\% Risk; P 2: <10\% Risk vs >20\% Risk; $P$ 3: $10 \%-20 \%$ Risk vs $>20 \%$ Risk.

Table 3. Serial IVUS Measurements Compared to Estimated Risk of Events Using the SCORE Algorithm.

\begin{tabular}{|c|c|c|c|c|c|c|c|}
\hline & $\begin{array}{c}<10 \% \text { Risk } \\
\text { of Events in } \\
\text { SCORE } \\
(n=35)\end{array}$ & $\begin{array}{c}10 \%-20 \% \text { Risk } \\
\text { of Events in } \\
\text { SCORE } \\
(n=14)\end{array}$ & $\begin{array}{c}>20 \% \text { Risk } \\
\text { of Events in } \\
\text { SCORE } \\
(n=7)\end{array}$ & ANOVA & $P 1$ & $P 2$ & $P 3$ \\
\hline $\begin{array}{l}\Delta \mathrm{P} \& \mathrm{M} \text { CSA/y } \\
\mathrm{mm}^{2}\end{array}$ & $0.2 \pm 1.4$ & $1.3 \pm 0.7$ & $2.8 \pm 0.9$ & $<0.0001$ & NS & $<0.01$ & $<0.05$ \\
\hline $\begin{array}{l}\Delta \mathrm{P} \& \mathrm{M} C S A / y \\
\%\end{array}$ & $6.1 \pm 18.6$ & $14.0 \pm 9.7$ & $35.7 \pm 15.7$ & $<0.001$ & NS & $<0.01$ & $<0.01$ \\
\hline $\begin{array}{l}\Delta \text { Lumen CSA/y, } \\
\mathrm{mm}^{2}\end{array}$ & $-0.1 \pm 3.2$ & $-0.6 \pm 3.0$ & $-2.5 \pm 1.1$ & 0.18 & $\ldots$ & $\ldots$ & $\ldots$ \\
\hline $\begin{array}{l}\Delta \text { Lumen CSA/y, } \\
\%\end{array}$ & $0.1 \pm 19.1$ & $-1.2 \pm 16.3$ & $-18.4 \pm 9.7$ & $<0.05$ & NS & $<0.05$ & $<0.05$ \\
\hline $\begin{array}{l}\Delta \mathrm{EEM} \mathrm{CSA} / \mathrm{y} \\
\mathrm{mm}^{2}\end{array}$ & $0.1 \pm 3.6$ & $0.7 \pm 3.0$ & $0.4 \pm 1.5$ & 0.9 & $\ldots$ & $\ldots$ & $\ldots$ \\
\hline $\begin{array}{l}\Delta \mathrm{EEM} C S A / y, \\
\%\end{array}$ & $0.8 \pm 13.1$ & $4.1 \pm 10.4$ & $2.7 \pm 6.6$ & 0.7 & $\ldots$ & $\ldots$ & $\ldots$ \\
\hline
\end{tabular}

Post hoc P 1: <10\% Risk vs 10\%-20\% Risk; P 2: <10\% Risk vs >20\% Risk; P 3: $10 \%-20 \%$ Risk vs $>20 \%$ Risk. 
Table 4. Serial IVUS Measurements Compared to Estimated Risk of Events Using the Framingham Algorithm.

\begin{tabular}{|c|c|c|c|c|c|c|c|}
\hline & $\begin{array}{c}<10 \% \text { Risk } \\
\text { of Events in } \\
\text { Framingham } \\
(n=15)\end{array}$ & $\begin{array}{c}10 \%-20 \% \text { Risk } \\
\text { of Events in } \\
\text { Framingham } \\
(n=32)\end{array}$ & $\begin{array}{c}>20 \% \text { Risk } \\
\text { of Events in } \\
\text { Framingham } \\
\quad(n=9)\end{array}$ & ANOVA & $P 1$ & $P 2$ & $P 3$ \\
\hline $\begin{array}{l}\triangle \mathrm{P} \& \mathrm{M} \mathrm{CSA} / \mathrm{y}, \\
\mathrm{mm}^{2}\end{array}$ & $-0.1 \pm 0.7$ & $0.9 \pm 1.7$ & $1.8 \pm 0.9$ & $<0.01$ & NS & $<0.01$ & NS \\
\hline$\underset{\%}{\Delta \mathrm{P} \& \mathrm{M} C S A / y}$ & $1.0 \pm 6.5$ & $14.7 \pm 22.2$ & $19.5 \pm 12.7$ & $<0.05$ & NS & $<0.05$ & NS \\
\hline $\begin{array}{l}\Delta \text { Lumen CSA/y, } \\
\mathrm{mm}^{2}\end{array}$ & $-0.5 \pm 3.4$ & $-0.2 \pm 2.7$ & $-1.8 \pm 3.3$ & 0.4 & $\ldots$ & $\ldots$ & $\ldots$ \\
\hline $\begin{array}{l}\Delta \text { LumenCSA } / y \text {, } \\
\%\end{array}$ & $-0.8 \pm 20.9$ & $-1.5 \pm 17.9$ & $-9.3 \pm 15.5$ & 0.5 & $\ldots$ & $\ldots$ & $\ldots$ \\
\hline $\begin{array}{l}\Delta \mathrm{EEM} C S A / y \\
\mathrm{~mm}^{2}\end{array}$ & $-0.6 \pm 3.6$ & $0.7 \pm 3.0$ & $0.1 \pm 3.3$ & 0.4 & $\ldots$ & $\ldots$ & $\ldots$ \\
\hline $\begin{array}{l}\Delta \text { EEM CSA } / y, \\
\%\end{array}$ & $-2.1 \pm 11.6$ & $3.5 \pm 12.1$ & $2.3 \pm 10.6$ & 0.3 & $\ldots$ & $\ldots$ & $\ldots$ \\
\hline
\end{tabular}

Individual Risk Factors, Medication, and Plaque Progression

Annual changes in P\&M CSA of patients with high versus low values of individual risk factors are shown in Figure 1; P\&M CSA progression was significantly greater for smokers and for patients with unfavorable serum cholesterol profile. When studied as continuous variables, annual changes in P\&M CSA correlated with LDL and $\mathrm{HDL}$ cholesterol as previously reported ${ }^{6}$; with total cholesterol $(r=0.27, P<0.05)$ and total cholesterol/HDL ratio $(r=0.43, P<0.001)$ but not with triglycerides $(r=-0.02$, $P=0.9)$; and not with age $(r=0.06, P=0.7)$ or systolic blood pressure $(r=0.07, P=0.6)$. Statin use $(n=47)$ was associated with less P\&M CSA progression $(11.2 \pm 19.6 \%$ versus $24.2 \pm 18.6 \%, P<0.05)$; other drugs had no impact. 


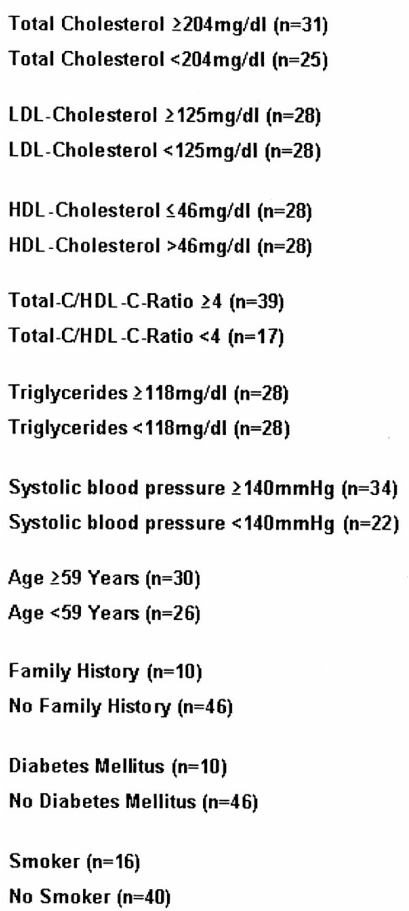

\section{Plaque \& Media Changes / Year (\%)}

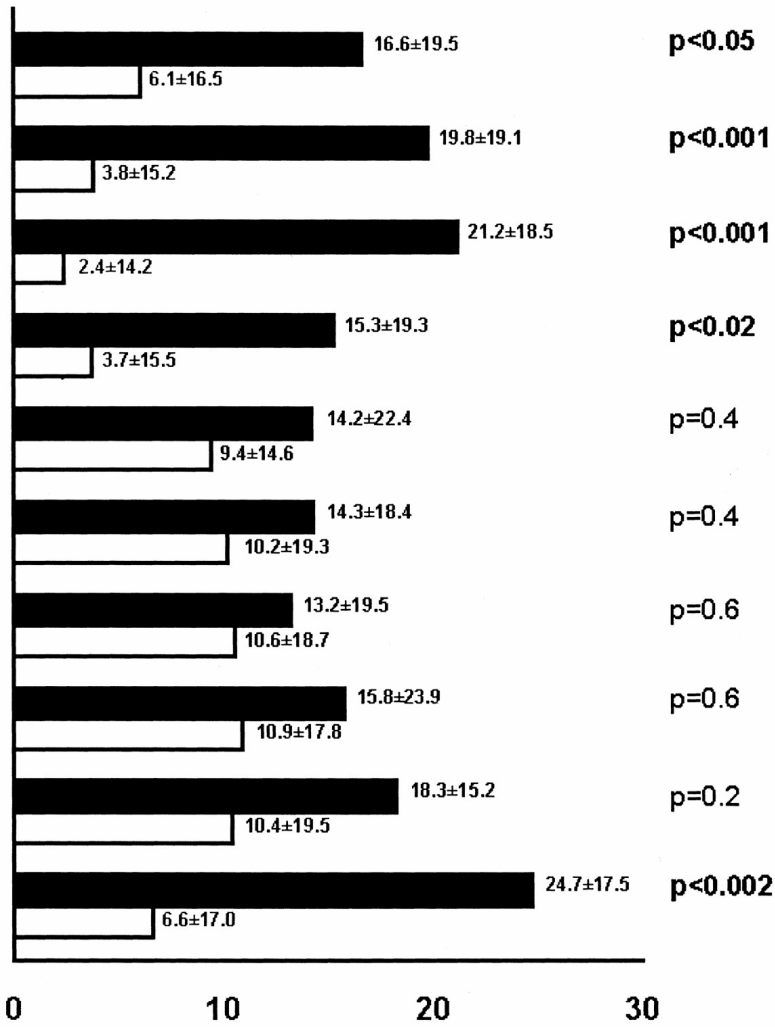

Figure 1. Annual P\&M CSA progression in subgroups.

\section{Estimated Risk Versus Changes in Plaque and Lumen Dimensions}

The relation between changes in P\&M CSA and lumen CSA versus estimated risk of events is shown in Figure 2. There was a linear relation between P\&M CSA progression versus estimated risk of clinical events and between lumen CSA decrease versus estimated risk of clinical events. 

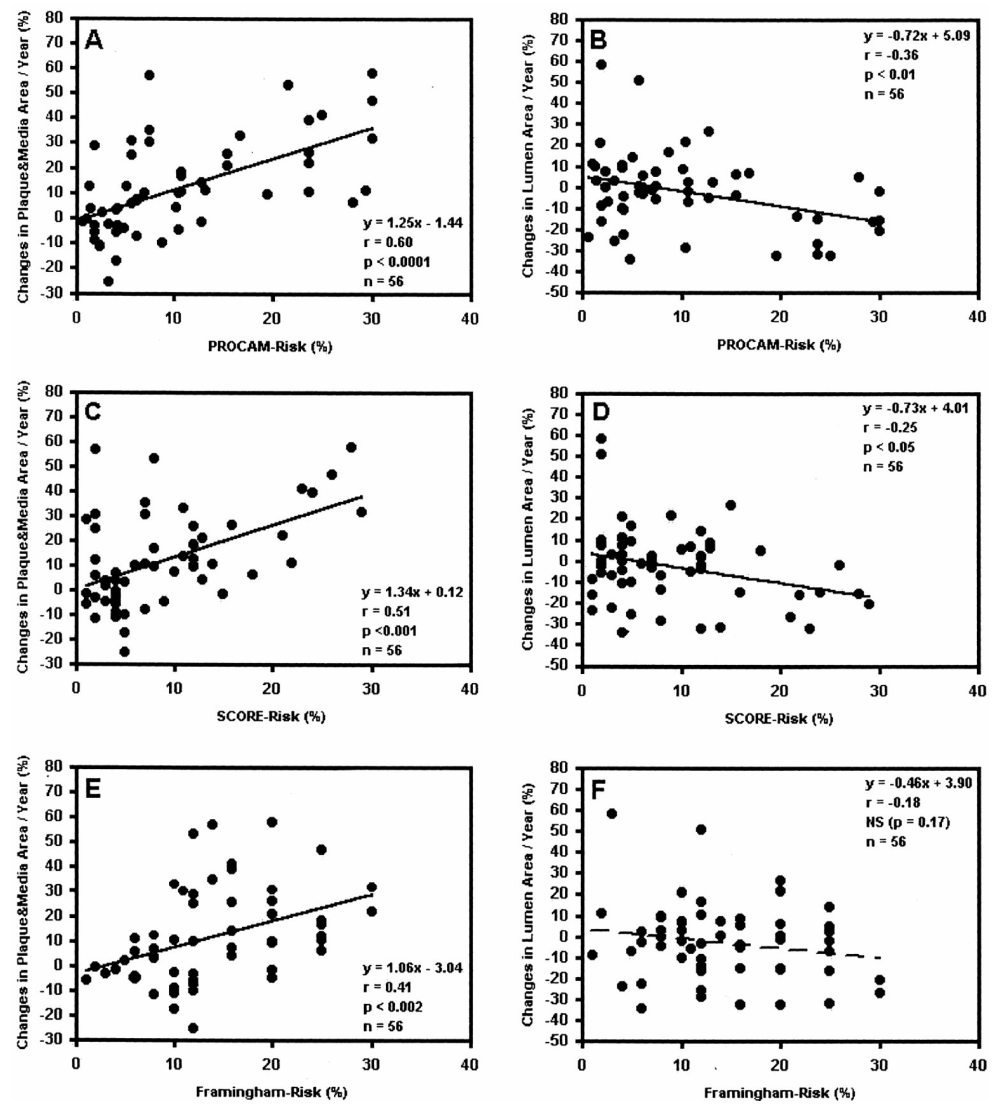

Figure 2. PROCAM risk (A, B), SCORE risk (C, D), and Framingham risk $(E, F)$ versus changes in P\&M CSA (left) and changes in lumen CSA (right).

\section{Plaque Progression and Actual Adverse Cardiovascular Events}

During follow-up, 5 patients had an acute myocardial infarction; 7, unstable angina; and 6 , percutaneous interventions of new de novo coronary lesions. Figure 3 presents annual P\&M CSA changes together with information on adverse events for each patient. Patients with adverse events $(n=18)$ had more annual P\&M CSA progression than the rest of the population $(25.2 \pm 19.4 \%$ versus $5.9 \pm 15.6 \%$, $P<0.001)$ but showed no significant difference in length of follow-up. Similarly, patients with myocardial infarction $(n=5)$ as well as patients with myocardial infarction or unstable angina $(n=12)$ had more annual P\&M CSA progression than the rest of the population $(30.9 \pm 7.4 \%$ versus $9.9 \pm 18.7 \%, P<0.001$, and $29.8 \pm 15.3 \%$ versus $6.9 \pm 16.8 \%, P<0.0003$, respectively). 


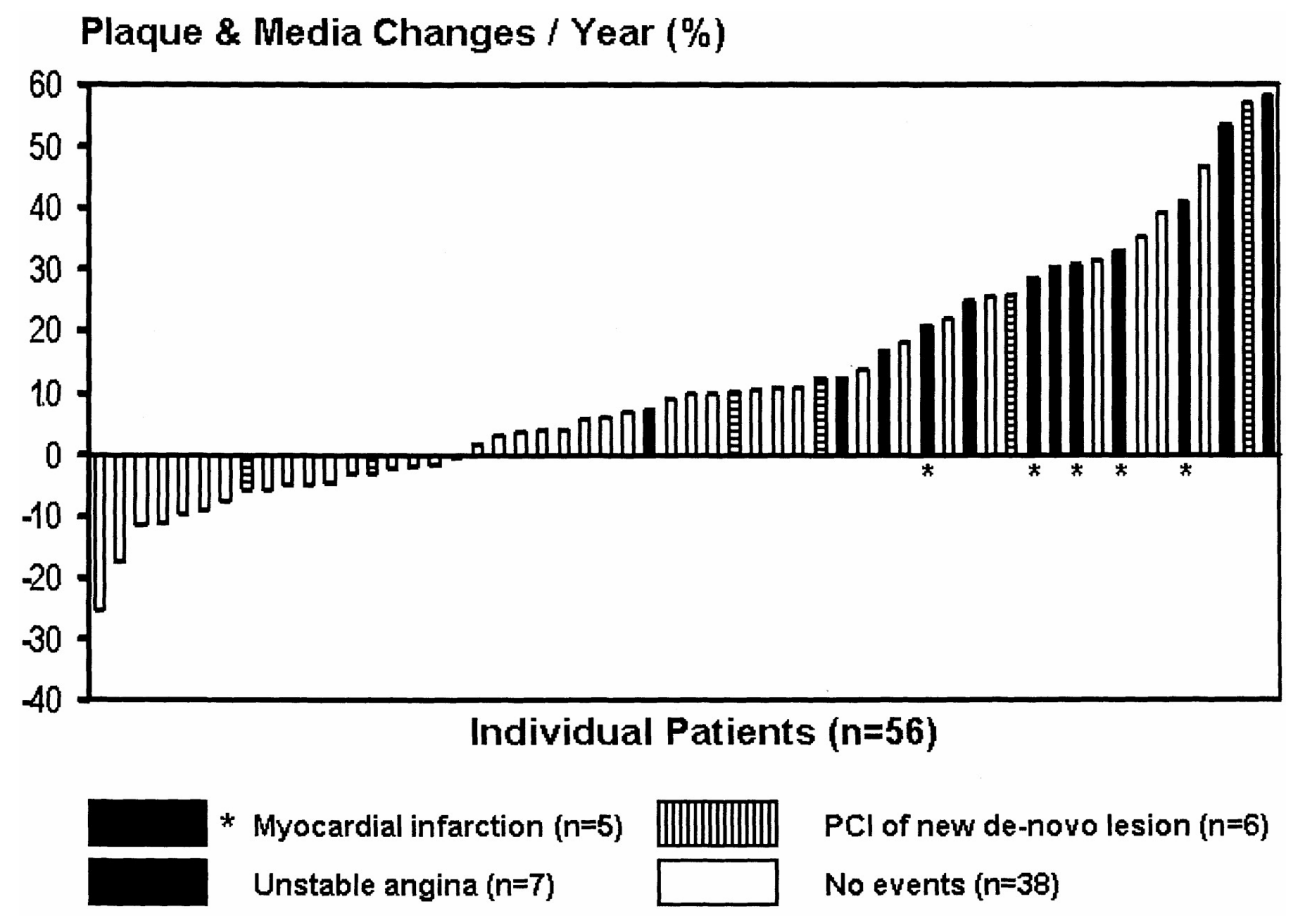

Figure 3. Annual changes in P\&M CSA and adverse cardiovascular events during follow-up period.

\section{Discussion}

This study showed a positive linear relationship between estimated risk of clinical events derived from all 3 established risk scores and the extent of plaque progression as measured with serial IVUS. This translated into a greater decrease in lumen dimensions with increasing risk of clinical events. For all 3 algorithms, patients with the highest estimated risk of clinical events showed the greatest plaque progression by IVUS. Risk prediction by PROCAM score showed the strongest correlation with serial IVUS measurements.

Our findings are of interest because there is an increasing use of IVUS as an end point of pharmacological interventions designed to limit progression or induce regression of coronary artery disease..$^{2-6}$ However, despite the present results, there is a lack of data linking changes in plaque dimensions, as measured with IVUS, with clinical outcome. ${ }^{5}$ Thus, there is a need for future prospective trials to 
combine serial IVUS assessment of coronary plaque progression with long-term clinical follow-up.

\section{Risk Assessment in Patients With Established Coronary Heart Disease}

In this study, we compared plaque progression by serial IVUS in patients with established coronary artery disease, a secondary measure, with established risk scores for primary disease prevention. We consider this approach to be reasonable in the absence of a validated risk score for secondary prevention. Although the major risk factors that are important in primary prevention remain important in secondary prevention ${ }^{12,24-26}$, all individuals examined in the present study have an absolute risk of clinical events that was higher than suggested by the nominal results of the 3 algorithms. Nevertheless, we were able to demonstrate with all 3 algorithms a linear relationship between estimated risk of clinical events and the extent of atherosclerotic plaque progression as measured with serial IVUS.

\section{Risk Scores}

The risk-scoring methods studied in the present report were derived from the European SCORE Project ${ }^{16}$, the German PROCAM Study ${ }^{18}$, and the US Framingham Heart and Offspring Studies. ${ }^{19}$ In fact, the PROCAM Study was performed in the city of Münster; Münster is located next to Essen, the city in which the present IVUS study was performed. Therefore, perhaps not surprisingly, we observed a particularly strong relationship between plaque progression and estimated risk of clinical events using the PROCAM score. Conversely, the correlation of the SCORE algorithm and (even more) of the Framingham risk score showed relationships with the current IVUS data that were less strong but still significant. This suggests that risk scores derived from population studies may have a somewhat limited applicability when applied to another population with a different risk profile from a different geographical region, even if both populations were Western communities. ${ }^{27}$ This may be even more of a problem when the 2 populations are from very different cultures and genetic makeups, for example, applying Western scores to Asian patients.

Of note, SCORE, PROCAM, and Framingham algorithms used different end points (risk of cardiac events versus cardiovascular mortality; fatal versus combined fatal and nonfatal end points); this makes direct comparison difficult. Nevertheless, our findings were quite similar among the 3 risk scores.

Our data suggest that compared with conventional studies of cardiovascular risk, IVUS assessment of plaque and lumen dimensions may provide valuable insights from relatively small study populations within a relatively short period of 
time. This article may be a first step to applying direct assessment of plaque progression or regression by IVUS to fine-tune our understanding of cardiovascular risk factors. As a consequence, IVUS assessment of coronary atherosclerosis may be used to develop and/or validate secondary risk factor scores in the future.

\section{Limitations}

By most standards, this was a large serial IVUS study; however, all studies with long-term serial IVUS assessment of atherosclerosis are limited to a relatively small number of patients. We were able to include only patients with significant coronary artery disease who were admitted for repeat cardiac catheterization $\geq 12$ months after baseline. Because of this selection bias, the findings of the present study may not be applicable to a population of patients treated by a single percutaneous coronary intervention or even to the general population. We used 2 IVUS systems in the present study; although this approach may have minor shortcomings, every effort was made to obtain the most reliable data possible, as discussed in detail previously. ${ }^{6}$ Three-dimensional IVUS analysis may be superior for the assessment of progression/regression. The patients in the present study had established coronary atherosclerosis. We studied only left main disease as representative of segments that did not receive intervention.

\section{Conclusions}

There is a positive linear relation between the estimated risk of clinical events derived from 3 established risk scores (that were initially developed to predict primary events) and the extent of plaque progression as measured with serial IVUS in patients with established coronary artery disease. For all 3 algorithms, patients at highest estimated risk showed the greatest plaque progression by IVUS. This translated into the greatest reduction in lumen dimensions in patients with the highest risk of events.

\section{References}

1. Mintz GS, Nissen SE, Anderson WD, et al. American College of Cardiology clinical expert consensus document on standards for acquisition, measurement and reporting of intravascular ultrasound studies (IVUS). J Am Coll Cardiol 2001;37:1478-1492.

2. Takagi T, Yoshida K, Akasaka T, et al. Intravascular ultrasound analysis of reduction in progression of coronary narrowing by treatment with pravastatin. Am J Cardiol 1997;79:1673-1676.

3. Shiran A, Mintz GS, Leiboff $B$, et al. Serial volumetric intravascular ultrasound assessment of arterial remodeling in left main coronary artery disease. Am J Cardiol 1999;83:1427-1432. 
4. Schartl M, Bocksch W, Koschyk DH, et al. for the GAIN-Study Investigators. Use of intravascular ultrasound to compare effects of different strategies of lipid-lowering therapy on plaque volume and composition in patients with coronary artery disease. Circulation 2001;104:387-392.

5. Nissen SE, Tsunoda T, Tuzcu EM, et al. Effect of recombinant apoA-I Milano on coronary atherosclerosis in patients with acute coronary syndromes. JAMA 2003;290:2292-2300.

6. von Birgelen C, Hartmann M, Mintz GS, et al. Relation between progression and regression of atherosclerotic left main coronary artery disease and serum cholesterol levels as assessed with serial long-term ( $\geq 12$ months) follow-up intravascular ultrasound. Circulation 2003;108:2757-2762.

7. Scandinavian Simvastatin Survival Study Group. Randomized trial of cholesterol lowering in 4444 patients with coronary heart disease: the Scandinavian Simvastatin Survival Study (4S). Lancet 1994;344:1383-1389.

8. Shepherd J, Cobbe SM, Ford I, et al. for the West of Scotland Coronary Prevention Study Group. Prevention of coronary artery disease with pravastatin in men with hypercholesterolemia. N Engl J Med 1995;333:1301-1307.

9. Sacks FM, Pfeffer MA, Moye LA, et al. for the Cholesterol And Recurrent Events (CARE) trial investigators. The effect of pravastatin on coronary events after myocardial infarction in patients with average cholesterol levels. N Engl J Med 1996;335:1001-1009.

10. Downs JR, Clearfield M, Weis $\mathrm{S}$, et al. Primary prevention of acute coronary events with lovastatin in men and women with average cholesterol levels: results of AFCAPS/TexCAPS. Air Force/Texas Coronary Atherosclerosis Prevention Study. JAMA 1998;279:1615-1622.

11. Ballantyne $\mathrm{CM}$. Clinical trials endpoints: angiograms, events and plaque instability. Am J Cardiol 1998;82:5M-11M.

12. Wood D, De Backer G, Faergeman O, et al. Prevention of coronary heart disease in clinical practice: recommendations of the second joint task force of European and other societies on coronary prevention. Atherosclerosis 1998;140:199-270.

13. Grundy SM, Pasternak R, Greenland P, et al. Assessment of cardiovascular risk by use of multiplerisk-factor assessment equations: a statement for healthcare professionals from the American Heart Association and the American College of Cardiology. Circulation 1999;100:1481-1492.

14. Fodor JG, Frohlich JJ, Genest JJ Jr, et al. Recommendations for the management and treatment of dyslipidemia: report of the working group on hypercholesterolemia and other dyslipidemias. CMAJ 2000;162:1441-1447.

15. Expert Panel on Detection, Evaluation, and Treatment of High Blood Cholesterol in Adults. Executive summary of the third report of the National Cholesterol Education Program (NCEP) expert panel on detection, evaluation, and treatment of high blood cholesterol in adults (Adult Treatment Panel III). JAMA 2001;285:2486-2497.

16. Conroy RM, Pyörälä K, Fitzgerald AP, et al, for the SCORE project group. Estimation of ten-year risk of fatal cardiovascular disease in Europe: the SCORE project. Eur Heart J 2003;24:987-1003.

17. De Backer G, Ambrosioni E, Borch-Johnsen K, et al. European guidelines on cardiovascular disease prevention in clinical practice. Eur Heart J 2003;24:1601-1610.

18. Assmann G, Cullen P, Schulte H. Simple scoring scheme for calculating the risk of acute coronary events based on the 10-year follow-up of the Prospective Cardiovascular Münster (PROCAM) Study [published correction appears in Circulation 2002;105:900]. Circulation 2002;105:310-315.

19. Anderson KM, Wilson PW, Odell PM, et al. An updated coronary risk profile: a statement for health professionals. Circulation 1991;83:356-362.

20. Assmann G, Carmena R, Cullen P, et al. Coronary heart disease: reducing the risk: a worldwide view. International Task Force for the Prevention of Coronary Heart Disease. Circulation 1999;100: 1930-1938.

21. Baumgart $D$, Haude $M, G e J$, et al. Online integration of intravascular ultrasound images into angiographic images. Cathet Cardiovasc Diagn 1996;39:328-329. 
22. von Birgelen C, Airiian SG, Mintz GS, et al. Variations of remodeling in response to left main atherosclerosis assessed with intravascular ultrasound in vivo. Am J Cardiol 1997;80:1408-1413.

23. von Birgelen C, Klinkhart W, Mintz GS, et al. Plaque distribution and vascular remodeling of ruptured and nonruptured coronary plaques in the same vessel: an intravascular ultrasound study in vivo. J Am Coll Cardiol 2001;37:1864-1870.

24. Smith SC Jr, Blair SN, Criqui $\mathrm{MH}$, et al. Preventing heart attack and death in patients with coronary disease. Circulation 1995;92:2-4.

25. Grover SA, Paquet S, Levinton C, et al. Estimating the benefits of modifying risk factors of cardiovascular disease: a comparison of primary vs secondary prevention [published correction appears in Arch Intern Med. 1998;158:1228]. Arch Intern Med 1998;158:655-662.

26. Genest J, Pedersen TR. Prevention of cardiovascular ischemic events: high-risk and secondary prevention. Circulation 2003;107:2059-2065.

27. Hense HW, Schulte $H$, Lowel $H$, et al. Framingham risk function overestimates risk of coronary heart disease in men and women from Germany: results from MONICA Augsburg and the PROCAM cohorts. Eur Heart J 2003;24:1-9. 



\section{Chapter 6}

Spectrum of Remodeling Behavior Observed With

Serial Long-Term ( $\geq 12$ Months) Follow-Up

Intravascular Ultrasound Studies in Left Main

Coronary Arteries

Clemens von Birgelen, Marc Hartmann, Gary S. Mintz, Dirk Böse, Holger Eggebrecht, Mario Gössl, Till Neumann, Dietrich Baumgart, Heinrich Wieneke, Axel Schmermund, Michael Haude, Raimund Erbel

Reprinted with permission from:

The American Journal of Cardiology 2004;93(9):1107-1113.

(c) 2004 Elsevier Inc. 



\section{Abstract}

Most intravascular ultrasound (IVUS) studies of arterial remodeling in native coronary arteries reported a remodeling index obtained at a single time point.

We analyzed serial IVUS examinations, including the vessel cross-sectional area changes (remodeling behavior), of 60 hemodynamically nonstenotic left main lesions (baseline vs $18.4 \pm 9.4$ months follow-up). Lumen reduction resulted from vessel reduction (sometimes despite plaque+media decrease), plaque+media increase (with or without vessel increase), or both. The percent annual changes in lumen area correlated strongly with changes in vessel $(r=0.84)$, but not with changes in plaque+media area. Plaques were classified as group A lesions, reflecting positive remodeling behavior (vessel changes $>0$ ), or group B lesions, reflecting negative (or intermediate) remodeling behavior (vessel changes $\leq 0$ ). Both groups did not differ significantly in demographics, laboratory data, and medications. Group A lesions $(n=40)$ more often showed plaque+media increase than group B lesions (32 of 40 [80\%] vs 9 of 20 [45\%]; $p=0.02$ ). Group A lesions had, on average, mild annual lumen increase despite mild plaque+media increase, i.e, overcompensation of remodeling for plaque+media increase (vessel increase greater than plaque+media area increase, 19 of $40[47 \%])$. Conversely, group B lesions $(n=20)$ showed a significant lumen area reduction $\left(-2.8 \pm 2.6 \mathrm{~mm}^{2} /\right.$ year) as a result of a decrease in vessel area only.

Thus, serial long-term reduction of lumen size may result from vessel shrinkage (sometimes despite plaque decrease), plaque increase (with or without vessel increase), or both; overall, only the remodeling behavior has a significant relation to lumen changes. More than $30 \%$ of lesions show a negative remodeling behavior, which shows no relation to patient characteristics or initial plaque burden.

\section{Introduction}

Atherosclerotic coronary arteries undergo expansive remodeling to accommodate an increasing plaque burden, especially during early stages of plaque accumulation. ${ }^{1}$ Conversely, failure of expansive remodeling and even constrictive remodeling (i.e., "shrinkage") may contribute to development of significant stenoses. $^{2-8}$ Intravascular ultrasound (IVUS) permits transmural visualization of arteries; this allows assessment of arterial remodeling in vivo. ${ }^{9-20}$ However, most previous IVUS studies of arterial remodeling compared the lesion site with the proximal and/or distal reference segments at a single time point rather than over the natural history of the disease process. ${ }^{20}$ As such, these studies assessed remodeling patterns rather than the natural history of the remodeling process (i.e., the remodeling behavior). Published data contain only a few serial IVUS studies of native nontransplanted coronary arteries, most with follow-up of 6 to 12 months. ${ }^{21-}$ ${ }^{24}$ Little is known about the serial changes of plaque and lumen dimensions and of other atherosclerotic lesion morphologies, especially when compared with serial assessment of the remodeling process. We therefore analyzed serial IVUS data of nonstenotic left main coronary arteries at baseline and during $\geq 12$ months of followup. 


\section{Methods}

\section{Study group}

We analyzed serial IVUS examinations of 60 hemodynamically nonsignificant de novo atherosclerotic plaques in left main coronary arteries during a follow-up period of $\geq 12$ months (mean 18.4 \pm 9.4 ). ${ }^{24}$ These plaques were identified from our database and met the following criteria: (1) serial high-quality IVUS imaging of the entire left main stem $\geq 12$ months apart; (2) calcifications that did not limit the quantitative assessment of vessel cross-sectional area (shadowing $\leq 75^{\circ}$ of the adventitial circumference by individual calcific deposits); (3) nonostial target site location; (4) angiographic lumen diameter stenosis $<30 \%$ ("worst view" visual assessment); and (5) no intervention in the very proximal segments of the left anterior descending or circumflex coronary arteries because these interventions could have affected the left main artery. All patients were examined in the Essen University Cardiac Catheterization Laboratory. The IVUS study was approved by the local council on human research, and all patients signed a written informed consent form as approved by the local medical ethics committee.

Cardiovascular risk factors, clinical data, and medication

In our laboratory, we prospectively record demographics, cardiovascular risk factors, medications, and results of key laboratory tests of patients examined with IVUS. All laboratory tests were performed at baseline and follow-up as part of the clinical routine, and were analyzed in the central laboratory of Essen University according to international standards. Cardiovascular risk factors that were recorded included diabetes mellitus and hypertension (both medication-dependent only), hypercholesterolemia (medication-dependent, total serum cholesterol $>200 \mathrm{mg} / \mathrm{dl}$, or low-density lipoprotein cholesterol $>160 \mathrm{mg} / \mathrm{dl}$ ), history of smoking, and family history of coronary artery disease. Data of the laboratory tests were the mean of the 2 values (at baseline and follow-up); medication was recorded only if a drug was taken for $>50 \%$ of the follow-up interval (e.g., clopidogrel for 4 weeks was not tabulated).

\section{IVUS imaging protocol}

IVUS imaging was initially performed during percutaneous coronary interventions of mid- or distal left anterior or left circumflex arteries. IVUS studies were performed after intracoronary injections of $200 \mu \mathrm{g}$ of nitroglycerin with 2 commercially available systems. The first IVUS system ( $n=29$ cases) was a 
mechanical sector scanner (Boston Scientific Corporation, San Jose, California) incorporating a $30-\mathrm{MHz}$, single-element, beveled transducer rotating at 1,800 rpm; the second system ( $n=31$ cases) was a solid-state device (Endosonics, Rancho Cordova, California). Importantly, at Essen University, if a patient undergoes imaging with 1 IVUS system during an index procedure, the same IVUS system is used at follow-up. Slow continuous pullbacks of the IVUS transducer were begun as distal as possible in one of the left coronary arteries and were generally performed using a motorized pull-back device. IVUS images of the entire pullback were recorded on 0.5-inch high-resolution s-VHS tape for off-line analysis. In addition, a dedicated image-in-image system (Echo-Map, Siemens, Erlangen, Germany) $)^{25}$ was used to record the "angiographic" position of the IVUS probe together with the corresponding IVUS image, especially at sites of characteristic landmarks (i.e., calcifications or unusual plaque shapes) or the target site, or both. Follow-up IVUS studies were performed (generally using the same IVUS system as was initially used) during repeat coronary interventions ( $n=34,57 \%)$, and during IVUS examinations of ambiguous left coronary lesions or (clinically driven) followup catheterizations $(n=26,43 \%)$. IVUS was not performed as part of another study with long-term IVUS follow-up in any patients. There were no complications related to IVUS.

\section{Quantitative IVUS analysis}

The target site image slice was determined from the initial IVUS study; this was the site with the smallest lumen area. If there were several slices with equal lumen size, the one with the largest vessel and plaque+media (plaque+media = vessel minus lumen) cross-sectional area was analyzed. ${ }^{20}$ Exact matching of the target site on the initial and follow-up IVUS studies was ensured using side-by-side comparison of the serial IVUS video sequences along with information of the pullback speed, the operators' recorded comments (on videotape), and characteristic calcifications, vascular and perivascular landmarks, and plaque shapes. ${ }^{24}$ If required, $x$-ray sequences of the dedicated image-in-image system (Echo-Map) were revisited to optimize matching. ${ }^{24,25}$ The lumen cross-sectional area was measured by tracing the leading edge of the intima. The vessel cross-sectional area was measured by tracing the leading edge of the adventitia. As in many previous IVUS studies, plaque+media was used as a measure of atherosclerotic plaque because IVUS cannot measure media thickness accurately. In our laboratory, the intraclass correlation coefficient is 0.99 for repeated measurements of vessel area, 0.96 for lumen, and 0.99 for plaque+media cross-sectional area. Plaque burden (\%) was calculated as (plaque+media divided by vessel cross-sectional area) $\times 100 \%$. To compensate for the variation in follow-up interval and to obtain comparable data, 
we calculated the absolute and relative changes between the initial and follow-up IVUS data; measurements were normalized for the length of the follow-up period (changes per year) and for baseline measurements.

\section{Arterial remodeling}

The changes in vessel area were used as a measure of serial arterial remodeling (i.e., arterial remodeling behavior). This way of assessing arterial remodeling differs substantially from the remodeling index that was calculated in various previous nonserial IVUS studies in which the lesion site vessel area is divided by the reference vessel area. Remodeling behavior in the present study is independent of the reference segments, and reflects the true changes in vessel dimensions in relation to the change in plaque dimensions. These values of changes in vessel area were used to divide patients into those with positive arterial remodeling behavior (changes in vessel area $>0$, group A) versus those with intermediate or negative arterial remodeling behavior (i.e., arterial "shrinkage" [changes in vessel area $\leq 0$ ], group B). In addition, some group A plaques reflected overcompensation by positive arterial remodeling; these plaques had changes in vessel area that were greater than plaque+media increase.

\section{IVUS assessment of plaque composition}

IVUS images were read off-line by 3 experienced cardiologists. Plaque composition was assessed visually as previously described ${ }^{20}$; predominant plaque composition was classified as "soft," "fibrous," "mixed," or calcified. ${ }^{20}$ The arc of target lesion calcium (degree) was measured using a protractor centered on the lumen; if necessary, the total arc of calcium was obtained by adding up arcs of individual deposits. Plaques were classified as calcified if the total arc of lesion calcium was $>180^{\circ}$. Extrapolation of the vessel boundary behind calcium was possible if each individual calcific deposit did not shadow $>75^{\circ}$ of the adventitial circumference.

\section{Statistical analysis}

Analyses were performed with SPSS version 10.0.7 (SPSS Inc., Chicago, Illinois) and MedCalc (version 4.16, Mariakerke, Belgium) software packages for Windows (Microsoft, Redmond, Washington). Dichotomous data are presented as frequencies and compared using chi-square statistics, Fisher's exact test, or Cochrane-Mantel-Haenszel's test for general association. Quantitative data are presented as mean $\pm 1 S D$ and compared using Student's $t$ test and linear regression analysis. A $p$ value $<0.05$ was considered significant. 


\section{Results}

Demographics, medication, and laboratory testing

Demographics (Table 1) and medical treatment and results of laboratory tests (Table 2) did not differ significantly between group A lesions (those with positive serial arterial remodeling) and group B lesions (those with negative or no serial arterial remodeling)

Remodeling and baseline IVUS data

In 40 plaques, changes in vessel area were $>0$ (group $A$ ), and in 20 plaques changes in vessel area were $\leq 0$ (group $B$ ). Baseline plaque burden and plaque cross-sectional area were similar between the 2 groups, whereas both vessel area and lumen area were larger in group B lesions $(p<0.05$ for both, respectively, Table 3). There was no significant differences in plaque composition between the groups. 
Table 1. Patient Demographics

\begin{tabular}{|c|c|c|c|}
\hline & $\begin{array}{c}\text { Group A } \\
\text { (vessel area } \\
\text { changes }>0) \\
(n=40)\end{array}$ & $\begin{array}{c}\text { Group B } \\
\text { (vessel area } \\
\text { changes } \leq 0) \\
(n=20)\end{array}$ & $\mathbf{p}$ \\
\hline Time of follow-up (mo) & $17.2 \pm 7.0$ & $20.4 \pm 12.8$ & 0.3 \\
\hline Age (yrs) & $58 \pm 10$ & $58 \pm 6$ & 0.6 \\
\hline Men & $34(85 \%)$ & $16(80 \%)$ & 0.7 \\
\hline Body mass index $\left(\mathrm{kg} / \mathrm{m}^{2}\right)$ & $26.7 \pm 3.5$ & $26.7 \pm 2.7$ & 0.8 \\
\hline Previous myocardial infarction & $13(33 \%)$ & $8(40 \%)$ & 0.8 \\
\hline Hypercholesterolemia & $18(45 \%)$ & $10(50 \%)$ & 0.9 \\
\hline Systemic arterial hypertension & $29(73 \%)$ & $14(70 \%)$ & 0.9 \\
\hline Diabetes Mellitus & $6(15 \%)$ & $2(10 \%)$ & 0.7 \\
\hline Smoker & $11(28 \%)$ & $5(25 \%)$ & 0.9 \\
\hline Family history of coronary disease & $10(25 \%)$ & $4(20 \%)$ & 0.8 \\
\hline 1 vessel narrowed $>50 \%$ & $16(40 \%)$ & $12(60 \%)$ & \\
\hline 2 vessels narrowed $>50 \%$ & $13(32 \%)$ & $3(15 \%)$ & \\
\hline 3 vessels narrowed $>50 \%$ & $11(28 \%)$ & $5(25 \%)$ & 0.3 \\
\hline Stable angina CCS class I & $12(30 \%)$ & $5(25 \%)$ & \\
\hline Stable angina CCS class II & $18(45 \%)$ & $11(55 \%)$ & \\
\hline Stable angina CCS class III & $6(15 \%)$ & $3(15 \%)$ & \\
\hline Unstable angina & $4(10 \%)$ & $1(5 \%)$ & 0.9 \\
\hline
\end{tabular}

CCS = Canadian Cardiovascular Society 
Table 2. Medications and Laboratory Tests

\begin{tabular}{|c|c|c|c|}
\hline & $\begin{array}{c}\text { Group A } \\
\text { (vessel area } \\
\text { changes }>0) \\
(n=40)\end{array}$ & $\begin{array}{c}\text { Group B } \\
\text { (vessel area } \\
\text { changes } \leq 0) \\
(n=20)\end{array}$ & p \\
\hline \multicolumn{4}{|l|}{ Medication } \\
\hline Acetylsalicylic acid & $40(100 \%)$ & $20(100 \%)$ & 1.0 \\
\hline ACE inhibitors & $14(35 \%)$ & $8(40 \%)$ & 0.9 \\
\hline Angiotensin receptor antagonists & $1(3 \%)$ & $1(5 \%)$ & 0.6 \\
\hline ß blockers & $21(53 \%)$ & $15(75 \%)$ & 0.2 \\
\hline Calcium channel blockers & $13(33 \%)$ & $4(20 \%)$ & 0.4 \\
\hline Diuretics & $13(33 \%)$ & $5(25 \%)$ & 0.8 \\
\hline Fibrates & $3(8 \%)$ & $0(0 \%)$ & 0.5 \\
\hline Insulin & $1(3 \%)$ & $1(5 \%)$ & 0.6 \\
\hline Nitrates & $25(64 \%)$ & $13(65 \%)$ & 0.9 \\
\hline Oral antidiabetics & $2(5 \%)$ & $1(5 \%)$ & 0.9 \\
\hline Statins & $33(83 \%)$ & $16(80 \%)$ & 0.9 \\
\hline \multicolumn{4}{|l|}{ Laboratory tests * } \\
\hline Total cholesterol (mg/dl) & $193 \pm 37$ & $193 \pm 29$ & 1.0 \\
\hline LDL cholesterol (mg/dl) & $122 \pm 43$ & $112 \pm 35$ & 0.4 \\
\hline HDL cholesterol (mg/dl) & $46 \pm 14$ & $50 \pm 10$ & 0.3 \\
\hline Lipoprotein(a) (mg/L) & $24 \pm 24$ & $24 \pm 22$ & 0.5 \\
\hline Triglycerides (mg/dl) & $122 \pm 65$ & $150 \pm 73$ & 0.1 \\
\hline Apolipopotein A1 (mg/dl) & $147 \pm 20$ & $154 \pm 22$ & 0.2 \\
\hline Apoliprotein B (mg/dl) & $108 \pm 19$ & $100 \pm 22$ & 0.2 \\
\hline Apolipoprotein B/A1 (ratio) & $0.74 \pm 0.13$ & $0.66 \pm 0.21$ & 0.1 \\
\hline Fibrinogen (mg/dl) & $285 \pm 78$ & $299 \pm 85$ & 0.3 \\
\hline
\end{tabular}

${ }^{*}$ Data of laboratory tests are mean values of measurements obtained at time of initial and follow-up IVUS examinations. $A C E=$ angiotensin-converting enzyme; $H D L=$ high-density lipoprotein; $L D L=l o w$ density lipoprotein. 
Table 3. Baseline Intravascular Ultrasound Data

\begin{tabular}{|c|c|c|c|}
\hline & $\begin{array}{c}\text { Group A } \\
\text { (vessel area } \\
\text { changes }>0) \\
(n=40)\end{array}$ & $\begin{array}{c}\text { Group B } \\
\text { (vessel area } \\
\text { changes } \leq 0) \\
(n=20)\end{array}$ & $\mathbf{p}$ \\
\hline Vessel area $\left(\mathrm{mm}^{2}\right)$ & $24.1 \pm 5.4$ & $27.6 \pm 5.8$ & $<0.05$ \\
\hline Lumen area $\left(\mathrm{mm}^{2}\right)$ & $14.9 \pm 3.9$ & $17.3 \pm 4.6$ & $<0.05$ \\
\hline Plaque + media area $\left(\mathrm{mm}^{2}\right)$ & $9.3 \pm 3.7$ & $10.2 \pm 4.1$ & 0.4 \\
\hline Plaque burden (\%) & $38.1 \pm 10.5$ & $36.9 \pm 11.6$ & 0.7 \\
\hline Total arc of calcium $\left({ }^{\circ}\right)$ & $74 \pm 112$ & $71 \pm 81$ & 0.8 \\
\hline Plaque composition & & & 0.4 \\
\hline Soft & $10(25 \%)$ & $6(30 \%)$ & \\
\hline Fibrous & $11(28 \%)$ & $9(45 \%)$ & \\
\hline Mixed & $4(10 \%)$ & $1(5 \%)$ & \\
\hline Calcified & $15(38 \%)$ & $4(20 \%)$ & \\
\hline
\end{tabular}

\section{Serial IVUS data}

Because of the mild but significant difference in baseline IVUS data between groups A and B, percent serial annual changes were compared (Table 4). The 40 group $A$ plaques (those with positive remodeling behavior) showed an annual increase in vessel area of $2.1 \pm 2.1 \mathrm{~mm}^{2} /$ year, whereas group B plaques (those with negative or no serial arterial remodeling) had a reduction in vessel area of $-3.0 \pm 2.7 \mathrm{~mm}^{2} /$ year. Obviously, the increase versus decrease in vessel area was by definition of the 2 groups. Group A lesions had a mild annual increase in lumen area that occurred despite a mild increase in plaque+media area (Table 3 and Figure 1). Thus, overall in group A lesions, there was overcompensation for the increase in plaque+media area by a greater increase in vessel area. However, overcompensation was seen in only 19 lesions in group A patients $(32 \%$ of the total cohort). These 19 lesions had an annual plaque+media increase of $1.4 \pm 0.9 \mathrm{~mm} 2 /$ year and an annual vessel increase of $3.0 \pm 2.4 \mathrm{~mm}^{2} / y e a r$, resulting in an annual lumen increase of $1.6 \pm 2.1 \mathrm{~mm}^{2} /$ year. Ín group B lesions, the lumen reduction of $-2.8 \pm 2.6 \mathrm{~mm}^{2} /$ year was a result of only a decrease in vessel area; overall, plaque+media area showed almost no change (Figure 1). In fact, an 
increase in plaque+media cross-sectional area was found in no more than 9 group B patients $(45 \%)$, but in 32 group A patients $(80 \%)(p=0.02)$.

Table 4. Serial Intravascular Ultrasound Measurements (groups A and B)

\begin{tabular}{lccc}
\hline & $\begin{array}{c}\text { Group A } \\
(\text { vessel area } \\
\text { changes }>\mathbf{0}) \\
(\mathbf{n = 4 0 )}\end{array}$ & $\begin{array}{c}\text { Group B } \\
(\text { vessel area } \\
\text { changes } \leq \mathbf{0})\end{array}$ & $\mathbf{p}$ \\
$(\mathbf{n = 2 0 )}$ & $-3.0 \pm 2.7$ & $<0.0001$ \\
Changes in vessel area/yr $\left(\mathrm{mm}^{2}\right)$ & $2.1 \pm 2.1$ & $-10.1 \pm 7.6$ & $<0.0001$ \\
Changes in vessel area/yr (\%) & $9.4 \pm 9.3$ & $-2.8 \pm 2.6$ & $<0.0001$ \\
Changes in lumen area/yr $\left(\mathrm{mm}^{2}\right)$ & $0.9 \pm 2.3$ & $-15.0 \pm 11.8$ & $<0.0001$ \\
Changes in lumen area/yr $(\%)$ & $7.0 \pm 16.2$ & $-0.2 \pm 1.4$ & $<0.001$ \\
Changes in plaque + media area/yr $\left(\mathrm{mm}^{2}\right)$ & $1.2 \pm 1.3$ & $1.0 \pm 14.5$ & $<0.001$ \\
\hline Changes in plaque + media area/yr $(\%)$ & $16.6 \pm 18.2$ & & \\
\hline
\end{tabular}

Figure 1. Serial changes of the IVUS parameters. Group A plaques (changes in vessel area $>0 ; n=40$ ) showed, on average, a mild annual lumen crosssectional area increase (sign of overcompensation) as a result of vessel increase and despite plaque+media increase. Group $B$ lesions (changes in vessel area $\leq 0 ; n=20$ ) showed a considerable annual lumen loss, almost exclusively as a result of vessel area decrease (vessel shrinkage)

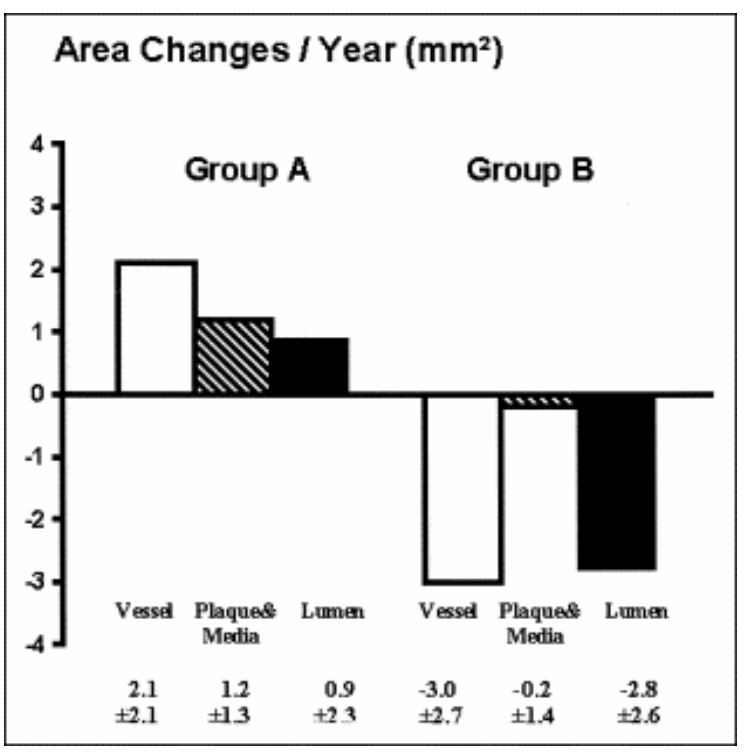

There was no change in IVUS plaque composition during follow-up. Similarly, groups $A$ and $B$ showed almost no change in total arc of calcium at follow-up up $\left(77 \pm 109^{\circ}\right.$ and $73 \pm 93^{\circ} ; p=0.7$ vs baseline $)$. 
Correlations among changes in vessel, lumen, and plaque + media area

Relations between percent annual changes in lumen, plaque+media, and vessel area are displayed in Figure 2. The percent annual changes in lumen area correlated strongly with the percent annual changes in vessel area $(r=0.84)$, but not with the plaque+media area $(p=0.8)$. In addition, there was a modest correlation between percent annual changes in vessel area versus percent annual changes in plaque+media area $(r=0.46)$.

Figure 2. Relations between annual changes of vessel, plaque+media (Plaque\&Media Area), and lumen area. Lumen cross-sectional area changes showed a strong linear relation to vessel area changes $(A)$, but not with plaque+media area changes $(B)$ (therefore no line is shown in $B$ ). Moreover, there was a relation between percent annual changes in vessel versus plaque+media area (C). $A$, closed dots versus open dots represent plaque+media increase versus decrease; $B$, vessel decrease versus increase; $C$, lumen decrease versus increase, respectively.

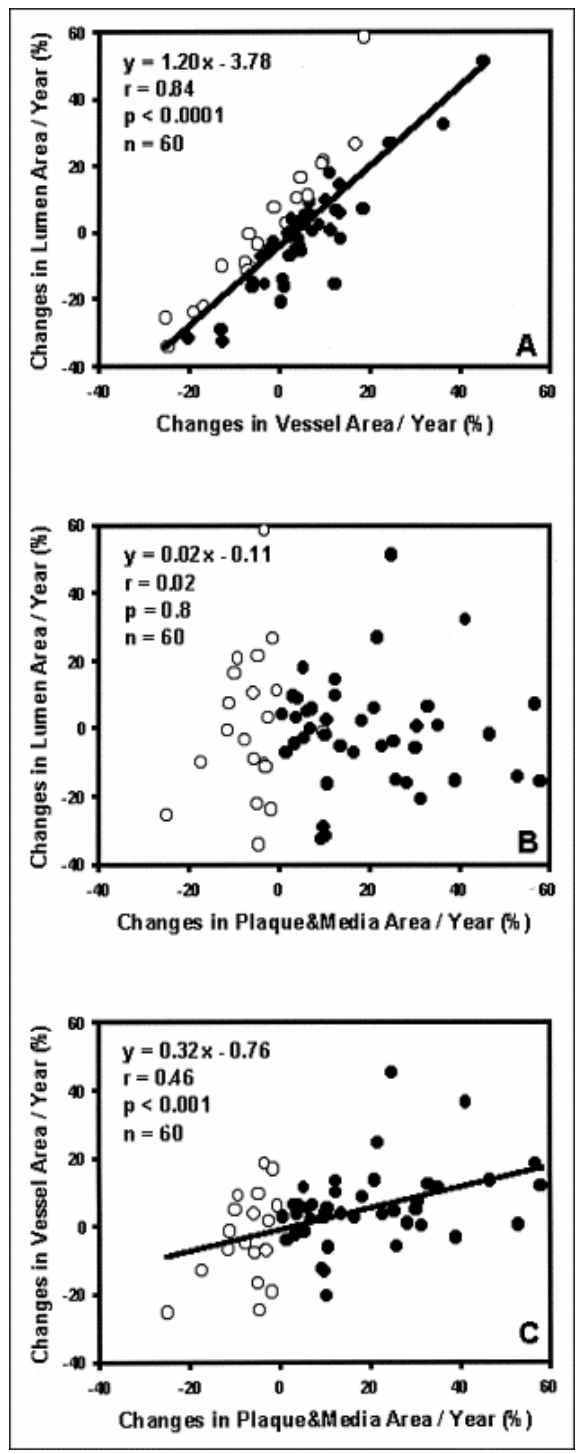


Comparison of lesions with versus without lumen decrease

Plaques with lumen decrease $(n=32)$ were associated with an annual decrease in vessel area of $-1.5 \pm 2.9 \mathrm{~mm}^{2} /$ year, whereas plaques with lumen increase $(n=28)$ were associated with an annual increase in vessel area of $2.6 \pm 2.3 \mathrm{~mm}^{2} /$ year $(p<0.0001$; Table 5). There was no difference in the (mild) annual plaque+media area increase between the 2 groups $(p=0.9)$.

Table 5. Serial Intravascular Ultrasound Measurements (lesions with vs. without lumen decrease)

\begin{tabular}{|c|c|c|c|}
\hline & $\begin{array}{c}\text { Lumen area } \\
\text { Changes } \\
>0 \\
(n=28)\end{array}$ & $\begin{array}{c}\text { Lumen area } \\
\text { Changes } \\
\leq 0 \\
(n=32)\end{array}$ & $\mathbf{p}$ \\
\hline Changes in vessel area/yr $\left(\mathrm{mm}^{2}\right)$ & $2.6 \pm 2.3$ & $-1.5 \pm 2.9$ & $<0.0001$ \\
\hline Changes in vessel area/yr (\%) & $11.4 \pm 10.2$ & $-4.6 \pm 9.7$ & $<0.0001$ \\
\hline Changes in lumen area/yr $\left(\mathrm{mm}^{2}\right)$ & $1.9 \pm 2.0$ & $-2.3 \pm 2.2$ & $<0.0001$ \\
\hline Changes in lumen area/yr (\%) & $13.8 \pm 14.3$ & $-12.7 \pm 10.2$ & $<0.0001$ \\
\hline Changes in plaque + media area/yr $\left(\mathrm{mm}^{2}\right)$ & $0.7 \pm 1.2$ & $0.8 \pm 1.7$ & 0.9 \\
\hline Changes in plaque + media area/yr $(\%)$ & $10.7 \pm 17.0$ & $12.1 \pm 19.9$ & 0.8 \\
\hline
\end{tabular}

Comparison of lesions with versus without plaque + media increase

Lesions with plaque+media increase $(n=41)$ were associated with an annual increase in vessel area of $1.2 \pm 2.9 \mathrm{~mm}^{2} /$ year, whereas lesions with plaque+media decrease $(n=19)$ were associated with an annual decrease in vessel area of $-1.2 \pm 3.8 \mathrm{~mm}^{2} /$ year $(p<0.02$; Table 6$)$. There was no difference in the annual lumen area decrease between the 2 groups $(p=0.9)$. 
Table 6. Serial Intravascular Ultrasound Measurements (lesions with vs without plaque increase)

\begin{tabular}{lccc}
\hline & $\begin{array}{c}\text { Plaque + media } \\
\text { Area Changes } \\
\mathbf{0}\end{array}$ & $\begin{array}{c}\text { Plaque + media } \\
\text { Area Changes } \\
\mathbf{( n = \mathbf { 0 }}\end{array}$ & $\mathbf{p}$ \\
$\mathbf{( n = 1 9 )}$ & $\mathbf{p}$ & \\
Changes in vessel area/yr $\left(\mathrm{mm}^{2}\right)$ & $1.2 \pm 2.9$ & $-1.2 \pm 3.8$ & $<0.02$ \\
Changes in vessel area/yr $(\%)$ & $5.8 \pm 11.7$ & $-3.3 \pm 12.9$ & $<0.01$ \\
Changes in lumen area/yr $\left(\mathrm{mm}^{2}\right)$ & $-0.3 \pm 2.8$ & $-0.4 \pm 3.4$. & 0.9 \\
Changes in lumen area/yr $(\%)$ & $-1.1 \pm 16.1$ & $-1.2 \pm 22.2$ & 0.6 \\
Changes in plaque + media area/yr $\left(\mathrm{mm}^{2}\right)$ & $1.5 \pm 1.0$ & $-0.8 \pm 1.0$ & $<0.0001$ \\
Changes in plaque + media area/yr $(\%)$ & $20.0 \pm 15.8$ & $-7.1 \pm 6.1$ & $<0.0001$ \\
\hline
\end{tabular}

\section{Discussion}

In the present study, we analyzed serial IVUS studies of 60 left main coronary arteries obtained $18.4 \pm 9.4$ months apart to assess the arterial remodeling behavior (i.e., changes in vessel dimensions) in nonstenotic atherosclerotic lesions. Serial long-term reduction in lumen size resulted from vessel shrinkage (sometimes despite plaque+media decrease), plaque+media increase (with or without vessel increase), or both. Overall, the percent annual changes in lumen size showed a strong relation to the changes in only vessel area $(r=0.84)$, but not to changes in plaque+media size. In all, 40 lesions (67\%) had positive arterial remodeling behavior, and $20(33 \%)$ negative remodeling behavior. The groups differed neither in baseline plaque burden nor in demographics, laboratory data, or medications. Positive remodeling lesions more often showed plaque+media increase than negative remodeling lesions and had, on average, a mild annual lumen increase; this indicated overcompensation of vessel increase for plaque+media increase. Conversely, negative remodeling lesions had significant lumen reduction as a result of only vessel shrinkage.

\section{Serial IVUS assessment of coronary artery dimensions and remodeling}

Our findings are in accord with those of Shiran et al. ${ }^{22}$ who previously studied 31 left main coronary arteries and demonstrated that lumen changes during 6-month follow-up resulted primarily from positive versus negative arterial remodeling. 
Schartl et al. ${ }^{23}$ studied 65 patients treated with atorvastatin and 66 patients treated with usual care and found an increase in IVUS vessel volume in both groups during 12 month follow-up (i.e., compensatory remodeling); although no statistically significant difference was observed, this increase was more pronounced in the usual-care group. Thus far, only 1 group - Takagi et al. ${ }^{21}$ - has reported a longer IVUS follow-up (36 months) than our present observational study. Thirteen patients treated with pravastatin were compared with 12 controls. There was an increase in vessel size only in controls. ${ }^{21}$ Serial IVUS studies, like the current analysis, are the only absolute way to assess remodeling behavior independent of changes in reference segment plaque burden, vessel tapering, and so forth. ${ }^{20,26}$

\section{Serial IVUS assessment of coronary plaque composition}

Schartl et al. ${ }^{23}$ used a sophisticated computer-aided gray-scale analysis in their pharmacologic intervention study to demonstrate an increase in plaque hyperechogenicity (likely due to a change in plaque composition, i.e., an increase in fibrous tissue). However, our present study showed no change in IVUS plaque composition when assessed with conventional visual IVUS analysis. ${ }^{20}$ Visual analysis may be limited in its ability to assess changes in plaque composition. Recent developments in radiofrequency backscatter analysis show promise in quantifying patient plaque components. ${ }^{27}$

\section{Study limitations}

Although, by most standards, this was a large serial IVUS study, we evaluated only 60 patients; all studies with long-term ( $\geq 12$ months) serial assessment of remodeling are limited to a relatively small number of patients. This study was performed in left main coronary arteries and did not include ostial or heavily calcified lesions; therefore, our findings may not be applicable to all coronary segments or to ostial or heavily calcified lesions of the left main stem. The reason for excluding heavily calcified lesions in this study (as in all IVUS studies of atherosclerosis that assess remodeling and progression/regression) was that ultrasound cannot penetrate calcified tissue to measure the total vessel area and assess remodeling. Data in this study are unique and may well reflect clinical reality. However, because retrospective analyses of prospectively acquired data (demographics, medication, and laboratory tests) were performed, we cannot rule out a certain selection bias. We were able to include only patients with significant coronary artery disease who were admitted for repeat cardiac catheterization $\geq 12$ months after baseline (this limitation applies to both groups); therefore, the findings of the present study may not be applicable to the general population. All patients 
were Caucasian and most were men. We used 2 IVUS systems in the present study. The pull-back device of the mechanical IVUS system is known to be exceptionally reliable (which may be helpful to match target sites), whereas the electronic system is not affected by nonuniform rotation distortion; however, when we compared the data from the 2 different IVUS systems, we found no differences. Also, separate linear regression analyses in data sets that were obtained by one or the other IVUS system provided almost identical results. Furthermore, patients underwent imaging with the same system at index and at follow-up.

Thus, our serial IVUS data show that lumen reduction may result from vessel shrinkage (sometimes despite plaque+media decrease), plaque+media increase (with or without increase in vessel dimensions), or both vessel shrinkage and plaque+media increase. Overall, long-term changes in lumen size showed a strong relation to the remodeling behavior, but not to changes in plaque size. More than $30 \%$ of atherosclerotic left main lesion showed negative remodeling behavior, which contributed significantly to the overall lumen loss (even in the absence of plaque+media increase), and showed no relation to patient characteristics or initial plaque burden

\section{References}

1. Glagov S, Weisenberg E, Zarins CK, et al. Compensatory enlargement of human atherosclerotic coronary arteries. N Engl J Med 1987;316:1371-1375.

2. Clarkson TB, Prichard RW, Morgan TM, et al. Remodeling of coronary arteries in human and nonhuman primates. JAMA 1994;271:289-294.

3. Pasterkamp G, Wensing PJW, Post MJ, et al. Paradoxical arterial wall shrinkage may contribute to luminal narrowing of human atherosclerotic femoral arteries. Circulation 1995;91:1444-1449.

4. Nishioka T, Luo H, Eigler NL, et al. Contribution of inadequate compensatory enlargement to development of human coronary artery stenosis: an in vivo intravascular ultrasound study. J Am Coll Cardiol 1996;27:1571-1576.

5. Mintz GS, Kent KM, Pichard AD, et al. Contribution of inadequate arterial remodeling to the development of focal coronary artery stenoses: an intravascular ultrasound study. Circulation 1997;95:1791-1798.

6. von Birgelen C, Airiian SG, Mintz GS, et al. Variations of remodeling in response to left main atherosclerosis assessed with intravascular ultrasound in vivo. Am J Cardiol 1997;80:1408-1413.

7. von Birgelen C, Mintz GS, de Very EA, et al. Atherosclerotic coronary lesions with inadequate compensatory enlargement have smaller plaque and vessel volumes: observations with threedimensional intravascular ultrasound in vivo. Heart 1998;79:137-143.

8. von Birgelen C, Mintz GS, Sieling C, et al. Relation between plaque composition and vascular remodeling in coronary lesions with different degrees of lumen narrowing as assessed with threedimensional intravascular ultrasound in patients with stable angina pectoris. Am J Cardiol 2003;91: 1103-1107.

9. Ge J, Erbel R, Zamorano J, et al. Coronary arterial remodeling in atherosclerotic disease: an intravascular ultrasound study in vivo. Coron Artery Dis 1993;4:981-986. 
10. Tauth J, Pinnow E, Sullebarger JT, et al. Predictors of coronary arterial remodeling patterns in patients with myocardial ischemia. Am J Cardiol 1996;80: 1352-1355.

11. Sabate M, Kay IP, de Feyter PJ, et al. Remodeling of atherosclerotic coronary arteries varies in relation to location and composition of plaque. Am J Cardiol 1999,84:135-140.

12. Weissman NJ, Sheris SJ, Chari R, et al. Intravascular ultrasonic analysis of plaque characteristics associated with coronary artery remodeling. Am J Cardiol 1999;84:37-40.

13. FuessI RT, Kranenberg E, Kiausch $U$, et al. Vascular remodeling in atherosclerotic coronary arteries is affected by plaque composition. Coron Artery Dis 2001;12:91-97.

14. Gyöngyösi M, Yang P, Hassan A, et al. Arterial remodelling of native human coronary arteries in patients with unstable angina pectoris: a prospective intravascular ultrasound study. Heart 1999;82:68-74.

15. Schoenhagen P, Ziada KM, Kapadia SR, et al. Extent and direction of arterial remodeling in stable versus unstable coronary syndromes: an intravascular ultrasound study. Circulation 2000;101:598603.

16. von Birgelen C, Klinkhart W, Mintz GS, et al. Plaque distribution and vascular remodeling of ruptured and nonruptured coronary plaques in the same vessel: an intravascular ultrasound study in vivo. J Am Coll Cardiol 2001;371864-1870.

17. Nakamura M, Nishikawa $\mathrm{H}$, Mukai $\mathrm{S}$, et al. Impact of coronary artery remodeling on clinical presentation of coronary artery disease: an intravascular ultrasound study. J Am Coll Cardiol 2001;37:63-69.

18. Ward MR, Jeremias A, Huegel $H$, et al. Accentuated remodeling on the upstream side of atherosclerotic lesions. Am J Cardiol 2000;85:523-526.

19. Hamasaki S, Higano ST, Suwaidi JA, et al. Cholesterol-lowering treatment is associated with improvement in coronary vascular remodeling and endothelial function in patients with normal or mildly diseased coronary arteries. Arterioscler Thromb Vasc Biol 2000;20:737-741.

20. Mintz GS, Nissen SE, Anderson WD, et al. American College of Cardiology clinical expert consensus document on standards for acquisition, measurement and reporting of intravascular ultrasound studies (IVUS). J Am Coll Cardiol 2001;37:1478-1492.

21. Takagi T, Yoshida K, Akasaka T, et al. Intravascular ultrasound analysis of reduction in progression of coronary narrowing by treatment with pravastatin. Am J Cardiol 1997;79:1673-1676.

22. Shiran A, Mintz GS, Leiboff B, et al. Serial volumetric intravascular ultrasound assessment of arterial remodeling in left main coronary artery disease. Am J Cardiol 1999;83:1427-1432.

23. Schartl M, Bocksch W, Koschyk DH, et al. Use of intravascular ultrasound to compare effects of different strategies of lipid-lowering therapy on plaque volume and composition in patients with coronary artery disease. Circulation 2001;104: 387-392.

24. von Birgelen C, Hartmann M, Mintz GS, et al. Relation between progression and regression of atherosclerotic left main coronary artery disease and serum cholesterol levels as assessed with serial long-term ( $\geq 12$ months) follow-up intravascular ultrasound. Circulation 2003;108:2757-2762.

25. Baumgart $\mathrm{D}$, Haude $\mathrm{M}, \mathrm{Ge} \mathrm{J}$, et al. Online intergration of intravascular ultrasound images into angiographic images. Cathet Cardiovasc Diagn 1996;39:328-329.

26. Schoenhagen P, Zidada KM, Vince DG, et al. Arterial remodeling and coronary artery disease: the concept of "dilated" versus "obstructive" coronary atherosclerosis. J Am Coll Cardiol 2001;38:297306.

27. Nair A, Kuban BD, Tuzcu EM, et al. Coronary plaque classification with intravascular ultrasound radiofrequency data analysis. Circulation 2002;106: 2200-220. 



\section{Chapter 7}

\section{Remodeling Index Compared to Actual Vascular Remodeling in Atherosclerotic Left Main Coronary Arteries as Assessed With Long-Term ( $\geq 12$ Months) Serial Intravascular Ultrasound}

Clemens von Birgelen, Marc Hartmann, Gary S. Mintz, Dirk Böse, Holger Eggebrecht, Till Neumann, Mario Gössl, Heinrich Wieneke, Axel Schmermund, Martin G. Stoel, Patrick M.J. Verhorst, Raimund Erbel

Reprinted with permission from:

Journal of the American College of Cardiology 2006;47(7):1363-1368.

(C) 2006 American College of Cardiology Foundation / Elsevier Inc. 



\section{Abstract}

Objectives: We present the remodeling index (RI) versus serial intravascular ultrasound (IVUS) data.

Background: The RI, derived by comparing lesion external elastic membrane (EEM) crosssectional area versus the reference at one time point, is used in various IVUS studies as a substitute of true remodeling (change in EEM over time), assuming that it represents true remodeling.

Methods: We studied 46 non-stenotic left main arteries using serial IVUS (follow-up $18 \pm 8$ months). Plaques were divided into subgroups according to the follow-up RI: follow-up RI>1 ( $n=$ 27 ) versus follow-up RI $\leq 1(n=19)$.

Results: Lesions with a follow-up $\mathrm{Rl}>1$ had an increase in lumen despite an increase in plaque because of an increase in EEM. Conversely, lesions with a follow-up RI $\leq 1$ had a reduction in lumen as a result of both a plaque increase and EEM decrease. Overall, the follow-up RI correlated directly with changes in lesion site EEM (baseline-to-follow-up). Although there was no correlation between the follow-up RI and changes in reference EEM area, changes in reference EEM area did correlate directly with changes in lesion EEM area. In nearly $90 \%$ of lesions with a follow-up $\mathrm{RI}>1$, there was a previously documented increase in EEM area. Using multivariate linear regression analysis, the follow-up RI was dependent on the baseline RI, the increase in lesion EEM area, and the decrease in reference EEM area. The follow-up RI was not dependent on changes in lesion plaque area.

Conclusions: The vast majority of left main lesions with a remodeling index $>1$ had evidence of a previous increase in lesion-site EEM area.

\section{Introduction}

Both histopathologic ${ }^{1-3}$ and intravascular ultrasound (IVUS) analyses ${ }^{3-12}$ have described positive and negative remodeling of atherosclerotic lesions, typically by comparing absolute lesion-site external elastic membrane (EEM) measurements to the reference segments at a single point in time. For example, a remodeling index (RI) can be derived as lesion-site EEM cross-sectional area (CSA) divided by a pre-defined reference vessel area; $\mathrm{Rl}>1$ is considered evidence for positive remodeling, whereas $\mathrm{RI} \leq 1$ suggests intermediate or negative remodeling. However, for many reasons (such as single time-point observation as well as reference segment selection, tapering, plaque burden, and remodeling), these indexes are acknowledged to be only indirect evidence of remodeling. ${ }^{13,14}$ Direct evidence of remodeling requires serial EEM measurements obtained at two (or more) time points. $^{15-19}$

In the present serial IVUS study in non-stenotic left main coronary lesions, we assessed whether the more commonly used indirect assessment of remodeling (i.e., the $\mathrm{RI}$ ) reflects true vascular remodeling (i.e., changes in lesion-site EEM 
CSA). Therefore, we compared the RI at follow-up with the preceding changes in vessel dimensions.

\section{Methods}

\section{Study population}

We analyzed serial IVUS data of 46 patients who had hemodynamically nonsignificant de novo left main atherosclerotic lesions and reliable reference segments; these patients were from a population of 60 lesions that has previously been reported. ${ }^{18,19}$ Fourteen left main lesions were excluded because there was a lack of a well-defined reference. ${ }^{14}$ All patients met the following criteria: 1) serial high-quality IVUS imaging of the entire left main stem $\geq 12$ months apart; 2) calcifications that did not limit the quantitative assessment of vessel area (shadowing $\leq 75^{\circ}$ of the adventitial circumference by individual calcific deposits); 3 ) non-ostial target site location; 4) angiographic lumen diameter stenosis $<30 \%$ ("worst view" visual assessment); 5) no intervention in the very proximal left anterior descending or circumflex coronary artery segments because these interventions could have affected the left main artery; and 6) well-defined lesion and reference image slices. Patients were examined in the Essen University Cardiac Catheterization Laboratory with a follow-up of $17.7 \pm 8.1$ months (median 15 months, range 12 to 49 months). As previously reported, this represents a consecutive series of patients who underwent initial IVUS examination during coronary intervention and then returned after $\geq 1$ year for repeat intervention during which another IVUS study was performed. ${ }^{18}$ The IVUS study was approved by the local council on human research. All patients signed a written informed consent form as approved by the local medical ethics committee.

\section{Demographics, medications, and lipid status}

Demographics including cardiovascular risk factors, medications, and the lipid status were prospectively recorded in our laboratory including diabetes mellitus and hypertension (both medication-dependent only); hypercholesterolemia (medicationdependent, total serum cholesterol $>\mathbf{2 0 0} \mathrm{mg} / \mathrm{dl}$, or low-density lipoprotein cholesterol $>160 \mathrm{mg} / \mathrm{dl}$ ); history of smoking; and family history of coronary artery disease. Data of laboratory tests were means of the baseline and follow-up values. Medications were recorded only if drugs were taken for $>50 \%$ of the follow-up interval (e.g., clopidogrel for four weeks was not tabulated). Plasma concentrations 
of total cholesterol, low-density lipoprotein cholesterol, high-density lipoprotein cholesterol, and triglycerides were measured by standard enzymatic methods.

\section{IVUS imaging}

Intravascular ultrasound was performed as previously described. ${ }^{18}$ In brief, IVUS studies were performed during percutaneous coronary interventions of mid- or distal left anterior or left circumflex arteries after intracoronary injections of $200 \mu \mathrm{g}$ nitroglycerin. Two commercial systems were used: a mechanical sector scanner (Boston Scientific Corporation, San Jose, California) incorporating a $30-\mathrm{MHz}$ single-element beveled transducer or a solid-state device (Endosonics, Rancho Cordova, California). Importantly, at Essen University, if a patient undergoes imaging with one IVUS system during an index procedure, the same IVUS system is used at follow-up. Slow continuous pullbacks of the IVUS transducer were started as distal as possible in one of the left coronary arteries and were generally performed using a motorized pullback device (at $0.5 \mathrm{~mm} / \mathrm{s}$ ); IVUS images of the entire pullback were recorded on 0.5 -inch high-resolution s-VHS tape for off-line analysis. In addition, a dedicated image-in-image system (Echo-Map, Siemens, Erlangen, Germany $)^{20}$ was used to record the "angiographic" position of the IVUS probe together with the corresponding IVUS image - especially at sites of characteristic landmarks (i.e., calcifications or unusual plaque shapes) and/or the target site. Follow-up IVUS studies were performed (using the same IVUS system as initially) during repeat coronary interventions or during IVUS examinations of ambiguous left coronary lesions or (clinically driven) follow-up catheterizations.

\section{Quantitative IVUS analysis}

The target lesion site image slice was the slice with the smallest lumen CSA. ${ }^{18}$ If there were several slices with equal lumen size, the one with the largest EEM and plaque and media (P\&M = EEM - lumen) CSA was analyzed. ${ }^{6,14} \mathrm{~A}$ left main stem reference image slice was selected as the most normal-looking cross section (largest lumen with smallest P\&M) distal or proximal to the target lesion. Exact matching of the initial and follow-up IVUS studies was ensured using side-by-side comparison of the serial IVUS video sequences along with information of the pullback speed ${ }^{18}$; the operators' recorded comments (on videotape); and characteristic calcifications, vascular and perivascular landmarks, and plaque shapes. If required, the X-ray sequences of the dedicated image-in-image system (Echo-Map) were revisited to optimize matching. ${ }^{18,20}$

The lumen CSA was measured by tracing the leading edge of the intima. The EEM CSA was measured by tracing the leading edge of the adventitia. As in many 
previous IVUS studies, P\&M was used as a measure of atherosclerotic plaque because IVUS cannot measure media thickness accurately. Lumen and plaque eccentricity ([maximum lumen/plaque diameter minus minimum lumen/plaque diameter] divided by maximum lumen/plaque diameter) was calculated. In our laboratory, the intraclass correlation coefficient is 0.99 for repeated measurements of EEM, 0.96 for lumen, and 0.99 for P\&M CSA.

We calculated the changes between $(\Delta)$ the initial and follow-up IVUS data. To compensate for the variation in follow-up interval and to obtain comparable data, measurements were normalized for the length of the follow-up period and annual changes (i.e., changes per year) were reported and compared.

Direct (serial IVUS) measurement of arterial remodeling and RI

The change in lesion-site EEM CSA was the direct measure of arterial remodeling (i.e., serial arterial remodeling or "remodeling behavior"). The RI was calculated at follow-up as lesion site divided by reference EEM CSA ${ }^{13,14}$ and used to classify lesions into positive remodeling $(R \mid>1)$ and intermediate/negative remodeling $(\mathrm{RI} \leq 1)$.

\section{Statistics}

Analyses were performed with SPSS 10.0.7 (Microsoft, Redmond, Washington) and MedCalc (version 4.16, Mariakerke, Belgium) software packages for Windows. Dichotomous data are presented as frequencies and compared using chi-square statistics; Fisher exact test was applied if at least one expected frequency was $\leq 5$. Quantitative data are presented as mean values $\pm 1 \mathrm{SD}$ and compared using Student $t$ test and linear regression analysis. A value of $p<0.05$ was considered significant.

\section{Results}

\section{Study population}

Plaques were divided into two groups according to the follow-up RI: follow-up RI>1 $(n=27)$ versus $R I \leq 1 \quad(n=19)$. The actual $R I$ values were $1.15 \pm 0.16$ and $0.89 \pm 0.09$, respectively $(p<0.0001)$. There was no difference in demographics, medication, and lipid status between the two groups (Table 1). 
Table 1 (part1). Patient Demographics, Medication, and Lipid Status

\begin{tabular}{|c|c|c|c|}
\hline & $\begin{array}{c}\text { Follow-Up RI > } 1 \\
(n=27)\end{array}$ & $\begin{array}{c}\text { Follow-Up RI } \leq 1 \\
(n=19)\end{array}$ & p Value \\
\hline Time of follow-up (months) & $17.9 \pm 7.1$ & $17.4 \pm 9.6$ & 0.8 \\
\hline Age (yrs) & $59 \pm 9$ & $57 \pm 9$ & 0.5 \\
\hline Men & $24(89 \%)$ & $17(89 \%)$ & $1.0^{*}$ \\
\hline Body mass index $\left(\mathrm{kg} / \mathrm{m}^{2}\right)$ & $27 \pm 3$ & $26 \pm 3$ & 0.3 \\
\hline Hypercholesterolemia & $25(93 \%)$ & $16(84 \%)$ & $0.6^{*}$ \\
\hline Systemic arterial hypertension & $21(78 \%)$ & $13(68 \%)$ & $0.5^{*}$ \\
\hline Diabetes mellitus & $5(19 \%)$ & $1(5 \%)$ & $0.4^{*}$ \\
\hline Smoker & $10(37 \%)$ & $5(26 \%)$ & $0.7 \dagger$ \\
\hline Family history of coronary disease & $6(22 \%)$ & $4(21 \%)$ & $1.0^{*}$ \\
\hline Previous myocardial infarction & $10(37 \%)$ & $4(21 \%)$ & $0.3^{*}$ \\
\hline 1 vessel narrowed $>50 \%$ & $10(37 \%)$ & $10(53 \%)$ & $0.5 \dagger$ \\
\hline 2 vessels narrowed $>50 \%$ & $9(33 \%)$ & $4(21 \%)$ & $0.5^{*}$ \\
\hline 3 vessels narrowed $>50 \%$ & $8(30 \%)$ & $5(26 \%)$ & $0.9 \dagger$ \\
\hline Stable angina CCS class I & $8(30 \%)$ & $7(37 \%)$ & $0.8 \dagger$ \\
\hline Stable angina CCS class II & $10(37 \%)$ & $8(42 \%)$ & $1.0 \dagger$ \\
\hline Stable angina CCS class III & $6(22 \%)$ & $3(16 \%)$ & $0.7^{*}$ \\
\hline Unstable angina & $3(11 \%)$ & $1(5 \%)$ & $0.6^{*}$ \\
\hline
\end{tabular}

* Compared using Fisher exact test

${ }^{\dagger}$ compared using chi-square statistics

$R I=$ remodeling index . 
Table 1 (part2). Patient Demographics, Medication, and Lipid Status

\begin{tabular}{|c|c|c|c|}
\hline & $\begin{array}{c}\text { Follow-Up RI }>1 \\
(n=27)\end{array}$ & $\begin{array}{c}\text { Follow-Up RI } \leq 1 \\
(n=19)\end{array}$ & p Value \\
\hline \multicolumn{4}{|l|}{ Medication } \\
\hline Acetylsalicylic acid & $27(100 \%)$ & $19(100 \%)$ & $1.0^{*}$ \\
\hline ACE inhibitors & $9(33 \%)$ & $9(47 \%)$ & $0.5 \dagger$ \\
\hline Angiotensin receptor antagonists & $1(4 \%)$ & $0(0 \%)$ & $1.0^{*}$ \\
\hline Beta-blockers & $15(56 \%)$ & $9(47 \%)$ & $0.8 \dagger$ \\
\hline Calcium-channel blockers & $11(41 \%)$ & $4(21 \%)$ & $0.2^{*}$ \\
\hline Diuretics & $10(37 \%)$ & $3(16 \%)$ & $0.2^{*}$ \\
\hline Fibrates & $2(7 \%)$ & $1(5 \%)$ & $1.0^{*}$ \\
\hline Insulin & $1(4 \%)$ & $0(0 \%)$ & $1.0^{*}$ \\
\hline Nitrates & $19(70 \%)$ & $10(53 \%)$ & $0.4 \dagger$ \\
\hline Oral antidiabetics & $2(7 \%)$ & $1(5 \%)$ & $1.0^{*}$ \\
\hline Statins & $22(81 \%)$ & $14(74 \%)$ & $0.7^{*}$ \\
\hline \multicolumn{4}{|l|}{ Lipid status $\ddagger$} \\
\hline Total cholesterol (mg/dl) & $196 \pm 37$ & $189 \pm 37$ & 0.5 \\
\hline LDL cholesterol (mg/dl) & $123 \pm 46$ & $115 \pm 43$ & 0.6 \\
\hline HDL cholesterol (mg/dl) & $48 \pm 16$ & $47 \pm 10$ & 0.8 \\
\hline Triglycerides (mg/dl) & $128 \pm 71$ & $123 \pm 65$ & 0.8 \\
\hline \multicolumn{4}{|c|}{$\begin{array}{l}{ }^{*} \text { Compared using Fisher exact test } \\
{ }^{+} \text {compared using chi-square statistics } \\
{ }^{\ddagger} \text { data of laboratory tests are mean values of measurements obtained at time of initial and follow-up } \\
\text { intravascular ultrasound examinations. } \\
\text { ACE = angiotensin-converting enzyme; } H D L=\text { high-density lipoprotein; } L D L=\text { low-density lipoprotein; } \\
R I=\text { remodeling index. }\end{array}$} \\
\hline
\end{tabular}

\section{Follow-up IVUS remodeling}

In lesions with a follow-up RI>1, baseline reference EEM and lumen CSA were smaller and baseline RI was larger compared to lesions with a follow-up RI 1 (Table 2). The other baseline IVUS measurements - reference P\&M CSA and lesion EEM, lumen, and P\&M CSA as well as lesion plaque burden - were similar between the two groups. 
Table 2. Comparison of the Baseline IVUS Data According to Follow-Up Remodeling Index

\begin{tabular}{|c|c|c|c|}
\hline & $\begin{array}{c}\text { Follow-Up RI > } 1 \\
(n=27)\end{array}$ & $\begin{array}{l}\text { Follow-Up RI } \leq 1 \\
\qquad(n=19)\end{array}$ & p Value \\
\hline \multicolumn{4}{|l|}{ Baseline reference } \\
\hline EEM CSA $\left(\mathrm{mm}^{2}\right)$ & $22.4 \pm 5.8$ & $27.4 \pm 8.0$ & $<0.02$ \\
\hline Lumen CSA $\left(\mathrm{mm}^{2}\right)$ & $16.8 \pm 3.4$ & $21.7 \pm 6.9$ & $<0.01$ \\
\hline $\mathrm{P} \& \mathrm{M}$ CSA $\left(\mathrm{mm}^{2}\right)$ & $5.7 \pm 4.1$ & $5.7 \pm 2.2$ & 0.9 \\
\hline Plaque burden (\%) & $23.0 \pm 11.3$ & $21.1 \pm 7.1$ & 0.5 \\
\hline \multicolumn{4}{|l|}{ Baseline lesion } \\
\hline EEM CSA $\left(\mathrm{mm}^{2}\right)$ & $24.3 \pm 5.1^{*}$ & $25.7 \pm 6.2 \dagger$ & 0.4 \\
\hline Lumen CSA $\left(\mathrm{mm}^{2}\right)$ & $15.1 \pm 3.1^{*}$ & $16.1 \pm 4.9 \ddagger$ & 0.4 \\
\hline $\mathrm{P} \& \mathrm{M}$ CSA $\left(\mathrm{mm}^{2}\right)$ & $9.3 \pm 4.0 \ddagger$ & $9.6 \pm 3.1 \mp$ & 0.8 \\
\hline Plaque burden (\%) & $37.2 \pm 10.9 \ddagger$ & $37.8 \pm 9.3 \ddagger$ & 0.8 \\
\hline Lumen eccentricity & $0.18 \pm 0.10$ & $0.18 \pm 0.11$ & 1.0 \\
\hline Plaque eccentricity & $0.76 \pm 0.11$ & $0.79 \pm 0.09$ & 0.3 \\
\hline Baseline RI & $1.11 \pm 0.17$ & $0.96 \pm 0.11$ & $<0.001$ \\
\hline \multicolumn{4}{|c|}{$\begin{array}{l}\text { CSA = cross-sectional area; EEM = external elastic membrane; IVUS = intravascular ultrasound; } P C \\
=\text { plaque and media; } R I=\text { remodeling index. } \\
\text { Reference vs. lesion: } \\
{ }^{*} p<0.01 \\
\dagger p<0.05 \\
\neq p<0.001\end{array}$} \\
\hline
\end{tabular}

We then analyzed the baseline-to-follow-up changes in IVUS measurements in patients with follow-up left main stem $\mathrm{RI}>1$ versus follow-up $\mathrm{RI} \leq 1$ (Table 3 ). Changes in reference segment EEM, lumen, and P\&M CSA measurements were similar between the two groups. The two groups had similar increases in lesion P\&M CSA from baseline to follow-up. However, lesions with a follow-up RI>1 had an increase in minimum lumen CSA despite this increase in P\&M CSA; this was the result of an increase in lesion EEM CSA. Conversely, on average, lesions with a follow-up $\mathrm{RI} \leq 1$ had a reduction in minimum lumen CSA as a result of both, P\&M CSA increase and EEM CSA decrease. 
Table 3. Comparison of the Serial IVUS Data According to Follow-Up Remodeling Index

\begin{tabular}{|c|c|c|c|}
\hline & $\begin{array}{c}\text { Follow-Up RI }>1 \\
(n=27)\end{array}$ & $\begin{array}{c}\text { Follow-Up RI } \leq 1 \\
(n=19)\end{array}$ & p Value \\
\hline \multicolumn{4}{|l|}{ Reference changes } \\
\hline$\Delta \mathrm{EEM} \mathrm{CSA} / \mathrm{yr}\left(\mathrm{mm}^{2}\right)$ & $0.9 \pm 3.0$ & $1.0 \pm 5.0$ & 0.9 \\
\hline$\triangle \mathrm{EEM} \mathrm{CSA/yr} \mathrm{( \% )}$ & $5.8 \pm 16.2$ & $7.1 \pm 21.8$ & 0.8 \\
\hline$\Delta$ Lumen CSA/yr $\left(\mathrm{mm}^{2}\right)$ & $0.8 \pm 2.4$ & $1.1 \pm 3.5$ & 0.8 \\
\hline$\Delta$ Lumen CSA/yr (\%) & $6.2 \pm 15.4$ & $8.5 \pm 20.2$ & 0.7 \\
\hline$\triangle \mathrm{P} \& \mathrm{M}$ CSA/yr $\left(\mathrm{mm}^{2}\right)$ & $0.1 \pm 1.6^{*}$ & $-0.1 \pm 2.2$ & 0.8 \\
\hline$\triangle \mathrm{P} \& \mathrm{M}$ CSA/yr (\%) & $8.6 \pm 29.5$ & $9.8 \pm 48.0$ & 0.9 \\
\hline \multicolumn{4}{|l|}{ Lesion changes } \\
\hline$\triangle \mathrm{EEM} \mathrm{CSA} / \mathrm{yr}\left(\mathrm{mm}^{2}\right)$ & $2.0 \pm 2.4 \dagger$ & $-0.7 \pm 3.5$ & $<0.0001$ \\
\hline$\triangle \mathrm{EEM} \mathrm{CSA/yr} \mathrm{( \% )}$ & $8.4 \pm 10.2$ & $-1.6 \pm 9.6 \dagger$ & $<0.0001$ \\
\hline$\Delta$ Lumen CSA/yr $\left(\mathrm{mm}^{2}\right)$ & $1.0 \pm 2.7$ & $-1.2 \pm 1.9^{*}$ & $<0.0001$ \\
\hline$\Delta$ Lumen CSA/yr (\%) & $8.1 \pm 18.5$ & $-7.0 \pm 11.2^{*}$ & $<0.0001$ \\
\hline$\triangle \mathrm{P} \& \mathrm{M}$ CSA/yr $\left(\mathrm{mm}^{2}\right)$ & $1.0 \pm 1.2^{*}$ & $0.6 \pm 1.8 \dagger$ & 0.5 \\
\hline$\triangle \mathrm{P} \& \mathrm{M}$ CSA/yr (\%) & $15.2 \pm 19.2$ & $7.5 \pm 18.4$ & 0.2 \\
\hline \multicolumn{2}{|c|}{ Documented prior serial lesion remodeling } & & $<0.05 \ddagger$ \\
\hline$\triangle \mathrm{EEM} C S A>0$ & $24(89 \%)$ & $11(58 \%)$ & \\
\hline$\triangle \mathrm{EEM} C S A \leq 0$ & $3(11 \%)$ & $8(42 \%)$ & \\
\hline
\end{tabular}

Reference vs. lesion:

${ }^{*} p<0.01$

${ }^{t} p<0.05$

${ }^{\ddagger}$ compared using Fisher exact test.

$\Delta$ = changes; other abbreviations as in Table 2.

Overall, the follow-up RI correlated directly with $\triangle$ lesion-site EEM CSA (baselineto-follow-up) (Figure 1); of note, there was a substantial amount of patient-topatient variation. In addition, there was no correlation between $\Delta$ reference EEM CSA and the follow-up RI $(y=-0.01 x+1.05, r=0.16, p=0.15$; graph not shown), whereas $\triangle$ reference EEM CSA did correlate directly with $\triangle$ lesion EEM CSA $(y=0.41 x+0.82, r=0.53, p<0.001$; graph not shown). 


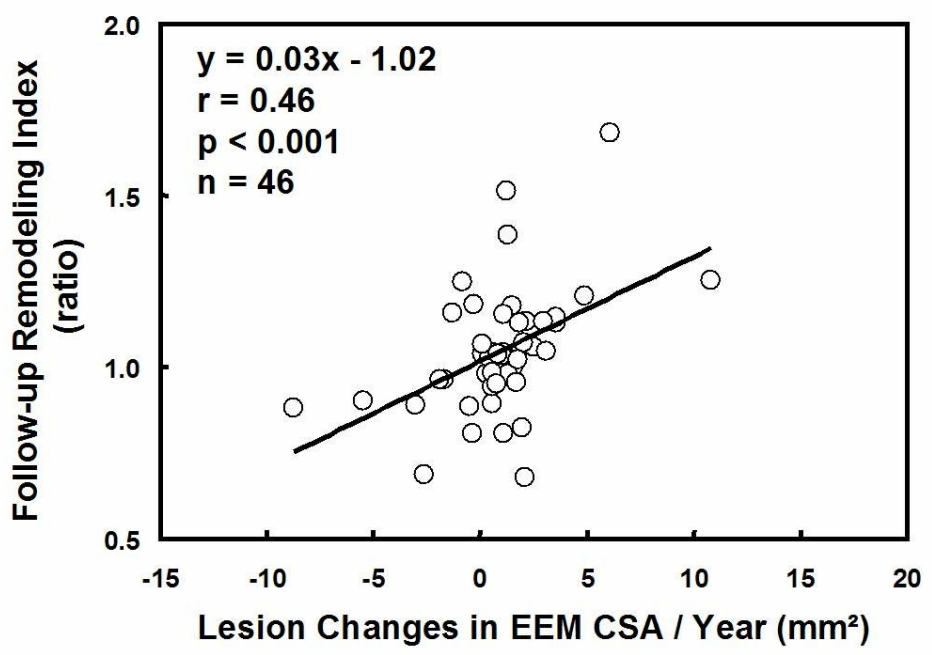

Figure 1. Relation between annual changes in vessel dimensions at lesion site versus the remodeling index at follow-up. Serial (baseline-to-follow-up) changes in external elastic membrane (EEM) cross-sectional area (CSA) correlated with the remodeling index at follow-up.

Using multivariate linear regression analysis, the follow-up $\mathrm{RI}$ was dependent on the baseline RI, the increase in lesion-site EEM CSA, and the decrease in reference EEM CSA. The follow-up RI was not dependent on changes in lesion-site P\&M CSA.

\section{Follow-up versus baseline RI}

There was a significant linear relationship between the follow-up $\mathrm{RI}$ and the baseline $\mathrm{RI}$ (Figure 2). In $74 \%$ of lesions, the classification of remodeling as positive or intermediate/negative was similar at baseline and follow-up (Table 4).

Table 4. Comparison of Positive Versus Intermediate/Negative Remodeling Classification at Baseline and Follow-Up

\begin{tabular}{lccc} 
& $\begin{array}{c}\text { Baseline RI }>1 \\
(\mathbf{n}=29)\end{array}$ & $\begin{array}{c}\text { Baseline } \mathbf{R I} \leq \mathbf{1} \\
(\mathbf{n}=17)\end{array}$ & p Value \\
Follow-up RI $\leq 1(\mathrm{n}=19)$ & $7(24 \%)$ & $12(71 \%)$ & $<0.01^{*}$ \\
Follow-up RI > 1 $(\mathrm{n}=27)$ & $22(76 \%)$ & $5(29 \%)$ & \\
\hline$R I=$ remodeling index * & &
\end{tabular}

$R I=$ remodeling index. ${ }^{*}$ compared using chi-square statistics 


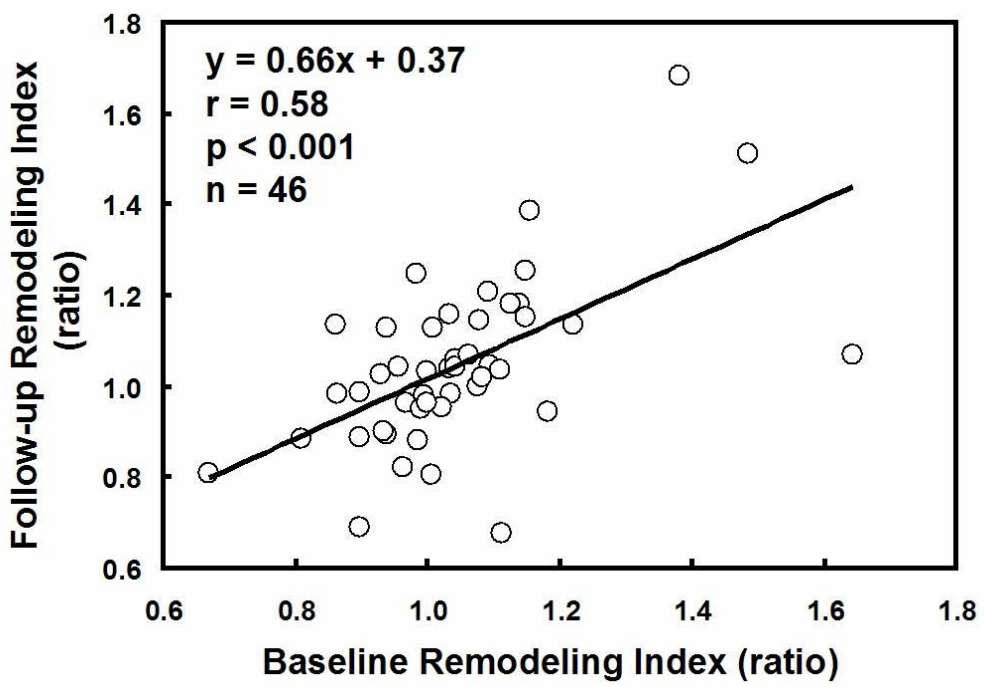

Figure 2. Linear relation between the remodeling index at follow-up versus the remodeling index at baseline.

\section{Discussion}

Positive (or outward) remodeling has been postulated to explain the finding of atherosclerosis that does not encroach on the arterial lumen. Remodeling of atherosclerotic human coronary arteries was described first by Glagov et al. ${ }^{1}$ (primarily in non-stenotic reference segments from autopsied hearts) by correlating EEM CSA with plaque CSA and plaque burden. The observations by Glagov et al. ${ }^{1}$ were confirmed in vivo using IVUS., ${ }^{3,4,13}$ Since then, lesion remodeling has been most often studied by comparing the lesion to proximal and/or distal reference segments at a single point in time to generate an $\mathrm{RI}$, even though these reference segments have also remodeled. ${ }^{13}$ Lesions are classified as positive versus intermediate and negative remodeling based on this comparison. ${ }^{13,14}$ Although there are many theoretical limitations to this approach ${ }^{13}$, the results of our current serial IVUS study show that the RI is a relatively good substitute of true vascular remodeling.

When the baseline-to-follow-up change in EEM CSA was compared to the follow-up RI, lesions with a follow-up RI $>1$ had a previously documented increase in EEM in nearly $90 \%$. The independent predictors of the follow-up RI were the baseline $\mathrm{RI}$, the increase in lesion EEM CSA, and the decrease in reference EEM CSA. Our study demonstrates that the classification of a lesion as positive versus 
intermediate/negative remodeling tends to remain relatively constant over time. We found a significant linear relationship between the follow-up RI versus the baseline $\mathrm{RI}$, and in almost $75 \%$ of lesions, the classification was similar at baseline and after 18 months of follow-up. In particular, positively remodeled lesions continued to positively remodel. We found a significant correlation between changes in lesion EEM CSA (serial remodeling) and the follow-up RI. However, there was a substantial patient-to-patient variation (e.g., the RI varied from approximately 0.7 to 1.5 when in fact there was no serial remodeling) (Figure 1).

Thus, the RI may be a very good correlate of true remodeling for a population rather than for an individual lesion. In addition, our data showed no difference in baseline plaque burden between lesions with positive versus negative RI, thereby questioning the concept that remodeling occurs until approximately $40 \%$ of the artery is occupied by plaque. ${ }^{1}$

\section{IVUS insights into remodeling from previous serial studies}

Few serial IVUS studies in native coronary arteries (like the present study) have been reported. Such studies permit an assessment of remodeling independent of reference segment changes. Shiran et al. $^{15}$ studied 31 left main stems to demonstrate that lumen changes during six months follow-up resulted primarily from EEM changes (i.e., remodeling). Although the assessment of arterial remodeling was not the main purpose of the pharmacological intervention studies by Takagi et al. ${ }^{16}$ and Schartl et al. ${ }^{17}$, the presence of positive remodeling was clearly demonstrated.

\section{Why study arterial remodeling?}

Cumulative evidence from various studies suggests that positively remodeled lesions are more biologically active than intermediate or negatively remodeled lesions and that they occur in patients who are more prone to develop additional unstable lesions or other forms of clinical instability. ${ }^{21,22}$ For instance, de novo coronary lesions in patients with acute coronary syndromes more often have positive remodeling characteristics compared with either chronic stable angina lesions or to control plaques elsewhere in the coronary tree..$^{9,10,12,23}$ In addition, positive remodeling was found to be strongly associated with single and multiple plaque ruptures and thrombus formation in such patients. ${ }^{11,24-26}$ In the context of percutaneous coronary interventions, positive remodeling has been shown to be a predictor of: 1) post-interventional creatinine kinase-MB elevation ${ }^{27}$; 2 ) no reflow after primary infarct angioplasty ${ }^{28}$; 3) recurrent ischemia within one month after thrombolysis for acute myocardial infarction ${ }^{29}$; 4) target lesion revascularization 
after non-stent interventions ${ }^{30}$; 5) major adverse coronary events in patients with unstable angina undergoing percutaneous revascularization ${ }^{31}$; 6) target vessel revascularization and intimal hyperplasia after bare metal stenting ${ }^{32,33}$; 7) intimal hyperplasia after implantation of drug-eluting stents ${ }^{34}$; and 8) in-hospital complications, major adverse coronary events, restenosis, and new lesion formation in patients with stable angina. ${ }^{35}$

\section{Study limitations}

By most standards, this was a large serial IVUS study; however, all long-term serial IVUS studies are limited to a relatively small number of patients. We only studied left main disease as representative of non-intervened coronary segments; therefore, our findings may not be applicable to all (stenotic and non-stenotic) coronary segments. We only included patients who underwent non-left main intervention and who were admitted for repeat cardiac catheterization $\geq 12$ months later; thus, the findings of the current study may not be applicable to the general population. ${ }^{36} \mathrm{We}$ used two IVUS systems; although this approach may have shortcomings, every effort was taken to obtain the most reliable data possible as previously discussed. ${ }^{18}$ Three-dimensional (electrocardiogram-gated) IVUS analyses ${ }^{37}$ may be superior for the assessment of atherosclerotic coronary arteries compared to the twodimensional analysis used in the current study. All patients were Caucasian, and most were men. We only used one classification of remodeling; there are others that have been reported. Of the 60 patients who have been reported previously, only 46 patients could be included in this analysis because of a lack of a welldefined reference segment in the other 14 patients. Our data did not permit the comparison of different definitions of the $\mathrm{RI}^{38}$; therefore, our findings may be limited to the definition used in the present study.

\section{Conclusions}

The vast majority of lesions with positive remodeling classification (RI>1) had evidence of a previous increase in lesion-site EEM CSA.

\section{References}

1. Glagov S, Weisenberg E, Zarins CK, et al. Compensatory enlargement of human atherosclerotic coronary arteries. N Engl J Med 1987;316:1371-1375.

2. Clarkson TB, Prichard RW, Morgan TM, et al. Remodeling of coronary arteries in human and nonhuman primates. JAMA 1994;271:289-294.

3. Pasterkamp G, Wensing PJ, Post MJ, et al. Paradoxical arterial wall shrinkage may contribute to luminal narrowing of human atherosclerotic femoral arteries. Circulation 1995;91:1444-1449. 
4. Ge J, Erbel R, Zamorano J, et al. Coronary arterial remodeling in atherosclerotic disease an intravascular ultrasound study in vivo. Coron Artery Dis 1993;4:981-986.

5. Nishioka T, Luo H, Eigler NL, et al. Contribution of inadequate compensatory enlargement to development of human coronary artery stenosis an in vivo intravascular ultrasound study. J Am Coll Cardiol 1996;27:1571-1576.

6. Mintz GS, Kent KM, Pichard AD, et al. Contribution of inadequate arterial remodeling to the development of focal coronary artery stenoses an intravascular ultrasound study. Circulation 1997;95:1791-1798.

7. von Birgelen C, Airiian SG, Mintz GS, et al. Variations of remodeling in response to left main atherosclerosis assessed with intravascular ultrasound in vivo. Am J Cardiol 1997;80:1408-1413.

8. Weissman NJ, Sheris SJ, Chari R, et al. Intravascular ultrasonic analysis of plaque characteristics associated with coronary artery remodeling. Am J Cardiol 1999;84:37-40.

9. Gyöngyösi M, Yang P, Hassan A, et al. Arterial remodelling of native human coronary arteries in patients with unstable angina pectoris a prospective intravascular ultrasound study. Heart 1999;82:68-74.

10. Schoenhagen $\mathrm{P}$,Ziada KM, Kapadia SR, et al. Extent and direction of arterial remodeling in stable versus unstable coronary syndromes an intravascular ultrasound study. Circulation 2000;101:598603.

11. von Birgelen C, Klinkhart W, Mintz GS, et al. Plaque distribution and vascular remodeling of ruptured and nonruptured coronary plaques in the same vessel an intravascular ultrasound study in vivo. J Am Coll Cardiol 2001;37:1864-1870

12. Nakamura $\mathrm{M}$, Nishikawa $\mathrm{H}$, Mukai $\mathrm{S}$, et al. Impact of coronary artery remodeling on clinical presentation of coronary artery disease an intravascular ultrasound study. J Am Coll Cardiol 2001;37:63-69.

13. Schoenhagen $P$, Ziada KM, Vince DG, et al. Arterial remodeling and coronary artery disease the concept of "dilated" versus "obstructive" coronary atherosclerosis. J Am Coll Cardiol 2001; 38:297306.

14. Mintz GS, Nissen SE, Anderson WD, et al. American College of Cardiology clinical expert consensus document on standards for acquisition, measurement and reporting of intravascular ultrasound studies. J Am Coll Cardiol 2001;37:1478-1492.

15. Shiran A, Mintz GS, Leiboff B, et al. Serial volumetric intravascular ultrasound assessment of arterial remodeling in left main coronary artery disease. Am J Cardiol 1999;83:1427-1432.

16. Takagi T, Yoshida K, Akasaka T, et al. Intravascular ultrasound analysis of reduction in progression of coronary narrowing by treatment with pravastatin. Am J Cardiol 1997;79:1673-1676.

17. Schartl M, Bocksch W, Koschyk DH, et al. Use of intravascular ultrasound to compare effects of different strategies of lipid-lowering therapy on plaque volume and composition in patients with coronary artery disease. Circulation 2001;104:387-392.

18. von Birgelen C, Hartmann M, Mintz GS, et al. Relation between progression and regression of atherosclerotic left main coronary artery disease and serum cholesterol levels as assessed with serial long-term ( $\geq 12$ months) follow-up intravascular ultrasound. Circulation 2003;108:2757-2762.

19. von Birgelen C, Hartmann M, Mintz GS, et al. Spectrum of remodeling behavior observed with serial long-term ( $\geq 12$ months) follow-up intravascular ultrasound studies in left main coronary arteries. Am J Cardiol 2004;93:1107-1113.

20. Baumgart $\mathrm{D}$, Haude $\mathrm{M}, \mathrm{Ge} \mathrm{J}$, et al. Online integration of intravascular ultrasound images into angiographic images. Cathet Cardiovasc Diagn 1996;39:328-329.

21. Pasterkamp G, Schoneveld AH, van der Wal AC, et al. Relation of arterial geometry to luminal narrowing and histologic markers for plaque vulnerability the remodeling paradox. J Am Coll Cardiol 1998;32:655-662.

22. Varnava AM, Mills PG, Davies MJ, et al. Relationship between coronary artery remodeling and plaque vulnerability. Circulation 2002;105:939-943. 
23. Endo A, Hirayama $\mathrm{H}$, Yoshida $\mathrm{O}$, et al. Arterial remodeling influences the development of intimal hyperplasia after stent implantation. J Am Coll Cardiol 2001;37:70-75.

24. Fujii K, Kobayashi Y, Mintz GS, et al. Intravascular ultrasound assessment of ulcerated ruptured plaques a comparison of culprit and nonculprit lesions of patients with acute coronary syndromes and lesions in patients without acute coronary syndromes. Circulation 2003;108:2473-2478.

25. Kotani J, Mintz GS, Castagna MT, et al. Intravascular ultrasound analysis of infarct-related and non-infarct-related arteries in patients who presented with an acute myocardial infarction. Circulation 2003;107:2889-2893.

26. Rioufol G, Finet G, Ginon I, et al. Multiple atherosclerotic plaque rupture in acute coronary syndrome a three-vessel intravascular ultrasound study. Circulation 2002;106:804-808.

27. Mehran R, Dangas G, Mintz GS, et al. Atherosclerotic plaque burden and CK-MB enzyme elevation after coronary interventions intravascular ultrasound study of 2256 patients. Circulation 2002;101:604-610.

28. Watanabe T, Nanto S, Uematsu M, et al. Prediction of no-reflow phenomenon after successful percutaneous coronary intervention in patients with acute myocardial infarction intravascular ultrasound findings. Circ J 2003;67:667-671.

29. Gyöngyösi $M$, Wexberg $P$, Kiss $K$, et al. Adaptive remodeling of the infarct-related artery is associated with recurrent ischemic events after thrombolysis in acute myocardial infarction. Coron Artery Dis 2001;12:167-172.

30. Dangas G, Mintz GS, Mehran R, et al. Preintervention arterial remodeling as an independent predictor of target-lesion revascularization after nonstent coronary intervention an analysis of 777 lesions with intravascular ultrasound imaging. Circulation 1999;99:3149-3154.

31. Gyöngyösi M, Yang P, Hassan A, et al. Intravascular ultrasound predictors of major adverse cardiac events in patients with unstable angina. Clin Cardiol 2000;23:507-515.

32. Okura $\mathrm{H}$, Morino $\mathrm{Y}$, Oshima A, et al. Preintervention arterial remodeling affects clinical outcome following stenting an intravascular ultrasound study. J Am Coll Cardiol 2001;37:1031-1035.

33. Sahara $M$, Kirigaya $H$, Oikawa $Y$, et al. Arterial remodeling patterns before intervention predict diffuse in-stent restenosis an intravascular ultrasound study. J Am Coll Cardiol 2003;42:1731-1738.

34. Mintz GS, Tinana A, Hong MK, et al. Impact of preinterventional arterial remodeling on neointimal hyperplasia after implantation of (non-polymer-encapsulated) paclitaxel-coated stents a serial volumetric intravascular ultrasound analysis from the ASian Paclitaxel-Eluting Stent Clinical trial (ASPECT). Circulation 2003;108:1295-1298.

35. Wexberg P, Gyöngyösi M, Sperker W, et al. Pre-existing arterial remodeling is associated with inhospital and late adverse cardiac events after coronary interventions in patients with stable angina pectoris. J Am Coll Cardiol 2000;36:1860-1869.

36. von Birgelen C, Hartmann M, Mintz GS, et al. Relationship between cardiovascular risk as predicted by established risk scores versus plaque progression as measured by serial intravascular ultrasound. Circulation 2004;110:1579-1585.

37. von Birgelen C, de Vrey EA, Mintz GS, et al. ECG-gated three-dimensional intravascular ultrasound feasibility and reproducibility of an automated analysis of coronary lumen and atherosclerotic plaque dimensions in humans. Circulation 1997;96:2944-2952.

38. Hibi K, Ward MR, Honda Y, et al. Impact of different definitions on the interpretation of coronary remodeling determined by intravascular ultrasound. Catheter Cardiovasc Interv 2005;65:233-239. 


\section{Chapter 8}

Relation Between Baseline Plaque Burden and Subsequent Remodelling of Atherosclerotic Left Main Coronary Arteries: A Serial Intravascular Ultrasound Study with Long-term ( $\geq 12$ Months)

Follow-up

Marc Hartmann, Clemens von Birgelen, Gary S. Mintz, Patrick M.J. Verhorst, Raimund Erbel

Reprinted with permission from:

European Heart Journal 2006 27(15):1778-1784.

(c) 2006 The European Society of Cardiology / Oxford University Press 



\section{Abstract}

Aims: Glagov's histopathological observation and non-serial intravascular ultrasound studies (IVUS) concluded that compensatory coronary remodelling diminishes as $40 \%$ atherosclerotic plaque burden is reached. We tested this hypothesis with serial IVUS.

Methods and Results: Serial IVUS examinations of 46 atherosclerotic non-stenotic left main stems (18 \pm 8 months apart) were analysed to assess the relation between baseline plaque burden (=plaque+media area/vessel area) vs. serial remodelling (=vessel area at baseline-at follow-up). There were 25 plaques with baseline plaque burden $<40 \%(30.1 \pm 6.6 \%$, group $A)$ and 21 plaques with baseline plaque burden $\geq 40 \%(46.1 \pm 5.8 \%$, group $B)$. There was no relation between baseline plaque burden vs. subsequent changes in vessel area overall $(r=0.07, P=0.7)$, for group $A(r=0.03$, $P=0.6)$, and group $B(r=0.01, P=0.8)$. The frequency of positive serial remodelling (vessel area increase) vs. negative or intermediate serial remodelling (no change or decrease) were similar in group A [17 (68\%) vs. $8(32 \%)$ ] and group B lesions [18 (86\%) vs. $3(14 \%)](P=0.2)$.

Conclusion: IVUS demonstrates that serial coronary remodelling is not related to baseline plaque burden. Lesions with baseline plaque burden $<40 \%$ may subsequently show a lack of compensation or frank arterial shrinkage, whereas lesions with baseline plaque burden $>40 \%$ may continue to develop compensatory enlargement.

\section{Introduction}

The concept of positive remodelling - human coronary artery enlargement with increasing atherosclerotic plaque burden to maintain lumen dimensions - was introduced by Glagov et al. ${ }^{1}$ based on their histopathological data. Using regression analysis, they concluded that compensatory remodelling diminished when plaque burden reached a level of $40 \%$ and that plaque progression beyond this threshold lead to lumen narrowing. ${ }^{1}$ Early cross-sectional in vivo intravascular ultrasound studies (IVUS) at a single point in time confirmed these findings. ${ }^{2-5}$ However, all observations at a single point in time have shortcomings in analysing a dynamic process. $^{6-15}$ In fact, direct evidence of remodelling requires serial measurements of total vessel dimensions obtained at two (or more) timepoints. ${ }^{16,18}$ In the present study, we retrospectively analysed serial IVUS data in non-stenotic left main (LM) lesions to evaluate the relation between baseline atherosclerotic plaque burden and serial remodelling (i.e. subsequent changes in lesion site total vessel area).

\section{Methods}

\section{Study population}

We retrospectively analysed serial IVUS data of 46 patients who had haemodynamically non-significant de novo LM atherosclerotic lesions and reliable 
reference segments. These 46 patients were derived from a population of 60 lesions that has previously been reported. ${ }^{10,18-20}$ Fourteen LM lesions were excluded because there was a lack of a well-defined reference. ${ }^{6,10}$ All patients meeting the following criteria were selected for retrospective analysis from our IVUS database: (i) serial high-quality IVUS imaging of the entire LM stem $\geq 12$ months apart; (ii) calcifications that did not limit the quantitative assessment of vessel area (shadowing $\leq 75^{\circ}$ of the adventitial circumference by individual calcific deposits; (iii) non-ostial target site location; (iv) angiographic lumen diameter stenosis $<30 \%$ ('worst view' visual assessment); ( $v$ ) no intervention in the very proximal left anterior descending or circumflex coronary artery segments because these interventions could have affected the LM artery; and (vi) well-defined lesion and reference segment image slices. ${ }^{6}$

Patients were examined in the Essen University Cardiac Catheterization Laboratory with a follow-up of $18 \pm 8$ months (median 15 months, IQR: $12-20$ months). Follow-up IVUS studies (each patient had one follow-up IVUS examination) were performed during clinically driven follow-up catheterization because of (i) repeat interventions of a (non-LM) coronary stenosis; (ii) reevaluation of the results of interventional procedures; and/or (iii) clinical symptoms such as progression of angina pectoris (not related to significant LM stem disease). Differences in the timing of follow-up catheterization were not related to the target of the present study - LM coronary artery disease (CAD). The IVUS study was approved by the Local Council on Human Research. All patients signed a written informed consent form as approved by the Local Medical Ethics Committee.

\section{Demographics, medication, and lipid profile}

Demographics including cardiovascular risk factors, medications, and lipid profiles were recorded including diabetes mellitus and hypertension (medicationdependent); hypercholesterolaemia [medication-dependent, total serum cholesterol $>200 \mathrm{mg} / \mathrm{dL}$, or low-density lipoprotein (LDL) cholesterol $>160 \mathrm{mg} / \mathrm{dL}$; ; history of smoking, and family history of CAD. Data of laboratory tests were the mean of the baseline and follow-up values. Plasma concentrations of total cholesterol, LDL cholesterol, high-density lipoprotein (HDL) cholesterol, and triglycerides were measured by standard enzymatic methods. Medication was recorded only if drugs were taken for $>50 \%$ of the follow-up interval (e.g. clopidogrel for 4 weeks was not tabulated).

\section{IVUS imaging}

IVUS was performed as previously described. ${ }^{10,18}$ In brief, IVUS studies were 
performed during percutaneous coronary interventions of mid or distal left anterior or left circumflex arteries after intracoronary injections of $200 \mu \mathrm{g}$ nitroglycerin. Two commercial systems were used: a mechanical sector scanner (Boston Scientific Corporation, San Jose, CA, USA) incorporating a $30 \mathrm{MHz}$ single-element bevelled transducer or a solid-state device (Endosonics, Rancho Cordova, CA, USA). Slow continuous pullbacks of the IVUS transducer were started as distal as possible in one of the left coronary arteries and were generally performed using a motorized pullback device (at $0.5 \mathrm{~mm} / \mathrm{s}$ ). IVUS images of the entire pullback were recorded on $0.5 \mathrm{in}$. high-resolution s-VHS tape for off-line analysis. In addition, a dedicated image-in image system (Echo-Map, Siemens, Erlangen, Germany) ${ }^{21}$ was used to record the 'angiographic' position of the IVUS probe together with the corresponding IVUS image - especially, at sites of characteristic landmarks (i.e. calcifications or unusual plaque shapes) and/or the target site. Importantly, at Essen University, if a patient undergoes imaging with one IVUS system during an index procedure, the same IVUS system is used at follow-up.

\section{IVUS analysis}

The target lesion site image slice was the slice with the smallest baseline lumen area. ${ }^{18}$ If there were several slices with equal lumen size, the one with the largest total vessel and plaque+media (=total vessel minus lumen area) area was analysed. ${ }^{18}$ A LM stem reference image slice was selected as the most normallooking cross-section (largest lumen with smallest plaque+media area) distal or proximal to the target lesion. ${ }^{10}$ Exact matching of the initial and follow-up IVUS studies was ensured using side-by-side comparison of the serial IVUS video sequences along with information of the pullback speed; ${ }^{6}$ the operators' recorded comments (on video tape); and characteristic calcifications, vascular and perivascular landmarks, and plaque shapes. If required, the angiographic sequences of the dedicated image-in-image system (Echo-Map) were revisited to optimize matching. ${ }^{21}$

The lumen area was measured by tracing the leading edge of the intima. The total vessel area was measured by tracing the leading edge of the adventitia. As in many previous IVUS studies, plaque+media was used as a measure of atherosclerotic plaque because IVUS cannot measure media thickness accurately. ${ }^{6}$ Plaque burden (\%) was calculated as (plaque+media divided by total vessel area) $\times 100 \%$. In our laboratory, the intraclass correlation coefficient is 0.99 for repeated measurements of total vessel, 0.96 for lumen, and 0.99 for plaque+media area. We used a cut-off value of $40 \%$ to compare the baseline and serial remodelling of lesions with a baseline plaque burden $<40 \%$ (group A) vs. $\geq 40 \%$ (group B). Rationale for using this cut-off value, as introduced by Glagov et al. 
based on their regression analyses of histopathological data, was the fact that this value was considered to be a threshold above which no compensatory coronary remodelling occurs. ${ }^{1}$

Plaque composition was assessed visually as previously described. ${ }^{6}$ The arc of calcium was measured with a protractor centred on the lumen; if necessary, the total arc of calcium was obtained by adding arcs of individual deposits. As in previous IVUS studies, ${ }^{18,19,22}$ plaques were classified as calcified if the total arc of lesion calcium was $>180^{\circ}$. Extrapolation of the total vessel area boundary behind calcium was possible, if each individual calcific deposit did not shadow $>75^{\circ}$ of the adventitial border. ${ }^{18}$

We calculated the changes between the initial and follow-up IVUS measurements. To compensate for the variation in follow-up interval and to obtain comparable data, measurements were normalized for the length of the follow-up period; and annual changes (i.e. changes per year) were calculated (normalized area changes=individual area changes divided by the individual follow-up period in months multiplied by 12), reported and compared.

The baseline remodelling index $(\mathrm{RI})$ was calculated as lesion site divided by reference total vessel area. ${ }^{6,10}$ As in previous studies, a remodelling index $>1$ defined positive baseline remodelling and a remodelling index $\leq 1$ intermediate/negative baseline remodelling. ${ }^{6,8-10}$ The change in lesion site total vessel area during follow-up was the measure of serial arterial remodelling. As in previous studies, changes in total vessel area $>0$ defined positive serial remodelling and changes in total vessel area $\leq 0$ defined intermediate/negative serial remodelling. ${ }^{6,10,16-18}$

\section{Statistical analysis}

Analyses were performed with SPSS 13.0 (SPSS Inc., Chicago, IL, USA). Dichotomous data are presented as frequencies and compared using $\lambda^{2}$ statistics or Fisher's exact test when at least $25 \%$ of values showed an expected frequency less than 5. Quantitative data are presented as mean \pm 1 SD and compared using Student's t-test and linear regression analysis. Two-sided P-values of less than 0.05 were considered significant. The alpha-level was not adjusted for multiple testing, as we did not perform multiple testing. 


\section{Results}

Demographics, medications, and lipid profile

There were 25 plaques with a baseline plaque burden $<40 \%$ (group A) and 21 plaques with a baseline plaque burden $\geq 40 \%$ (group B). Comparing these two groups, there were no differences in demographics, medications, and lipid profiles with the exception of more ACE-inhibitor use in group B (Table 1).

Table 1(part 1). Demographics, medication, and lipid profile

\begin{tabular}{|c|c|c|c|}
\hline & $\begin{array}{c}\text { Group } A: \\
\text { Plaque burden } \\
<40 \% \\
(n=25)\end{array}$ & $\begin{array}{c}\text { Group B : } \\
\text { Plaque burden } \\
\geq 40 \% \\
(n=21)\end{array}$ & $\mathbf{P}$ \\
\hline Time of follow-up (months) & $18.8 \pm 9.6$ & $17.6 \pm 6.2$ & 0.9 \\
\hline Age (years) & $56 \pm 7$ & $60 \pm 9$ & 0.2 \\
\hline Men & $22(88)$ & $19(90)$ & 0.6 \\
\hline Body mass index $\left(\mathrm{kg} / \mathrm{m}^{2}\right)$ & $26 \pm 2$ & $27 \pm 5$ & 0.2 \\
\hline Hypercholesterolemia,n (\%) & $23(92)$ & $18(86)$ & 0.6 \\
\hline Systemic arterial hypertension, $\mathrm{n}(\%)$ & $17(68)$ & $17(81)$ & 0.5 \\
\hline Diabetes mellitus, $\mathrm{n}(\%)$ & $1(4)$ & $5(24)$ & 0.1 \\
\hline Smoker, n (\%) & $9(36)$ & $6(29)$ & 0.6 \\
\hline Family history of coronary disease, $\mathrm{n}(\%)$ & $6(27)$ & $4(19)$ & 0.7 \\
\hline Previous myocardial infarction n (\%) & $8(32)$ & $6(29)$ & 0.8 \\
\hline One vessel narrowed > 50\%, n (\%) & $13(52)$ & $7(33)$ & 0.4 \\
\hline Two vessel narrowed $>50 \%, n(\%)$ & $6(24)$ & $7(33)$ & \\
\hline Three vessel narrowed $>50 \%, n(\%)$ & $6(24)$ & $7(33)$ & \\
\hline Stable angina CCS class I, n (\%) & $7(28)$ & $8(38)$ & 0.9 \\
\hline Stable angina CCS class II, $\mathrm{n}(\%)$ & $11(44)$ & $7(33)$ & \\
\hline Stable angina CCS class III, n (\%) & $5(20)$ & $4(19)$ & \\
\hline Unstable angina, $\mathrm{n}(\%)$ & $2(8)$ & $2(10)$ & \\
\hline
\end{tabular}

CCS, Canadian Cardiovascular Society 
Table 1(part 2). Demographics, medication, and lipid profile

\begin{tabular}{|c|c|c|c|}
\hline & $\begin{array}{c}\text { Group A : } \\
\text { Plaque burden } \\
<40 \% \\
(n=25)\end{array}$ & $\begin{array}{c}\text { Group B : } \\
\text { Plaque burden } \\
\geq 40 \% \\
(n=21)\end{array}$ & $\mathbf{P}$ \\
\hline \multicolumn{4}{|l|}{ Medication } \\
\hline Acetylsalicylic acid, n (\%) & $25(100)$ & $21(100)$ & 1.0 \\
\hline ACE inhibitors, $n(\%)$ & $5(20)$ & $13(62)$ & $<0.004$ \\
\hline Angiotensin receptor antagonists, $\mathrm{n}(\%)$ & $0(0)$ & $1(5)$ & 0.5 \\
\hline ß-Blockers, n (\%) & $13(52)$ & $11(52)$ & 1.0 \\
\hline Calcium channel blockers, $\mathrm{n}(\%)$ & $10(40)$ & $5(24)$ & 0.3 \\
\hline Diuretics, $\mathrm{n}(\%)$ & $5(20)$ & $8(38)$ & 0.2 \\
\hline Fibrates, n (\%) & $1(4)$ & $2(10)$ & 0.6 \\
\hline Insulin, $\mathrm{n}(\%)$ & $0(0)$ & $1(5)$ & 0.5 \\
\hline Nitrates, $\mathrm{n}(\%)$ & $16(64)$ & $13(62)$ & 0.9 \\
\hline Oral antidiabetics, $\mathrm{n}(\%)$ & $0(0)$ & $3(14)$ & 0.1 \\
\hline Statins, $\mathrm{n}(\%)$ & $20(80)$ & $16(76)$ & 0.5 \\
\hline \multicolumn{4}{|l|}{ Lipid profile } \\
\hline Total cholesterol (mg/dL) & $189 \pm 37$ & $201 \pm 36$ & 0.3 \\
\hline LDL cholesterol (mg/dL) & $118 \pm 41$ & $124 \pm 50$ & 0.6 \\
\hline HDL cholesterol (mg/dL) & $45 \pm 9$ & $51 \pm 17$ & 0.2 \\
\hline Triglycerides (mg/dl) & $136 \pm 76$ & $114 \pm 59$ & 0.3 \\
\hline
\end{tabular}

ACE, angiotensin-converting enzyme; $H D L$, high-density lipoprotein; $L D L$, low-density lipoprotein.

\section{Baseline IVUS data and Glagovian analysis}

Baseline IVUS data are presented in Table 2. In keeping with the definitions, group $\mathrm{B}$ plaques had a greater baseline plaque burden than group $\mathrm{A}(P<0.0001)$. Because there is no difference in total vessel area, lumen area was significantly smaller in group B plaques when compared with group A plaques $(P<0.0004)$. Plaque composition including the total arc of calcium did not differ between the two groups (Table 2). Using baseline IVUS measurements, it was possible to reproduce the linear relationship between plaque and vessel size as well as between plaque burden and lumen size that was first shown by Glagov et al. ${ }^{1}$ (Figure 1). There was a significant positive relation between plaque+media vs. total vessel area suggesting compensatory enlargement to accommodate for an increase in plaque size. There was a significant negative correlation between lumen area vs. plaque burden in lesions with plaque burden $\geq 40 \%$ (group B), but no relationship between lumen area vs. plaque burden in lesions with plaque burden $<40 \%$ (group $A$ ) or in the entire study population ( $r=-0.18, P=0.4$; data not shown) (Figure 1 ) suggesting a stop of compensatory enlargement beyond a plaque burden of $40 \%$. 
Table 2. Baseline intravascular ultrasound data

\begin{tabular}{|c|c|c|c|}
\hline & $\begin{array}{c}\text { Group } A: \\
\text { Plaque burden } \\
<40 \% \\
(n=25)\end{array}$ & $\begin{array}{c}\text { Group B : } \\
\text { Plaque burden } \\
\geq 40 \% \\
(n=21)\end{array}$ & $\mathbf{P}$ \\
\hline \multicolumn{4}{|l|}{ Lesion } \\
\hline Total vessel area $\left(\mathrm{mm}^{2}\right)$ & $24.8 \pm 5.8$ & $25.0 \pm 5.3$ & 0.9 \\
\hline Lumen area $\left(\mathrm{mm}^{2}\right)$ & $17.3 \pm 3.9$ & $13.4 \pm 2.8$ & $<0.0004$ \\
\hline Plaque+media area $\left(\mathrm{mm}^{2}\right)$ & $7.6 \pm 2.8$ & $11.6 \pm 3.4$ & $<0.0001$ \\
\hline Plaque burden $(\%)$ & $30.1 \pm 6.6$ & $46.1 \pm 5.8$ & $<0.0001$ \\
\hline Total arc of caclium $\left(^{\circ}\right)$ & $75 \pm 97$ & $77 \pm 100$ & 0.9 \\
\hline \multicolumn{4}{|l|}{ Lesion plaque composition } \\
\hline Soft, n (\%) & $8(32)$ & $7(33)$ & 0.8 \\
\hline Fibrous, n (\%) & $8(32)$ & $9(43)$ & \\
\hline Calcified, n (\%) & $6(24)$ & $4(19)$ & \\
\hline Mixed, n (\%) & $3(12)$ & $1(5)$ & \\
\hline \multicolumn{4}{|l|}{ Reference } \\
\hline Total vessel area $\left(\mathrm{mm}^{2}\right)$ & $24.4 \pm 7.1$ & $24.6 \pm 7.4$ & 1.0 \\
\hline Lumen area $\left(\mathrm{mm}^{2}\right)$ & $19.6 \pm 5.4$ & $17.8 \pm 5.8$ & 0.3 \\
\hline Plaque+media area $\left(\mathrm{mm}^{2}\right)$ & $4.9 \pm 2.3$ & $6.7 \pm 4.2$ & 0.1 \\
\hline Plaque burden (\%) & $18.7 \pm 6.2$ & $26.4 \pm 11.5$ & $<0.01$ \\
\hline \multicolumn{4}{|l|}{ Lesion baseline remodeling } \\
\hline Remodeling index (ratio) & $1.04 \pm 0.2$ & $1.05 \pm 0.2$ & 0.9 \\
\hline Remodeling index $>1, n(\%)$ & $16(64)$ & $13(62)$ & 0.9 \\
\hline Remodeling index $\leq 1, \mathrm{n}(\%)$ & $9(36)$ & $8(38)$ & \\
\hline
\end{tabular}

\section{Baseline remodelling index}

There was no difference in baseline remodelling index or in the categorization of lesions as baseline positive vs. baseline intermediate/negative remodelling when comparing lesions with a plaque burden $\geq 40 \%$ vs. lesions with a plaque burden $<40 \%$ (Table 2). For all 46 patients (Figure 2) as well as for group A or group B lesions separately ( $r=0.03, P=0.6$, and $r=0.01, P=0.8$, respectively; data not shown), there was no relation between baseline remodelling index vs. baseline plaque burden, which disagrees with the hypothesis of Glagov et al. ${ }^{1}$ References of group B plaques showed a greater plaque burden $(P<0.01)$, but total vessel and lumen area did not differ significantly between both groups (Table 2). 

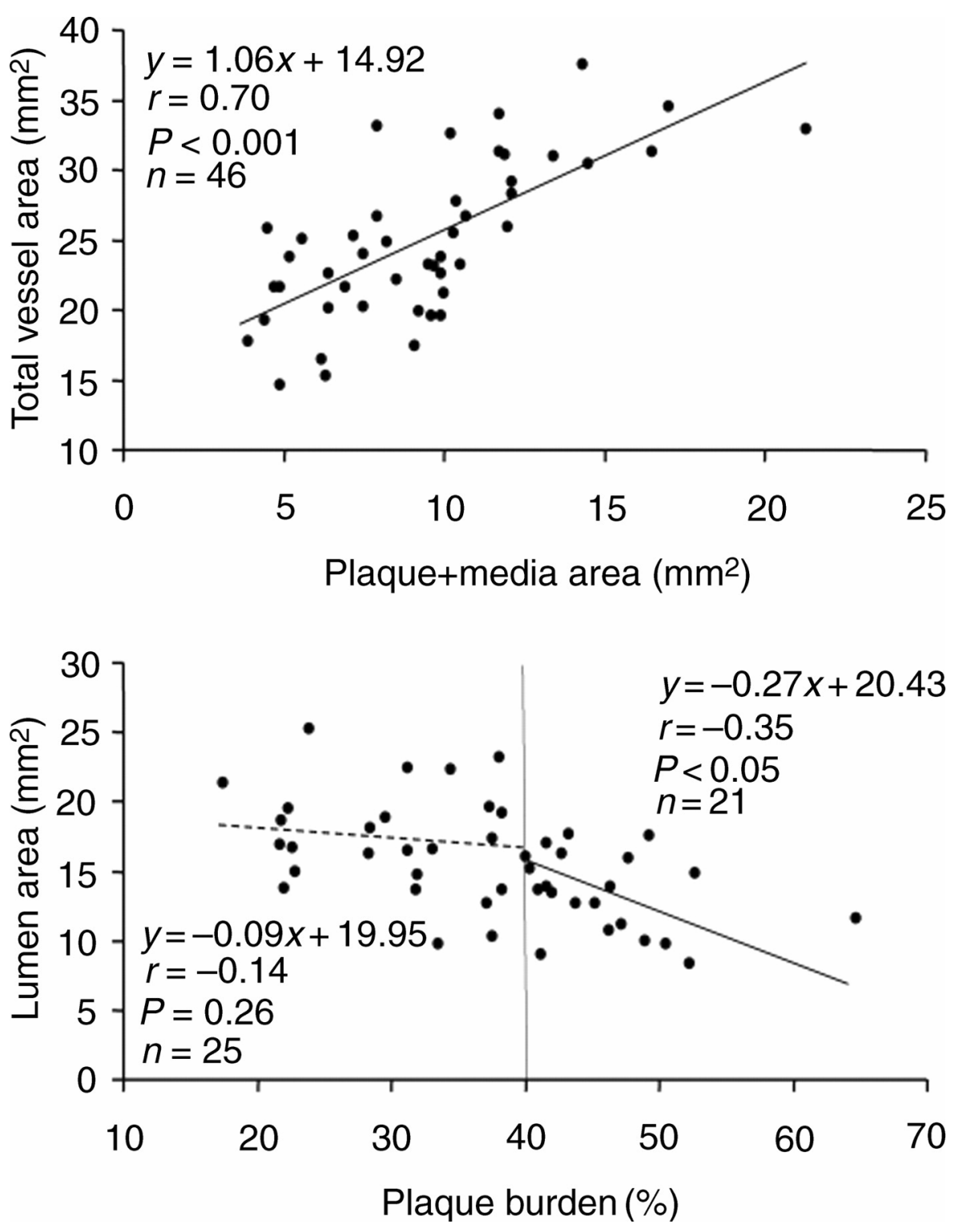

Figure 1. Regression analyses following Glagov et al. ${ }^{1}$ with baseline IVUS data. Upper panel: There was a positive linear relation between plaque+media and total vessel area ('compensatory enlargement hypothesis'). Lower panel: There was a negative linear relation between baseline plaque burden and lumen area only in plaques with plaque burden $>40 \%$ ('compensatory cut-off hypothesis'). 

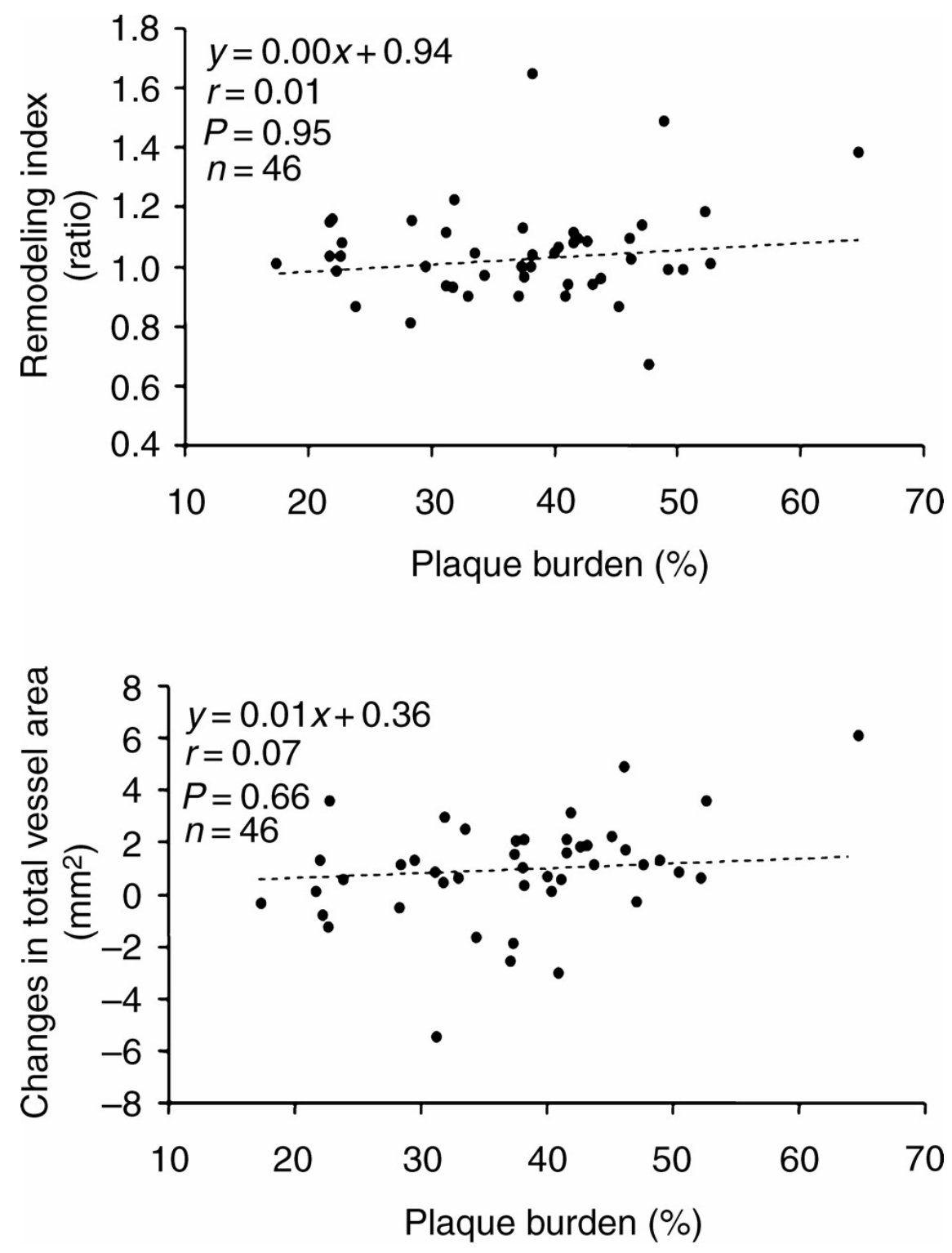

Figure 2. Relation between baseline plaque burden vs. baseline remodelling index and changes in total vessel area (serial remodelling). There was no relation between plaque burden vs. baseline remodelling index (upper panel) and changes in total vessel area (lower panel). 
Serial IVUS data

Between groups $A$ and $B$, there were no significant differences in the magnitude or the direction (increase or decrease) of the annual changes in total vessel or lumen area; in other words, there were no differences in serial remodelling between the two groups (Table 3). There was also no correlation between the change in total vessel area (i.e. serial remodelling) vs. the baseline plaque burden for all 46 patients (Figure 2) or for group A and group B plaques, separately $(r=0.03, P=0.6$, and $\mathrm{r}=0.01, P=0.8$, respectively; data not shown). In fact, changes in total vessel area were flat across the entire range of values of baseline plaque burden, indicating no relation between baseline plaque burden and serial remodelling, which also contradicts the hypothesis of Glagov et al. ${ }^{1}$ In addition, there was no relation between the change in lumen area vs. the baseline plaque burden for all 46 patients ( $\mathrm{r}=0.19, P=0.7$; data not shown) and for group $A$ and group $B$ separately ( $\mathrm{r}=0.25, P=0.2$, and $\mathrm{r}=-0.13, P=0.6$, respectively; data not shown).

Table 3. Serial intravascular ultrasound data

\begin{tabular}{|c|c|c|c|}
\hline & $\begin{array}{c}\text { Group A : } \\
\text { Plaque burden } \\
<40 \% \\
(n=25)\end{array}$ & $\begin{array}{c}\text { Group B : } \\
\text { Plaque burden } \\
\geq 40 \% \\
(n=21)\end{array}$ & $\mathbf{P}$ \\
\hline \multicolumn{4}{|l|}{ Lesion changes } \\
\hline Changes in total vessel area/year $\left(\mathrm{mm}^{2}\right)$ & $0.7 \pm 2.9$ & $1.1 \pm 2.9$ & 0.9 \\
\hline Changes in total vessel area/year (\%) & $3.9 \pm 12.0$ & $4.7 \pm 10.1$ & 0.8 \\
\hline Changes in lumen area/year $\left(\mathrm{mm}^{2}\right)$ & $-0.4 \pm 2.7$ & $0.6 \pm 2.5$ & 0.2 \\
\hline Changes in lumen area/year (\%) & $-1.6 \pm 15.7$ & $6.0 \pm 18.9$ & 0.1 \\
\hline Changes in plaque + media area/year $\left(\mathrm{mm}^{2}\right)$ & $1.1 \pm 1.4$ & $0.5 \pm 1.5$ & 0.1 \\
\hline Changes in plaque+media area/year (\%) & $17.9 \pm 21.6$ & $4.9 \pm 12.7$ & $<0.01$ \\
\hline \multicolumn{4}{|l|}{ Serial lesion remodeling } \\
\hline Changes in total vessel area $>0, n(\%)$ & $17(68)$ & $18(86)$ & 0.2 \\
\hline Changes in total vessel area $\leq 0, \mathrm{n}(\%)$ & $8(32)$ & $3(14)$ & \\
\hline
\end{tabular}

\section{Discussion}

Our serial IVUS data showed that serial coronary arterial remodelling (changes in total vessel area) was not related to baseline plaque burden in moderately diseased LM coronary arteries. Serial changes in vessel area were flat across the entire range of baseline plaque burden values (17.4-64.7\%). Between lesions with a baseline plaque burden $<40$ vs. $\geq 40 \%$, there was no difference in the frequency 
of positive and negative serial remodelling - i.e. the increase or decrease in vessel area.

Glagov et al. ${ }^{1}$ observed a positive correlation between total vessel area and plaque area in a histopathological study of 136 LM coronary arteries; he concluded that this indicated a compensatory enlargement of the vessel during atherogenesis. There was a negative correlation between plaque burden and lumen area in lesions with $\geq 40 \%$ plaque burden, but not in lesions with $<40 \%$ plaque burden. ${ }^{1}$ Thus, a biphasic course of lesion formation was postulated: (i) early preservation of lumen dimensions up to a plaque burden of $40 \%$ and (ii) luminal narrowing as plaque burden exceeded $40 \%$. In the present analysis, we reproduced these relationships using linear regression analysis of baseline IVUS data. However, as baseline lumen area is arithmetically related to baseline plaque burden (plaque burden=total vessel minus lumen divided by total vessel), the use of regression analysis of related variables to analyse different coronary remodelling patterns has theoretical limitations, as has previously been discussed. ${ }^{2,23}$ Furthermore, previous studies have reported a baseline remodelling index $<1.0$ (evidence of intermediate or negative baseline remodelling) in lesions with plaque burden $<40 \% .{ }^{9}$ In the present analysis, the baseline remodelling index was not related to baseline plaque burden, and the frequencies of positive vs. intermediate or negative baseline remodelling did not differ between plaques with a baseline plaque burden $<40$ vs. $\geq 40 \%$.

Recently, we demonstrated that a baseline remodelling index correlated with serial remodelling (subsequent changes in total vessel area). ${ }^{10}$ However, for various reasons (e.g. limitations of a single time-point observation, reference segment selection, vessel tapering, plaque burden, and remodelling of the reference), these baseline indices are acknowledged to be only indirect evidence of serial remodelling. ${ }^{6,9}$ Direct evidence of remodelling requires serial vessel area measurements obtained at two (or more) time-points, as progression of atherosclerosis and changes in vessel dimension are both dynamic processes. ${ }^{2,6,7}$ Few studies have assessed coronary arterial remodelling using serial IVUS measurements. ${ }^{16-18}$ Such studies permit the assessment of remodelling independent of potential changes of the reference segment. ${ }^{6,10,16-18}$ Shiran et al. ${ }^{16}$ studied 31 moderately diseased (mean plaque burden $<40 \%$ ) LM stems and demonstrated that lumen changes during 6 months follow-up resulted primarily from changes in total vessel dimensions - i.e. serial remodelling. In the data presented by Schartl et al. ${ }^{17}$, serial remodelling during 12 months IVUS follow-up did not differ between lesion with a baseline plaque burden $<45$ and $\geq 45 \%$. In the present study, we demonstrated that magnitude and direction of the changes in total vessel area (the magnitude and direction of serial remodelling) were similar in plaques with a baseline plaque burden $<40$ vs. $\geq 40 \%$. Thus, lesions with mild 
baseline plaque burden can either continue to compensate for continued plaque progression to preserve lumen dimensions (i.e. there can be additional serial positive remodelling) or these lesions may develop luminal stenosis as a result of either plaque progression without serial positive remodelling or shrinkage of the total vessel area (negative serial remodelling). ${ }^{10,16}$ Induction of plaque regression is a major target of pharmacological interventions. ${ }^{24,25}$ Treatment with multiple pharmacological agents (e.g. aggressive lipid lowering) may affect serial coronary remodelling (e.g. reverse remodelling) as well as plaque progressionregression. ${ }^{26,27}$

\section{Limitations}

As with most long-term serial IVUS studies, the current report included a relatively small number of patients. We only included patients who underwent non-LM intervention and who were admitted for repeat cardiac catheterization $\geq 12$ months later; thus, the findings of the current study may not be applicable to the general population and other vessels. We only studied mild to moderate LM disease as representatives of non-intervened coronary segments; therefore, our findings may not be applicable to all (stenotic and non-stenotic) coronary segments. The range of plaque burden was somewhat limited (mean: $37.4 \pm 10.2 \%$, median: $38.2 \%$, range: 17.4-64.7\%), however, serial IVUS assessment of significant luminal stenoses is difficult, as such lesions are generally stented and thus not available for follow-up. Heavily calcified lesions were excluded because ultrasound cannot penetrate calcified tissue to measure total vessel area (and remodelling) accurately. ${ }^{6}$ We used two IVUS systems; although this approach may have shortcomings, every effort was taken to obtain the most reliable data possible as previously discussed. ${ }^{10,18}$ Three-dimensional (electrocardiogram-gated) IVUS analyses may be superior for the assessment of atherosclerotic coronary arteries when compared with the two-dimensional analysis used in the current study. ${ }^{28}$ Of the 60 patients that were reported previously, only 46 patients could be included in the present analysis because of a lack of a well-defined reference segment in the other 14 patients. ${ }^{10,18}$ Intensive medical treatment (e.g. statins) may influence the remodelling process. ${ }^{17,26,27}$ Our data used only one definition of the baseline remodelling index; therefore, our findings may not apply to other definitions. ${ }^{8}$ In populations of more advanced atherosclerotic lesions, a predominance of soft plaque composition in lesions with positive remodelling has been shown. ${ }^{29}$ As previously reported, we could not demonstrate a relation between plaque composition and serial remodelling in our population. ${ }^{18}$ In addition, the IVUS analysis of coronary plaque composition based on radiofrequency data ('virtual histology') may be superior to visual assessment of plaque composition. ${ }^{30}$ 


\section{Conclusions}

This IVUS study demonstrates that serial coronary remodelling is not related to baseline plaque burden. Subsequent changes in vessel area are flat across a wide range of baseline plaque burdens. Arterial shrinkage can occur in lesions with baseline plaque burden $<40 \%$, whereas lesions with baseline plaque burden $>40 \%$ may continue to develop compensatory enlargement.

\section{Acknowledgements}

The authors thank Dr Job van der Palen (Medisch Spectrum Twente, Enschede, The Netherlands) for statistical advice.

\section{References}

1. Glagov S, Weisenberg E, Zarins CK, et al. Compensatory enlargement of human atherosclerotic coronary arteries. N Engl J Med 1987;316:1371-1375.

2. Hermiller JB, Tenaglia AN, Kisslo KB, et al. In vivo validation of compensatory enlargement of atherosclerotic coronary arteries. Am J Cardiol 1993;71:665-668.

3. Losordo DW, Rosenfield K, Kaufman J, et al. Focal compensatory enlargement of human arteries in response to progressive atherosclerosis. In vivo documentation using intravascular ultrasound. Circulation 1994;89:2570-2577.

4. Ge J, Erbel R, Zamorano J, et al. Coronary artery remodeling in atherosclerotic disease: an intravascular ultrasonic study in vivo. Coron Artery Dis 1993;4:981-986.

5. Gerber TC, Erbel R, Gorge G, et al. Extent of atherosclerosis and remodeling of the left main coronary artery determined by intravascular ultrasound. Am J Cardiol 1994;73:666-671.

6. Mintz GS, Nissen SE, Anderson WD, et al. American College of Cardiology clinical expert consensus document on standards for acquisition, measurement and reporting of intravascular ultrasound studies (IVUS). J Am Coll Cardiol 2001;37:1478-1492.

7. Weissman NJ. Vascular remodeling: do we really need yet another study? J Am Coll Cardiol 2003;42:811-813.

8. Hibi K, Ward MR, Honda Y, et al. Impact of different definitions on the interpretation of coronary remodeling determined by intravascular ultrasound. Catheter Cardiovasc Interv 2005;65:233-239.

9. Hong MK, Mintz GS, Lee CW, et al. Intravascular ultrasound assessment of patterns of arterial remodeling in the absence of significant reference segment plaque burden in patients with coronary artery disease. J Am Coll Cardiol 2003;42:806-810.

10. von Birgelen C, Hartmann M, Mintz GS, et al. Remodeling index compared to actual vascular remodeling in atherosclerotic left main coronary arteries as assessed with long-term $\geq 12$ months) serial intravascular ultrasound. J Am Coll Cardiol 2006;47:1363-1368.

11. von Birgelen C, Mintz GS, de Vrey EA, et al. Atherosclerotic coronary lesions with inadequate compensatory enlargement have smaller plaque and vessel volumes: observations with three dimensional intravascular ultrasound in vivo. Heart 1998;79:137-142.

12. Nishioka $\mathrm{T}$, Luo $\mathrm{H}$, Eigler $\mathrm{NL}$, et al. Contribution of inadequate compensatory enlargement to development of human coronary artery stenosis: an in vivo intravascular ultrasound study. J Am Coll Cardiol 1996;27:1571-1576. 
13. Smits PC, Bos L, Quarles van Ufford MA, et al. Shrinkage of human coronary arteries is an important determinant of de novo atherosclerotic luminal stenosis: an in vivo intravascular ultrasound study. Heart 1998;79:143-147.

14. Mintz GS, Kent KM, Pichard AD, et al. Contribution of inadequate arterial remodeling to the development of focal coronary artery stenoses: an intravascular ultrasound study. Circulation 1997;95:1791-1798.

15. Wong $\mathrm{CB}$, Porter TR, Xie F, et al. Segmental analysis of coronary arteries with equivalent plaque burden by intravascular ultrasound in patients with and without angiographically significant coronary artery disease. Am J Cardiol 1995;76:598-601.

16. Shiran A, Mintz GS, Leiboff $B$, et al. Serial volumetric intravascular ultrasound assessment of arterial remodeling in left main coronary artery disease. Am J Cardiol 1999;83:1427-1432.

17. Schartl M, Bocksch W, Fateh-Moghadam S. Effects of lipid-lowering therapy on coronary artery remodeling. Coron Artery Dis 2004;15:45-51.

18. von Birgelen C, Hartmann M, Mintz GS, et al. Spectrum of remodeling behavior observed with serial long-term ( $\geq 12$ months) follow-up intravascular ultrasound studies in left main coronary arteries. Am J Cardiol 2004;93:1107-1113.

19. von Birgelen C, Hartmann M, Mintz GS, et al. Relation between progression and regression of atherosclerotic left main coronary artery disease and serum cholesterol levels as assessed with serial long-term ( $\geq 12$ months) follow-up intravascular ultrasound. Circulation 2003;108:2757-2762.

20. von Birgelen C, Hartmann M, Mintz GS, et al. Relationship between cardiovascular risk as predicted by established risk scores vs. plaque progression as measured by serial intravascular ultrasound in left main coronary arteries. Circulation 2004;110:1579-1585.

21. Baumgart $D$, Haude $D, G e J$, et al. Online integration of intravascular ultrasound images into angiographic images. Cathet Cardiovasc Diagn 1996;39:328-329.

22. von Birgelen $C$, Klinkhart W, Mintz GS, et al. Plaque distribution and vascular remodeling of ruptured and nonruptured coronary plaques in the same vessel: an intravascular ultrasound study in vivo. J Am Coll Cardiol 2001;37:1864-1870.

23. von Birgelen C, Airiian SG, Mintz GS, et al. Variations of remodeling in response to left main atherosclerosis assessed with intravascular ultrasound in vivo. Am J Cardiol 1997;80:1408-1413.

24. Nissen SE, Tuzcu EM, Schoenhagen P, et al. Effect of intensive compared with moderate lipidlowering therapy on progression of coronary atherosclerosis. A randomized controlled trial. JAMA 2004;291:1071-1080.

25. Nissen SE, Tuzcu EM, Libby P, et al. Effect of antihypertensive agents on cardiovascular events in patients with coronary disease and normal blood pressure: the CAMELOT study: a randomized controlled trial. JAMA 2004;292:2217-2225.

26. Jensen LO, Thayssen P, Pederson KE, et al. Regression of coronary atherosclerosis by simvastatin: a serial intravascular ultrasound study. Circulation 2004;110:265-270.

27. Sipahi I, Tuzcu EM, Schoenhagen $P$, et al. Paradoxical increase in lumen size during progression of coronary atherosclerosis: observations from the REVERSAL trial. Atherosclerosis 2006;189:229235.

28. von Birgelen C, de Vrey EA, Mintz GS, et al. ECG-gated three-dimensional intravascular ultrasound: feasibility and reproducibility of an automated analysis of coronary lumen and atherosclerotic plaque dimensions in humans. Circulation 1997;96:2944-2952.

29. Sabate M, Kay IP, de Feyter PJ, et al. Remodeling of atherosclerotic coronary arteries varies in relation to location and composition of plaque. Am J Cardiol 1999;84:135-140.

30. Fuji K, Carlier SG, Mintz GS, et al. Association of plaque characterization by intravascular ultrasound virtual histology and arterial remodeling. Am J Cardiol 2005;96:1476-1483. 


\title{
Chapter 9
}

\section{Dedicated Calibration Formulas Permit Correction of Differences Between Measurements by Different IVUS Devices as Demonstrated in Atherosclerotic Human Coronary Arteries in Vitro}

\author{
Marc Hartmann, Clemens von Birgelen, Gary S. Mintz, \\ Nadine Deppermann, Olaf Dirsch, Martin G. Stoel, \\ Gert K. van Houwelingen, Hans W. Louwerenburg, \\ Patrick M.J.Verhorst, Raimund Erbel
}

Reprinted with permission from:

The International Journal of Cardiovascular Imaging 2006;22(5):605-613.

(C) 2006 Springer Science+Business Media 



\section{Abstract}

Serial intravascular ultrasound (IVUS) measurements of coronary vessel dimensions are major endpoints of studies focusing on pharmacological interventions, efficiency of drug eluting stents, and vascular remodeling. In serial studies measurement variability among different IVUS devices may cause substantial misinterpretation and error.

We analyzed 33 human coronary plaques in vitro using two different IVUS systems (mechanical IVUS system with a $40 \mathrm{MHz}$ Atlantis SR catheter; solid-state electronic IVUS system with a $20 \mathrm{MHz}$ Invision catheter) and repeatedly measured the total vessel, lumen, and plaque+media cross-sectional area and plaque burden (plaque+media area divided by total vessel area). Between the "raw" measurements made by the two devices, there was a significant difference for both plaque+media area $\left(2.35 \pm 1.86 \mathrm{~mm}^{2}, P<0.01\right)$ and plaque burden $(5.39 \pm 3.68 \%$, $P<0.05)$. Measurements were then corrected by use of recently introduced calibration formulas; as a result the differences decreased significantly for all IVUS parameters measured $(P<0.0001)$. After correction, the remaining differences between the corrected mechanical and solid-state IVUS measurements similar to differences between repeated measurements with the same IVUS device (i.e., the intraobserver variability).

Thus, in serial studies the use of different IVUS devices at index and follow-up procedure may introduce a substantial error as a result of system-related differences. The application of dedicated calibration formulas allows for correction for these differences by decreasing such differences to the level of intraobserver variability.

\section{Introduction}

Quantitative assessment of coronary atherosclerosis during its natural history and following therapeutic interventions is important, as cardiovascular disease remains the most significant cause of death in western lifestyle nations. ${ }^{1}$ Intravascular ultrasound (IVUS) provides precise and highly reproducible measurements of coronary vessel dimensions ${ }^{2-7}$; it is increasingly used as a major endpoint in serial pharmacological progression- regression trials ${ }^{8-12}$, drug eluting stent trials ${ }^{13}$, and studies of coronary vascular remodeling. ${ }^{14,15}$ Despite the high reproducibility of measurements by an individual IVUS system ${ }^{6,7}$, there is a substantial variability of measurements obtained from different IVUS devices. ${ }^{16-22}$ Based on a study in phantoms, Schoenhagen et al. recently developed dedicated formulas for accurate post-hoc calibration to minimize these system-related differences. ${ }^{16}$ In the present manuscript we evaluated the impact of measurements with different IVUS devices (mechanical and solid-state electronic) in 33 human coronary segments in vitro and validated the calibration formulas by comparing the "corrected" differences versus the intraobserver variability of repeated measurements using the same system. 


\section{Methods}

Coronary vessel specimens

Thirteen coronary arteries (5 right coronary, 4 left anterior descending, 2 left circumflex, and 2 left main stems) were postmortem explanted from 7 patients (3 men, 4 women, age $74 \pm 6$ years). From these, a total of 33 coronary segments with $\leq 1$ major side branch were examined with IVUS. The procedure of this investigation was approved by the Local Council on Human Research.

\section{IVUS systems}

IVUS studies were performed with commercially available IVUS systems: a mechanical sector scanner in combination with a $40 \mathrm{MHz}$ single element beveled transducer (40 MHz Atlantis SR, Boston Scientific Corporation, Fremont, CA) or a solid-state device in combination with a phased-array catheter $(20 \mathrm{MHz}$ Invision, Volcano, Rancho Cordova, CA).

\section{IVUS imaging}

Major side branches of the coronary specimens were ligated and the proximal and distal ends were connected to sheaths fixed in a waterbath at a temperature of $37^{\circ} \mathrm{C}$. The arteries were continuously pressurized at $100 \mathrm{mmHg}$ by a $0.9 \% \mathrm{NaCl}$ infusion connected to the sidearm of the proximal sheath (Figure 1). ${ }^{23,24}$ During an initial manual IVUS interrogation, we identified a target atherosclerotic lesion in each segment, defined as the site with the (visually determined) greatest plaque burden. To facilitate matching of the target in different IVUS runs, we marked all target sites with needles in the adventitia. ${ }^{23} \mathrm{~A}$ total of 33 target lesions were identified. Then using both the solid-state and mechanical IVUS systems, we performed two continuous motorized pullbacks (two pullbacks for each IVUS system) from distal to proximal through each specimen using commercially available motorized pullback devices at a speed of $0.5 \mathrm{~mm} / \mathrm{s}$. The IVUS runs of the entire pullback were recorded on 0.5 -in high-resolution s-VHS tape for off-line analysis. 


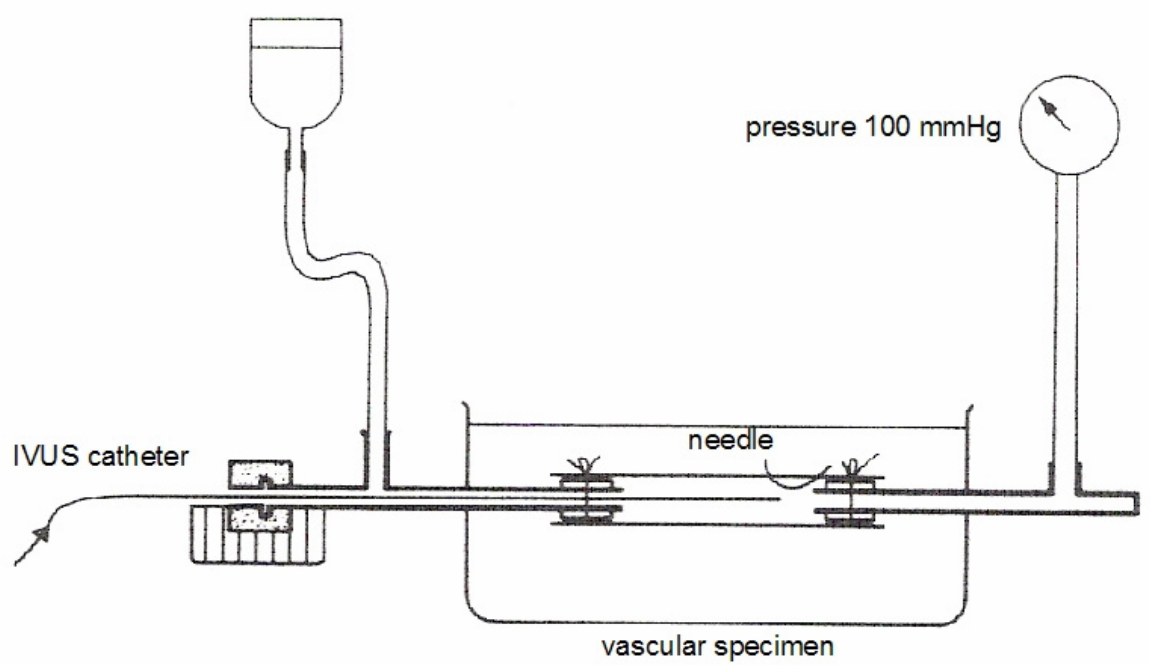

Figure 1. Setup of in vitro examination. Coronary arterial segments were connected to sheaths fixed in a waterbath and continuously pressurized. Target lesions identified, during IVUS interrogation, were marked with a needle in the adventitia.

\section{IVUS image analysis}

The pullbacks were then analyzed off-line on the corresponding acquisition machine of each IVUS system by a single experienced analyst. Exact matching of target site between the pullbacks was ensured using side-by-side comparison of the IVUS video sequences along with information of the pullback speed, characteristic calcifications, vascular and perivascular landmarks, the marker needle in the adventitia, and characteristics plaque shapes. In fact, this approach was very similar to the matching procedure as applied in previous IVUS studies in vitro and serial IVUS studies in vivo. ${ }^{8-12,14,15}$ For each IVUS pullback measurements were performed at each target lesion site. The lumen area was measured by tracing the leading edge of the intima. The total vessel area was measured by tracing the leading edge of the adventitia. As in previous IVUS studies plaque+media area (total vessel area minus lumen area) was a measure of atherosclerotic plaque because IVUS cannot measure media thickness accurately. ${ }^{2}$ The plaque burden (\%) was calculated as: (plaque+media area divided by total vessel area) $\times 100$. 


\section{Area calibration formulas}

All cross-sectional area measurements were then corrected by dedicated calibration formulas, as previously described in detail. ${ }^{16}$ For the $40 \mathrm{MHz}$ mechanical catheter the formula was: area after calibration $=2.309582-0.481960$ $x a+0.291338 \times a^{2}-0.024636 \times a^{3}+0.000923 \times a^{4}-0.000013 \times a^{5}$. For the 20 $\mathrm{MHz}$ solid-state catheter it was: area after calibration $=1.8585447-0.348552 \times \mathrm{a}+$ $0.334208 \times a^{2}-0.032221 \times a^{3}+0.001346 \times a^{4}-0.000020 \times a^{5}$.

Data analysis

First, we compared the "raw" measurements obtained with the 2 different IVUS systems (uncorrected-or "raw"-data from first pullback with solid-state device versus those from first pullback with mechanical device, respectively). Then calibration formulas were applied, and the comparison was repeated (corrected data from the first pullback with solid-state device versus corrected data from the first pullback with mechanical device, respectively). Finally, for each IVUS system separately, we compared measurements obtained from repeated pullbacks (corrected data from first versus second pullback).

\section{Statistical analysis}

Analyses were performed with SPSS 10.0.7 (SPSS Inc. Chicago, IL, USA). Quantitative data are presented as mean $\pm 1 S D$ and compared using student's t-test, analysis of variances, and linear regression analysis. According to Bland and Altman, the agreement between two measurements were assessed by determining the mean $\pm S D$ of the between measurement differences. ${ }^{25} \mathrm{~A} P$-value $<0.05$ was considered significant.

\section{Results}

Measurements with different IVUS catheters before calibration ("raw" measurements)

Table 1 presents differences between the "raw" measurements with the two IVUS devices at 33 target sites. There was a significant difference for plaque+media area $\left(2.35 \pm 1.86 \mathrm{~mm}^{2}, P<0.01\right)$ and plaque burden $(5.39 \pm 3.68 \%, P<0.05)$. On average, the solid-state device measured the areas to be smaller than the mechanical device (Table 1). 
Measurements with different IVUS catheters after calibration

After calibration, cross-sectional area measurements by the mechanical IVUS system decreased and measurements by the solid-state electronic IVUS system increased (Table 1). As a result of the application of the calibration formulas, differences between the 2 IVUS devices decreased significantly $(P<0.0001$ for all area measurements) (Table 1, Figure 2).

Table 1. IVUS measurements with different IVUS devices before and after correction by dedicated calibration formulas.

\begin{tabular}{lcccc}
\hline & $\begin{array}{c}\text { Mechanical } \\
\text { device }\end{array}$ & $\begin{array}{c}\text { Solid-state } \\
\text { device }\end{array}$ & Difference & $P$ \\
Before calibration $(\mathrm{n}=33)$ & & & & \\
Total vessel area $\left(\mathrm{mm}^{2}\right)$ & $16.2 \pm 6.7$ & $13.5 \pm 4.8$ & $2.7 \pm 2.2$ & 0.06 \\
Lumen area $\left(\mathrm{mm}^{2}\right)$ & $6.7 \pm 3.1$ & $6.3 \pm 2.7$ & $0.4 \pm 0.5$ & 0.6 \\
Plaque+media $a r e a\left(\mathrm{~mm}^{2}\right)$ & $9.6 \pm 4.2$ & $7.2 \pm 2.9$ & $2.4 \pm 1.9$ & $<0.01$ \\
Plaque burden $(\%)$ & $58.8 \pm 9.2$ & $53.4 \pm 10.2$ & $5.4 \pm 3.7$ & $<0.05$ \\
& & & & \\
After calibration $(\mathrm{n}=33)$ & $14.6 \pm 5.1$ & $14.6 \pm 5.2$ & $-0.0 \pm 0.5$ & 1.0 \\
Total vessel $a r e a\left(\mathrm{~mm}^{2}\right)$ & $6.5 \pm 3.1$ & $6.7 \pm 3.1$ & $-0.3 \pm 0.3$ & 0.7 \\
Lumen area $\left(\mathrm{mm}^{2}\right)$ & $8.1 \pm 2.9$ & $7.9 \pm 3.0$ & $0.3 \pm 0.5$ & 0.7 \\
Plaque+media $\mathrm{area}^{2}\left(\mathrm{~mm}^{2}\right)$ & $56.5 \pm 9.9$ & $54.2 \pm 10.2$ & $2.3 \pm 2.3$ & 0.4 \\
\hline Plaque burden $(\%)$ & & & & \\
\hline
\end{tabular}

Repeated measurements with same IVUS catheters

For all parameters measured, the differences between repeated measurements with the same IVUS device were similar for both systems (intraobserver variability; Table 2). In fact, the differences between repeated measurements with the same IVUS device were similar to the differences between measurements with two different IVUS devices after calibration (Figure 2). 


\section{Differences of area measurements $\left(\mathrm{mm}^{2}\right)$}

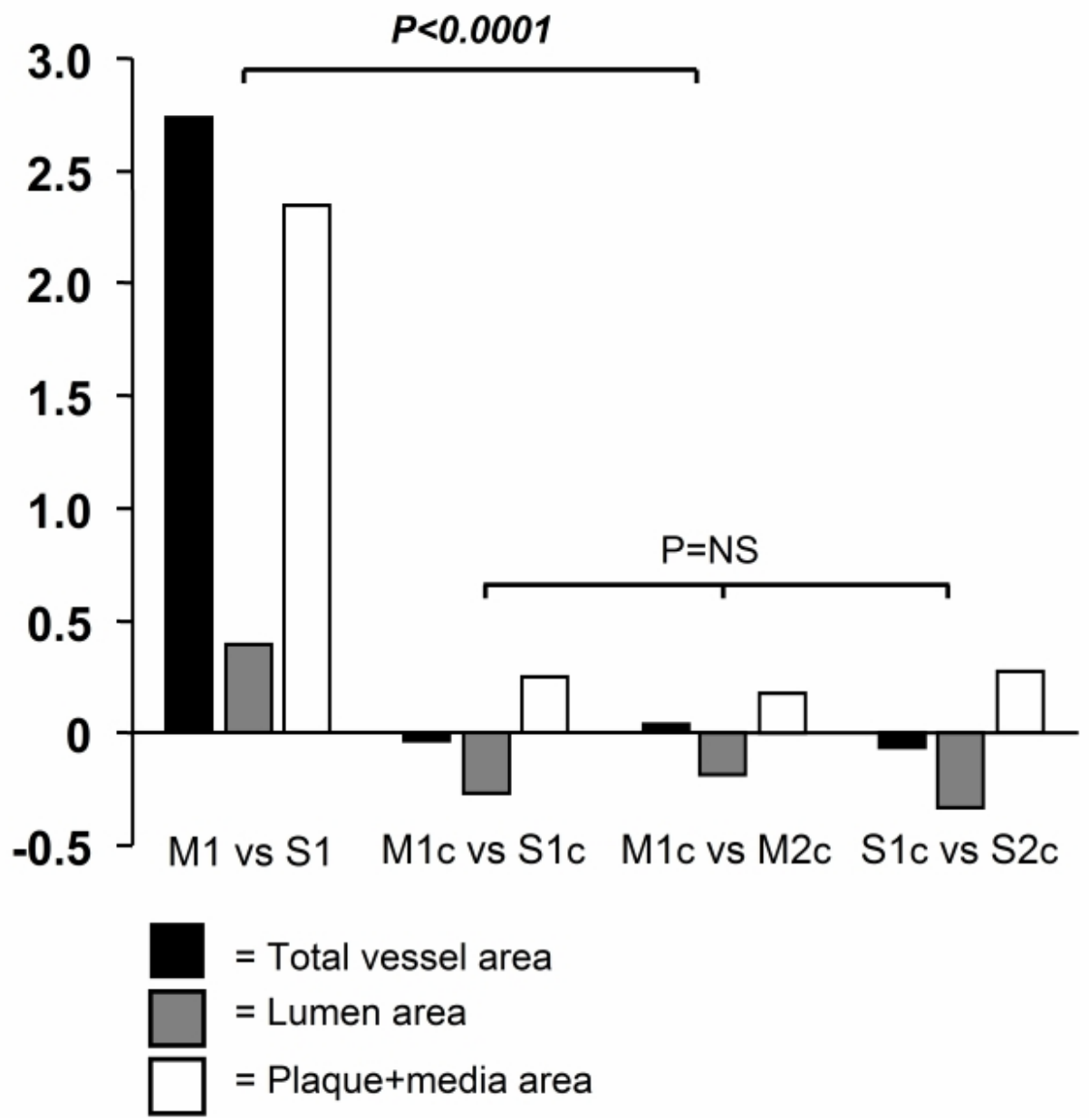

Figure 2. Effect of calibration on differences between both, area measurements by different IVUS devices and repeated measurements by the same device. Differences between the mechanical (M) and solid-state (S) device before correction (M1 vs. S1) decreased significantly after the correction (M1c vs. S1c). After calibration, differences between mechanical and solid-state device (M1c vs. S1c) did not differ from differences between repeated measurements with the same IVUS devices (M1c vs. M2c, and S1c vs. S2c), i.e. they were similar to the intraobserver variability of repeated IVUS measurements. $M 1(M 2)=$ first (second) measurement by the mechanical IVUS device; $S 1$ (S2) = first (second) measurement with the solid-state IVUS device; $c=$ corrected. 
Table 2. Repeated IVUS measurements with the same IVUS device.

Data represent measurements corrected by the dedicated calibration formulas.

\begin{tabular}{lcccc}
\hline & $\mathbf{1}^{\text {st }}$ Pullback & $\mathbf{2}^{\text {nd }}$ Pullback & Difference & P \\
Mechanical device $(\mathrm{n}=33)$ & & & & \\
& & & & \\
Total vessel area $\left(\mathrm{mm}^{2}\right)$ & $14.6 \pm 5.1$ & $14.6 \pm 5.1$ & $0.0 \pm 0.2$ & 1.0 \\
Lumen area $\left(\mathrm{mm}^{2}\right)$ & $6.5 \pm 3.1$ & $6.6 \pm 3.1$ & $-0.2 \pm 0.4$ & 0.9 \\
Plaque+media area $\left(\mathrm{mm}^{2}\right)$ & $8.1 \pm 2.9$ & $8.1 \pm 2.9$ & $0.2 \pm 0.3$ & 0.9 \\
Plaque burden $(\%)$ & $56.5 \pm 9.9$ & $55.7 \pm 10.8$ & $0.8 \pm 3.1$ & 0.8 \\
& & & & \\
Solid-state device $(\mathrm{n}=33)$ & & & & \\
Total vessel area $\left(\mathrm{mm}^{2}\right)$ & $14.6 \pm 5.2$ & $14.7 \pm 5.1$ & $-0.1 \pm 0.5$ & 1.0 \\
Lumen area $\left(\mathrm{mm}^{2}\right)$ & $6.7 \pm 3.1$ & $7.1 \pm 3.3$ & $-0.3 \pm 0.5$ & 0.7 \\
Plaque+media $a r e a\left(\mathrm{~mm}^{2}\right)$ & $7.9 \pm 3.0$ & $7.6 \pm 2.6$ & $0.3 \pm 0.8$ & 0.7 \\
Plaque burden $(\%)$ & $54.2 \pm 10.2$ & $53.0 \pm 9.9$ & $1.2 \pm 4.2$ & 0.6 \\
\hline
\end{tabular}

Regression analysis before and after calibration

Both, before and after calibration there was a significant positive linear relation between the cross-sectional area measurements by the two different IVUS devices $(\mathrm{r}=0.93-1.0, P<0.0001$, Figure 3$)$. However, importantly, before calibration the slope of the regression line was 0.70 for total vessel, 0.87 for lumen, and 0.93 for plaque+media cross-sectional area, while after calibration it was 1.0 for each of these parameters (Figure 3). Figure 4 shows differences between cross-sectional area measurements by the mechanical versus solid-state device plotted against the mean of 2 measurements, both for the uncorrected "raw" data and after correction with the dedicated calibration formulas. Of note, the greatest differences were observed in larger vessels with a particularly great distance between transducer and target (Figure 4). 

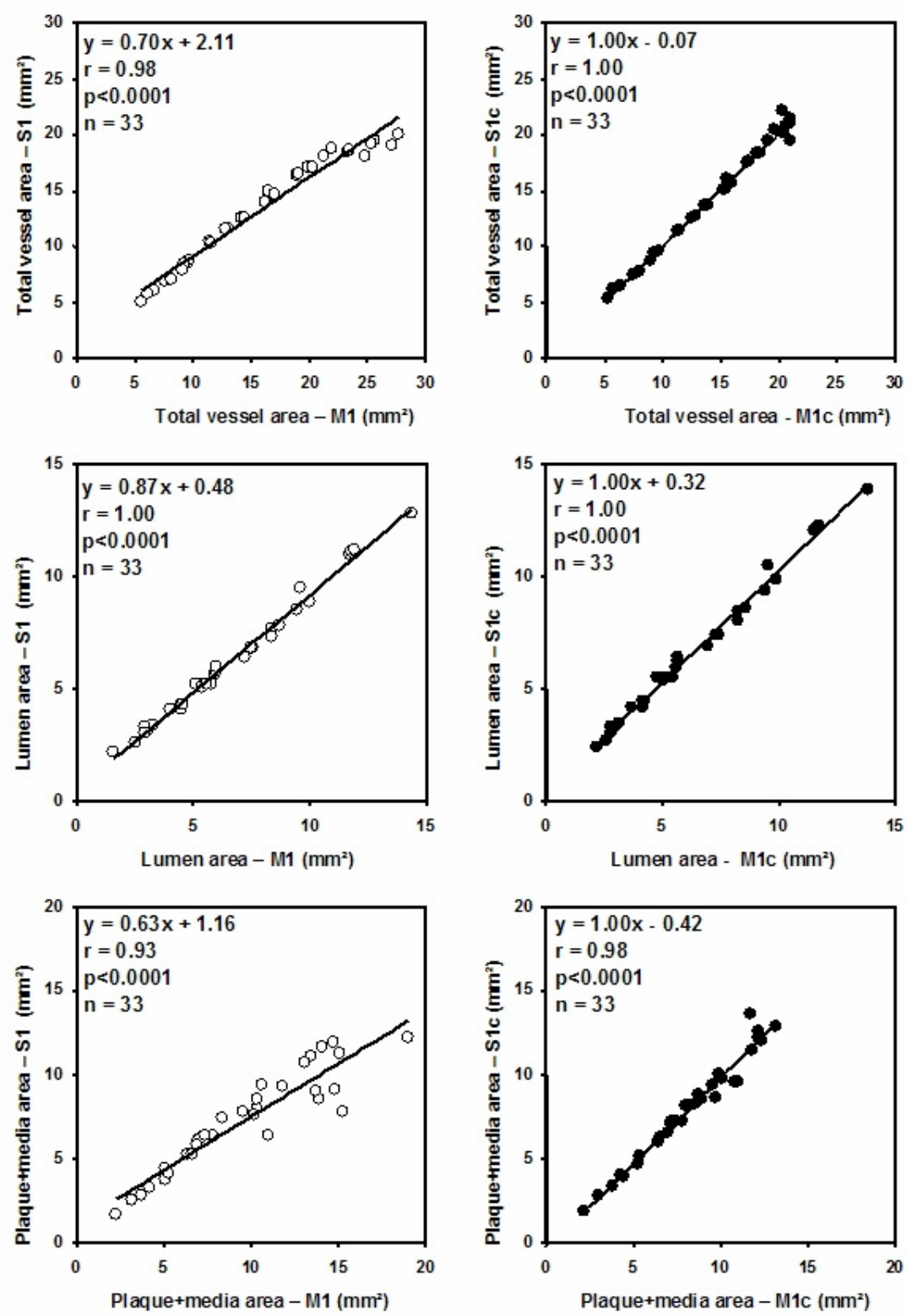

Figure 3. Cross-sectional area measurements by mechanical versus solid-state device. Before (left panels, white dots) and after correction with the calibration formulas (right panels, black dots), there was a high correlation between the area measurements by the two different IVUS devices. But importantly, the slope of the regression lines improved substantially after the correction. 

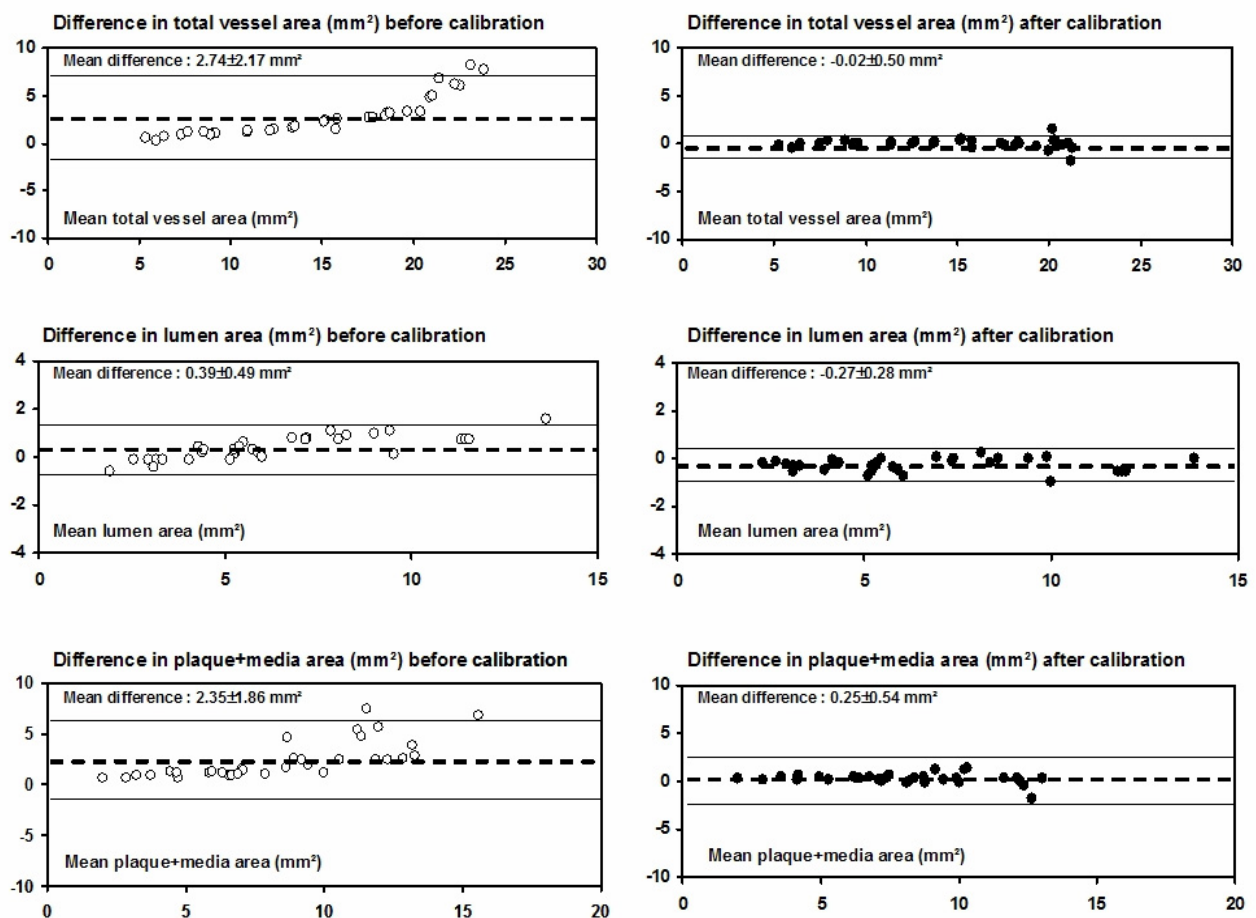

Difference in plaque+media a rea $\left(\mathrm{mm}^{2}\right)$ after calibration

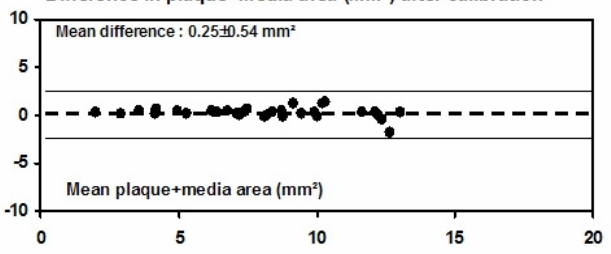

Figure 4. Differences between area measurements by mechanical and solid-state IVUS device versus the mean of these two measurements. Data before (left panels, white dots) and after correction with the calibration formulas (right panels, black dots) are presented, showing clearly the effect of the calibration. In each panel, the dotted line and the two continuous lines indicate the mean \pm 2 standard deviations of the difference.

\section{Discussion}

This IVUS study in atherosclerotic human coronary arteries demonstrated a significant difference between repeated cross-sectional IVUS measurements with a mechanical versus solid state IVUS device as compared to repeated measurements with the same device (from two IVUS pullbacks). Serial studies when different IVUS devices are used at index and follow-up may introduce a substantial error as a result of such system-related differences. Our study also confirms that dedicated calibration formulas, as developed by Schoenhagen et al. based on his work in cylindric acrylic phantoms ${ }^{16}$, permit reliable correction for such differences in atherosclerotic human coronary arteries. After correction with the formulas, the difference between measurements with the mechanical and the solid 
state IVUS system decreased significantly. We were able to show that after correction with the calibration formulas, the remaining variability between measurements made by using two different IVUS systems was comparable to the measurement variability from repeated pullbacks of the same IVUS device. That is, correction with the calibration formulas decreased the measurement difference to the level of the intraobserver variability (total vessel area $0.1 \pm 0.5 \mathrm{~mm}^{2}$; lumen area $0.3 \pm 0.5 \mathrm{~mm}^{2}$; plaque+media area $0.3 \pm 0.8 \mathrm{~mm}^{2}$ ).

Our data in atherosclerotic coronary specimens corroborate previous studies that indicated discrepancies between repeated measurements with different IVUS catheters and/or systems in tubular phantoms $s^{16,18,20,21}$ and in vivo. ${ }^{17,18,22}$ In addition, studies have previously addressed the variability of different IVUS machines with regards to the visualization of coronary wall structures in vitro ${ }^{19}$ and quantitative tissue characterization in vivo. ${ }^{22}$ Based on their work in cylindrical phantoms, Bruining et al. described a helpful calibration formula for the adjustment of measurements performed with mechanical $30 \mathrm{MHz}$ IVUS catheters connected to a certain IVUS console. $^{20}$

\section{Implications for serial IVUS studies}

Our data suggest that the results of serial IVUS studies can be affected by the use of different IVUS systems if no correction is performed. The use of dedicated calibration formulas, however, permits a reliable correction. Awareness of these circumstances is particularly important as various serial IVUS studies address relatively small changes that might disappear if different IVUS systems are used without correction. Evidence for this trend can easily be supplied by a few examples: (1) Serial changes of the coronary plaque dimensions as assessed with IVUS are major endpoints in pharmacological intervention trials. ${ }^{8-12}$ Plaque changes may often be small, but plaque progression with IVUS has been shown to reflect cardiovascular risks. ${ }^{26}$ (2) Serial IVUS assessment of the total vessel area is the "gold standard" for the serial analysis of coronary artery remodeling. ${ }^{2,14,15}$ In the present study, the difference between the (uncorrected) measurements of the total vessel area as measured with the two different IVUS systems was similar to annual changes in total vessel area. ${ }^{14}$ (3) Serial IVUS examinations in the context of coronary stenting currently address relatively small changes in neointimal volume, because the extent of intimal hyperplasia has decreased significantly with the introduction of drug eluting stents. ${ }^{13}$

Serial IVUS trials should preferably use a single IVUS device to avoid the introduction of this undesirable, systematic error. If clinical trials are not restricted to a single IVUS device, in each individual patient the same device should be used 
for index and follow-up analysis. Application of dedicated calibration formulas may then be performed to reliably correct for discrepancies between different IVUS catheters and/or systems. ${ }^{16,20}$ If volumetric IVUS parameters are calculated from the cross-sectional IVUS data based on Simpson's rule, the errors of crosssectional area measurements with different IVUS systems will translate into an error of the volumetric data. ${ }^{16,27}$ This is particularly important as plaque volume is currently a common target of trials that assess the pharmacological therapy of coronary plaque progression. ${ }^{1,7,10-12}$ To prevent such error, we suggest to correct the cross-sectional area measurements before the volumetric data are derived. This approach increases the reliability of IVUS measurements and facilitates the comparison of data obtained with different technical setups in various IVUS studies.

\section{Limitations}

As in previous IVUS studies in coronary specimen in vitro ${ }^{3-5,19,23,24}$, the sample size was limited. The experimental setup differs from in vivo imaging (e.g., flushed with water instead of blood and a constant perfusion pressure instead of a cyclic pressure). ${ }^{16,23,24}$ Nevertheless, in the present study we studied diseased coronary vessels with a state of atherosclerosis that may be comparable to the disease that is examined in IVUS progression-regression studies. In the in vitro setting the marker needle and the centered position of the IVUS transducer ${ }^{28}$ avoided some artifacts and optimized both imaging and matching of the target site during repeated IVUS pullbacks. We did not perform a histomorphometric analysis.

\section{Conclusion}

In serial studies the use of different (mechanical versus solid-state) IVUS devices at index and follow-up may introduce a substantial error as a result of systemrelated differences. Our data in atherosclerotic coronary arteries in vitro show that the application of dedicated calibration formulas allows for correction for these differences and decreases them to the level of intraobserver variability.

\section{References}

1. Schoenhagen P, Nissen SE. Coronary atherosclerotic disease burden: an emerging endpoint in progression/regression studies using intravascular ultrasound. Curr Drug Targets Cardiovasc Haematol Disord 2003;3:218-226.

2. Mintz GS, Nissen SE, Anderson WD, et al. American College of Cardiology clinical expert consensus document on standards for acquisition, measurement and reporting of intravascular ultrasound studies (IVUS). J Am Coll Cardiol 2001;37:1478-1492. 
3. Potkin BN, Bartorelli AL, Gessert JM, et al. Coronary artery imaging with intravascular highfrequency ultrasound. Circulation 1992;68:276-281.

4. Nishimura RA, Edwards WD, Warnes CA, et al. Intravascular ultrasound imaging: in vitro validation and pathologic correlation. J Am Coll Cardiol 1990;16:145-154.

5. Bartorelli AL, Neville RF, Keren G, et al. In vitro and in - vivo intravascular ultrasound. Eur Heart J 1992;13:102-108.

6. Foster GP, Mittleman MA, Koch M, et al. Variability in the measurement of intracoronary ultrasound images: implications for the identification of atherosclerotic plaque regression. Clin Cardiol 1997;20:11-15.

7. Bocksch W, Wellnhofer E, Schartl M, et al. Reproducibility of serial intravascular ultrasound measurements in patients with angiographically silent coronary artery disease after heart transplantation. Coron Artery Dis 2000;11:555-562.

8. Takagi T, Yoshida K, Akasaka T, et al. Intravascular ultrasound analysis of reduction in progression of coronary narrowing by treatment with pravastatin. Am J Cardiol 1997;79:1673-1676.

9. von Birgelen C, Hartmann M, Mintz GS, et al. Relation between progression and regression of atherosclerotic left main coronary artery disease and serum cholesterol levels as assessed with serial long-term ( $\geq 12$ months) follow-up intravascular ultrasound. Circulation 2003;108:2757-2762.

10. Jensen LO, Thayssen P, Pederson KE, et al. Regression of coronary atherosclerosis by simvastatin: a serial intravascular ultrasound study. Circulation 2004;110:265-270.

11. Nissen SE, Tuzcu EM, Schoenhagen $P$, et al. Effect of intensive compared with moderate lipidlowering therapy on progression of coronary atherosclerosis. A randomized controlled trial. JAMA 2004;291:1071-1080.

12. Nissen SE, Tsunoda T, Tuzcu EM, et al. Effect of recombinat ApoA-I Milano on coronary atherosclerosis in patients with acute coronary syndrome: a randomized controlled trial. JAMA 2003;290:2292-2300.

13. Serruys PW, Degertekin M, Tanabe K, et al. Intravascular ultrasound findings in the multicenter, randomized, double-blind RAVEL (Randomized study with sirolimus- eluting Velocity balloonexpandable stent in the treatment of patients with de novo native coronary artery Lesions) trial. Circulation 2002;106:798-803.

14. von Birgelen C, Hartmann M, Mintz GS, et al. Spectrum of remodeling behavior observed with serial long-term ( $\geq 12$ months) follow-up intravascular ultrasound studies in left main coronary arteries. Am J Cardiol 2004;93:1107-1113.

15. Shiran A, Mintz GS, Leiboff B, et al. Serial volumetric intravascular ultrasound assessment of arterial remodeling in left main coronary artery disease. Am J Cardiol 1999;83:1427-1432.

16. Schoenhagen P, Shelly KS, Tuzcu E, et al. Variability of area measurements obtained with different intravascular ultrasound catheter systems: impact on clinical trials and a method for accurate calibration. J Am Soc Echocardogr 2003;16:277-284. [Erratum: J Am Soc Echocardiogr 2005;18:193].

17. Tardif JC, Bertrand OF, Mongrain R, et al. Reliability of mechanical and phased-array designs for serial intravascular ultrasound examinations-animal and clinical studies in stented and non-stented coronary arteries. Int J Card Imaging 2000;16:365-375.

18. Li Y, Honye J, Saito $\mathrm{S}$, et al. Variability in quantitative measurement of the same segment with two different intravascular ultrasound systems: in vivo and in vitro studies. Catheter Cardiovasc Interv 2004;62:175-180.

19. Hiro $\mathrm{T}$, Leung $\mathrm{CY}$, Russo RJ, et al. Variability of a three-layerd appearance in intravascular ultrasound coronary images: a comparison of morphometric measurements with four intravascular ultrasound systems. Am J Card Imaging 1996;10:219-227.

20. Bruining N, Hamers R, Teo TJ, et al. Adjustment method for mechanical Boston scientific corporation $30 \mathrm{MHz}$ intravascular ultrasound catheters connected to a clearview console. Mechanical 30 MHz IVUS catheter adjustment. Int J Cardiovasc Imaging 2004;20:83-91. 
21. Staehr P, Rupprecht HJ, Voigtlander $\mathrm{T}$, et al. Importance of calibration for diameter and area determination by intravascular ultrasound. Int J Card Imaging 1996;12:221-229.

22. Rodriguez-Granillo GA, Fadden EP, Aoki J, et al. In vivo variability in quantitative coronary ultrasound and tissue characterization measurements with mechanical and phased-array catheters. Int J Cardiovasc Imaging 2006;22:47-53.

23. von Birgelen C, van der Lugt A, Nicosia A, et al. Computerized assessment of coronary lumen and atherosclerotic plaque dimensions in three-dimensional intravascular ultrasound correlated with histomorphometry. Am J Cardiol 1996;78:1202-1209.

24. Palmer ND, Northridge D, Lessells A, et al. In vitro analysis of coronary atheromatous lesions by intravascular ultrasound. Eur Heart J 1999;20:1701-1706.

25. Bland JM, Altman DG. Statistical methods for assessing agreement between two methods of clinical measurement. Lancet 1986;2:307-310.

26. von Birgelen C, Hartmann M, Mintz GS, et al. Relationship between cardiovascular risk as predicted by established risk scores versus plaque progression as measured by serial intravascular ultrasound. Circulation 2004;110:1579-1585.

27. von Birgelen C, de Feyter PJ, de Vrey EA, et al. Simpson's rule for the volumetric ultrasound assessment of atherosclerotic coronary arteries: a study with ECG-gated threedimensional intravascular ultrasound. Coron Artery Dis 1997;8:363-369.

28. Bekeredjian R, Hardt $S$, Just $A$, et al. Influence of catheter position and equipmentrelated factors on the accuracy of intravascular ultrasound measurements. J Invasiv Cardiol 1999;11:207-212. 



\section{Chapter 10}

\section{Reproducibility of Volumetric Intravascular Ultrasound Radiofrequency-based Analysis of Coronary Plaque Composition in Vivo}

Marc Hartmann, Eline S.K. Mattern, Jennifer Huisman, Gert K. van Houwelingen, Frits H.A.F. de Man, Martin G. Stoel, Peter W. Danse, Hans W. Louwerenburg, Clemens von Birgelen

Reprinted with permission from:

The International Journal of Cardiovascular Imaging 2008; - in press DOI: 10.1007/s10554-008-9338-9

(C) 2008 Springer Science+Business Media 



\section{Abstract}

Intravascular ultrasound radiofrequency (RF-IVUS) data permit the analysis of coronary plaque composition in vivo and is used as an endpoint of ongoing pharmacological intervention trials.

We assessed the reproducibility of volumetric RF-IVUS analyses in mild-to-moderately diseased atherosclerotic human coronary arteries in vivo. A total of 9,212 IVUS analyses on cross-sectional IVUS frames was performed to evaluate the reproducibility of volumetric RF-IVUS measurements in 33 coronary segments with a length of $27 \pm 7 \mathrm{~mm}$. For vessel, lumen, and plaque+media volume the relative measurement differences ( $P=\mathrm{NS}$ for all) were ( $\mathrm{A}=$ intraobserver comparison, same pullback) $-0.40 \pm 1.0 \% ;-0.48 \pm 1.4 \% ;-0.35 \pm 1.6 \%$, ( $B=$ intraobserver comparison, repeated pullback) $-0.42 \pm 1.2 \%$; $-0.52 \pm 1.8 \% ;-0.43 \pm 4.5 \%$ ( $C=$ interobserver comparison, same pullback) $0.71 \pm 1.8 \%$; $0.71 \pm 2.2 \%$, and $0.89 \pm 5.0 \%$, respectively. For fibrous, fibro-lipidic, calcium, and necrotic-core volumes the relative measurement differences ( $P=\mathrm{NS}$ for all) were (A) $0.45 \pm 2.1 \% ;-1.12 \pm 4.9 \% ;-0.84 \pm 2.1 \% ; \quad-0.22- \pm 1.8 \%$, (B) $1.40 \pm 4.1 \% ; 1.26 \pm 6.7 \% ; 2.66 \pm 7.4 \%$; $0.85 \pm 4.4 \%$, and $(C)-1.60 \pm 4.9 \% ; 3.85 \pm 8.2 \% ; 1.66 \pm 7.5 \%$, and $-1.58 \pm 4.7 \%$, respectively. Of note, necrotic-core volume showed on average the lowest measurement variability.

Thus, in mild-to-moderate atherosclerotic coronary artery disease the reproducibility of volumetric compositional RF-IVUS measurements from the same pullback is relatively high, but lower than the reproducibility of geometrical IVUS measurements. Measurements from repeated pullbacks and by different observers show acceptable reproducibilities; the volumetric measurement of the necrotic-core shows on average the highest reproducibility of the compositional RF-IVUS measurements.

\section{Introduction}

Both, the progression of atherosclerotic plaque volume and unfavorable plaque tissue characteristics contribute to the overall risk of cardiovascular events. ${ }^{1-5}$ Previous progression-regression studies with intravascular ultrasound (IVUS) reported a certain beneficial effect of anti-atherosclerotic pharmacological therapies on the progression of plaque volume; however, this can only partly explain the clinical benefit observed. ${ }^{6-10}$

While conventional grey-scale IVUS permits accurate quantification of plaque and vessel dimensions, it has significant limitations in the assessment of plaque composition. ${ }^{6,711-15}$ As a consequence, a spectral analysis of IVUS radiofrequency (RF-IVUS) data has been developed and is increasingly used in clinical research. ${ }^{16-}$ ${ }^{22}$ RF-IVUS quantifies coronary plaque components (e.g., the necrotic core) with a high predictive accuracy, as demonstrated in vitro and in vivo. ${ }^{23-25}$ Volumetric RFIVUS data may be interesting endpoints of IVUS studies that evaluate antiatherosclerotic pharmacological therapies. ${ }^{26}$ However, there is a lack of information on the reproducibility of this approach. ${ }^{17}$

Therefore, we assessed in atherosclerotic human coronary segments the reproducibility of volumetric RF-IVUS in vivo by comparing analyses from the same pullback, repeated pullbacks, and by different observers. 


\section{Methods}

\section{Study population}

To assess the reproducibility of segmental IVUS-RF data analysis in mild-tomoderately diseased atherosclerotic human coronary arteries in vivo, we repeatedly analyzed IVUS data sets of 33 at least $20 \mathrm{~mm}$-long coronary segments from non-target and non-treated coronary arteries with an angiographic lumen diameter stenosis $<50 \%$ and absence of severe calcification or vessel tortuosity. Two motorized IVUS pullbacks at a speed of $0.5 \mathrm{~mm} / \mathrm{s}$ were available from 16 patients in regular sinus rhythm in whom clinically-driven, elective diagnostic cardiac catheterizations $(n=10)$ or percutaneous coronary interventions $(n=6)$ required IVUS assessment. Informed consent was obtained prior to cardiac catheterization from all patients in accordance with the directives of the Local Medical Ethics Committee.

\section{RF-IVUS}

Details regarding the RF-IVUS technique, also known as Virtual Histology IVUS, and validation have previously been described..$^{16,17,23-25}$ In brief, spectral analysis of IVUS radiofrequency data is used to create tissue maps in order to classify atherosclerotic plaques into four major components (fibrous, fibro-lipidic, calcium, and necrotic-core). Different plaque components were represented according to a dedicated color code (green, light-green, white and red) (Figure 1).

\section{RF-IVUS acquisition}

RF-IVUS data was acquired with commercially available phased-array IVUS catheters (Eagle Eye Gold 2.9F $20 \mathrm{MHz}$, Volcano Corporation, Rancho Cordova, USA) by a dedicated RF-IVUS console (Volcano Therapeutics, Rancho Cordova, USA). The image acquisition was ECG-gated (Figure 1). The catheter probe was advanced $\geq 10 \mathrm{~mm}$ distal to the most distal side-branch. Angiographic cine runs were performed to define the position of the IVUS catheter. After intracoronary injection of $200 \mu \mathrm{g}$ nitroglycerine, a continuous pullback of the IVUS catheter was perfomed using a motorized pullback device at $0.5 \mathrm{~mm} / \mathrm{s}$ (TrackBackll, Volcano Corporation, Rancho Cordova, USA). In the patients of the present study, a second pullback was performed using the same IVUS catheter. Data of both pullbacks were stored on hard disk. 
RF-IVUS analysis

RF-IVUS analysis was performed off-line by two experienced IVUS analysts (M.H., E.S.K.M.). The first pullback (I) was divided into segments as the region of interest. Segments were located between two adjacent side-branches and contained no major calcification that could have limited quantitative assessment of vessel crosssectional area. Subsequently, the same segment was identified in the second pullback (II) using side-by-side comparison of longitudinal and cross-sectional views. In fact, this approach was similar to the matching procedure as applied in serial IVUS studies. ${ }^{7-10}$ The time required for the matching procedure was recorded. The vessel borders were traced using a semi-automatic contour detection program (IVUSLab 4.4, Volcano Corporation, Rancho Cordova, USA).

The borders of each cross-sectional image per segment were manually corrected if required to meet a high standard of accuracy. The lumen border was detected by tracing the leading edge of the intima, and the total vessel border by tracing the leading edge of the adventitia. Plaque+media (total vessel - lumen) was used as a measure of atherosclerotic plaque. ${ }^{11,12}$ The time required for the correction of the computerized contour detection was recorded. For each coronary segment, the vessel geometrics (lumen, total vessel and plaque+media volume) and the volumetric compositional RF-IVUS data (fibrous, fibro-lipidic, calcium, and necrotic-core) were generated by applying Simpson's rule (Figure 1). ${ }^{22,27}$ Volumetric plaque burden (\%) was calculated as [(plaque volume/total vessel volume) $\times 100]$.

Each segment of the first pullback (I) was analyzed twice by the 1st observer (la and $\mathrm{Ib}$ ) and once by the 2nd observer (Ic) after re-defining the segments of the first pullback; the corresponding segment of the second pullback (II) was analyzed once by the 1 st observer.

Data analysis

First, we compared repeated RF-IVUS measurements (volumetric vessel geometrics and composition) from the first pullback analyzed by the 1st observer (intraobserver comparison, la versus Ib). Second, we compared the RF-IVUS measurements from the first and second pullback analyzed by the 1st observer (intraobserver comparison, la versus II). Third, we compared RF-IVUS measurements from the first pullback between the 1st and 2nd observer (interobserver comparison, la versus Ic). 
Statistical analysis

Analyses were performed with SPSS 13.0 (SPSS Inc., Chicago, Illinois). Dichotomous data are presented as frequencies. Quantitative data are presented as mean $\pm 1 S D$ and compared using student t-test, linear regression analysis, or ANOVA for repeated measures with post-hoc testing with Tukey honestly significant difference test. According to Bland and Altman, the agreement between two measurements were assessed by determining the mean $\pm 2 S D$ of the between measurement differences. ${ }^{28} \mathrm{~A}$ two-sided $\mathrm{p}$-value $<0.05$ was considered significant.

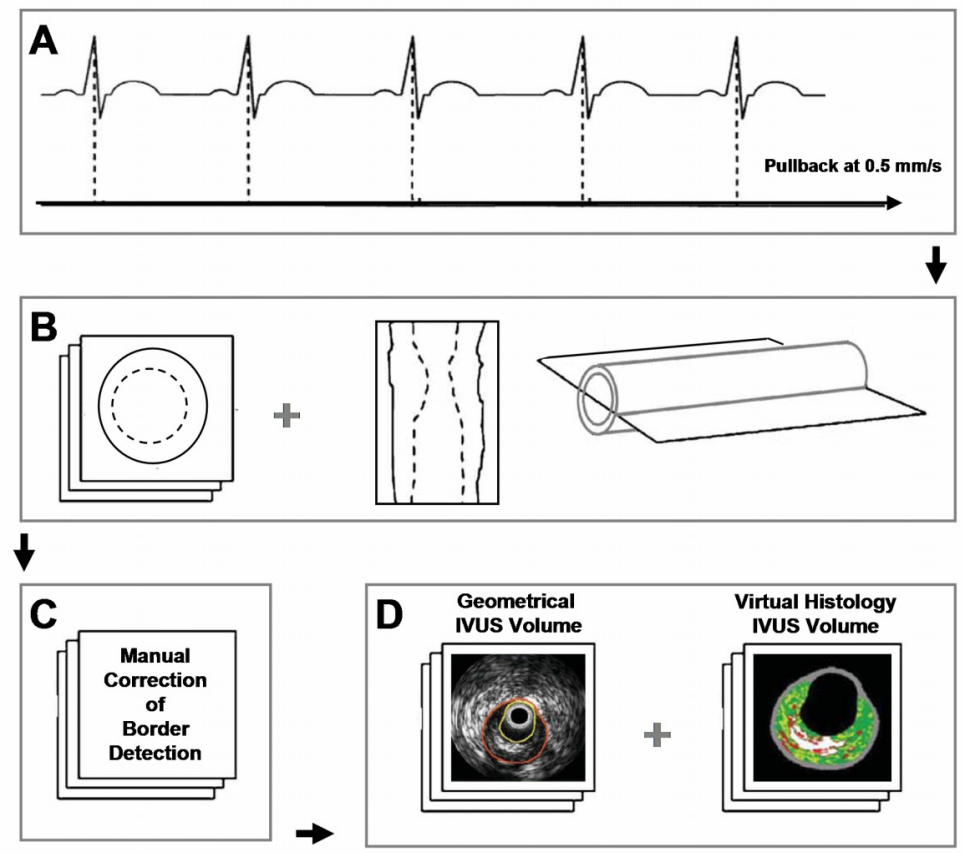

Figure 1. Image data acquisition and analysis. The R-wave peak triggered the ECGgated image aquisition during a motorized pullback at a speed of $0.5 \mathrm{~mm} / \mathrm{s}(\mathbf{A})$. Automated contour detection of the luminal and vessel borders was performed on all IVUS images acquired. A longitudinal reconstruction of the segment was obtained from the entire threedimensional "stack" of images (B). The direction of the longitudinal reconstruction was indicated by an arrowhead on the cross-sectional images. All contours were checked on the cross-sectional frame and manual correction could be performed (C). VH-IVUS analysis revealed (colour-encoded) information on plaque composition from the space between the lumen and vessel borders; volumetric data of the geometrical vessel dimension and of plaque composition were automatically generated for the segment (D). 


\section{Results}

\section{Study population}

Thirty-three atherosclerotic coronary segments (six proximal and six in the mid right, seven proximal and six in the mid left anterior descending, five proximal and three in the mid left circumflex coronary artery, respectively) from 16 patients with stable angina (11 men, age $58 \pm 8$ years) were included. The heart rate was $69 \pm 14 \mathrm{bpm}$. There were no complications related to the IVUS investigation.

\section{RF-IVUS analysis and data}

A total of 9,212 cross-sectional IVUS analyses were performed (70 \pm 21 frames/segment). Manual correction of the automatic border detection was required in $96 \pm 6 \%$ of frames/segment within $40 \pm 11 \mathrm{~min} /$ segment. Matching the segments of interest between the repeated pullbacks I and II required $13 \pm 5 \mathrm{~min} /$ segment. The length of the segments was $27 \pm 7 \mathrm{~mm}$ (range: $20-46 \mathrm{~mm}$ ) with a volumetric plaque burden of $36 \pm 8 \%$ (range: $23-55 \%$ ). The atherosclerotic segments contained pre-dominantly fibrous $(47 \%)$ and necrotic-core $(25 \%)$ tissue. Further data are presented in Table 1.

Table 1 (part 1). Volumetric RF-IVUS measurements of vessel geometrics and plaque composition of $1^{\text {st }}$ and $2^{\text {nd }}$ observer.

\begin{tabular}{|c|c|c|c|c|}
\hline & & $1^{\text {st }}$ Observer & & $2^{\text {nd }}$ Observer \\
\hline & Pullback la & Pullback lb & Pullback II & Pullback Ic \\
\hline \multicolumn{5}{|l|}{ Vessel Geometrics } \\
\hline Total Vessel Volume $\left(\mathrm{mm}^{3}\right)$ & $458.98 \pm 118.1$ & $460.24 \pm 116.3$ & $460.85 \pm 118.1$ & $456.27 \pm 119.6$ \\
\hline Lumen Volume $\left(\mathrm{mm}^{3}\right)$ & $290.05 \pm 77.7$ & $291.11 \pm 76.8$ & $291.33 \pm 77.5$ & $288.09 \pm 77.9$ \\
\hline Plaque+media Volume $\left(\mathrm{mm}^{3}\right)$ & $168.93 \pm 70.4$ & $169.12 \pm 69.0$ & $169.32 \pm 70.3$ & $168.17 \pm 72.3$ \\
\hline Plaque Burden(\%) & $36.36 \pm 8.3$ & $36.33 \pm 8.2$ & $36.31 \pm 8.2$ & $36.34 \pm 8.5$ \\
\hline
\end{tabular}


Table 1 (part 2). Volumetric RF-IVUS measurements of vessel geometrics and plaque composition of $1^{\text {st }}$ and $2^{\text {nd }}$ observer.

\begin{tabular}{|c|c|c|c|c|}
\hline & & $1^{\text {st }}$ Observer & & $\begin{array}{l}2^{\text {nd }} \\
\text { Observer }\end{array}$ \\
\hline & Pullback la & Pullback lb & Pullback II & Pullback Ic \\
\hline \multicolumn{5}{|l|}{ Plaque Composition } \\
\hline Fibrous Volume $\left(\mathrm{mm}^{3}\right)$ & $34.06 \pm 32.0$ & $34.10 \pm 32.0$ & $33.68 \pm 31.7$ & $34.35 \pm 32.1$ \\
\hline Fibrous (\%) & $46.62 \pm 15.5$ & $46.44 \pm 15.8$ & $46.64 \pm 15.8$ & $46.98 \pm 15.3$ \\
\hline Fibro-Lipidic Volume $\left(\mathrm{mm}^{3}\right)$ & $5.66 \pm 7.7$ & $5.70 \pm 7.9$ & $5.55 \pm 7.5$ & $5.49 \pm 7.2$ \\
\hline Fibro-Lipidic (\%) & $6.94 \pm 4.8$ & $6.82 \pm 4.7$ & $6.93 \pm 4.6$ & $6.67 \pm 4.8$ \\
\hline Calcium Volume $\left(\mathrm{mm}^{3}\right)$ & $12.46 \pm 9.5$ & $12.48 \pm 9.3$ & $12.36 \pm 9.5$ & $12.48 \pm 9.9$ \\
\hline Calcium (\%) & $21.85 \pm 11.7$ & $21.98 \pm 11.8$ & $21.73 \pm 11.8$ & $21.40 \pm 11.6$ \\
\hline Necrotic-Core Volume $\left(\mathrm{mm}^{3}\right)$ & $15.94 \pm 13.5$ & $16.00 \pm 13.5$ & $15.77 \pm 13.1$ & $16.15 \pm 13.3$ \\
\hline Necrotic-Core (\%) & $24.59 \pm 8.5$ & $24.62 \pm 8.7$ & $24.70 \pm 8.6$ & $24.83 \pm 8.7$ \\
\hline
\end{tabular}

Reproducibility of geometrical RF-IVUS (intraobserver and interobserver comparison)

Data on the agreement and variability of repeated geometrical RF-IVUS measurements (absolute and/or relative differences) showed a high reproducibility (Table 2 and Figure 2). Repeated geometrical measurements were highly correlated for intraobserver comparison from the same pullback and repeated pullbacks, and for the interobserver comparison ( $r \geq 0.999, \geq 0.997$, and $\geq 0.995$; $P<0.001$ for all; data not shown). The limits of agreement (i.e., 2SD of mean difference), which indicate the expected relative difference between 2 measurements (Figure 2), were higher for two observers who analyzed the same IVUS pullback than for the same observer who analyzed repeated pullbacks, with 
significantly lower relative differences for total vessel and lumen volume $(P<0.01$ for both, Table 2).

Table 2 (part 1). Intra- and interobserver measurement differences of volumetric vessel geometrics and plaque composition.

\begin{tabular}{|c|c|c|c|c|}
\hline & $\begin{array}{l}\text { Intraobserver } \\
\text { Comparsion } \\
\text { (same } \\
\text { Pullback) } \\
\text { la versus lb } \\
\text { (1) }\end{array}$ & $\begin{array}{c}\text { Intraobserver } \\
\text { Comparison } \\
\text { (repeated } \\
\text { Pullback) } \\
\text { la versus II } \\
\text { (2) }\end{array}$ & $\begin{array}{c}\text { Interobserer } \\
\text { Comparison } \\
\text { (same } \\
\text { Pullback) } \\
\text { la versus Ic } \\
\text { (3) }\end{array}$ & $\begin{array}{c}\text { ANOVA } \\
P\end{array}$ \\
\hline \multicolumn{5}{|l|}{ Vessel Geometrics } \\
\hline$\Delta$ Total Vessel Volume $\left(\mathrm{mm}^{3}\right)$ & $-1.25 \pm 3.8^{*}$ & $-1.67 \pm 4.9^{*}$ & $2.72 \pm 8.0^{*}$ & $<0.01^{\mathrm{a}}$ \\
\hline$\% \Delta$ Total Vessel Volume (\%) & $-0.40 \pm 1.0$ & $-0.42 \pm 1.2$ & $0.71 \pm 1.8$ & $<0.01^{\mathrm{b}}$ \\
\hline$\Delta$ Lumen Volume $\left(\mathrm{mm}^{3}\right)$ & $-1.06 \pm 3.5^{*}$ & $-1.28 \pm 5.1^{*}$ & $1.95 \pm 6.5^{*}$ & $<0.05^{\mathrm{c}}$ \\
\hline$\% \Delta$ Lumen Volume (\%) & $-0.48 \pm 1.4$ & $-0.52 \pm 1.8$ & $0.71 \pm 2.2$ & $<0.01^{d}$ \\
\hline$\Delta$ Plaque+media Volume $\left(\mathrm{mm}^{3}\right)$ & $-0.19 \pm 2.9^{*}$ & $-0.39 \pm 5.8^{*}$ & $0.76 \pm 7.5^{*}$ & 0.7 \\
\hline$\% \Delta$ Plaque+Media Volume (\%) & $-0.35 \pm 1.6$ & $-0.43 \pm 4.5$ & $0.89 \pm 5.0$ & 0.3 \\
\hline$\Delta$ Plaque Burden (\%) & $0.03 \pm 0.5^{*}$ & $0.05 \pm 1.2^{*}$ & $0.02 \pm 1.4^{*}$ & 0.9 \\
\hline$\% \Delta$ Plaque Burden (\%) & $0.04 \pm 1.4$ & $0.01 \pm 3.7$ & $0.20 \pm 4.2$ & 0.9 \\
\hline
\end{tabular}

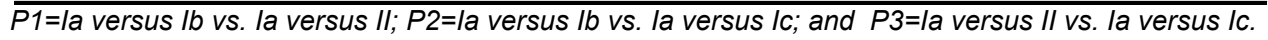
$a=P 2<0.05, P 3<0.01 ; b=P 2<0.01, P 3<0.01 ; c=P 3<0.05 ; d=P 2<0.05, P 3<0.05$.

*paired $t$-test la versus Ib; la versus II; and la versus Ic $p=N S$ for all. $\Delta=$ difference 

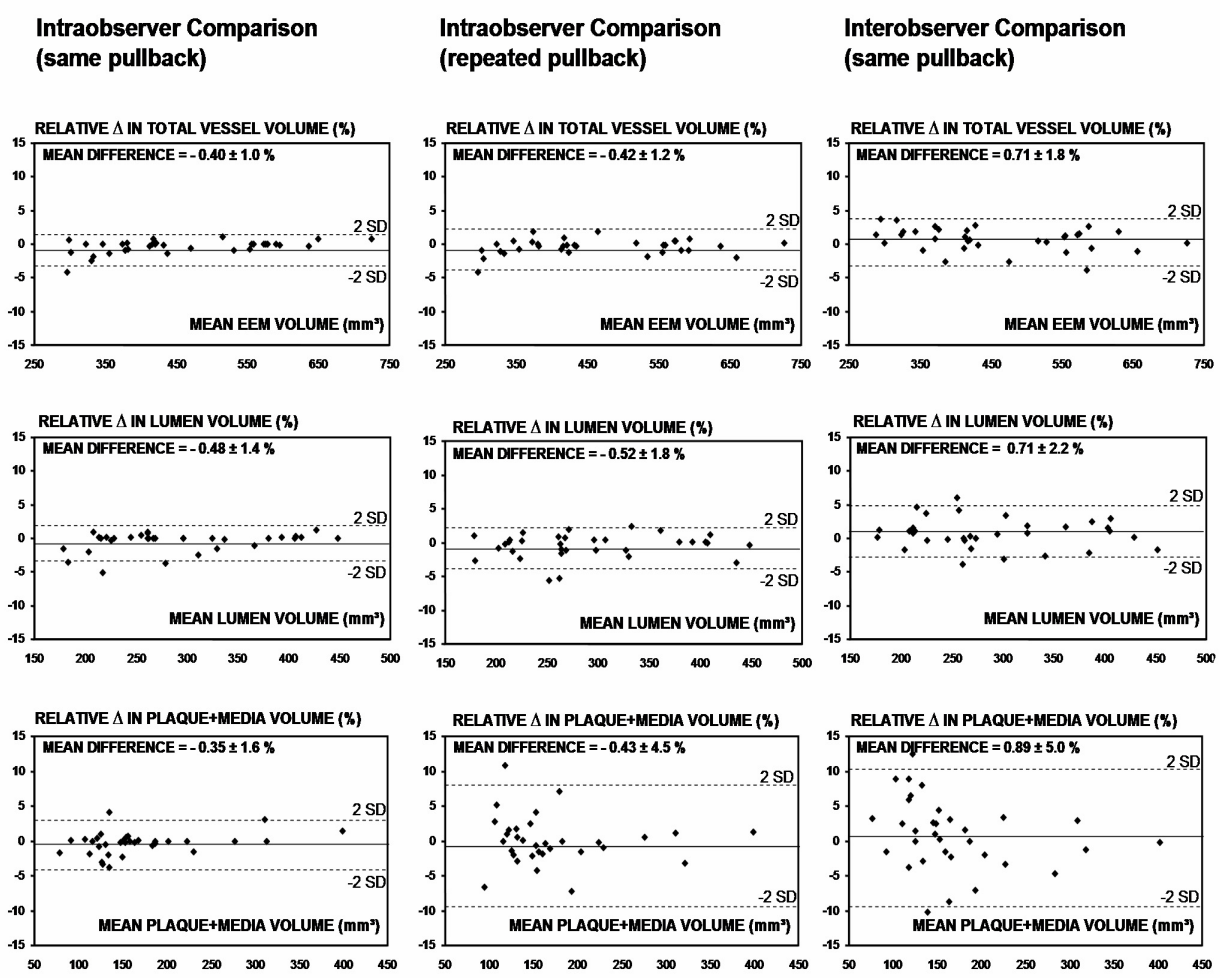

Figure 2. Agreement of repeated VH-IVUS measurements of geometrical vessel volumes. Bland-Altman plots of intra- (left, mid) and interobserver (right) comparisons. $\Delta=$ difference

Reproducibility of compositional RF-IVUS (intraobserver and interobserver comparison)

Data on the agreement and variability of repeated compositional measurements (absolute and/or relative differences) as presented in Table 2 and Figures 3 and 4 showed an acceptable reproducibility. Repeated compositional measurements were highly correlated for intraobserver comparison from the same pullback and repeated pullbacks, and for the interobserver comparison ( $r \geq 0.999, \geq 0.997$, and $\geq 0.995$; $P<0.001$ for all; data not shown). Of note, the interobserver comparison of compositional RF-IVUS measurements showed a higher variability than the intraobserver comparison from repeated IVUS pullbacks (Figure 3) with significantly higher relative differences for fibrous and fibro-lipidic volume $(P<0.01$ for both, Table 2). Necrotic-core volume showed on average the lowest measurement variability of all plaque components in both, intraobserver and interobserver comparisons. 
Table 2 (part 2). Intra- and interobserver measurement differences of volumetric vessel geometrics and plaque composition.

\begin{tabular}{|c|c|c|c|c|}
\hline & $\begin{array}{l}\text { Intraobserver } \\
\text { Comparsion } \\
\text { (same } \\
\text { Pullback) } \\
\text { la versus lb } \\
\text { (1) }\end{array}$ & $\begin{array}{c}\text { Intraobserver } \\
\text { Comparison } \\
\text { (repeated } \\
\text { Pullback) } \\
\text { la versus II } \\
\text { (2) }\end{array}$ & $\begin{array}{c}\text { Interobserer } \\
\text { Comparison } \\
\text { (same } \\
\text { Pullback) } \\
\text { la versus Ic } \\
\text { (3) }\end{array}$ & $\begin{array}{c}\text { ANOVA } \\
\mathbf{P}\end{array}$ \\
\hline \multicolumn{5}{|l|}{ Plaque Composition } \\
\hline$\Delta$ Fibrous Volume $\left(\mathrm{mm}^{3}\right)$ & $-0.04 \pm 0.7^{*}$ & $0.38 \pm 2.0^{*}$ & $-0.28 \pm 1.0^{*}$ & 0.1 \\
\hline$\% \Delta$ Fibrous Volume (\%) & $0.45 \pm 2.1$ & $1.40 \pm 4.1$ & $-1.60 \pm 4.9$ & $<0.01^{\mathrm{a}}$ \\
\hline$\Delta$ Fibrous (\%) & $0.18 \pm 0.6^{*}$ & $-0.02 \pm 1.2^{*}$ & $-0.37 \pm 1.5^{*}$ & 0.1 \\
\hline$\% \Delta$ Fibrous (\%) & $0.75 \pm 1.7$ & $0.24 \pm 2.8$ & $-1.00 \pm 4.2$ & 0.1 \\
\hline$\Delta$ Fibro-Lipidic Volume $\left(\mathrm{mm}^{3}\right)$ & $-0.04 \pm 0.3^{*}$ & $0.12 \pm 0.5^{*}$ & $0.17 \pm 0.8^{*}$ & 0.6 \\
\hline$\% \Delta$ Fibro-Lipidic Volume (\%) & $-1.12 \pm 4.9$ & $1.26 \pm 6.7$ & $3.85 \pm 8.2$ & $<0.01^{\text {b }}$ \\
\hline$\Delta$ Fibro-Lipidic (\%) & $0.11 \pm 0.8^{*}$ & $0.00 \pm 0.6^{*}$ & $0.27 \pm 0.9^{*}$ & 0.6 \\
\hline$\% \Delta$ Fibro-Lipidic (\%) & $0.27 \pm 8.2$ & $-0.07 \pm 5.8$ & $6.03 \pm 9.1$ & $<0.01^{\mathrm{C}}$ \\
\hline$\Delta$ Calcium Volume $\left(\mathrm{mm}^{3}\right)$ & $-0.01 \pm 0.3^{*}$ & $0.10 \pm 0.8^{*}$ & $-0.02 \pm 1.0^{*}$ & 0.6 \\
\hline$\% \Delta$ Calcium Volume (\%) & $-0.84 \pm 2.1$ & $2.66 \pm 7.4$ & $1.66 \pm 7.5$ & 0.1 \\
\hline$\Delta$ Calcium (\%) & $-0.13 \pm 0.4^{*}$ & $0.12 \pm 1.3^{*}$ & $0.45 \pm 1.5^{*}$ & 0.1 \\
\hline$\% \Delta$ Calcium (\%) & $-0.51 \pm 1.7$ & $1.52 \pm 6.7$ & $2.26 \pm 6.9$ & 0.1 \\
\hline$\Delta$ Necrotic-Core Volume $\left(\mathrm{mm}^{3}\right)$ & $-0.05 \pm 0.4^{*}$ & $0.18 \pm 0.8^{*}$ & $-0.21 \pm 0.8^{*}$ & 0.1 \\
\hline$\% \Delta$ Necrotic-Core Volume (\%) & $-0.22 \pm 1.8$ & $0.85 \pm 4.4$ & $-1.58 \pm 4.7$ & 0.1 \\
\hline$\Delta$ Necrotic-Core (\%) & $-0.03 \pm 0.4^{*}$ & $-0.11 \pm 1.0^{*}$ & $-0.23 \pm 0.8^{*}$ & 0.9 \\
\hline$\% \Delta$ Necrotic-Core (\%) & $0.07 \pm 1.8$ & $-0.34 \pm 3.6$ & $-0.90 \pm 3.4$ & 0.4 \\
\hline
\end{tabular}



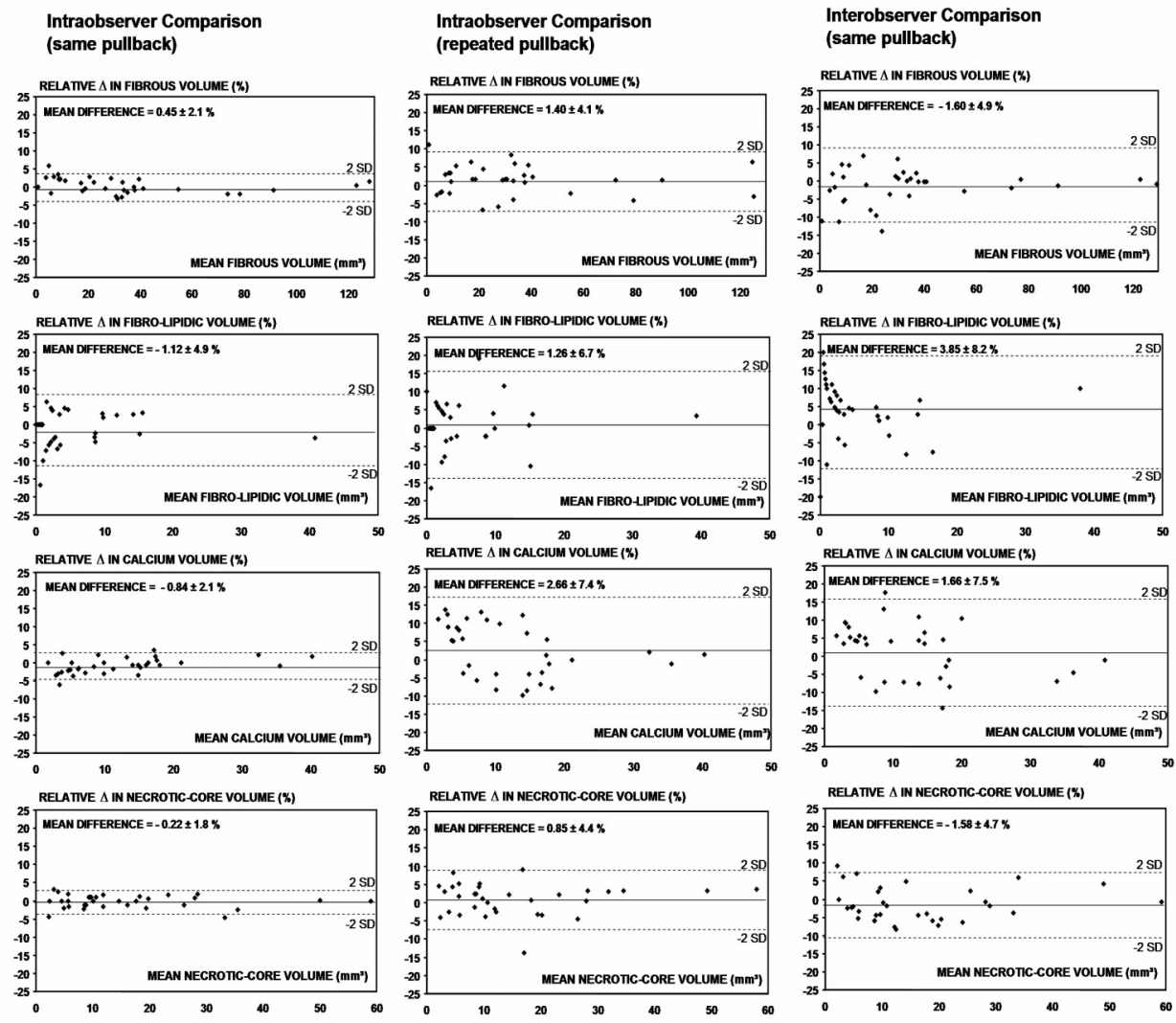

Figure 3. Agreement of repeated VH-IVUS measurements of compositional volumes. Bland-Altman plots of intra- (left, mid) and interobserver (right) comparisons. $\Delta=$ difference 


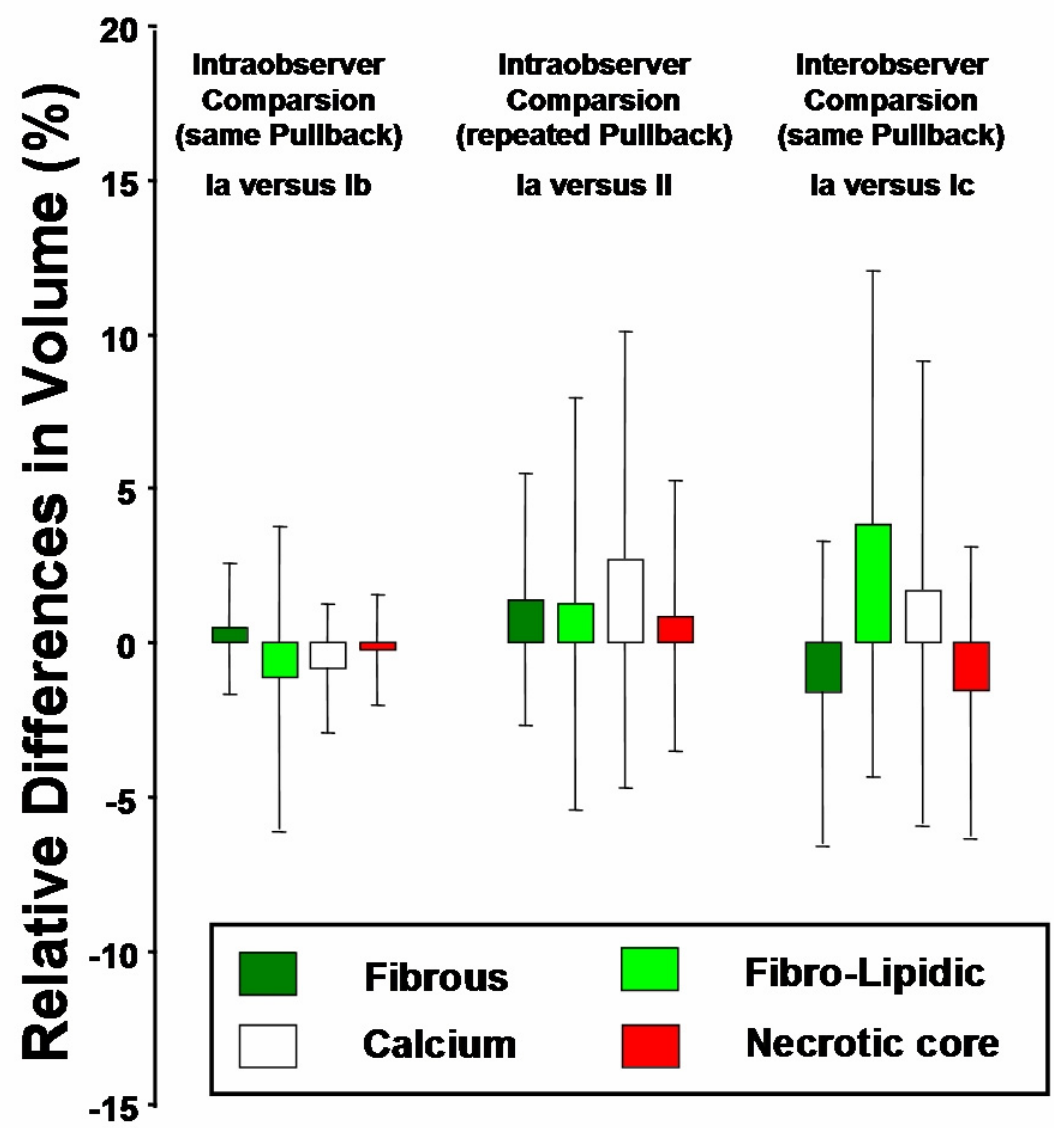

Figure 4. Relative differences of repeated compositional VH-IVUS volume measurements. The volumetric VH-IVUS measurements of plaque composition in intra(left and mid bars) and interobserver comparisons (right bars) are displayed (mean $\pm 1 \mathrm{SD}$ ). The paired t-test la versus $\mathrm{lb}$; la versus II; and la versus Ic showed no significant difference $(P=\mathrm{NS}$ for all). The necrotic-core volume showed on average the lowest measurement variability.

\section{Discussion}

Why measure changes in plaque composition?

Serial IVUS has become an established imaging method to measure effects of cardiovascular risk factors and drugs on coronary plaque dimensions. ${ }^{6-10,29-31}$ Plaque progression as measured by IVUS may be linked to a significant increase in risk of clinical events as predicted by established risk-scores. ${ }^{31}$ Therefore, IVUS 
derived surrogate endpoints as an alternative to clinical endpoints may expedite the process of drug development and testing. ${ }^{6}$ However, there is a contradiction between the significant clinical benefit of certain pharmacological interventions (e.g., statins) and the relatively small effect on plaque volume, which may be explained by a stabilizing effect on plaque composition. ${ }^{6,7,10,26,32}$

\section{Intravascular ultrasound radiofrequency analysis}

RF-IVUS is an imaging technique that permits the characterization and quantification of plaque components in vivo. ${ }^{16,17,23-25}$ Non-serial studies suggested a potential of RF-IVUS to detect high-risk coronary plaques (i.e., thin-cap fibroatheroma) and offered new insights into coronary pathology. ${ }^{18-22}$ Currently, large serial IVUS studies are on the way that assess with a volumetric RF-IVUS approach changes in plaque composition to analyze the effect of atherosclerosismodifying drugs on this new surrogate endpoint. ${ }^{33}$

\section{Rationale for the present study and main findings}

An important prerequisite for the use of RF-IVUS data as an endpoint in serial studies is the assessment of its reproducibility. ${ }^{17,34}$ Drug-induced effects on coronary arterial dimensions and plaque composition may remain relatively small. ${ }^{7-}$ ${ }^{10}$ To date there is a lack of volumetric reproducibility data simulating a serial RFIVUS study design (e.g. repeated pullbacks). ${ }^{17,34}$ Therefore, we assessed in the present study the reproducibility of repeated RF-IVUS analyses of vessel geometrics and plaque composition in 33 coronary segments. The relative intraobserver difference of geometrical and compositional volumetric analyses in repeated pullbacks was relatively low $(<0.6 \%$ for all geometric vessel volumes; $<1 \%$ for necrotic-core and $<3 \%$ for fibrous, fibro-lipidic, and calcific plaque components) and showed good limits of agreement. Of note, the interobserver comparison of RF-IVUS of geometrical and compositional measurements from the same pullback showed on average a somewhat higher variability than the intraobserver comparison of repeated pullbacks.

\section{Reproducibility of IVUS measurements}

The reproducibility of two-dimensional RF-IVUS has been tested by RodriguezGranillo et al. who compared repeated pullbacks from 16 coronary lesions. ${ }^{34}$ The relative intraobserver difference was $\leq 3 \%$ for the cross-sectional area measurements of vessel geometrics; in general compositional measurements were more variable than geometrical measurements. The relative intraobserver 
difference was $\leq 6 \%$ for necrotic-core area, and $8-13 \%$ for fibrous, fibro-lipidic, and calcium areas. ${ }^{34}$ Nevertheless, a volumetric IVUS approach is likely to result in a higher reproducibility. Kawasaki et al. reported intra- and interobserver variabilities of volumetric plaque compositional measurements ( $<3.6 \%$ for both) from 20 lesions from the same IVUS pullback by using the Integrated Backscatter IVUS technique, which is based on a different mathematical method compared to RF-IVUS or Virtual-Histology. ${ }^{17,26}$

Variability of compositional RF-IVUS analyses is related to the variability of geometrical vessel analysis. ${ }^{34,35}$ Tissue components mainly located in the center of an atherosclerotic plaque may be less sensitive to potential variation in border detection Factors of variability in geometrical vessel analyses in a serial study design are - besides the quality of the basic IVUS images - the quality of matching the region of interest. Matching the cross-sectional target slice from baseline and follow-up examination is much more difficult with a two-dimensional RF-IVUS approach. ${ }^{11,15}$ The problem of matching has been significantly reduced by the use of volumetric IVUS, which visualizes an entire coronary segment, permits identification of one or more landmarks (e.g., adjacent side-branches), and is the current standard method in serial IVUS trials that address atherosclerosismodifying strategies. ${ }^{7-10,15}$

In the present study, image acquisition was performed in an ECG-gated fashion. ECG-gated image acquisition prevents artifacts from cyclic movement of the IVUS catheter and the systolic-diastolic variation of the vessel dimensions which results in a higher reproducibility of geometrical IVUS measurements. ${ }^{13-15}$ In agreement with our results, Okkels et al. demonstrated a high reproducibility of vessel geometrical measurements from repeated pullbacks using a dedicated ECG-gated volumetric grey-scale IVUS technique. ${ }^{15}$

\section{The necrotic-core}

Necrotic-core tissue is the characteristic feature of so-called RF-IVUS derived thincap fibroatheroma (TCFA). ${ }^{16,17,18}$ Observational RF-IVUS studies demonstrated an association between RF-IVUS derived TCFA and both coronary and clinical instability. ${ }^{18,19,22}$ RF-IVUS derived TCFA is generally defined as at least three consecutive two-dimensional cross-sectional frames with $\geq 10 \%$ necrotic-core area without evident overlying fibrous tissue and a plaque burden area of $\geq 40 \%{ }^{18}$ Because of the two-dimensional nature of the definition of RF-IVUS derived TCFA, serial assessment may be difficult as outlined above; this may result in a higher measurement variability. Besides, if the luminal border is shared by thrombus which is more likely in coronary instability - the measurement variability of plaque and lumen dimensions may be even higher, as thrombus may be misclassified as 
fibrous-tissue. $^{35}$

A volumetric parameter that is less dependent on matching problems is the RF-IVUS derived necrotic-core volume. From a pathophysiological point of view the necrotic core volume is a very interesting target, as both the necrotic core size and its longitudinal extend are related to the risk of plaque rupture that may trigger cardiovascular events as demonstrated by histopathological studies. ${ }^{4,5,36,37}$ In agreement with these findings, Hong et al. recently found a significantly higher RFIVUS derived necrotic-core volume in lesions of patients with acute coronary syndromes compared to patients with stable angina. ${ }^{22}$ In our study in patients with stable angina, necrotic-core volume showed on average the highest reproducibility of all plaque components. Based on pathophysiological considerations and the favorable measurement reproducibility, the parameter RF-IVUS derived necroticcore volume has the potential to become a major imaging target of future pharmacological intervention trials with IVUS. Nevertheless, the relation between serial changes of this parameter and clinical endpoints has to be demonstrated.

\section{Limitations}

To the best of our knowledge, this was the first study that tested the reproducibility of volumetric RF-IVUS. The number of coronary segments was limited but comparable to other studies that addressed the reproducibility of IVUS measurements. ${ }^{13-15,34,38,39}$ All patients had stable angina pectoris. Similar to other studies with IVUS and RF-IVUS, we excluded tortuous and severely calcified vessels which could have led to non-uniform pullbacks. ${ }^{34}$ We analyzed high quality RF-IVUS image runs with limited circumferential calcification which resulted in a relatively high accuracy of total vessel border detection. ${ }^{11,12}$ Therefore, measurement reproducibility may be somewhat lower in routine clinical IVUS cases; nevertheless, the selection criteria of target segments in serial IVUS studies are similar. ${ }^{7-10}$ Inter-catheter and inter-system variability was not addressed. ${ }^{34,38,39}$ The high accuracy of the off-line RF-IVUS analysis was achieved at the expense of manual correction of the automatically derived lumen and vessel contours; accordingly, our data may not be generalized to on-line application of RF-IVUS.

\section{Conclusions}

In mild-to-moderate atherosclerotic coronary artery disease, the reproducibility of volumetric compositional RF-IVUS measurements from the same pullback is relatively high, but lower than the reproducibility of geometrical IVUS measurements. Measurements from repeated pullbacks and by different observers show acceptable reproducibilities for the use in a serial study design; the 
volumetric measurement of the necrotic-core shows on average the highest reproducibility of the compositional RF-IVUS measurements.

\section{References}

1. Waters D, Craven TE, Lesperance J. Prognostic significance of progression of coronary atherosclerosis. Circulation 1993;87:1399-1401.

2. Ambrose JA, Tannenbaum MA, Alexopoulos D, et al. Angiographic progression of coronary artery disease and the development of myocardial infarction. J Am Coll Cardiol 1988;12:56-62.

3. Shah PK. Mechanisms of plaque vulnerability and rupture. J Am Coll Cardiol 2003;41:15-22.

4. Burke AP, Farb A, Malcom GT, et al. Coronary risk factors and plaque morphology in men with coronary disease who died suddenly. N Engl J Med 1997:336:1276-1282.

5. Kolodgie FD, Virmani R, Burke AP, et al. Pathologic assessment of the vulnerable human coronary plaque. Heart 2004;90:1385-1391.

6. Böse D, von Birgelen C, Erbel R. Intravascular ultrasound for the evaluation of therapies targeting coronary atherosclerosis. J Am Coll Cardiol 2007;49:925-932.

7. Schartl M, Bocksch W, Koschyk DH, et al. Use of intravascular ultrasound to compare effects of different strategies of lipid-lowering therapy on plaque volume and composition in patients with coronary artery disease. Circulation 2001;104:387-392.

8. Jensen LO, Thayssen P, Pedersen KE, et al. Regression of coronary atherosclerosis by simvastatin: a serial intravascular ultrasound study. Circulation 2004;110:265-270.

9. Nissen SE, Nicholls SJ, Sipahi I, et al. Effect of very high-intensity statin therapy on regression of coronary atherosclerosis: the ASTEROID trial. JAMA 2006;295:1556-1565.

10. Tardif JC, Grégoire J, L'Allier PL, et al. Effect of rHDL on Atherosclerosis-Safety and Efficacy (ERASE) Investigators. Effects of reconstituted high-density lipoprotein infusions on coronary atherosclerosis: a randomized controlled trial. JAMA 2007;297:1675-1682.

11. Mintz GS, Nissen SE, Anderson WD, et al. American College of Cardiology clinical expert consensus document on standards for acquisition, measurement and reporting of intravascular ultrasound studies (IVUS). J Am Coll Cardiol 2001;37:1478-1492.

12. Di Mario C, Görge G, Peters R, et al. Clinical application and image interpretation in intracoronary ultrasound. Study group on intracoronary imaging of the working group of coronary circulation and of the subgroup on intravascular ultrasound of the working group of echocardiography of the european society of cardiology. Eur Heart J 1998;19:207-229.

13. von Birgelen C, de Vrey EA, Mintz GS, et al. ECG-gated three-dimensional intravascular ultrasound: feasibility and reproducibility of the automated analysis of coronary lumen and atherosclerotic plaque dimensions in humans. Circulation 1997;96:2944-2952.

14. Bruining N, von Birgelen C, de Feyter PJ, et al. ECG-gated versus nongated three-dimensional intracoronary ultrasound analysis: implications for volumetric measurements. Cathet Cardiovasc Diagn 1998;43:254-260.

15. Jensen LO, Thayssen P. Accuracy of electrocardiographic-gated versus nongated volumetric intravascular ultrasound measurements of coronary arterial narrowing. Am J Cardiol 2007;99:279283.

16. König A, Klauss V. Virtual Histology. Heart 2007;93:977-982.

17. Mehta SK, McCrary JR, Frutkin AD, et al. Intravascular ultrasound radiofrequency analysis of coronary atherosclerosis: an emerging technology for assessment of vulnerable plaque. Eur Heart J 2007;28:1283-1288.

18. Rodriguez-Granillo GA, García-García HM, McFadden EP, et al. In vivo intravascular ultrasoundderived thin-cap fibroatheroma detection using ultrasound radiofrequency data analysis. J Am Coll Cardiol 2005;46:2038-2042. 
19. Rodriguez-Granillo GA, García-García HM, Valgimigli M, et al. Global characterization of coronary plaque rupture phenotype using three-vessel intravascular ultrasound radiofrequency data analysis. Eur Heart J 2006;27:1921-1927

20. Valgimigli M, Rodriguez-Granillo GA, Garcia-Garcia HM, et al. Distance from the ostium as an independent determinant of coronary plaque composition in vivo: an intravascular ultrasound study based radiofrequency data analysis in humans. Eur Heart J 2006;27:655-663.

21. Fuji K, Carlier SG, Mintz GS, et al. Association of plaque characterization by intravascular ultrasound virtual histology and arterial remodeling. Am J Cardiol 2005;96:1476-1483.

22. Hong MK, Mintz GS, Lee CW, et al. Comparison of virtual histology to intravascular ultrasound of culprit coronary lesions in acute coronary syndrome and target coronary lesions in stable angina pectoris. Am J Cardiol 2007;100:953-959.

23. Nair A, Margolis MP, Kuban BD, et al. Automated coronary plaque characterisation with intravascular ultrasound backscatter: ex vitro validation. Eurointerv 2007;3:113-120.

24. Nair A, Kuban BD, Tuzcu EM, et al. Coronary plaque classification with intravascular ultrasound radiofrequency data analysis. Circulation 2002; 106:2200-2206.

25. Nasu K, Tsuchikane E, Katoh $O$, et al. Accuracy of in vivo coronary plaque morphology assessment: a validation study of in vivo virtual histology compared with in vitro histopathology. $\mathrm{J}$ Am Coll Cardiol 2006; 47:2405-2412.

26. Kawasaki M, Sano K, Okubo M, et al. Volumetric quantitative analysis of tissue characteristics of coronary plaques after statin therapy using three-dimensional integrated backscatter intravascular ultrasound. J Am Coll Cardiol 2005;45:1946-1953.

27. von Birgelen $C$, de Feyter PJ, de Vrey EA, et al. Simpson's rule for the volumetric ultrasound assessment of atherosclerotic coronary arteries: a study with ECG-gated three-dimensional intravascular ultrasound. Coron Artery Dis 1997;8:363-369.

28. Bland JM, Altman DG. Statistical methods for assessing agreement between two methods of clinical measurement. Lancet 1986;2:307-310.

29. Hartmann M, von Birgelen C, Mintz GS, et al. Relation between lipoprotein(a) and fibrinogen and serial intravascular ultrasound plaque progression in left main coronary arteries. J Am Coll Cardiol 2006;48:446-452.

30. von Birgelen C, Hartmann M, Mintz GS, et al. Relation between progression and regression of atherosclerotic left main coronary artery disease and serum cholesterol levels as assessed with serial long-term ( $\geq 12$ months) follow-up intravascular ultrasound. Circulation 2003;108:2757-2762.

31. von Birgelen C, Hartmann M, Mintz GS, et al. Relationship between cardiovascular risk as predicted by established risk scores versus plaque progression as measured by serial intravascular ultrasound in left main coronary arteries. Circulation 2004;110:1579-1585.

32. Ambrose JA, D'Agate DJ. Classification of systemic therapies for potential stabilization of the vulnerable plaque to prevent acute myocardial infarction. Am J Cardiol 2005;95:379-382

33. www.clinicaltrials.gov/ct/show/NCT00268996 (November 2007)

34. Rodriguez-Granillo GA, Vaina S, García-García HM, et al. Reproducibility of intravascular ultrasound radiofrequency data analysis: implications for the design of longitudinal studies. Int $\mathrm{J}$ Cardiovasc Imaging 2006;22:621-631.

35. Frutkin AD, Metha SK, McCrary JR, et al. Limitations to the use of virtual histology-intravascular ultrasound to detect vulnerable plaque. Eur Heart J 2007;28:1783-1784

36. Virmani R, Burke AP, Farb A, et al. Pathology of the vulnerable plaque. J Am Coll Cardiol 2006;47:C13-18.

37. Burke AP, Virmani R, Galis Z, et al. 34th Bethesda conference: task force\#2-What is the pathologic basis for new atherosclerosis imaging techniques? J Am Coll Cardiol 2003;41:1874-1886

38. Hartmann M, von Birgelen C, Mintz GS, et al. Dedicated calibration formulas permit correction of differences between measurements by different IVUS devices as demonstrated in atherosclerotic human coronary arteries in vitro. Int J Cardiovasc Imaging 2006;22:605-613. 
39. Rodriguez-Granillo GA, Bruining N, McFadden E, et al. Geometrical validation of intravascular ultrasound radiofrequency data analysis (Virtual Histology) acquired with a $30 \mathrm{MHz}$ boston scientific corporation imaging catheter. Catheter Cardiovasc Interv 2005;66:514-518. 



\title{
Chapter 11
}

\author{
Serial Intravascular Ultrasound of \\ Coronary Atherosclerosis: An Update
}

Marc Hartmann, Jennifer Huisman, Mounir W.Z. Basalus, Gert K. van Houwelingen, Martin G. Stoel, Patrick M.J. Verhorst, Clemens von Birgelen 

Coronary atherosclerotic disease remains one of the leading causes of morbidity and mortality in western-lifestyle countries. Established preventive pharmacological therapies reduce cardiovascular event rates by $30-40 \%$, but given the significant residual cardiovascular risk there is need to develop novel cardiovascular therapies to achieve even greater risk reduction. ${ }^{1,2}$

Increasing attention has been focused on the appropriate role of serial intravascular ultrasound (IVUS) assessment of coronary plaques as surrogate endpoint in the development process of novel pharmacological concepts (Figure 1). ${ }^{3-5}$ Several trials evaluated the effect of anti-atherosclerotic drugs on coronary plaque geometrics or composition as assessed with serial IVUS imaging. ${ }^{7-22}$ This review gives an update on various serial intravascular ultrasound lessons that we have learned so far from both, observational and randomized IVUS studies with pharmacological interventions.

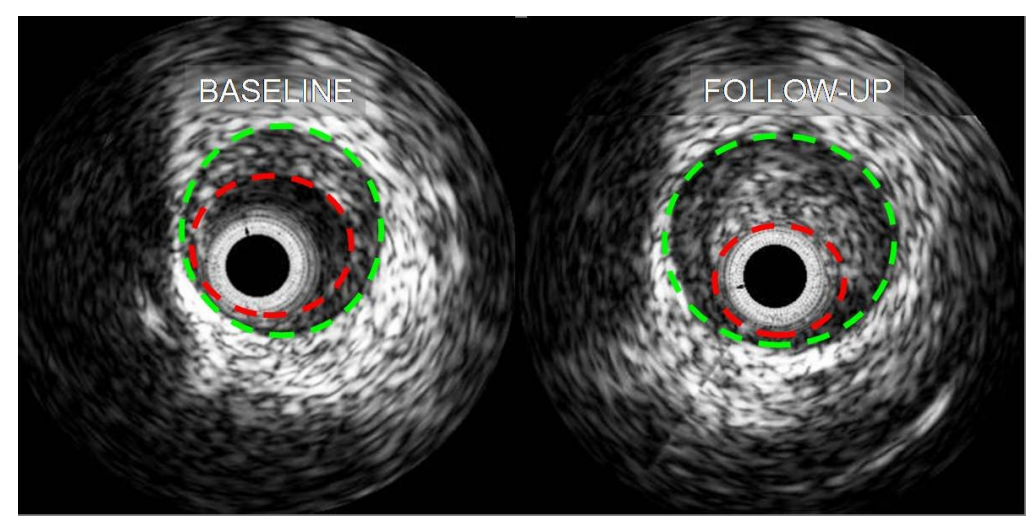

Figure 1. Serial IVUS assessment of coronary atherosclerosis progression.

\section{Serial IVUS - Cholesterol and Coronary Plaque Progression-Regression}

In 2003, the relation between low-density lipoprotein (LDL) cholesterol levels and coronary atherosclerosis progression was assessed with serial IVUS by our own group (18 \pm 9 months follow-up) (Figure 2 ) ${ }^{23}$ In this study, sixty patients treated by usual care including statins in the vast majority showed a direct linear relation between LDL-cholesterol levels and changes of plaque area in the left main stem $(r=0.41, p<0.001)$. An LDL-cholesterol value of $75 \mathrm{mg} / \mathrm{dl}$ was the threshold below which regression analysis predicted - on average - no increase of atherosclerotic plaque dimensions. ${ }^{23}$

During the following years two large-scale pharmacological intervention trials with statins used coronary plaque progression-regression as assessed with IVUS 
as an endpoint (Figure 2). ${ }^{11,18,24}$ In 2004, the LDL-cholesterol threshold of our observational study ${ }^{23}$ was essentially confirmed by the results of the Reversal of Atherosclerosis with Aggressive Lipid Lowering trial (REVERSAL), a prospective randomized study that tested the effect of 18 months of intensive versus moderate lipid-lowering therapy on coronary plaque progression. ${ }^{11}$ The REVERSAL trial demonstrated that atorvastatin $(80 \mathrm{mg})$ treatment to a mean LDL-cholesterol level of $79 \mathrm{mg} / \mathrm{dl}$ stopped progression of plaque volume. ${ }^{11}$ Our serial IVUS findings and the IVUS data of the REVERSAL trial are in good agreement with clinical trials that found an additional effect of intensive lipid-lowering therapy in high risk patients. ${ }^{25,26}$ Several smaller pharmacological intervention studies confirmed the impact of LDL-cholesterol lowering with statins on plaque progression as assessed with serial IVUS. ${ }^{7,9,10,26}$

In 2006, the Study to Evaluate the Effect of Rosuvastatin on Intravascular Ultrasound-Derived Coronary Atheroma Burden (ASTEROID) demonstrated for the first time that very high-intensity statin therapy with $10 \mathrm{mg}$ rosuvastatin to a mean LDL-cholesterol level of $60.8 \mathrm{mg} / \mathrm{dL}$ resulted in significant regression of atherosclerotic plaque volume as assessed with serial IVUS. ${ }^{18}$ However, the ASTEROID trial had no control group. ${ }^{18}$ Therefore, the ongoing Study of Coronary Atheroma by Intravascular UItrasound: Effect of Rosuvastatin Versus Atorvastatin (SATURN) compares the two statins in a randomized double blind multicenter trial with a serial IVUS design. ${ }^{27}$

Other novel cholesterol modifying agents were evaluated in serial IVUS studies. ${ }^{12,16}$ Cholesterol esterification by the enzyme acyl-coenzyme A cholesterol acyltransferase (ACAT) plays an important role in atherosclerotic plaque formation and inhibition of ACAT may therefore influence atherosclerosis progression. ${ }^{12}$ However, two serial IVUS studies showed no significant difference in plaque progression between patients treated with ACAT inhibitors versus placebo. ${ }^{12,16}$

Our observational study revealed (among other findings) a significant negative linear relation between high-density lipoprotein (HDL) cholesterol and atherosclerotic plaque progression. ${ }^{23} \mathrm{~A}$ small, but randomized serial IVUS trial showed that infusion of HDL-mimics (Apo-A1 Milano) induced during 5 weeks a significant regression of plaque volume in patients with acute coronary syndrome. ${ }^{8}$ However, another randomized, multicenter trial with serial IVUS examination found no significant regression of coronary atherosclerosis with infusion of HDL-mimics. ${ }^{19}$ Novel cardiovascular drugs as the cholesteryl-ester-transfer-protein-inhibitor torcetrapib achieved a substantial increase in HDL-cholesterol levels, but no significant decrease in coronary plaque progression as assessed with serial IVUS. ${ }^{17}$ The lack of efficacy of this drug may be related to the mechanism of action or to molecule-specific adverse effects (e.g., arterial hypertension).$^{17}$ Nevertheless, 
increase of HDL-cholesterol remains a valid target of preventive medicine and further studies are warranted. ${ }^{28}$
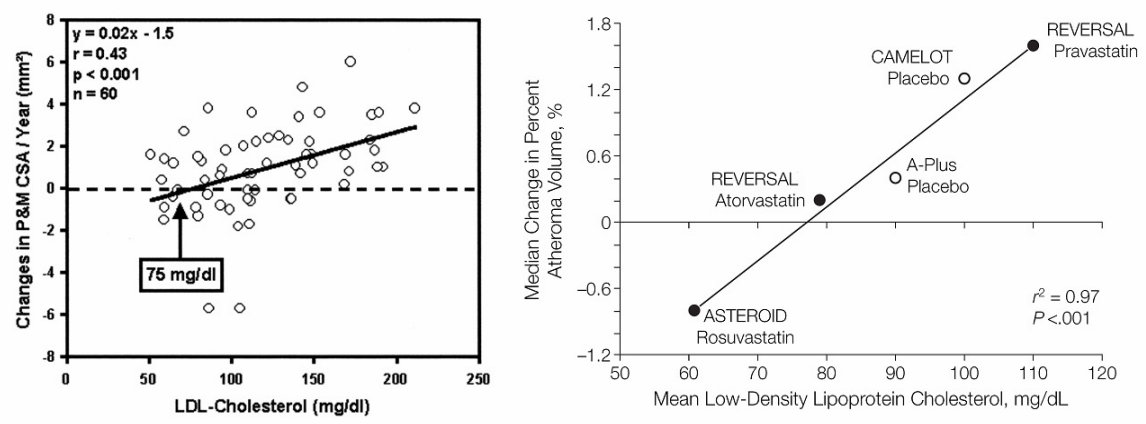

Figure 2. Low-density lipoprotein (LDL) cholesterol versus coronary plaque progressionregression as assessed with serial intravascular ultrasound. Demonstration of the positive significant relation between coronary plaque progression and LDL-cholesterol levels in a preliminary observational study (left graph, reproduced with permission from [23] (c) 2003 American Heart Association; all rights reserved). Confirmation of the relation between LDLcholesterol and coronary plaque progression by data derived from several large multicenter trials (right graph, reproduced with permission from [18] (c) 2006 American Medical Association; all rights reserved). An LDL-cholesterol value of 75 and $78 \mathrm{mg} / \mathrm{dl}$ were the thresholds below which regression analysis predicted, on average, no progression of atherosclerotic plaque, respectively (see both graphs).

\section{Serial IVUS - Surrogate Endpoint of Cardiovascular Risk?}

The assessment of morbidity and mortality as primary endpoints in conventional large-scale clinical trials is associated with a substantial financial burden. ${ }^{2-4}$ In order to develop novel anti-atherosclerotic therapies to reduce the remaining cardiovascular event risk, the superiority of novel agents has to be tested against established drugs in a head-to-head comparison, which requires a large study sample size and a relatively long duration of such studies. ${ }^{2-6}$ Surrogate endpoints, on the other hand, may allow to perform studies to test novel drugs with smaller study sample sizes and durations; consequently, the use of surrogate endpoints as an alternative to clinical endpoints may expedite the process of drug development and testing. Even complementary to clinical endpoints, the use of surrogate endpoints enables scientists to evaluate the potential benefits of novel antiatherosclerotic drugs before clinical endpoint data are available which may reduce costs and effort. ${ }^{2-6}$ 
However, an important pre-requisite of such studies is the evidence that a surrogate (imaging) endpoint reflects the clinical substitute: in the case of coronary atherosclerosis, this is cardiovascular events. Quantitative coronary angiography studies have shown that progressive obstruction of the coronary lumen - an indirect evidence of atherosclerotic plaque progression - is associated with an increased risk of adverse cardiovascular events. ${ }^{29-31}$ Therefore, it may be extrapolated that plaque progression, as quantified with serial IVUS - a direct evidence of atherosclerotic plaque progression - should show a similar or even stronger association with cardiovascular event risk. In the absence of a clear proof by a randomized study that directly addressed this question, there is evidence from several IVUS studies that provides support of the thesis that IVUS assessed plaque progression is a valid surrogate marker of cardiovascular event risk ${ }^{4}$ :

- In a non-serial IVUS study by Ricciardi et al. obstruction of the left main coronary artery, as detected by IVUS but angiographically silent, was an independent predictor of future adverse cardiac events. ${ }^{32}$ In 102 studied patients, major adverse cardiac events occurred in $38 \%$ of the cases during a follow-up of 29 months. By multivariate analysis, only IVUS assessed minimum lumen area and diabetes mellitus were significant independent predictors of these cardiac events. ${ }^{32}$

- The data provided by our group demonstrated that plaque progression as measured by IVUS was associated with a significantly increased risk of clinical events as predicted by established risk scores. ${ }^{33}$ During the follow-up period $30 \%$ actual adverse cardiovascular events occurred in patients with the greatest rate of plaque progression $(p<0.001$, Figure 3$) .{ }^{33}$ This data is obtained from a small retrospective analysis, nevertheless, recent prospective trials also provided evidence that supports the hypothesis that plaque progression as determined by IVUS is a valid predictive marker of cardiovascular events.

- Several clinical pharmacological intervention trials used IVUS assessed changes in atherosclerotic plaque dimensions as (additional) surrogate end points. $^{7-22}$ One trial reported that intensive antihypertensive therapy with amlodipine reduced coronary plaque progression as assessed with serial IVUS and adverse cardiovascular events. ${ }^{13}$ Patients with coronary artery disease and normal blood pressure $(n=1991)$ were randomized to receive either $10 \mathrm{mg}$ amlodipine, $20 \mathrm{mg}$ enalapril, or placebo. A subgroup of these patients $(n=274)$ had serial IVUS with a follow-up after 2 years. In the amlodipine group, there was no change in plaque volume $(p=0.31)$, while a non-significant increase in plaque volume was found in the enalapril group $(p=0.08)$, and a significant increase was seen in the placebo group $(p<0.001)$. In patients treated with amlodipine, the cardiovascular event-rate was significantly lower than in the 
placebo group ( 16.6 vs. $23.1 \%, p=0.003$ ); and the enalapril-treated group showed no significant difference from placebo $(20.3 \%, p=0.16) .{ }^{13}$ These results further underline the potential role of IVUS assessed plaque progression as a marker of cardiovascular event risk.

- The REVERSAL trial used the same treatment regimen as the clinical Pravastatin or Atorvastatin Evaluation and Infection Therapy (PROVE IT) trial, which reported a significantly greater reduction in cardiovascular events in patients with acute coronary syndromes after treatment with $80 \mathrm{mg} / \mathrm{day}$ atorvastatin for 2 years. ${ }^{11,25}$ The REVERSAL and PROVE IT studies were distinct studies, but when considered together their results provide further evidence that atherosclerotic progression measured by IVUS may most likely be predictive of an increased risk of cardiovascular events. ${ }^{11,25}$

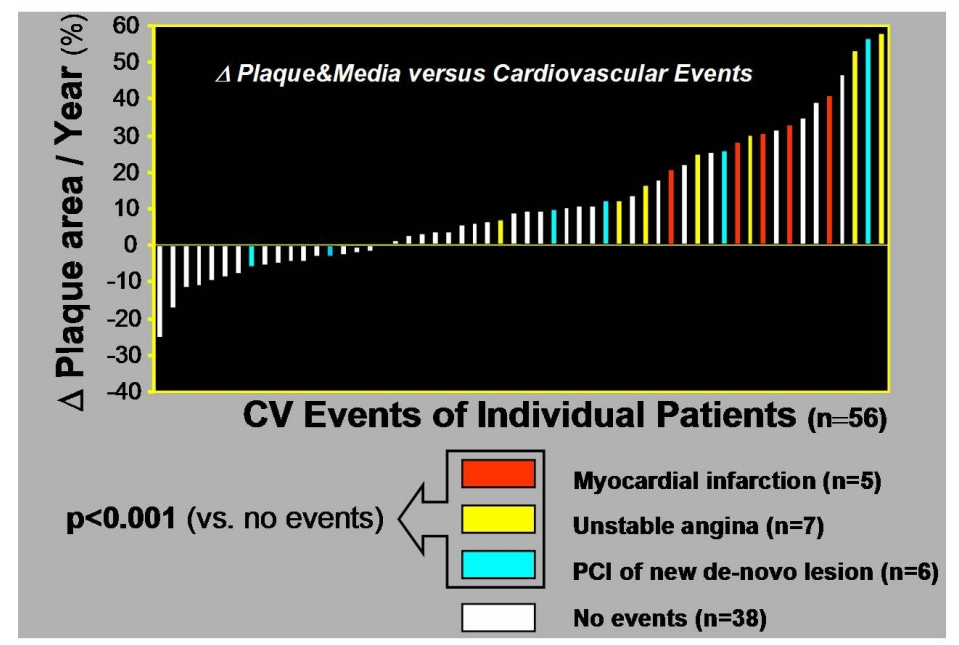

Figure 3. Serial IVUS assessed changes in coronary plaque dimensions versus actual adverse cardiovascular events (Modified and reproduced with permission from [33] (C) 2004 American Heart Association; all rights reserved).

These few examples underline the hypothesis that IVUS-detected progression of coronary atherosclerosis may be a valid surrogate marker of future cardiovascular events. Indeed, IVUS is a particularly suitable technique for the serial assessment of coronary atherosclerosis, given its highly accurate measurements, high measurement reproducibility, and its ability to detect mild, angiographically silent atherosclerotic disease that can be a precursor of future coronary events. ${ }^{34-39}$ As a result, several experimental anti-atherosclerotic drugs could be preliminary tested in serial IVUS studies before entering a large clinical endpoint trial. For example, the selective cannabinoid type 1 receptor antagonist rimonabant has positive 
metabolic effects in obese patients with a metabolic syndrome. ${ }^{40}$ In the recently published Strategy To Reduce Atherosclerosis Development Involving Administration of Rimonabant - The Intravascular Ultrasound Study (STRADIVARIUS), rimonabant failed to show a significant effect on the primary endpoint percent plaque volume, as assessed with serial IVUS after 18 months follow-up; the secondary endpoint total plaque volume, however, showed a significant reduction. ${ }^{21}$ The potential anti-atherosclerotic properties of other novel drugs (e.g., Peroxisome proliferator-activated receptor- $\gamma$, Lipoprotein-associated phospholipase A2 inhibitor) are being tested in ongoing serial IVUS trials. ${ }^{27}$

\section{Serial IVUS - Plaque Composition}

Both, the progression of atherosclerotic plaque size and unfavourable plaque tissue characteristics contribute to the overall risk of cardiovascular events. ${ }^{29-31,41-45}$ Progression-regression studies with serial IVUS reported a certain beneficial effect of anti-atherosclerotic pharmacological therapies on the progression of atherosclerotic plaque size - a geometrical measure., ${ }^{7,9-11,18,26}$ However, there seems to be a contradiction between the highly significant clinical benefit of certain pharmacological interventions (e.g., statins) and the relatively limited effect on plaque size by IVUS, which perhaps could be explained by a stabilizing effect on plaque composition. ${ }^{4-7,14,15,19,46-48}$

In a preliminary pharmacological intervention study, Schart et al. used computer-aided gray-scale IVUS analysis to detect changes of plaque echogenicity, which may reflect plaque composition. ${ }^{7}$ Lipid-lowering to LDL-cholesterol levels below $100 \mathrm{mg} / \mathrm{dl}$ with atorvastatin (compared with usual care) led to a significantly larger increase in plaque hyperechogenicity which is thought to reflect fibrous tissue; this may indicate plaque stabilization. ${ }^{7}$ Conventional grey-scale IVUS permits accurate quantification of the plaque and vessel dimensions for the assessment of plaque progression-regression. However, conventional IVUS has significant limitations in the assessment of plaque composition and the changes over time. . $-6,35-39,49-50^{-1}$

Therefore, a spectral analysis of IVUS radiofrequency (RF) data has been developed, which quantifies coronary plaque components (e.g., the necrotic core) with a high predictive accuracy as demonstrated in vitro and in vivo. ${ }^{51,52}$ Non-serial RF-IVUS studies provided interesting insights in the pathology of coronary atherosclerosis and demonstrated the ability to detect features of plaque vulnerability (e.g., necrotic core and thin-caped fibro-atheromas) in patients with unstable clinical presentation. ${ }^{53-57}$ Given its good measurement reproducibility, ${ }^{58,59}$ RF-IVUS seems to be a suitable image modality for the serial assessment of 
plaque composition during pharmacological intervention trials. ${ }^{14-15}$ Kawasaki et al. demonstrated plaque stabilization (decrease in necrotic and increase in fibrous tissue) with statin therapy in a serial RF-IVUS study. ${ }^{15}$ Currently, serial RF-IVUS studies are ongoing which analyze the effect of atherosclerosis-modifying drugs on both, plaque geometrics and plaque composition (e.g. Integrated Biomarker And Imaging Study - 2 trial). ${ }^{27}$

Specific RF-IVUS plaque characteristics are related to known risk factors of sudden cardiac death, and in doing so these characteristics may be associated with a worse prognosis. ${ }^{56}$ Nevertheless, the relation between serial changes of plaque composition as assessed with RF-IVUS and clinical endpoints has still to be demonstrated. ${ }^{49,50}$ Therefore, the ongoing Providing Regional Observations to Study Predictors of Events in the Coronary Tree (PROSPECT) trial has been designed, which tests the value of RF-IVUS plaque characteristics for prediction of future adverse cardiac events. ${ }^{27}$

\section{Serial IVUS - The Role Of Coronary Vessel Remodeling}

Surrogate imaging endpoints of trials which test anti-atherosclerotic drugs with serial IVUS focus on the assessment of both, progression-regression of atherosclerotic plaque size and change in plaque composition. But what about the total vessel size or the remodeling state of coronary lesions?

Non-serial IVUS observations found that positive remodeling and larger plaque areas were associated with unstable clinical presentations, whereas negative remodeling is more common in patients with stable clinical presentation. ${ }^{60}$ This association between the extent of remodeling and the clinical presentation may reflect a greater tendency of plaques with positive remodeling to cause unstable coronary syndromes, which was confirmed by other non-serial IVUS studies. ${ }^{61,62}$ Serial IVUS observations confirmed a broad spectrum of remodeling responses in mild-to-moderate atherosclerotic coronary lesions by assessing changes of the total vessel size over time. ${ }^{63}$

Reanalysis of serial IVUS data from trials that tested the effect of antiatherosclerotic drugs on plaque size (progression-regression) revealed some interesting insights into the serial remodeling behaviour (changes in coronary vessel size) during therapy. ${ }^{64-66}$ Schoenhagen et al. observed negative remodeling of the coronary vessel wall during plaque stabilizing therapy with statins that appeared to be related to their anti-inflammatory effects. ${ }^{66}$ Schartl et al. showed that the positive remodeling process is diminished in patients with plaque progression despite intensive lipid-lowering therapy. ${ }^{65}$ Tardif et al. concluded, based on their serial IVUS findings, that regression of atherosclerotic plaque is 
generally accompanied by negative remodeling without an increase in lumen dimensions ("reverse vascular remodeling"). ${ }^{64}$ It seems that - beside the reduction in plaque size (regression) - a shift of the remodeling pattern towards negative remodeling may be considered as a sign of plaque stabilization. ${ }^{64-66}$ However, the role of the remodeling state or behaviour as additional surrogate endpoint in serial IVUS trials has not yet been defined.

\section{Perspective}

As the global burden of cardiovascular disease increases, there is a need for surrogate endpoints to maximize efficacy in the evaluation of new antiatherosclerotic therapies. ${ }^{4}$ Most coronary events are the consequence of atherosclerotic plaque progression and vulnerability. ${ }^{29-31,41-45}$ Therefore, the measurement of changes in coronary plaque size or composition for potential prediction of clinical outcome has attracted much attention. ${ }^{4,33,56}$ The quantification of plaque dimensions with grey-scale IVUS (Figure 4A) is highly accurate, reproducible, and currently the gold standard for pharmacological progressionregression trials. ${ }^{5-6,7-22}$ Radiofrequency-based IVUS analysis (Figure 4B) provides the accurate and reproducible quantitative assessment of atherosclerotic plaque composition which permits to identify plaques with an increased vulnerability. As a result, serial RF-IVUS data are increasingly used in pharmacological intervention trials. ${ }^{5-6,49-50,27}$ As current non-invasive imaging techniques - such as multislice computed tomography or magnetic resonance tomography - still have several limitations for serial assessment of coronary atherosclerosis, invasive imaging with IVUS remains for the time being the gold standard. ${ }^{6}$

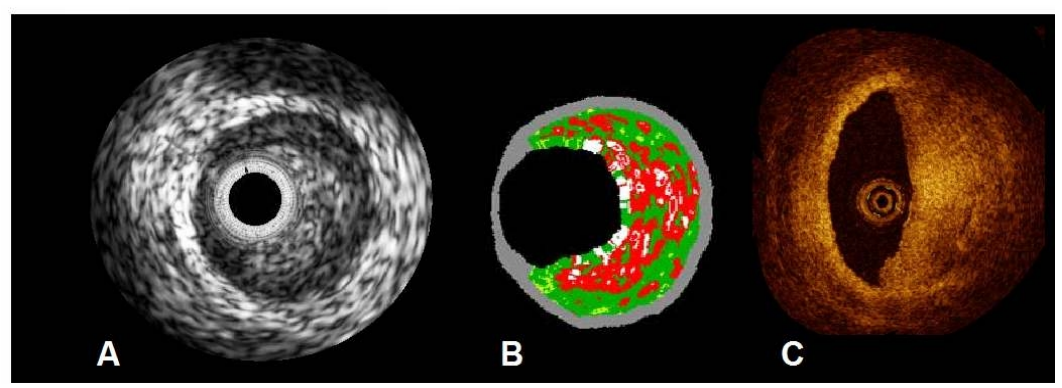

Figure 4. Technical progress may lead to the development of imaging catheters which may integrate grey-scale IVUS (A), radiofrequency-based IVUS (B) and optical coherence tomography $(\mathrm{C})$. 
There are some technical improvements of invasive imaging of coronary atherosclerosis. As pathological studies taught us, vulnerable plaques are generally characterized by a necrotic core and a thin fibrous cap $(<65 \mu \mathrm{m}) .{ }^{41-45} \mathrm{RF}$ IVUS can visualize and measure the necrotic core but is unable to visualize the thin fibrous cap (because of its limited resolution of approximately 100 $\mu \mathrm{m}$ ) ${ }^{49,50,70}$ But this thin fibrous cap is a defining feature of plaques "prone to rupture" ${ }^{41-45}$ Optical coherence tomography (OCT, Figure $4 \mathrm{C}$ ) provides high resolution (approximately $10 \mu \mathrm{m})$ images of intimal structures including the accurate visualization of thin fibrous caps. ${ }^{6,69,70}$ For instance, serial OCT showed a significant increase of the thin fibrous cap thickness during statin therapy. ${ }^{69}$ However, OCT is unable to reliably detect a large and deep necrotic core, because its penetrance is limited to a depth of approximately $1-2 \mathrm{~mm} .{ }^{6,70}$ Recently, Sawada et al. demonstrated a more effective detection of vulnerable plaques by combined use of the complementary imaging modalities OCT plus IVUS. ${ }^{70}$ The development of one image catheter which may offer the simultaneous use of both modalities (Figure 4A-C) during one pullback through a coronary artery is currently in progress. Such technical advances will provide new insights into the natural history of atherosclerosis and may permit optimisation of surrogate endpoints for future pharmacological intervention trials that aim at further reduction of cardiovascular morbidity and/or mortality.

\section{References}

1. Brown MS, Goldstein JL. Heart attacks: gone with the century? Science 1996;272:629.

2. Libby P. The forgotten majority: unfinished business in cardiovascular risk reduction. J Am Coll Cardiol 2005;46:1225-1228.

3. Loscalzo J. Clinical trials in cardiovascular medicine in an era of marginal benefit, bias, and hyperbole. Circulation 2005;112:3026-3029.

4. Böse D, von Birgelen C, Erbel R. Intravascular ultrasound for the evaluation of therapies targeting coronary atherosclerosis. J Am Coll Cardiol 2007;49:925-932.

5. Kastelein JJ, de Groot E. Ultrasound imaging techniques for the evaluation of cardiovascular therapies. Eur Heart J 2008;29:849-858.

6. Lindsay AC, Choudhury RP. Form to function: current and future roles for atherosclerosis imaging in drug development. Nat Rev Drug Discov 2008;7:517-529.

7. Schartl M, Bocksch W, Koschyk DH, et al. Use of intravascular ultrasound to compare effects of different strategies of lipid-lowering therapy on plaque volume and composition in patients with coronary artery disease. Circulation 2001;104:387-392.

8. Nissen SE, Tsunoda T, Tuzcu EM, et al. Effect of recombinant ApoA-I Milano on coronary atherosclerosis in patients with acute coronary syndromes: a randomized controlled trial. JAMA 2003;290:2292-2300.

9. Jensen LO, Thayssen P, Pederson KE, et al. Regression of coronary atherosclerosis by simvastatin: a serial intravascular ultrasound study. Circulation 2004;110:265-270.

10. Okazaki S, Yokoyama T, Miyauchi K, et al. Early statin treatment in patients with acute coronary 
syndrome: demonstration of the beneficial effect on atherosclerotic lesions by serial volumetric intravascular ultrasound analysis during half a year after coronary event: the ESTABLISH Study. Circulation 2004;110:1061-1068.

11. Nissen SE, Tuzcu EM, Schoenhagen $P$, et al. Effect of intensive compared with moderate lipidlowering therapy on progression of coronary atherosclerosis A randomized controlled trial. JAMA 2004;291:1071-1080.

12. Tardif JC, Grégoire J, L'Allier PL, et al. Effects of the acyl coenzyme A: cholesterol acyltransferase inhibitor avasimibe on human atherosclerotic lesions. Circulation 2004;110:3372-3377.

13. Nissen SE, Tuzcu EM, Libby $P$, et al. Effect of antihypertensive agents on cardiovascular events in patients with coronary disease and normal blood pressure: the CAMELOT study: a randomized controlled trial. JAMA 2004;292:2217-2225.

14. Yokoyama M, Komiyama N, Courtney BK, et al. Plasma low-density lipoprotein reduction and structural effects on coronary atherosclerotic plaques by atorvastatin as clinically assessed with intravascular ultrasound radio-frequency signal analysis: a randomized prospective study. Am Heart J 2005;150:287.e1-287.e7.

15. Kawasaki M, Sano K, Okubo M, et al. Volumetric quantitative analysis of tissue characteristics of coronary plaques after statin therapy using three-dimensional integrated backscatter intravascular ultrasound. J Am Coll Cardiol 2005;45:1946-1953.

16. Nissen SE, Tuzcu EM, Brewer HB, et al. Effect of ACAT inhibition on the progression of coronary atherosclerosis. N Engl J Med 2006;354:1253-1263.

17. Nissen SE, Tardif JC, Nicholls SJ, et al. Effect of torcetrapib on the progression of coronary atherosclerosis. N Engl J Med 2007;356:1304-1316.

18. Nissen SE, Nicholls SJ, Sipahi I, et al. Effect of very high-intensity statin therapy on regression of coronary atherosclerosis: the ASTEROID trial. JAMA 2006;295:1556-1565.

19. Tardif JC, Grégoire J, L'Allier PL, et al. Effect of rHDL on atherosclerosis-safety and efficacy (ERASE) investigators. Effects of reconstituted high-density lipoprotein infusions on coronary atherosclerosis: a randomized controlled trial. JAMA 2007;297:1675-1682.

20. Rodriguez-Granillo GA, Vos J, Bruining N, et al. Investigators of the EUROPA Study. Long-term effect of perindopril on coronary atherosclerosis progression (from the perindopril's prospective effect on coronary atherosclerosis by angiography and intravascular ultrasound evaluation [PERSPECTIVE] study). Am J Cardiol 2007;100:159-163.

21. Nissen SE, Nicholls SJ, Wolski K, et al. Effect of rimonabant on progression of atherosclerosis in patients with abdominal obesity and coronary artery disease: the STRADIVARIUS randomized controlled trial. JAMA 2008;299:1547-1560.

22. Tardif JC, Grégoire J, L'Allier PL, et al. Effects of the antioxidant succinobucol (AGI-1067) on human atherosclerosis in a randomized clinical trial. Atherosclerosis 2008;197:480-486.

23. von Birgelen C, Hartmann M, Mintz GS, et al. Relation between progression and regression of atherosclerotic left main coronary artery disease and serum cholesterol levels as assessed with serial long-term ( $\geq 12$ months) follow-up intravascular ultrasound. Circulation 2003;108:2757-2762.

24. Larosa JC, Grundy SM, Waters DD, et al. Intensive lipid lowering with atorvastatin in patients with stable coronary disease. N Engl J Med 2005;352:1425-1435.

25. Cannon $\mathrm{CP}$, Braunwald $\mathrm{E}, \mathrm{McC}$ abe $\mathrm{CH}$, et al. Intensive versus moderate lipid lowering with statins after acute coronary syndromes. N Engl J Med 2004;350:1495-1504.

26. Rodriguez-Granillo GA, Agostoni P, Garcia-Garcia HM, et al. Meta-analysis of the studies assessing temporal changes in coronary plaque volume using intravascular ultrasound. Am J Cardiol 2007;99:5-10.

27. www.clinicaltrials.gov

28. Nicholls SJ, Tuzcu EM, Sipahi I, et al. Statins, high-density lipoprotein cholesterol, and regression of coronary atherosclerosis. JAMA 2007;297:499-508.

29. Azen SP, Mack WJ, Cashin-Hemphill L, et al. Progression of coronary artery disease predicts 
clinical coronary events long-term follow-up from the cholesterol lowering atherosclerosis study. Circulation 1996:93:34-41.

30. Waters D, Craven TE, Lesperance J. Prognostic significance of progression of coronary atherosclerosis. Circulation 1993;87:1399-1401.

31. Ambrose JA, Tannenbaum MA, Alexopoulos D, et al. Angiographic progression of coronary artery disease and the development of myocardial infarction. J Am Coll Cardiol 1988;12:56-62.

32. Ricciardi MJ, Meyers S, Choi K, et al. Angiographically silent left main disease detected by intravascular ultrasound: a marker for future adverse cardiac events. Am Heart J 2003:146:507512.

33. von Birgelen C, Hartmann M, Mintz GS, et al. Relationship between cardiovascular risk as predicted by established risk scores versus plaque progression as measured by serial intravascular ultrasound in left main coronary arteries. Circulation 2004;110:1579-1585.

34. Berry C, L'Allier PL, Grégoire J, et al. Comparison of intravascular ultrasound and quantitative coronary angiography for the assessment of coronary artery disease progression. Circulation 2007;115:1851-1857.

35. Mintz GS, Nissen SE, Anderson WD, et al. American College of Cardiology clinical expert consensus document on standards for acquisition, measurement and reporting of intravascular ultrasound studies (IVUS). J Am Coll Cardiol 2001;37:1478-1492.

36. Di Mario C, Görge G, Peters R, et al. Clinical application and image interpretation in intracoronary ultrasound. Study group on intracoronary imaging of the working group of coronary circulation and of the subgroup on intravascular ultrasound of the working group of echocardiography of the European Society of Cardiology. Eur Heart J 1998;19:207-229.

37. von Birgelen C, de Very EA, Mintz GS, et al. ECG-gated three-dimensional intravascular ultrasound: feasibility and reproducibility of the automated analysis of coronary lumen and atherosclerotic plaque dimensions in humans. Circulation 1997;96:2944-2952.

38. von Birgelen C, de Feyter PJ, de Vrey EA, et al. Simpson's rule for the volumetric ultrasound assessment of atherosclerotic coronary arteries: a study with ECG-gated three-dimensional intravascular ultrasound. Coron Artery Dis 1997;8:363-369.

39. Jensen LO, Thayssen P, Pedersen KE, et al. Low variation and high reproducibility in plaque volume with intravascular ultrasound. Int J Cardiol 2004;97:463-469.

40. Pi-Sunyer FX, Aronne LJ, Heshmati HM, et al. Effect of rimonabant, a cannabinoid-1 receptor blocker, on weight and cardiometabolic risk factors in overweight or obese patients: RIO-North America: a randomized controlled trial. JAMA 2006;295:761-775.

41. Shah PK. Mechanisms of plaque vulnerability and rupture. J Am Coll Cardiol 2003;41:15-22.

42. Burke AP, Farb A, Malcom GT, et al. Coronary risk factors and plaque morphology in men with coronary disease who died suddenly. N Engl J Med 1997:336:1276-1282.

43. Kolodgie FD, Virmani R, Burke AP, et al. Pathologic assessment of the vulnerable human coronary plaque. Heart 2004;90:1385-1391.

44. Virmani R, Burke AP, Farb A, et al. Pathology of the vulnerable plaque. J Am Coll Cardiol 2006;47:C13-18.

45. Burke AP, Virmani R, Galis Z, et al. 34th Bethesda conference: task force\#2-What is the pathologic basis for new atherosclerosis imaging techniques? J Am Coll Cardiol 2003;41:1874-1886

46. Hong MK, Mintz GS, Lee CW, et al. Serial intravascular ultrasound evidence of both plaque stabilization and lesion progression in patients with ruptured coronary plaques: effects of statin therapy on ruptured coronary plaque. Atherosclerosis 2007;191:107-114.

47. Ambrose JA, D'Agate DJ. Classification of systemic therapies for potential stabilization of the vulnerable plaque to prevent acute myocardial infarction. Am J Cardiol 2005;95:379-382

48. Libby $P$, Sasiela W. Plaque stabilization: Can we turn theory into evidence? Am J Cardiol 2006;98(11A):26P-33P.

49. König A, Klauss V. Virtual Histology. Heart 2007;93:977-982. 
50. Mehta SK, McCrary JR, Frutkin AD, et al. Intravascular ultrasound radiofrequency analysis of coronary atherosclerosis: an emerging technology for assessment of vulnerable plaque. Eur Heart J 2007;28:1283-1288.

51. Nair A, Kuban BD, Tuzcu EM, et al. Coronary plaque classification with intravascular ultrasound radiofrequency data analysis. Circulation 2002; 106:2200-2206.

52. Nasu K, Tsuchikane E, Katoh $\mathrm{O}$, et al. Accuracy of in vivo coronary plaque morphology assessment: a validation study of in vivo virtual histology compared with in vitro histopathology. $\mathrm{J}$ Am Coll Cardiol 2006;47:2405-2412.

53. Hong MK, Mintz GS, Lee CW, et al. Comparison of virtual histology to intravascular ultrasound of culprit coronary lesions in acute coronary syndrome and target coronary lesions in stable angina pectoris. Am J Cardiol 2007;100:953-959.

54. Rodriguez-Granillo GA, García-García HM, McFadden EP, et al. In vivo intravascular ultrasoundderived thin-cap fibroatheroma detection using ultrasound radiofrequency data analysis. J Am Coll Cardiol 2005;46:2038-2042.

55. Rodriguez-Granillo GA, García-García HM, Valgimigli M, et al. Global characterization of coronary plaque rupture phenotype using three-vessel intravascular ultrasound radiofrequency data analysis. Eur Heart J 2006;27:1921-1927

56. Missel E, Mintz GS, Carlier SG, et al. In vivo virtual histology intravascular ultrasound correlates of risk factors for sudden coronary death in men: results from the prospective, multi-centre virtual histology intravascular ultrasound registry. Eur Heart J 2008;DOI:10.1093/eurhearti/ehn293.

57. Missel E, Mintz GS, Carlier SG, et al. Necrotic core and its ratio to dense calcium are predictors of high-risk non-ST-elevation acute coronary syndrome. Am J Cardiol 2008;101:573-578.

58. Rodriguez-Granillo GA, Vaina S, García-García HM, et al. Reproducibility of intravascular ultrasound radiofrequency data analysis: implications for the design of longitudinal studies. Int $\mathrm{J}$ Cardiovasc Imaging 2006;22:621-631.

59. Hartmann M,Mattern ESK,Huisman J, et al. Reproducibility of volumetric intravascular ultrasound radiofrequency-based analysis of coronary plaque composition in vivo. Int $\mathrm{J}$ Cardiovasc Imaging 2008; DOI:10.1007/s10554-008-9338-9.

60. Schoenhagen P, Ziada KM, Kapadia SR, et al. Extent and direction of arterial remodeling in stable versus unstable coronary syndromes: an intravascular ultrasound study. Circulation 2000;101:598603.

61. Schoenhagen P, Ziada KM, Vince DG, et al. Arterial remodeling and coronary artery disease: the concept of "dilated" versus "obstructive" coronary atherosclerosis. J Am Coll Cardiol 2001;38:297306.

62. von Birgelen C, Klinkhart W, Mintz GS, et al. Plaque distribution and vascular remodeling of ruptured and nonruptured coronary plaques in the same vessel: an intravascular ultrasound study in vivo. J Am Coll Cardiol 2001;37:1864-1870.

63. von Birgelen C, Hartmann M, Mintz GS, et al. Spectrum of remodeling behavior observed with serial long-term ( $\geq 12$ months) follow-up intravascular ultrasound studies in left main coronary arteries. Am J Cardiol 2004;93:1107-1113.

64. Tardif JC, Grégoire J, L'Allier PL, et al. Effect of atherosclerotic regression on total luminal size of coronary arteries as determined by intravascular ultrasound. Am J Cardiol 2006;98:23-27.

65. Schartl M, Bocksch W, Fateh-Moghadam S. Effects of lipid-lowering therapy on coronary artery remodeling. Coron Artery Dis 2004;15:45-51.

66. Schoenhagen P, Tuzcu EM, Apperson-Hansen C, et al. Determinants of arterial wall remodeling during lipid-lowering therapy: serial intravascular ultrasound observations from the reversal of atherosclerosis with aggressive lipid lowering therapy (REVERSAL) trial. Circulation 2006;113:2826-2834.

67. Frutkin AD, Metha SK, McCrary JR, et al. Limitations to the use of virtual histology-intravascular ultrasound to detect vulnerable plaque. Eur Heart J 2007;28:1783-1784 
68. Yamaguchi T, Terashima M, Akasaka T, et al. Safety and feasibility of an intravascular optical coherence tomography image wire system in the clinical setting. Am J Cardiol 2008;101:562-567.

69. Takarada S, Imanishi T, Kubo T, et al. Effect of statin therapy on coronary fibrous-cap thickness in patients with acute coronary syndrome: Assessment by optical coherence tomography study. Atherosclerosis 2008; DOI:10.1016/j.atherosclerosis.2008.05.014.

70. Sawada T, Shite J, Garcia-Garcia HM, et al. Feasibility of combined use of intravascular ultrasound radiofrequency data analysis and optical coherence tomography for detecting thin-cap fibroatheroma. Eur Heart J 2008;29:1136-1146. 



\section{Summary and Conclusions}





\section{Summary and Conclusions}

Cardiovascular disease is a major cause of morbidity and mortality in countries with western lifestyle. Reliable quantitative assessment of the extent of coronary atherosclerosis during the natural history of coronary artery disease and following therapeutic interventions is important to evaluate disease progression and antiatherosclerotic therapies.

Coronary angiography visualizes the luminal silhouette but not the vessel wall where the atherosclerotic disease process is actually located. Intravascular ultrasound (IVUS), however, provides transmural tomographic images of the coronary artery and direct measurements of lumen, plaque, and vessel dimensions.

Serial IVUS measurements allow to quantify the extent of coronary plaque progression or regression and changes in total vessel dimensions (remodeling process) as introduced in Chapter 1. The Chapters 2-4 of this thesis focus on the relation between serum parameters and progression-regression of coronary atherosclerosis in the left main stem as assessed with serial IVUS. In addition, Chapter 5 explores the relation between the risk of cardiovascular events and IVUS assessed plaque progression-regression. The Chapters 6-8 investigate the remodeling behaviour of coronary lesions in a serial IVUS design by providing (1) evidence of serial remodeling (Chapter 6), (2) the validation of the non-serial assessment of coronary remodeling (Chapter 7), and (3) the validation of Glagov's histopathological remodeling hypothesis. Chapter 9 assesses measurement differences between different IVUS devices in vitro and validated dedicated calibration formulas for correction. Chapter 10 focuses on volumetric radiofrequency-based IVUS analysis of atherosclerotic plaque composition and evaluates the reproducibility of this technique for the use in studies with a serial design. Finally, Chapter 11 reviews the current knowledge of coronary atherosclerosis that was been gained from observational and randomized pharmacological intervention trials with serial IVUS.

Chapter 2 presents serial IVUS data of 60 left main coronary arteries which were studied $18 \pm 9$ months apart to evaluate the progression or regression of atherosclerotic plaques in relation to serum cholesterol levels. There was a positive linear relation between low-density lipoprotein (LDL) cholesterol and changes in plaque cross-sectional area ( $r=0.41, p<0.0001)$ with (2) an LDL cholesterol value of $75 \mathrm{mg} / \mathrm{dL}$ as the cut-off when regression analysis predicted on average no plaque progression; (3) a negative linear relation between high-density lipoprotein cholesterol and changes in plaque area; (4) an negative linear relation between LDL cholesterol and changes in lumen area. 
This chapter extends our knowledge about the clinical relation between serum lipids and the risk of coronary events by directly demonstrating the relation between serum cholesterol values versus serial changes in coronary plaque dimensions. Furthermore, this chapter underlines the therapeutic approach of aggressive lipid-lowering in patients with established coronary artery disease.

In Chapter 3 the relation between cholesterol values and plaque progression as assessed with serial IVUS in left main stems is assessed at different stages of age. Patients $(n=60)$ were divided in tertiles according to their age: tertile 1 had a mean age of 48 years ( 33 to 55 year), tertile 2 had a mean age of 58 years (55 to 61 year), and tertile 3 had a mean age of 66 years (61 to 83 year). There was a positive linear relation between LDL cholesterol and changes in plaque area in all tertiles, which was statistically significant in tertiles 2 and $3(p<0.02)$ and showed a strong trend in tertile 1 . The estimated LDL cholesterol threshold corresponding to no plaque progression, as determined by regression analysis, was $80 \mathrm{mg} / \mathrm{dl}$ in all tertiles.

This chapter suggests a potential clinical benefit of (aggressive) lipid lowering in older patients by demonstrating a persistent relation between $L D L$ cholesterol and plaque progression during aging.

In Chapter 4 we assess the relation between the cardiovascular risk factors lipoprotein $(a)[L p(a)]$ and fibrinogen versus coronary plaque progression and adverse cardiovascular events. There was a positive correlation between $L p(a)$, fibrinogen, and changes in plaque area. Patients with plaque progression had higher $L p(a)$ and fibrinogen levels than patients with plaque regression. $L p(a)$, but not fibrinogen, was independently associated with plaque progression $(p<0.001)$. Patients with adverse cardiovascular events had higher $L p(a)$ and fibrinogen levels $(p<0.0001)$. $L p(a)$ and fibrinogen were independently associated with adverse cardiovascular events.

This chapter demonstrates the relation between classic and novel cardiovascular risk factors, atherosclerotic plaque growth and subsequent cardiovascular events.

Chapter 5 compares both, (1) estimated cardiovascular risk (derived from 3 established risk scores for primary prevention - PROCAM, SCORE, and Framingham) and (2) actual cardiovascular events versus plaque progression as assessed with serial IVUS in atherosclerotic left main coronary arteries. Patients at highest estimated risk of events showed greater plaque progression than patients at lower risk. There were positive linear relationships between the risk of clinical events and plaque progression. During follow-up, 18 patients suffered from 
adverse cardiovascular events; these patients had plaque progression that was significantly greater than that of patients without events $(p<0.001)$.

This chapter supports the use of serial IVUS imaging for the assessment of plaque progression-regression as a surrogate endpoint of cardiovascular event risk. We found a link between the estimated cardiovascular risk, actual cardiovascular events and the rate of plaque progression.

Chapter 6 assesses with serial IVUS the remodeling behaviour (changes in total vessel area) of atherosclerotic left main plaques. The changes in lumen area correlated strongly with changes in vessel $(p<0.0001)$, but not with changes in plaque+media area. Lumen reduction resulted from vessel reduction (sometimes despite plaque decrease), plaque increase (with or without vessel increase), or both. More than $30 \%$ of lesions show a negative remodeling behaviour. There was no relation between negative remodeling versus IVUS plaque composition, initial plaque burden, and other patients characteristics (demographics, medication, and laboratory data).

This chapter provides with serial IVUS evidence of coronary remodeling and reveals a broad spectrum of remodeling directions in atherosclerotic left main plaques. The variability of the remodeling responses may partially explain progressive lumen narrowing in some individuals despite of a lack of plaque progression.

The non-serial IVUS assessment of coronary remodeling (remodeling index), which is only a substitute of true remodeling (serial changes in total vessel area), is validated in Chapter 7. Overall, the follow-up remodeling index correlated directly with changes in lesion site total vessel area (baseline-to-follow-up). In nearly $90 \%$ of lesions with a follow-up remodeling index greater than 1 (non-serial positive remodeling), there was a previously documented increase in total vessel area (serial positive remodeling).

This chapter states that the remodeling index serves as an acceptable substitute for true (serial) coronary remodeling.

Glagov's histopathological observation and non-serial IVUS studies concluded that compensatory coronary remodeling diminishes as $40 \%$ atherosclerotic plaque burden is reached. In Chapter 8 we test this hypothesis with serial IVUS examinations in 46 atherosclerotic non-stenotic left main stems. Overall, there was no relation between baseline plaque burden versus subsequent changes in vessel area. The frequency of positive serial remodeling (vessel area increase) versus 
negative or intermediate serial remodeling (no change or decrease) were similar in both, plaques with a plaque burden smaller and greater than $40 \%$.

This chapter shows that the baseline plaque burden does not predict the subsequent serial remodeling behaviour as assessed with serial IVUS.

In Chapter 9 we assess the problem of using two different IVUS devices in serial studies. A total of 33 human coronary plaques were studied in vitro with two different IVUS systems. We repeatedly measured the total vessel, lumen, and plaque area and plaque burden. Between the "raw" measurements made by the two devices, there was a significant difference for both plaque area and plaque burden. Measurements were then corrected by use of recently introduced calibration formulas; as a result, the differences decreased significantly for all IVUS parameters measured. After correction, the remaining differences between the corrected mechanical and electronical IVUS measurements were similar to differences between repeated measurements with the same IVUS device (i.e., the intraobserver variability).

This chapter shows that the use of different IVUS devices in serial studies may introduce a substantial error as a result of system-related differences and that the application of dedicated calibration formulas allows to correct for these differences by decreasing them to the level of intraobserver variability.

Volumetric radiofrequency-based (RF) IVUS data may be an interesting endpoint of IVUS studies that evaluate the effect of anti-atherosclerotic pharmacological therapies not only on plaque geometrics but also on plaque composition. In Chapter 10 we assess in mild-to-moderate atherosclerotic human coronary segments the reproducibility of volumetric RF-IVUS in vivo by comparing analyses from the same pullback, repeated pullbacks, and by different observers. We found that the reproducibility of volumetric compositional RF-IVUS measurements from the same pullback is relatively high, but lower than the reproducibility of geometrical IVUS measurements. Measurements from repeated pullbacks and by different observers showed acceptable reproducibilities; the volumetric measurement of the necrotic-core showed on average the highest reproducibility of the compositional RF-IVUS measurements.

This chapter demonstrates an acceptable reproducibility of volumetric RF-IVUS measurements for the use in IVUS studies with a serial study design.

Chapter 11 gives an up-date on the current knowledge gained from serial observational and randomized IVUS studies of coronary atherosclerosis. Topics are (1) cholesterol, lipid-lowering therapy, and progression-regression of coronary 
atherosclerosis as assessed with serial IVUS, (2) the value of IVUS assessed plaque progression-regression as a surrogate marker of cardiovascular event risk, (3) serial assessment of plaque composition with RF-IVUS, (4) assessment of coronary vessel remodeling in serial IVUS trials, and (5) new perspectives of serial imaging of coronary atherosclerosis. 



\section{Samenvatting en Conclusies}





\section{Samenvatting en Conclusies}

Cardiovasculaire ziekte is een belangrijke oorzaak van morbiditeit en mortaliteit in landen met een westerse levensstijl. Betrouwbare kwantitatieve bepaling van de omvang van de coronair atherosclerose is van belang om zowel de progressie van de ziekte alsmede het effect van anti-atherosclerotische therapie te kunnen beoordelen.

Coronaire angiografie is een techniek waarbij een silhouet van het lumen zichtbaar wordt gemaakt. De vaatwand waar het atherosclerotisch ziekteproces voornamelijk is gelokaliseerd wordt daarbij niet in beeld gebracht. Met behulp van intravasculair ultrageluid (IVUS) is het echter mogelijk transmurale tomografische beelden van de kransslagaders te verkrijgen waarop direct metingen mogelijk zijn om lumen, plaque en vaatdimensies te kwantificeren.

Seriële IVUS metingen maken het mogelijk om de mate van de coronair plaque progressie of regressie en veranderingen in vaatdimensies (remodelleringsproces) te kwantificeren. Dit wordt geïntroduceerd in Hoofdstuk 1. Hoofdstukken 2-4 van dit proefschrift zijn gericht op de relatie tussen serumparameters en progressieregressie van atherosclerose in de linker hoofdstam. Hieropvolgend wordt in Hoofdstuk 5 de relatie tussen het risico op cardiovasculaire gebeurtenissen en met IVUS gemeten progressie-regressie van de plaque beschreven. Hoofdstukken 6-8 tonen het remodelleringsgedrag van coronaire laesies in een serieel IVUS design. Dit wordt getoond middels (1) aanwezigheid van seriële remodellering (Hoofdstuk 6), (2) de validatie van een niet-seriële bepaling van coronaire remodellering (Hoofdstuk 7) en (3) de validatie van de Glagov's histopathologische remodelleringshypothese (Hoofdstuk 8). In Hoofdstuk 9 worden verschillen in metingen tussen verschillende IVUS systemen in vitro en het gebruik van een gevalideerde gespecialiseerde kalibratieformule voor correctie beschreven. In Hoofdstuk 10 wordt gekeken naar volumetrische op radiofrequentie-gebaseerde IVUS analyses van atherosclerotische plaque compositie. Tevens wordt de reproduceerbaarheid van deze techniek voor gebruik in seriële studies geëvalueerd. Ten slotte wordt in Hoofdstuk 11 een overzicht gegeven over de huidige kennis over coronaire atherosclerose, die verkregen is middels seriële IVUS analyses in observationele en gerandomiseerde farmacologische interventie-trials.

In Hoofdstuk 2 wordt de progressie danwel regressie van atherosclerotische plaques in de linker hoofdstam in relatie tot serumcholesterol geëvalueerd. De resultaten zijn gebaseerd op IVUS data van 60 coronairen. De periode tussen eerste en tweede IVUS onderzoek was $18 \pm 9$ maanden. Er was (1) een positieve 
lineaire relatie tussen low-density lipoprotein (LDL) cholesterol en veranderingen in plaque oppervlakte $(r=0.41, p<0.0001)$ met $(2)$ een $L D L$ cholesterol waarde van $75 \mathrm{mg} / \mathrm{dL}$ als de cutoff waarde. Regressie-analyse liet zien dat bij deze waarde gemiddeld geen toename van plaque kon worden voorspeld; (3) een negatief lineaire relatie tussen high-density lipoprotein cholesterol en veranderingen in het oppervlakte van de plaque; en (4) een negatief lineaire relatie tussen LDL cholesterol en de veranderingen in de grootte van lumen.

Dit hoofdstuk vergroot onze kennis over de klinische relatie tussen serumlipiden en het risico op cardiovasculaire gebeurtenissen door het aantonen van de relatie tussen serumcholesterolwaardes en seriële veranderingen in dimensies van de coronaire plaque. Daarmee benadrukt dit hoofdstuk mede het belang van een aggressief lipiden-verlagende beleid bij patiënten met coronaire vaatziekten.

In Hoofdstuk 3 wordt de relatie tussen serum cholesterol, leeftijd en plaque progressie, zoals bepaald met seriële IVUS in de hoofdstam, bekeken. Op basis van leeftijd werden 60 patiënten verdeeld in tertielen: tertiel 1 had een gemiddelde leeftijd van 48 jaar (33 tot 55 jaar), tertiel 2 had een gemiddelde leeftijd van 58 jaar (55 tot 61 jaar) en tertiel 3 had een gemiddelde leeftijd van 66 jaar (61 tot 83 jaar). Er werd een positieve lineaire relatie gevonden tussen LDL cholesterol en veranderingen in de oppervlakte van de plaque in alle tertielen. In tertiel 2 en 3 was deze verandering statistisch significant $(p<0.02)$, in tertiel 1 was er een sterke trend. Door middel van regressie-analyse werd bepaald dat $80 \mathrm{mg} / \mathrm{dl}$ in alle tertielen de waarde is, waarbij LDL cholesterol niet langer correspondeert met toename van plaque oppervlakte.

Dit hoofdstuk suggereert een potentieel klinisch voordeel van (agressieve) lipideverlagende therapie in oudere patiënten. Deze suggestie wordt gesteund door de aangetoonde relatie tussen $L D L$ cholesterol en plaqueprogressie gedurende het ouder worden.

In Hoofdstuk 4 wordt de relatie tussen de cardiovasculaire risicofactoren lipoproteïne (a) $[L p(a)]$ en fibrinogeen vergeleken met coronaire plaque progressie en cardiovasculaire gebeurtenissen. Er werd een positieve correlatie gevonden tussen $L p(a)$, fibrinogeen en veranderingen in plaque oppervlakte. Patiënten met toename van plaque hadden hogere $L p(a)$ en fibrinogeen waarden dan patiënten met afname van de plaque. $L p(a)$ was onafhankelijk geassocieerd met toename van plaque $(p<0.001)$, deze associatie werd niet gezien bij fibrinogeen. Patiënten met cardiovasculaire gebeurtenissen hadden hogere $L p(a)$ en fibrinogeen waarden $(p<0.0001)$. Deze markers zijn beide onafhankelijk geassocieerd met cardiovasculaire gebeurtenissen. 
Dit hoofdstuk demonstreert een relatie tussen klassieke en nieuwe cardiovasculaire risicofactoren, atherosclerotische plaqueprogressie en cardiovasculaire gebeurtenissen.

In Hoofdstuk 5 wordt het (1) geschat cardiovasculair risico (gecombineerd uit 3 bekende risicoscores voor primaire preventie (PROCAM, SCORE en Framingham) en (2) het voorkomen van cardiovasculaire gebeurtenissen vergeleken met toename van plaque, bepaald met seriële IVUS in atherosclerotische hoofdstammen. In patiënten met het hoogste geschatte risico op cardiovasculaire gebeurtenissen werd meer plaque progressie gemeten dan bij patiënten met een lager risico. Er werd een positieve lineaire relatie gevonden tussen het risico op klinische gebeurtenissen en plaque progressie. Gedurende follow-up kregen 18 patiënten een cardiovasculaire gebeurtenis. Bij deze patiënten was er een significant grotere toename van plaque dan bij patiënten zonder events $(p<0.001)$.

Dit hoofdstuk ondersteunt het gebruik van serieel IVUS onderzoek voor de bepaling van plaque progressie of regressie als een surrogaat eindpunt voor risico op een cardiovasculaire gebeurtenis. Er werd een verband gevonden tussen geschat cardiovasculair risico, daadwerkelijk opgetreden cardiovasculaire gebeurtenissen en de mate van plaque progressie.

In Hoofdstuk 6 wordt door middel van seriële IVUS onderzoeken het remodelleringsgedrag (verandering in totaal vaatoppervlak) van atherosclerotische plaques in de hoofdstam bepaald. Veranderingen in oppervlakte van het lumen correleren sterk met veranderingen in de oppervlakte van het vat $(p<0.0001)$, maar niet met veranderingen in de oppervlakte van de plaque. Afname van het lumenoppervlakte kon het gevolg zijn van afname van oppervlakte van het vat (soms ondanks afname van plaque), toename van de plaque (met of zonder toename van vaatoppervlak) of beide. Bij meer dan $30 \%$ van de laesies werd negatief remodelleringsgedrag geobjectiveerd. Er werd geen relatie gevonden tussen negatieve remodellering, IVUS plaque compositie, initiële plaque burden en andere patiëntkarakteristieken (demografie, medicatie, laboratoriumonderzoek).

Dit hoofdstuk toont via seriële IVUS bepalingen dat er aanwijzingen zijn voor coronaire remodellering hetgeen een breed spectrum van remodelleringseigenschappen omvat in de atherosclerotische plaques in de hoofdstam. Variabiliteit van remodellering zou bij specifieke patiënten kunnen verklaren waarom het lumenoppervlakte progressief afneemt ondanks effectieve modificatie van het lipidenprofiel of andere risicofactoren. 
De validatie van de niet-seriële IVUS bepalingen van coronaire remodellering (remodelling index), welke alleen een substituut is van echte remodellering (seriële veranderingen in totaal vaatoppervlak) wordt beschreven in Hoofdstuk 7. De remodelling index correleerde direct met veranderingen in totaal vaatoppervlak op de plek van de laesie (over een periode van baseline tot follow-up). In bijna 90\% van de laesies met een follow-up remodelling index groter dan 1 (niet-seriële positieve remodellering), was eerder een toename in totaal vaatoppervlak gedocumenteerd (seriële positieve remodellering).

In dit hoofdstuk wordt aangetoond dat de remodelling index als een acceptabel substituut voor ware (seriële) coronaire remodellering kan dienen.

Glagov's histopathologische observatie en niet-seriële IVUS studies hebben aangetoond dat compensatoire coronaire remodellering verminderd als er een $40 \%$ atherosclerotische "plaque burden" wordt bereikt. In Hoofdstuk 8 testen wij deze hypothese middels seriële IVUS onderzoeken in 46 atherosclerotische nietstenotische hoofdstammen. Globaal was er geen relatie te ontdekken tussen baseline "plaque burden" en verdere verandering in vaatoppervlak. De frequentie van positieve seriële remodellering (toename in vaatoppervlakte) versus negatieve of intermediaire seriële remodellering (afname of geen verandering) waren gelijk in zowel plaques met een "plaque burden" kleiner of groter dan $40 \%$.

Dit hoofdstuk toont dat baseline "plaque burden" geen opvolgend serieel remodelleringsgedrag kan voorspellen, zoals bekeken met seriële IVUS studies.

In Hoofdstuk 9 beoordelen wij het probleem wat op kan treden als 2 verschillende IVUS systemen worden gebruikt in seriële studies. Een totaal van 33 menselijke coronaire plaques werd in vitro bestudeerd met twee verschillende IVUS apparaten. Bij herhaling werd het totale vat, lumen, plaque-oppervlakte en "plaque burden" gemeten. Tussen de "ruwe" metingen gemaakt door de 2 systemen was er een significant verschil in zowel plaque oppervlakte als "plaque burden". Metingen werden hierna gecorrigeerd met behulp van recent geïntroduceerde kalibratieformules; dit leidde tot significant kleinere verschillen tussen beide IVUS systemen. Na correctie waren de resterende verschillen tussen de gecorrigeerde mechanische en electronische IVUS metingen gelijk aan de veranderingen die gezien werden bij herhaalde metingen met een en hetzelfde IVUS systeem (intraobserver variabiliteit).

Dit hoofdstuk toont aan dat het gebruik van verschillende IVUS apparatuur in seriële studies kan leiden tot belangrijke fouten. Dit is het resultaat van systeemgerelateerde verschillen. Applicatie van een specifieke kalibratieformule corrigeert deze verschillen en verlaagt ze tot het niveau van de intra-observer 
variabiliteit.

Volumetrische op radiofrequentie gebaseerde (RF) IVUS metingen kunnen een belangrijke onderzoeks modaliteit vormen in studies welke het effect van antiatherosclerotische farmacologische therapie op plaque geometrie en samenstelling evalueren. In Hoofdstuk 10 beoordelen wij, in vivo, de reproduceerbaarheid van volumetrische RF-IVUS metingen in menselijke coronaire segmenten met milde tot matige atherosclerose door het vergelijken van analyses van dezelfde pullback, herhaalde pullbacks en veschillende onderzoekers. Wij zagen dat de reproduceerbaarheid van volumetrische compositionele RF-IVUS metingen van dezelfde pullback relatief hoog is, maar lager dan de reproduceerbaarheid van geometrische IVUS metingen. Metingen van herhaalde pullbacks en metingen door verschillende onderzoeker toonden acceptabele reproduceerbaarheden; de volumetrische berekening van de "necrotic core" toonde gemiddeld de hoogste reproduceerbaarheid van de compositionele RF-IVUS gebaseerde bepalingen.

Dit hoofdstuk demonstreert een acceptabele reproduceerbaarheid van volumetrisch op RF-IVUS gebaseerde bepalingen van plaque compositie voor het gebruik in IVUS studies met een serieel studie design.

Hoofdstuk 11 geeft een update van de huidige kennis verkregen door seriële observationele en gerandomiseerde IVUS studies van coronaire atherosclerose. Onderwerpen zijn (1) cholesterol, lipideverlagende therapie en progressieregressie van coronaire atherosclerose zoals bestudeerd met seriële IVUS, (2) de waarde van door middel van IVUS gemeten progressie-regressie van plaque als surrogaat marker voor het risico op cardiovasculaire gebeurtenissen, (3) seriële meting van plaque compositie met RF-IVUS, (4) beoordeling van remodellering van coronaire vaten in seriële IVUS trials en (5) nieuwe inzichten in seriële beeldvormingsmethoden voor coronaire atherosclerose. 

Acknowledgements 



\section{Acknowledgements}

I am very grateful to Prof. Clemens von Birgelen for being my promotor and mentor in cardiology research throughout such a long period of time. Dear Clemens, you initiated and fully supported my activities at University Duisburg-Essen in Germany, and thereafter at Medisch Spectrum Twente in Enschede. It was and still is a great pleasure to learn from your experience, as you are one of the leading experts in the field of invasive coronary imaging. After such a long scientific journey and intense cooperation, I am proud to call you a real friend of mine.

It was a honour and privilege to work with Prof. Gary Mintz from Cardiovascular Research Foundation in New York, one of the most excellent experts of clinical research in interventional cardiology and intravascular ultrasound. Dear Gary, I am very grateful for the many years of cooperation which gave me the opportunity to learn so much from you.

I am also grateful to Prof. Raimund Erbel, the Director of the West-German Heart Center of the University Duisburg-Essen. His inspiring ideas and the continuous support by one of the pioneers of cardiovascular ultrasound and interventional cardiology were very stimulating for the entire research project.

It was a pleasure to work with Dr. Patrick Verhorst, who is currently chief instructor of my clinical training at Medisch Spectrum Twente in Enschede. Patrick, I would like to thank you for your belief in me and your enthusiastic motivation and support of my clinical and research activities. It is really great to be trained by you and your team in Enschede as you create a wonderful stimulating atmosphere of teaching and learning.

Dr. Job van der Palen supported my research by giving expert statistical advice. Job, I am very grateful for your statistical assistance. It is almost unbelievable how you were able to convert apparently difficult challenges into simple questions which you were able to promptly answer.

I would like to thank the entire team of the cardiac catheterization laboratory in Essen for their support of our research. I would like to express my particular gratitude to Dr. Dirk Böse (thank you for your kind introduction to intravascular ultrasound imaging), Dr. Heinrich Wieneke, Dr. Mario Gössl, Dr. Holger Eggebrecht, Prof. Axel Schmermund, Prof. Michael Haude, and Prof. Dietrich Baumgart. I would also like to thank Dr. Olaf Dirsch from the Institute of Pathology of the University of Duisburg-Essen, who facilitated our in vitro studies. 
I am also grateful to the cardiologists, staff of the cardiac catheterization laboratory, cardiovascular research personnel, technicians, nurses, and other co-workers of the Department of Cardiology at Medisch Spectrum Twente, who supported our research. In particular, I am very grateful to the interventional cardiologists Gert van Houwelingen, Martin ("Mr. Adenosine") Stoel, Hans Louwerenburg, and Dr. Frits de Man for performing intravascular ultrasound examinations of such a high quality.

I truly appreciated the help and motivation of all cardiologists and cardiology fellows in Enschede and the support by the secretaries Wendy Grooters, Nicole de Jong (Medisch Spectrum Twente), and Sylvia Winters (University Twente). I apologize for being unable to mention all people who supported me and our research project. I am grateful to all of them.

My "paranimfs" Karin Kraaier and Reinhart Dorman supported me very well during the process of this promotion. Karin and Reinhart, I would like to express my deep gratitude for being so helpful.

I was (initially) very grateful to Anke van de Water for giving me her bicycle which improved my mobility in Enschede. Soon after, the bike temporarily increased the mobility of my right elbow as a result of a fall from that bike. I very much appreciate the excellent skillful work of Dr. Remko Karthaus - my orthopedic surgeon at Medisch Spectrum Twente, who fixed that multi-fractured elbow. Now that I am fully recovered, I think life is much easier this way ... than potentially becoming the first one-armed interventional cardiologist in the Netherlands. By the way, my cofellow Karin Kraaier who took the bike over is meanwhile fully recovered from a fracture of her right hand ... the result of a fall from the very same bike. The good news is that in the meantime the "fatal bike" has been stolen, which lowered the risk of injured cardiology fellows significantly! ... Did anybody hear about a thief who fell off a bicycle?

I would like to thank my dear parents for confidence, motivation, and emotional support.

Last but not least, I thank you, Nadine, for your love, support, and encouragement throughout the years and during my move to Enschede. You showed great understanding for my eagerness in research and the many evenings and weekends that I spent on data analysis and writing. But above all, I would like to thank you for being such a great mother of our sweet little daughter Leni. 


\section{Curriculum Vitae}





\section{Curriculum Vitae}

Marc Hartmann was born on February 3, 1978, in Duisburg, Germany. In 1997 he qualified for University at the Sophie-Scholl-Gymnasium in Oberhausen, Germany. He completed medical and clinical training at the Medical University of DuisburgEssen in Germany in 2004. Since 2001, as a student, he worked as a member of the Intravascular Ultrasound Research Group, chaired by Prof. von Birgelen at the University Duisburg-Essen and thereafter at Medisch Spectrum Twente in Enschede, the Netherlands. Since 2005, he is being trained at Medisch Spectrum Twente as a cardiologist. Marc and his wife Nadine have a little daughter called Leni who was born in 2007. 



\section{Publications}





\section{Publications}

[1] Hartmann M, Mattern ESK, Huisman J, van Houwelingen GK, de Man FHAF, Stoel MG, Danse PW, Louwerenburg HW, von Birgelen C Reproducibility of volumetric intravascular ultrasound radiofrequency-based analysis of coronary plaque composition in vivo The International Journal of Cardiovascular Imaging 2008; - in press

[2] Kraaier K, Hartmann M, Stoel MG, von Birgelen C Intermittent spastic coronary occlusion at site of non-significant atherosclerotic lesion requiring stent implantation Netherlands Heart Journal 2008; - in press

[3] Hartmann M, van Es J, Galjee MA, van der Burgh PH, de Bruin WI, Said SA, von Birgelen $\mathrm{C}$

Cardiac imaging in a symptomatic patient with multiple coronary artery-left ventricular microfistulae

Heart and Vessels 2007;22:428-431.

[4] Hartmann M, Verhorst PMJ, von Birgelen C Isolated „superdominant“ single coronary artery: a particularly rare coronary anomaly

Heart 2007;93:687.

[5] Aliyary S, Mariani MA, Verhorst PM, Hartmann M, Stoel MG, von Birgelen C Staged therapeutic approach in spontaneous coronary dissection The Annals of Thoracic Surgery 2007;83:1879-1881.

[6] Hartmann M, von Birgelen C, Mintz GS, van Houwelingen GK, Eggebrecht $H$, Böse D, Wieneke H, Verhorst PMJ, Erbel R

Relationship between plaque progression and low-density lipoprotein cholesterol during aging as assessed with serial long-term ( $\geq 12$ months) followup intravascular ultrasound of the left main coronary artery

The American Journal of Cardiology 2006;98:1419-1423.

[7] von Birgelen C, Hartmann M

Atherosclerotic coronary plaque burden and cardiovascular risk factors: singlepoint versus serial intravascular ultrasound assessment Journal of the American College of Cardiology 2006;48:1914-1915. 
[8] von Birgelen C, Hartmann M

High-dose statins and atherosclerosis regression

The Journal of the American Medical Association 2006;296(15):1837.

[9] Hartmann M, von Birgelen C, Mintz GS, Stoel MG, Eggebrecht H, Wieneke H, Fahy M, Neumann T, van der Palen J, Louwerenburg HW, Verhorst PMJ, Erbel $\mathrm{R}$

Relation between lipoprotein(a) and fibrinogen and serial intravascular ultrasound plaque progression in left main coronary arteries Journal of the American College of Cardiology 2006;48;446-452.

[10] Metz E, Hartmann M, von Birgelen C, Haleboos MP, Verhorst PMJ Major dehiscence of infected aortic valve prosthesis with "rocking motion" but without diastolic paravalvular regurgitation

The International Journal of Cardiovascular Imaging 2006;22:771-774.

[11] Hartmann M, von Birgelen C, Mintz GS, Verhorst PMJ, Erbel R Relation between baseline plaqueburden and subsequent remodeling of atherosclerotic left main coronary arteries: a serial intravascular ultrasound study with long-term ( $\geq 12$ months) follow-up European Heart Journal 2006;27:1778-1784.

[12] Hartmann M, von Birgelen C, Mintz GS, Deppermann N, Dirsch O, Stoel MG, van Houwelingen GK, Louwerenburg HW, Verhorst PMJ, Erbel R Dedicated calibration formulas permit correction of differences between measurements by different IVUS devices as demonstrated in atherosclerotic human coronary arteries in vitro The International Journal of Cardiovascular Imaging 2006;22:605-613.

[13] von Birgelen C, Hartmann M, Mintz GS, Böse D, Eggebrecht H, Neumann T, Gössl M, Wieneke H, Schmermund A, Stoel MG, Verhorst PM, Erbel R Remodeling index compared to actual vascular remodeling in atherosclerotic left main coronary arteries as assessed with long-term ( $\geq 12$ months) serial intravascular ultrasound Journal of the American College of Cardiology 2006;47:1363-1368.

[14] von Birgelen C, Hartmann M Intravascular ultrasound assessment of coronary atherosclerosis and percutaneous interventions Minerva Cardioangiologica 2004;52:391-406. 
[15] von Birgelen C, Hartmann M, Mintz GS, van Houwelingen KG, Deppermann $N$, Schmermund A, Böse D, Eggebrecht H, Neumann T, Gössl M, Wieneke H, Erbel R

Relationship between cardiovascular risk as predicted by established risk scores versus plaque progression as measured by serial intravascular ultrasound

Circulation 2004;110:1579-1585.

[16] von Birgelen C, Hartmann M, Mintz GS, Böse D, Eggebrecht H, Gössl M, Neumann T, Baumgart D, Wieneke H, Schmermund A, Haude M, Erbel R Spectrum of remodeling behavior observed with serial long-term ( $\geq 12$ months) follow-up intravascular ultrasound studies in left main coronary arteries The American Journal of Cardiology 2004;93:1107-1113.

[17] von Birgelen C, Hartmann M, Mintz GS, Baumgart D, Schmermund A, Erbel R Relation between progression and regression of atherosclerotic left main coronary artery disease and serum cholesterol levels as assessed with serial long-term ( $\geq 12$ months) follow-up intravascular ultrasound Circulation 2003;108:2757-2762. 



\section{STELLINGEN}

\section{behorende bij het proefschrift \\ Serial Intravascular Ultrasound Assessment of Coronary Atherosclerosis Progression and Remodeling}

1. Intravascular ultrasound (IVUS) is een nauwkeurige methode met een goede reproduceerbaarheid om het beloop van de coronaire atherosclerose te vervolgen.

2. Er is een significante relatie tussen het LDL-cholesterol gehalte en de mate van progressie van coronaire atherosclerose, gequantificeerd met serieel IVUS onderzoek. (Dit proefschrift)

3. Het beleid van aggressieve verlaging van het LDL-cholesterol gehalte kan de progressie van coronaire atherosclerose stoppen en soms regressie van plaques induceren.

4. $\quad E r$ is een verband tussen het geschatte cardiovasculaire risico, het daadwerkelijk optreden van cardiovasculaire events en de mate van coronaire atherosclerose progressie, bepaald met serieel IVUS onderzoek. (Dit proefschrift)

5. Zelfs het effect van "Bratwurst \& Sauerkraut" op progressie van coronaire atherosclerose zou met serieel IVUS onderzoek getest kunnen worden om de primaire preventie van hart- en vaatziekten te optimaliseren.

6. Serieel IVUS onderzoek als surrogat eindpunt in farmacologische interventie trials zou het ontwikkelingsproces van nieuwe antiatherosclerotische therapieën kunnen bespoedigen.

7. "Just when I thought I was out ... they pull me back in !" omschrijven de belevingen van IVUS catheters tijdens serieel IVUS onderzoek.

(Michael Corleone in de film Godfather III)

8. Serieel IVUS toont een hoge variabiliteit van coronaire remodeling hetgeen bij specifieke patienten zou kunnen verklaren waarom de coronaire lumendimensie afneemt ondanks effectief modificeren van het lipidenprofiel en andere risicofactoren. (Dit proefschrift)

9. De remodeling index is een acceptabel substituut voor de ware (seriële) coronaire remodeling in een onderzoekspopulatie. (Dit proefschrift)

10. Wetenschap gaat vooruit, als observaties ons dwingen veronderstellingen aan te passen. (vrij geformuleerd naar Vera Rubin) 